\title{
DISCRETE SERIES FOR SEMISIMPLE LIE GROUPS. II EXPLICIT DETERMINATION OF THE CHARACTERS \\ BY
}

\author{
HARISH-CHANDRA
}

The Institute for Advanced Study, Princeton, N.J., U.S.A.

\section{Table of Contents}

§ 1. Introduction. . . . . . . . . . . . . . . . . . . . . . 2

Part I. analysis in the space $\mathrm{C}(G)$

§ 2. Representations on a locally convex space. . . . . . . . . . . . . . . . . . 5

§ 3. Absolute convergence of the Fourier series. . . . . . . . . . . . . . . . 8

§4. Proof of Lemma 7 . . . . . . . . . . . . . . . . . . . . . . 11

§. Differentiable vectors and Fourier series in function spaces . . . . . . . . . 12

§6. Proof of Lemma 4 . . . . . . . . . . . . . . . . . . . . . . . 14

$\S 7$. Some elementary facts about $\sigma$ and $\Xi$. . . . . . . . . . . . . 15

§8. Proof of Theorem 1. . . . . . . . . . . . . . . . . . . 18

\$9. The space $C(G)$. . . . . . . . . . . . . . . . . . . . . . . . . . . 19

\$ 10. The left- and right-regular representations on $C(G)$. . . . . . . . . . . . 20

§11. Spherical functions . . . . . . . . . . . . . . . . . . . 23

\$12. Application to $\mathrm{C}_{F}(G)$. . . . . . . . . . . . . . . . . . . . . 25

§ 13. Density of $C_{c}{ }^{\infty}(G)$ in $\mathrm{C}(G)$. . . . . . . . . . . . . . . . . . . . 26

\$14. An inequality . . . . . . . . . . . . . . . . . . . . . . . . 27

\$ 15. The mapping of $\mathrm{C}(G)$ into $\mathrm{C}(\tilde{M})$. . . . . . . . . . . . . . . . . . . . . . 29

§16. Proof of Theorem 4. . . . . . . . . . . . . . . . . . . . 30

$\S 17$. Convergence of certain integrals . . . . . . . . . . . . . . . . . . . 32

\$ 18. The mapping $f \rightarrow F_{f}$. . . . . . . . . . . . . . . . . . . . . . . . . 35

$\S 19$. A criterion for an invariant eigendistribution to be tempered . . . . . . . . 38

\$20. Proof of Theorem 8 . . . . . . . . . . . . . . . . . . . . . . 45

\$21. Proof of an earlier conjecture . . . . . . . . . . . . . . . . . 46

\$22. Proof of Lemma 40 (first part). . . . . . . . . . . . . . . . . . . . . . 48

1-662900. Acta mathematica. 116. Imprimé le 10 juin 1966. 
§ 23. Proof of Lemma 40 (second part). . . . . . . . . . . . . . . . . . . 49

\$24. Proof of Theorem 9. . . . . . . . . . . . . . . . . . . . . 51

§ 25. Application to tempered representations . . . . . . . . . . . . . . 52

Part II. Spherical FUnCtions and Differential EqUations

§ 26. Two key lemmas and their first reduction . . . . . . . . . . . . . . . . . 54

$\S 27$. The differential equation for $\Phi$. . . . . . . . . . . . . . . . . . . . . 61

$\S 28$. Some estimates for $\Phi$ and $\Psi_{\zeta}$. . . . . . . . . . . . . . . . . . . . . . . . 63

$\S$ 29. The function $\Theta$. . . . . . . . . . . . . . . . . . . . . . . 64

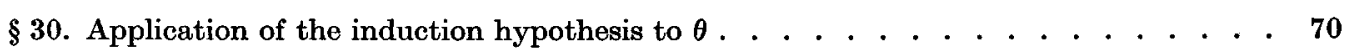

\$31. Completion of the proofs of Lemmas 42 and 43 . . . . . . . . . . . . . . . . 74

Part III. Applications to harmonic analysis on $G$

\$32. Lemma 64 and its consequences . . . . . . . . . . . . . . . . . . . . . 75

§33. Proof of a conjecture of Selberg . . . . . . . . . . . . . . . . . 77

\$34. The behaviour of certain eigenfunctions at infinity . . . . . . . . . . . . . 78

§35. Eigenfunctions of 8 in $\mathrm{C}(G)$. . . . . . . . . . . . . . . . 81

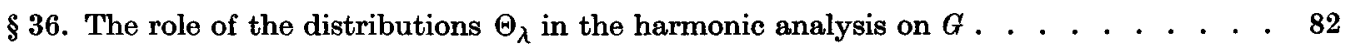

\$37. The discrete series for $G$. . . . . . . . . . . . . . . . . . . . . . . . . 87

§ 38. Proof of Lemma 65 . . . . . . . . . . . . . . . . . . . . . . . . 89

\$39. The existence of the discrete series . . . . . . . . . . . . . . . . . . 90

$\S 40$. The characters of the discrete series . . . . . . . . . . . . . . . 90

\$4. Explicit determination of these characters. . . . . . . . . . . . . . . 96

PART IV. SOME INEqUalities AND THEIR CONSEqUences

$\$ 42$. Proof of the inequalities . . . . . . . . . . . . . . . . . 100

$\S 43$. Applications of the above inequalities . . . . . . . . . . . . . 105

$\S 44$. Proof of Lemma 21. . . . . . . . . . . . . . . . . . . . 109

$\S 45$. Appendix . . . . . . . . . . . . . . . . . . . . 109

References. . . . . . . . . . . . . . . . . . . . . . 110

\section{§ 1. Introduction}

Let $G$ be a connected semisimple Lie group and $K$ a maximal compact subgroup of $G$. We shall show in this paper that $G$ has a discrete series (see [ $4(d), \S 5]$ ) if and only if it has a compact Cartan subgroup $B$. Let $\mathcal{E}_{d}$ denote the set of all equivalence classes of irreducible unitary representations of $G$, which are square-integrable. For any $\omega \in \mathcal{E}_{d}$, let $\Theta_{\omega}$ denote the character, $\chi_{\omega}$ the infinitesimal character and $d(\omega)$ the formal degree (see $[4(\mathrm{~d}), \S 3]$ ) of $\omega$. Then it is known [4 (d), $\S 5]$ that the distribution 


$$
T=\sum_{\omega \in \varepsilon_{d}} d(\omega) \Theta_{\omega}
$$

represents the contribution of the discrete series to the Plancherel formula of $G$. We intend to obtain explicit formulas for $d(\omega), \Theta_{\omega}$ and $T$.

Let $g$ and $\mathfrak{b}$ be the Lie algebras of $G$ and $B$ respectively. Then the character group of $B$ may be identified with a lattice $L$ in the space of all real-valued linear functions $\mathfrak{F}$ on $(-1)^{\frac{1}{2}}$ b. Put

$$
\varpi(\lambda)=\prod_{\alpha>0}\langle\lambda, \alpha\rangle \quad(\lambda \in \mathfrak{F})
$$

where $\alpha$ runs over all positive roots of $(\mathfrak{g}, \mathfrak{b})$ and the scalar product is defined, as usual, by means of the Killing form. Let $L^{\prime}$ denote the set of all $\lambda \in L$ where $\varpi(\lambda) \neq 0$. Then, for every $\lambda \in L^{\prime}$, we have constructed in $[4,(p)]$, an invariant eigendistribution $\Theta_{\lambda}$ of 8 on $G$. Put $q=\frac{1}{2} \operatorname{dim} G / K$ and $\varepsilon(\lambda)=\operatorname{sign} \varpi(\lambda)\left(\lambda \in L^{\prime}\right)$. Our main result (Theorem 16) states that the distributions $(-1)^{a} \varepsilon(\lambda) \Theta_{\lambda}\left(\lambda \in L^{\prime}\right)$ are exactly the characters of the discrete series and

$$
T=c^{-1}(-1)^{q} \sum_{\lambda \in L^{*}} \varpi(\lambda) \Theta_{\lambda}
$$

where $c$ is a positive constant.

Let $W$ be the Weyl group of $(\mathfrak{g}, \mathfrak{b})$ and $\tilde{B}$ the normalizer of $B$ in $G$. Then $W_{G}=\tilde{B} / B$ may be regarded as a subgroup of $W$. Define $\chi_{\lambda}\left(\lambda \in L^{\prime}\right)$ as usual (see $[4(\mathrm{p}), \S 29]$ ) and let $\mathcal{E}_{d}(\lambda)$ denote the set of all $\omega \in \mathcal{E}_{d}$ such that $\chi_{\omega}=\chi_{\lambda}$. Then (1)

$$
\left[\mathcal{E}_{d}(\lambda)=[W(\lambda)]\left[W_{G}\right]^{-1},\right.
$$

where $W(\lambda)$ is the set of all $s \in W$ such that $s \lambda \in L$.

Fix $\lambda \in L^{\prime}$ and let $\Theta_{\lambda, \mathrm{D}}\left(\delta \in \mathcal{E}_{K}\right)$ denote the Fourier components of $\Theta_{\lambda}$ (see [4 (q), $\left.\S 17\right]$ ). Then $\Theta_{\lambda, \delta}$ are analytic functions and it is one of the principal steps of the proof to show that they lie in $L_{2}(G)$ (Theorem 12). This is done by means of Lemma 67, whose proof is based on two key results (Lemmas 42 and 43 ), which are derived from a study of certain differential equations.

This paper is divided into four parts. After recalling some known facts about representations on a locally convex space, we prove Theorem 1, which seems to play an important role in harmonic analysis. Then we introduce the space $C(G)$, which is the analogue of the Schwartz space over $\mathbf{R}^{n}$. Theorem 2 asserts that $C_{c}{ }^{\infty}(G)$ is dense in $\mathcal{C}(G)$ and Theorem 3 allows us to reduce certain problems from $G$ to a proper subgroup. Theorem 4 contains a general result which implies the convergence of certain integrals (Theorems 5 and 6) and thus enables us to define the mapping $f \rightarrow F_{f}(f \in \mathcal{C}(G))$ in $\S 18$. A distribution on $G$ is called tempered if it extends to a continuous linear function on $\mathcal{C}(G)$. Theorem 7 gives a

(1) As usual, $[F]$ denotes the number of elements in a set $F$. 
simple necessary and sufficient condition for an invariant eigendistribution to be tempered. This shows, in particular, that $\Theta_{\lambda}\left(\lambda \in L^{\prime}\right)$ is tempered and Theorem 3 of [4 (q)] remains valid for $f \in \mathcal{C}(G)$ (Theorem 8). This permits us to prove the second conjecture of [4 (k), $\S 16]$ and thus complete the proof of the Plancherel formula for $G / K$. Theorem 9 established a weak estimate for a Fourier component $\Theta_{b}$ of a tempered and invariant eigendistribution $\Theta$. This will be required in Parts II and III.

The main problem in Part II is to determine the behaviour, at infinity, of a $K$-finite eigenfunction $f$ of 8 , which satisfies a weak inequality of the type mentioned above. The principal result (Lemma 43) is that $f$ lies in $L_{2}(G)$ if and only if it lies in $\mathrm{C}(G)$. This is proved by induction on $\operatorname{dim} \mathrm{G}$. By making use of the differential equations, one reduces the problem from $G$ to a proper subgroup $M$ (cf. [4(k)]).

In Part III, we apply the above results to the task of determining the eigenfunctions of 3 in $\mathrm{C}(G)$. Here Lemma 64 plays an important role. It enables us to show that such eigenfunctions do not exist unless $\operatorname{rank} G=\operatorname{rank} K$. As an application we obtain in $\S 33$ a proof of a conjecture of Selberg.

These results are then utilized to determine all the characters of the discrete series, Here the fact that we work in $\mathrm{C}(G)$, rather than $C_{c}{ }^{\infty}(G)$, is decisive. First we show that $\Theta_{\omega}\left(\omega \in \mathcal{E}_{d}\right)$ is tempered and there exists a $\lambda \in L^{\prime}$ such that $\chi_{\omega}=\chi_{\lambda}$. Let $s_{1}, s_{2}, \ldots, s_{r}$ be a complete set of representatives for $W_{G} \backslash W(\lambda)$. Then we prove that

$$
\Theta_{\omega}=\sum_{1 \leqslant i \leqslant r} c_{i} \Theta_{s_{i} \lambda},
$$

where $c_{i}$ are complex numbers. Moreover, by making use of the Schur orthogonality relations, it is possible to establish that

$$
\sum_{1 \leqslant i \leqslant r}\left|c_{i}\right|^{2}=1
$$

On the other hand, one verifies that $c_{i}$ are integers. This proves that

$$
\Theta_{\omega}=(-1)^{a} \varepsilon\left(s_{i} \lambda\right) \Theta_{s_{i} \lambda}
$$

for some $i$ and $d(\omega)=c^{-1}\left[W_{G}\right]|\varpi(\lambda)|$. It should be noticed that the entire discussion of $\S \S 40,41$ is quite similar to Weyl's original treatment of the same problem in the compact case (see $[10, \S 3]$ ). The main task here is to relate the Fourier analysis on $G$, so far as the discrete series is concerned, to that on $B$ (see Theorem 14 and Lemma 81, together with its corollaries). This can be done only by operating in $C(G)$.

Part IV deals with certain inequalities which are needed for the proof of Lemma 21. They will also be useful later, when we come to the continuous series for $G$ in another paper.

Some of the results of this paper have been announced in [4 (1)]. 


\section{Part I. Analysis in the space $\mathcal{C}(G)$}

\section{\$ 2. Representations on a locally convex space}

In this section we recall some elementary and well-known facts about representations on locally convex spaces (see $[2$, p. 109]).

Let $\mathrm{V}$ be a Hausdorff, locally convex (real or complex) vector space and $G$ a locally compact topological group. A representation $\pi$ of $G$ on $V$ is a mapping, which assigns, to every $x \in G$, a continuous endomorphism $\pi(x)$ of $V$ such that the following two conditions hold:

1) $\pi(x y)=\pi(x) \pi(y)(x, y \in G)$ and $\pi(1)=I$.

2) $(x, v) \rightarrow \pi(x) v$ is a continuous mapping of $G \times V$ into $V$.

It is easy to verify that the above two conditions imply the following [2, p. 110].

3) Let $C$ be a compact set in $G$. Then for any neighborhood $U_{0}$ of zero in $V$, we can choose another neighborhood $U$ of zero such that $\pi(C) U \subset U_{0}$.

Let $S$ be the set of all continuous seminorms on $V$. Then the following immediate consequence of 3) will be frequently useful.

4) Given a compact set $C$ in $G$ and an element $v_{0} \in S$, we can choose $v \in S$ such that

for all $x \in C$ and $v \in V$.

$$
v_{0}(\pi(x) v) \leqslant \nu(v)
$$

Conversely we have the following result.

LEMMA 1. Suppose $\pi$ satisfies, in addition to 1), the following two conditions.

$\left.2^{\prime}\right)$ The mapping $x \rightarrow \pi(x) v$ of $G$ into $V$ is continuous for every $v \in V$.

$\left.3^{\prime}\right)$ There exists a neighborhood $U$ of 1 in $G$ with the following property. Given $\nu_{0} \in S$, we can choose $\nu \in S$ such that

for all $u \in U$ and $v \in V$.

$$
v_{0}(\pi(u) v) \leqslant v(v)
$$

Then $\pi$ is a representation of $G$ on $V$.

Fix $x_{0} \in G$ and $v_{0} \in V$. Then

$$
\pi\left(x_{0} u\right) v-\pi\left(x_{0}\right) v_{0}=\pi\left(x_{0}\right) \pi(u)\left(v-v_{0}\right)+\pi\left(x_{0}\right)\left(\pi(u) v_{0}-v_{0}\right)
$$

for $u \in U$ and $v \in V$. Hence it is clear that $\pi\left(x_{0} u\right) v \rightarrow \pi\left(x_{0}\right) v_{0}$ as $u \rightarrow 1$ and $v \rightarrow v_{0}$. This proves our assertion.

Now assume that $V$ is complex and complete, $G$ is unimodular and $d x$ is a Haar measure on $G$. $\pi$ being a representation of $G$ on $V$, we define, for any $f \in C_{c}(G)$, a linear transformation $\pi(f)$ in $V$ by 


$$
\pi(f) v=\int f(x) \pi(x) v d x \quad(v \in V)
$$

It follows from 3) that $\pi(f)$ is continuous. Define the convolution $f * g\left(f, g \in C_{c}(G)\right)$ as usual, so that

Then $\pi(f * g)=\pi(f) \pi(g)$.

$$
(f * g)(x)=\int f(y) g\left(y^{-1} x\right) d y \quad(x \in G)
$$

Let us now suppose that $G$ is a Lie group. A vector $v \in V$ is said to be differentiable (under $\pi$ ) if the mapping $x \rightarrow \pi(x) v$ of $G$ into $V$ is of class $C^{\infty}$. Let $V^{\infty}$ denote the space of all differentiable vectors in $V . \mathrm{g}$ being the Lie algebra of $G$, define

$$
\pi(X) v=\lim _{t \rightarrow 0} t^{-1}(\pi(\exp t X) v-v) \quad\langle t \in \mathbf{R}, t \neq 0)
$$

for $X \in \mathfrak{g}$ and $v \in V^{\infty}$. Then $\pi(X)$ is a linear transformation in $V^{\infty}$ and $X \rightarrow \pi(X)$ is a representation of $g$ on $V^{\infty}$. Let $\left(\mathfrak{S}\right.$ be the universal enveloping algebra of $\mathfrak{g}_{c}$. Then this extends uniquely to a representation of $B S$ which we denote again by $\pi$.

As usual we regard elements of $(\mathfrak{S}$ as left-invariant differential operators on $G$. Define the anti-isomorphism $\varrho$ of $\mathfrak{G}$ onto the algebra of right-invariant differential operators on $G$ as in $[4(0), \S 2] . U$ being any open set in $G$, we write

$$
f(g ; x)=f(x ; \varrho(g)), \quad f\left(g ; x ; g^{\prime}\right)=f\left(x ; \varrho(g) \circ g^{\prime}\right) \quad\left(x \in U ; g, g^{\prime} \in(\mathfrak{S})\right.
$$

for $f \in C^{\infty}(U)$.

Lемма 2. Let $f \in C_{c}^{\infty}(G)$. Then $\pi(f) V \subset V^{\infty}$ and

for $X \in \mathfrak{g}$ and $v \in V$.

$$
\pi(X) \pi(f) v=-\pi(\varrho(X) f) v
$$

It is clear that

$$
\pi(y) \pi(f) v=\pi\left({ }_{y} f\right) v,
$$

where ${ }_{y} f(x)=f\left(y^{-1} x\right)$. Fix $X \in \mathfrak{g}$ and put $y_{t}=\exp t X(t \in \mathbf{R})$. Then

where $f^{\prime}=\varrho(X) f$. Hence

$$
f\left(y_{t}^{-1} x\right)-f(x)=-t \int_{0}^{1} f^{\prime}\left(y_{s t}^{-1} x\right) d s
$$

$$
t^{-1}\left(\pi\left(y_{t}\right)-1\right) \pi(f) w=-\int_{0}^{1} \pi\left(y_{s t}\right) v^{\prime} d s
$$

where $v^{\prime}=\pi\left(f^{\prime}\right) v$. The statements of the lemma are now obvious.

Let $g \rightarrow g^{*}$ be the anti-automorphism of (S) such that $X^{*}=-X(X \in \mathfrak{g})$. 
Corolla Y 1. $\pi(g) \pi(f) v=\pi\left(\varrho\left(g^{*}\right) f\right) v$ for $g \in$ (S).

This is obvious from the lemma.

Corollary 2. Let $v \in V^{\infty}$ and $f \in C_{c}^{\infty}(G)$. Then

$$
\pi(f) \pi(g) v=\pi\left(g^{*} f\right) v \quad(g \in(\mathfrak{S})
$$

Define $f_{y}(x)=f\left(x y^{-1}\right)(x \in G)$ for a fixed $y \in G$. Then $\pi(f) \pi(y) v=\pi\left(f_{y}\right) v$ and from this we conclude, as in the proof of Lemma 2 that

$$
\pi(f) \pi(X) v=-\pi(X f) v \quad(X \in \mathfrak{g}) .
$$

Clearly this implies the required result.

Let $f_{j}(j \geqslant 1)$ be a sequence in $C_{c}^{\infty}(G)$. We say that it is a Dirac sequence if the following conditions hold. $f_{j} \geqslant 0$ and

$$
\int f_{j} d x=\mathbf{I}
$$

and, for any neighborhood $U$ of $1, \operatorname{Supp} f_{j} \subset U$ for all $j$ except a finite number. Let $K$ be a compact subgroup of $G$. It is obvious that there always exists a Dirac sequence $f_{j}(j \geqslant 1)$ such that $f_{j}\left(k x k^{-1}\right)=f_{j}(x)(k \in K, x \in G)$.

Lемма 3. Let $f_{j}(j \geqslant 1)$ be a Dirac sequence in $C_{c}{ }^{\infty}(G)$. Then

for every $v \in V$.

$$
\lim _{j \rightarrow \infty} \pi\left(f_{j}\right) v=v
$$

Fix $v \in V, \nu \in S$ and $\varepsilon>0$. Then we can choose a neighborhood $U$ of 1 in $G$ such that $v(\pi(x) v-v) \leqslant \varepsilon$ for $x \in U$. Now

$$
\pi\left(f_{j}\right) v-v=\int f_{j}(x)(\pi(x) v-v) d x
$$

and therefore $\nu\left(\pi\left(f_{j}\right) v-v\right) \leqslant \varepsilon$ if Supp $f_{j} \subset U$. This proves the lemma.

Corollary 1. $V^{\infty}$ is dense in $V$.

This is obvious from Lemmas 2 and 3.

COROLLARY 2. Fix $v_{0} \in V$ and let $V_{0}$ be the smallest closed subspace of $V$ containing $v_{0}$ which is stable under $\pi(G)$. Then elements of the form $\pi(f) v_{0}\left(f \in C_{c}^{\infty}(G)\right)$ are dense in $V_{0}$. 
Let $W$ be the space consisting of all elements of the form $\pi(f) v_{0}\left(f \in C_{c}^{\infty}(G)\right)$. It is obvious that $W$ is stable under $\pi(G)$. Therefore the same holds for $\mathrm{Cl}(W)$. But then since $v_{0} \in \mathrm{Cl}(W)$ from Lemma 3, our assertion follows.

Let $K$ be a compact subgroup of $G$ and $\mathcal{E}_{K}$ the set of all equivalence classes of finitedimensional irreducible representations of $K$. For any $\delta \in \mathcal{E}_{K}$, define a linear transformation $E_{\mathrm{b}}$ in $V$ by

$$
E_{\triangleright} v=d(\delta) \int_{K} \operatorname{conj} \xi_{\triangleright}(k) \cdot \pi(k) v d k \quad(v \in V)
$$

Here $d(\delta)$ is the degree and $\xi_{b}$ the character of $\delta$ and $d k$ is the normalized Haar measure of $K$. Then $E_{\mathrm{b}}$ is a continuous projection. Put $V_{\mathfrak{b}}=E_{\triangleright} V$.

Lемма 4. $\sum_{\delta \in \varepsilon_{K}} V_{b} \cap V^{\infty}$ ts dense in $V$.

We shall give a proof of this lemma in $\S 6$.

\section{§ 3. Absolute convergence of the Fourier series}

As before let $S$ be the set of all continuous seminorms on a complex locally convex space $V$, which we assume to be complete. Let $\left\{v_{j}\right\}_{j \in J}$ be an indexed family of elements of $V$. We say that the series

$$
\sum_{j \in J} v_{j}
$$

converges, if the following condition holds. Define

$$
s_{F}=\sum_{j \in F} v_{j}
$$

for any finite subset $F$ of $J$. Then for any neighborhood $V_{0}$ of zero in $V$, there should exist a finite subset $F_{0}$ of $J$ such that $s_{F_{2}}-s_{F_{2}} \in V_{0}$ for any two finite subsets $F_{1}, F_{2}$ of $J$ containing $F_{0}$. Since $V$ is complete, the partial sums $s_{F}$ then have a limit $s$ in $V . s$ is called the sum of the series and we write

$$
s=\sum_{j \in J} v_{j}
$$

Moreover, the series is said to converge absolutely if

$$
\sum_{j \in J} v\left(v_{j}\right)<\infty
$$

for every $v \in S$. It is obvious that absolute convergence implies convergence.

Let $\pi$ be a representation of a compact Lie group $K$ on $V$ and define $E_{\mathfrak{b}}\left(\delta \in \mathcal{E}_{K}\right)$ as in $\S 2$. Then for any vector $v \in V$, we call $E_{b} v$ the bth Fourier component of $v$. 
L未мma 5. Let $v$ be a differentiable vector in $V$. Then the Fourier series

converges absolutely to $v$.

$$
\sum_{D \in \varepsilon_{K}} E_{b} v
$$

Let $\mathfrak{f}$ be the Lie algebra of $K$ and $\mathfrak{K}$ the universal enveloping algebra of $\mathfrak{f}_{c}$. Since $K$ is compact, we can choose a positive-definite quadratic form $Q$ on $\mathfrak{t}$ which is invariant under the adjoint representation of $K$. Let $X_{1}, \ldots, X_{r}$ be a base for $\mathfrak{l}$ over $\mathbf{R}$ orthonormal with respect to $Q$ and put

$$
\Omega=1-\left(X_{1}^{2}+\ldots+X_{r}^{2}\right) \in \mathfrak{R}
$$

Also put $\alpha_{\mathfrak{b}}=d(\mathfrak{D})$ conj $\xi_{\mathfrak{b}}\left(\delta \in \mathcal{E}_{K}\right)$ so that $E_{\mathfrak{b}}=\pi\left(\alpha_{\mathfrak{b}}\right)$. It is obvious that $\Omega$, regarded as a differential operator, commutes with both left and right translations of $K$. Fix a unitary representation $\sigma$ in the class $\delta$. Then $\sigma(\Omega)$ commutes with $\sigma(k)(k \in K)$ and therefore, by Schur's lemma, $\sigma(\Omega)=c(\delta) \sigma(1)$ where $c(\delta) \in \mathbf{C}$. However $\sigma\left(X_{i}\right)(1 \leqslant i \leqslant r)$ are obviously skew-adjoint operators. Hence $c(\delta)$ is real and $\geqslant 1$. Therefore

$$
\xi_{\triangleright}(k ; \Omega)=\operatorname{tr}(\sigma(k) \sigma(\Omega))=c(\delta) \xi_{\triangleright}(k) \quad(k \in K)
$$

and this shows that $\Omega \alpha_{b}=c(\mathfrak{D}) \alpha_{\mathrm{D}}$. Hence we conclude from Corollary 2 of Lemma 2 that

$$
E_{\mathrm{\jmath}} \pi(\Omega) v=c(\delta) E_{\mathrm{b}} v \quad\left(\delta \in \mathcal{E}_{K}\right) .
$$

LEMMA 6. Fix $\nu \in S$. Then we can select $\nu_{0} \in S$ such that

$$
\nu\left(E_{\mathfrak{b}} v\right) \leqslant c(\delta)^{-m} d(\mathfrak{\complement})^{2} \nu_{0}\left(\pi\left(\Omega^{m}\right) v\right)
$$

for $\mathfrak{D} \in \mathcal{E}_{K}$, any integer $m \geqslant 0$ and any differentiable vector $v \in V$.

Since $K$ is compact, we can choose $\nu_{0} \in S$ such that

for $k \in K$ and $u \in V$. Therefore

$$
v(\pi(k) u) \leqslant v_{0}(u)
$$

$$
\nu\left(E_{\mathrm{b}} u\right)=\nu\left(\pi\left(\alpha_{\mathrm{D}}\right) u\right) \leqslant d(\delta)^{2} v_{0}(u)
$$

since $\sup \left|\alpha_{\delta}\right| \leqslant d(\delta)^{2}$. Now we have seen above that

$$
E_{\mathfrak{b}} v=c(\mathfrak{\complement})^{-m} E_{\mathfrak{b}} \pi\left(\Omega^{m}\right) v \quad(m \geqslant 0),
$$

if $v$ is differentiable. Hence our assertion follows immediately.

LEMMA 7 .

if $m$ is sufficiently large.

$$
\sum_{\delta \in \varepsilon_{K}} d(\delta)^{2} c(\mathfrak{D})^{-m}<\infty
$$


Assuming this for a moment, we shall first finish the proof of Lemma 5. It is obvious from Lemmas 6 and 7 that the series

$$
\sum_{D} E_{b} v
$$

converges absolutely. Let $v_{0}$ denote its sum and put $u=v-v_{0}$. We have to show that $u=0$. Fix $\mathfrak{D}_{0} \in \mathcal{E}_{K}$. Since $E_{\downarrow_{0}}$ is continuous,

$$
E_{\mathrm{D}_{\mathrm{o}}} v_{\mathrm{0}}=\sum_{\mathrm{D}} E_{\mathrm{D}_{\mathrm{a}}} E_{\mathrm{b}} v=E_{\mathrm{D}_{\mathrm{a}}} v
$$

from the Schur orthogonality relations. This shows that $E_{\mathrm{b}} u=0$ for all $\delta \in \mathcal{E}_{K}$.

Now fix $\nu \in S$ and select $\nu_{0} \in S$ as in the proof of Lemma 6. For a given $\varepsilon>0$, choose a neighborhood $K_{0}$ of 1 in $K$ such that $v(\pi(k) u-u) \leqslant \varepsilon$ for $k \in K_{0}$. Fix a function $f \geqslant 0$ in $C(K)$ such that $f=0$ outiside $K_{0}$ and

$$
\int f(k) d k=1
$$

Then

$$
\nu(\pi(f) u-u) \leqslant \int f(k) \nu(\pi(k) u-u) d k \leqslant \varepsilon .
$$

Call a function $\beta \in C(K) K$-finite, if the space spanned by the left and right translates of $\beta$ under $K$ has finite dimension. Then by the Peter-Weyl theorem, we can choose a $K$-finite function $\beta \in C(K)$ such that $\sup |\beta-f| \leqslant \varepsilon$. Then

and therefore

$$
\nu(\pi(f) u-\pi(\beta) u) \leqslant \varepsilon \sup _{k \in K} \nu(\pi(k) u) \leqslant \varepsilon \nu_{0}(u)
$$

$$
v(\pi(\beta) u-u) \leqslant \varepsilon\left(v_{0}(u)+1\right)
$$

On the other hand, $\alpha_{\mathfrak{b}} * \beta=\beta * \alpha_{\S}$ since $\alpha_{\S}$ is a class function. Therefore

$$
E_{\mathrm{b}} \pi(\beta) u=\pi(\beta) E_{\mathrm{b}} u=0
$$

Moreover, since $\beta$ is $K$-finite, we can choose a finite subset $F$ of $\mathcal{E}_{K}$ such that

$$
\beta=\sum_{D \in F} \alpha_{\delta} * \beta
$$

Therefore

$$
\pi(\beta) u=\sum_{D \in F} E_{\triangleright} \pi(\beta) u=0
$$

and this shows that

$$
v(u) \leqslant \varepsilon\left(v_{0}(u)+1\right) .
$$

Making $\varepsilon$ tend to zero, we get $v(u)=0$. Since $v$ was an arbitrary element in $S$, this implies that $u=0$. 


$$
\sum_{\delta \in \mathcal{F}} c(\delta)^{-m} d(\delta)^{2} \leqslant 2^{m} \sum_{\lambda \in L^{*}}(1+q(\lambda))^{-m}|p(\lambda)|^{2}<\infty
$$

provided $m$ is sufficiently large.

\section{\$ 5. Differentiable vectors and Fourier series in function spaces}

If $M$ is a differentiable manifold, the spaces $C_{c}^{\infty}(M)$ and $C^{\infty}(M)$, taken with their usual topologies, are locally convex and complete. Let us now return to the notation of $\S 2$ and for any $f \in C^{\infty}(G)$ and $y \in G$, define $l(y) f$ to be the function $x \rightarrow f\left(y^{-1} x\right)(x \in G)$. We claim $l$ is a representation of $G$ on $C^{\infty}(G)$. For any compact subset $\Omega$ of $G$ and $g \in \mathscr{S}$, put

$$
v_{\Omega, \sigma}(f)=\sup _{\Omega}|g f| \quad\left(f \in C^{\infty}(G)\right)
$$

Then the seminorms $\nu_{\Omega, o}$, taken toghether for all $\Omega$ and $g$, define the topology of $C^{\infty}(G)$ and it is clear that

$$
\nu_{\Omega . g}(l(y) f)=v_{y-1, \Omega, g}(f)
$$

Moreover, $g$ and $l(y)$ commute as linear transformations in $C^{\infty}(G)$. Therefore if $f_{1}=g f$,

$$
\nu_{\Omega, g}(l(y) f-f)=\sup _{\Omega}\left|l(y) f_{1}-f_{1}\right| \rightarrow 0
$$

as $y \rightarrow 1$. Hence we conclude from Lemma 1 that $l$ is a representation.

LEмма 8. Fix $f \in C^{\infty}(G)$. Then $f$ is a differentiable vector under $l$ and $l(X) f=-\varrho(X) f$ for $X \in \mathfrak{g}$.

Fix $X \in g$ and put $y_{t}=\exp t X(t \in \mathbf{R})$. Then it would be enough to verify that

$$
\lim _{t \rightarrow 0} t^{-1}\left(l\left(y_{t}\right) f-f\right)=-\varrho(X) f
$$

in $C^{\infty}(G)$. Fix $\Omega$ and $g$ as above and put $f_{1}=g f$. Then since $g$ and $\varrho(X)$ commute, we have

$$
\nu_{\Omega . \Omega}\left(t^{-1}\left\{l\left(y_{t}\right) f-f\right\}+\varrho(X) f\right)=\sup _{\Omega}\left|t^{-1}\left\{l\left(y_{t}\right) f_{1}-f_{1}\right\}+f_{2}\right|
$$

where $f_{2}=\varrho(X) f_{1}$. But we have seen during the proof of Lemma 2 that

$$
f_{1}\left(y_{t}^{-1} x\right)-f_{1}(x)=-t \int_{0}^{1} f_{2}\left(y_{s t}^{-1} x\right) d s \quad(x \in G)
$$

Therefore

$$
\sup _{\Omega}\left|t^{-1}\left\{l\left(y_{t}\right) f_{1}-f_{1}\right\}+f_{2}\right| \leqslant \int_{0}^{1} \sup _{\Omega}\left|l\left(y_{s t}\right) f_{2}-f_{2}\right| d s \rightarrow 0
$$

as $t \rightarrow 0$. 


\section{\$ 4. Proof of Lemma 7}

It remains to verify Lemma 7. Let $K_{0}$ be the connected component of 1 in $K$. Since $K$ is compact, the index $N=\left[K: K_{0}\right]$ is finite. Put $\mathcal{E}=\mathcal{E}_{K}$ and let $\mathcal{E}_{0}$ be the set of all equivalence classes of irreducible finite-dimensional representations of $K_{0}$. If $\delta \in \mathcal{E}$ and $\delta_{0} \in \mathcal{E}_{0}$, we denote by $\left[\delta: \delta_{0}\right]$ the number of times $\mathfrak{D}_{0}$ occurs in the reduction of $\delta$ with respect to $K_{0}$. For a given $\delta_{0} \in \mathcal{E}_{0}$, let $\mathcal{E}\left(\delta_{0}\right)$ denote the set of all $\delta \in \mathcal{E}$ such that $\left[\delta: \delta_{0}\right] \geqslant 1$. Then it is a simple consequence of the Frobenius reciprocity theorem that

$$
\sum_{\delta \in \varepsilon\left(D_{0}\right)}\left[\mathrm{D}: \delta_{0}\right] d(\delta)=N d\left(\delta_{0}\right)
$$

where $d\left(\mathfrak{D}_{0}\right)$ is the degree of $\delta_{0}$. Let $\xi_{\delta_{0}}$ denote the character (on $K_{0}$ ) of a class $\mathfrak{D}_{0} \in \mathcal{E}_{0}$. Then it is easy to see that

$$
\Omega \xi_{\delta_{0}}=c\left({\mathfrak{D _ { 0 }}}_{0}\right) \xi_{D_{0}}
$$

where $c\left(\mathfrak{D}_{0}\right)=c(\delta)$ for any $\delta \in \mathcal{E}\left(\mathfrak{D}_{0}\right)$. Therefore

$$
\sum_{D \in \mathcal{E}} c(\delta)^{-m} d(\delta)^{2} \leqslant \sum_{D_{0} \in \varepsilon_{0}} c\left(\delta_{0}\right)^{-m} \sum_{D \in \mathcal{\varepsilon}\left(\mathfrak{D}_{0}\right)} d\left(\delta_{0}\right)^{2} \leqslant N^{2} \sum_{D_{0} \in \varepsilon_{0}} c\left(\delta_{0}\right)^{-m} d\left(\delta_{0}\right)^{2}
$$

Hence it would be enough to consider the case when $K$ is connected.

Fix a Cartan subgroup $A$ of $K$ with Lie algebra $\mathfrak{a}$. Then $A$ is connected. Let $L$ be the kernel of the exponential mapping of $\mathfrak{a}$ into $A$. Then $L$ is a lattice in a. Consider the space $\mathfrak{F}$ of all real-valued linear functions on $(-1)^{\frac{1}{2}} \mathfrak{a}$ and the lattice $L^{*}$ of all $\lambda \in \mathfrak{F}$ such that $e^{\lambda(H)}=1$ for $H \in L$. Introduce an order in $\mathfrak{F}$ and put

$$
Q=\frac{1}{2} \sum_{\alpha>0} \alpha
$$

where $\alpha$ runs over all positive roots of $(\mathfrak{f}, \mathfrak{a})$. For any $\delta \in \mathcal{E}$, let $\lambda(\mathfrak{D})$ denote the highest weight of $\delta$ with respect to $\mathfrak{a}$. Then the following facts are well known. $\lambda(\delta) \in L^{*}$ and $\delta$ is completely determined by $\lambda(\delta)$. Moreover there exists a polynomial function $p$ on $\mathfrak{F}$ such that $d(\delta)=p(\lambda(\delta))$ for all $\delta \in \mathcal{E}$. Finally, there exists a positive-definite quadratic form $q$ on $\mathfrak{F}$ such that

$$
c(\mathfrak{D})=1+q(\lambda(\delta)+\varrho)-q(\varrho) \quad(\delta \in \mathcal{E})
$$

We can obviously choose a compact set $C$ in $\mathfrak{F}$ such that

$$
q(\lambda+\varrho)-q(\varrho) \geqslant q(\lambda) / 2
$$

for $\lambda \in \mathfrak{F}$ outside $C$. Let $F$ denote the set of all $\delta \in \mathcal{E}$ such that $\lambda(\delta) \in C$. Then $F$ is finite since $L^{*} \cap C$ is finite. Let ${ }^{c} F$ denote the complement of $F$ in $\mathcal{E}$. Then 
For any $\alpha \in C(K)$ and $f \in C^{\infty}(G)$, define

$$
\alpha * f=\int_{E} \alpha(k) l(k) f d k
$$

and let $\alpha_{\delta}\left(\delta \in \mathcal{E}_{K}\right)$ have the same meaning as in $\S 3$.

Corollaky 1. The series

$$
\sum_{D \in \varepsilon_{\boldsymbol{K}}} \alpha_{\triangleright} * f \quad\left(f \in C^{\infty}(G)\right)
$$

converges absolutely to $f$ in $C^{\infty}(G)$.

This is an immediate consequence of Lemmas 5 and 8.

Corollary 2. Fix $f \in C_{c}^{\infty}(G)$. Then the series

converges absolutely to $f$ in $C_{c}{ }^{\circ}(G)$.

$$
\sum_{D \in \varepsilon_{\boldsymbol{I}}} \alpha_{D} * f
$$

Fix a compact set $\Omega$ in $G$ such that $K \Omega=\Omega$ and Supp $f \subset \Omega$. As usual, let $C_{\Omega}{ }^{\infty}(G)$ denote the space of all functions $\phi \in C_{c}{ }^{\infty}(G)$ whose support lies in $\Omega$. Then $C_{\Omega}{ }^{\infty}(G)$ is a closed subspace of $C_{c}^{\infty}(G)$ and the two topologies induced on it from $\mathrm{C}^{\infty}(G)$ and $C_{c}{ }^{\infty}(G)$ coincide. Therefore our assertion follows from Corollary 1.

Let $r(y) f\left(y \in G, f \in C^{\infty}(G)\right)$ denote the function $x \rightarrow f(x y)(x \in G)$. Then one proves in the same way that $r$ is a representation of $G$ on $C^{\infty}(G)$ and every $f \in C^{\infty}(G)$ is differentiable under $r$. Moreover $l(g) f=\varrho\left(g^{*}\right) f$ and $r(g) f=g f(g \in \mathbb{S})$ in the notation of the corollaries of Lemma 2. Define

$$
f * \alpha=\int_{E} \alpha\left(k^{-1}\right) r(k) f d k \quad(\alpha \in C(K))
$$

Then the analogues of the two corollaries of Lemma 8 hold also for $r$.

Note that $l(x)$ and $r(y)(x, y \in G)$ commute and hence

$$
\alpha *(f * \beta)=(\alpha * f) * \beta \quad(\alpha, \beta \in C(K)) .
$$

We may therefore simply write $\alpha * f * \beta$.

Define a representation $\pi$ of $G \times G$ on $C^{\infty}(G)$ as follows.

$$
\pi((x, y)) f=l(x) r(y) f \quad\left(x, y \in G, f \in C^{\infty}(G)\right) .
$$

Since

$$
\pi((x, y)) f-f=l(x)(r(y) f-f)+(l(x) f-f)
$$


it follows from Lemma 1 that $\pi$ is indeed a representation. It is obvious from Lemma 8 and its analogue for $r$, that every $f \in C^{\infty}(G)$ is differentiable under $\pi$.

LIммA 9. Let $V$ be either one of the two spaces $C^{\infty}(G)$ or $C_{c}^{\infty}(G)$, taken with its usual topology. Then for any $f \in V$, the series

converges absolutely to $f$ in $V$.

$$
\sum_{D_{1}, D_{2} \in \varepsilon_{K}} \alpha_{D_{1}} * f * \alpha_{D_{2}}
$$

If $V=C^{\infty}(G)$, this follows immediately from Lemma 5 . The rest is proved in the same way as Corollary 2 of Lemma 8.

\section{§6. Proof of Lemma 4}

We now come to the proof of Lemma 4. Fix $v \in V, \nu \in S$ and $\varepsilon>0$. Then, by Lemma 3 , we can select $f \in C_{c}{ }^{\infty}(G)$ such that

$$
v(\pi(f) v-v) \leqslant \varepsilon
$$

Choose a compact set $\Omega$ in $G$ such that $K \Omega=\Omega$ and Supp $f \subset \Omega$. Put

$$
\mu(g)=\int|g| d x \quad\left(g \in C_{c}^{\infty}(G)\right)
$$

and

$$
\alpha_{F}=\sum_{\delta \in F} \alpha_{\delta}
$$

for any finite subset $F$ of $\mathcal{E}_{K}$. Then $\mu$ is a continuous seminorm on $C_{c}^{\infty}(G)$ and $\operatorname{Supp}\left(f-\alpha_{F} * f\right) \subset \Omega$. Therefore

$$
v\left(\pi\left(f-\alpha_{F} * f\right) v\right) \leqslant c \mu\left(f-\alpha_{F} * f\right)
$$

where

$$
c=\sup _{x \in \Omega} \nu(\pi(x) v)<\infty
$$

Hence we conclude from Corollary 2 of Lemma 8 that

$$
v\left(\pi\left(f-\alpha_{F} * f\right) v\right) \leqslant \varepsilon
$$

if $F$ is sufficiently large. Therefore

$$
v\left(\pi\left(\alpha_{F} * f\right) v-v\right) \leqslant v\left(\pi\left(f-\alpha_{F} * f\right) v\right)+\nu(\pi(f) v-v) \leqslant 2 \varepsilon .
$$

Since

$$
\pi\left(\alpha_{F} * f\right) v \in \sum_{D \in \varepsilon_{E}} V_{D} \cap V^{\infty}
$$

from Lemma 2, the assertion of Lemma 4 is now obvious. 


\section{§ 7. Some elementary facts about $\sigma$ and $\Xi$}

Let $\mathfrak{g}$ be a reductive Lie algebra over $\mathbf{R}$. Then $\mathfrak{g}=\mathfrak{c}+\mathfrak{g}_{1}$ where $\mathfrak{c}$ is the center and $g_{1}$ the derived algebra of $g$. Let $\theta$ be an automorphism of $g$ such that $\theta^{2}=1$ and let $f$ and $\mathfrak{p}$ be the subspaces of $\mathfrak{g}$ corresponding to the eigenvalues 1 and -1 respectively of $\theta$. We assume that the quadratic form

$$
Q(X)=-\operatorname{tr}(\operatorname{ad} X \operatorname{ad} \theta(X)) \quad(X \in \mathfrak{g})
$$

is positive-definite on $\mathfrak{g}_{1}$.

Let $G$ be a connected Lie group with Lie algebra $g$ and $K$ the analytic subgroup of $G$ corresponding to $\mathfrak{f}$. We assume that:

1) $K$ is compact,

2) The mapping $(k, X) \rightarrow k \exp X(k \in K, X \in \mathfrak{p})$ defines an analytic diffeomorphism of $K \times \mathfrak{p}$ onto $G$.

Let $\log$ denote the inverse of the exponential mapping from $\mathfrak{p}$ to exp $\mathfrak{p}$. Suppose we have a Euclidean norm on $\mathfrak{p}$ such that $\left\|X^{k}\right\|=\|X\|(k \in K, X \in \mathfrak{p})$ and

$$
\left\langle X,(\operatorname{ad} Z)^{2} Y\right\rangle=\left\langle(\operatorname{ad} Z)^{2} X, Y\right\rangle \quad(X, Y, Z \in \mathfrak{p})
$$

for the corresponding scalar product. Then it is easy to see that $\mathfrak{c}_{\mathfrak{p}}=\mathfrak{c} \cap \mathfrak{p}$ and $\mathfrak{p}_{1}=[\mathfrak{f}, \mathfrak{p}]=$ $\mathfrak{p} \cap \mathfrak{g}_{1}$ are mutually orthogonal (under this norm). Put

$$
\sigma(x)=\|X\|
$$

for $x=k \exp X(k \in K, X \in p)$ and extend $\theta$ to an automorphism of $G$ (see [4(o), § 16]). Then it is obvious that

$$
\sigma(x)=\sigma(\theta(x))=\sigma\left(x^{-1}\right) \quad(x \in G) .
$$

If $|C|\left(C \epsilon_{\mathfrak{p}}\right)$ is an arbitrary Euclidean norm on $\mathfrak{c}_{\mathfrak{p}}$ and we define

$$
\|C+X\|^{2}=|C|^{2}-\operatorname{tr}(\operatorname{ad} X \operatorname{ad} \theta(X)) \quad\left(C \epsilon_{\mathfrak{c}_{p}}, X \in_{p_{1}}\right)
$$

then all the above conditions are fulfilled.

Lem ма $10 . \sigma(x y) \leqslant \sigma(x)+\sigma(y)$ for $x, y \in G$.

We may obviously assume that $x=\exp X, y=\exp Y(X, Y \in \mathfrak{p})$. Then if $x y=k \exp Z$ $(k \in K, Z \in \mathfrak{p})$, it is clear that

$$
\exp 2 Z=\theta(x y)^{-1} x y=\exp Y \cdot \exp 2 X \cdot \exp Y .
$$


Now define $Z(t) \in \mathfrak{p}$ by

$$
\exp 2 Z(t)=\exp t Y \cdot \exp 2 X \cdot \exp t Y \quad(t \in \mathbf{R}) .
$$

Then $Z(t)$ is an analytic function of $t$ and it follows by differentiating with respect to $t$ that (see $[5(\mathrm{a})$, p. 95])

$$
\{(1-\exp (-2 \operatorname{ad} Z(t))) / 2 \operatorname{ad} Z(t)\} Z^{\prime}(t)=\frac{1}{2}(1+\exp (-2 \operatorname{ad} Z(t))) Y,
$$

where $Z^{\prime}(t)=d Z(t) / d t$. Therefore

$$
\left\langle Z(t), Z^{\prime}(t)\right\rangle=\langle Z(t), Y\rangle
$$

Now suppose $Z(t)=0$ for some $t \in \mathbf{R}$. Then

$$
1=\exp t Y \cdot \exp 2 X \cdot \exp t Y
$$

and therefore $X=-t Y$. But then $Z=(1-t) Y$ and so

$$
\|Z\| \leqslant\|Y\|+\|t Y\|=\|Y\|+\|X\|
$$

which is equivalent to the assertion of the lemma. Hence we may assume that $Z(t) \neq 0$ for every $t \in \mathbf{R}$. Then $\|Z(t)\|$ is analytic in $t$ and

$$
\left\langle Z(t), Z^{\prime}(t)\right\rangle=\|Z(t)\| d\|Z(t)\| / d t .
$$

This shows that

$$
\|Z(t)\| d\|Z(t)\| / d t=\langle Z(t), Y\rangle
$$

and hence

$$
|d\|Z(t)\| / d t| \leqslant\|Y\|
$$

But then by integrating we get

$$
\|Z(1)\|-\|Z(0)\| \leqslant\|Y\| \text {. }
$$

However $Z(0)=X$ and $Z(1)=Z$. Therefore

and this proves the lemma.

$$
\|Z\| \leqslant\|X\|+\|Y\|
$$

Put $\mathfrak{l}_{1}=\mathfrak{l}+\mathfrak{c}=\mathfrak{l}+\mathfrak{c}_{\mathfrak{p}}$ and let $K_{1}$ be the analytic subgroup of $G$ corresponding to $\mathfrak{l}_{1}$ Define the function $\Xi$ on $G$ corresponding to $K_{1}$ as in [4(q), § 16]. Then by [4 (q), Lemma 31] $\Xi$ is everywhere positive.

LеммА 11. There exists a number $r \geqslant 0$ such that

$$
\int_{G} \Xi(x)^{2}(1+\sigma(x))^{-r} d x<\infty
$$


Let $G_{0}$ and $C_{\mathfrak{p}}$ be the analytic subgoups of $G$ corresponding to $\dot{\mathfrak{g}}_{0}=\mathfrak{f}+\mathfrak{p}_{1}$ and $\mathfrak{c}_{\mathfrak{p}}$ respectively. Then since $\Xi(y c)=\Xi(y)$ and

$$
\sigma(y c) \geqslant \max (\sigma(y), \sigma(c)) \quad\left(y \in G_{0}, c \in C_{\mathfrak{p}}\right),
$$

it is clear that the above integral is majorized by

$$
\int_{C_{\mathfrak{p}}}(1+\sigma(c))^{-r / 2} d c \int_{G_{\mathfrak{o}}} \Xi(y)^{2}(1+\sigma(y))^{-r / 2} d y,
$$

where $d c$ and $d y$ are the Haar measures on $C_{\mathfrak{p}}$ and $G_{0}$ respectively. Now it is clear that

$$
\int_{C_{\mathfrak{p}}}(1+\sigma(c))^{-r / 2} d:<\infty
$$

if $r$ is sufficiently large. Hence it would be enough to consider the case when $\mathfrak{c}_{\mathfrak{p}}=\{0\}$. Then we can obviously replace $G$ by $G / Z_{G}$ where $Z_{G}$ is the center of $G$. So we may assume that $G$ is semisimple. Define $\mathfrak{a}, \Sigma, \mathfrak{a}^{+}, A$ and $A^{+}$as in $[4(\mathrm{q}), \S 21]$ and let $d h$ denote the Haar measure of $A$. Then it follows from [4(d), Lemma 38] that

$$
\int_{G} \Xi(x)^{2}(1+\sigma(x))^{-r} d x=c_{1} \int_{A^{+}} D(h) \Xi(h)^{2}(1+\sigma(h))^{-r} d h,
$$

where $c_{1}$ is a positive number and

$$
D(\exp H)=\prod_{\alpha \in \Sigma}\left(e^{\alpha(H)}-e^{-\alpha(H)}\right)^{m_{\alpha}} \quad\left(H \in \mathfrak{a}^{+}\right)
$$

$m_{\alpha}$ being the multiplicity of $\alpha$. Put $\varrho=\frac{1}{2} \sum_{\alpha \in \Sigma} m_{\alpha}$. Then we know from [4(j), Theorem"3] that we can choose positive numbers $c_{2}$ and $d$ such that

$$
\Xi(h) \leqslant c_{2} e^{-\varrho(\log h)}(1+\sigma(h))^{d} \quad\left(h \in A^{+}\right) .
$$

Therefore, since it is clear that

we conclude that

$$
D(h) \leqslant e^{2 \varrho(\log h)} \quad\left(h \in A^{+}\right),
$$

$$
\int_{G} \Xi^{2}(1+\sigma)^{-r} d x \leqslant c_{1} c_{2}^{2} \int_{A^{+}}(1+\sigma(h))^{2 d-r} d h<\infty,
$$

if $r$ is sufficiently large. This proves Lemma 11 .

Remark. Suppose $\mathfrak{c}_{\mathfrak{p}}=\{0\}$. Then one proves in the same way that $(1+\sigma)^{r} \Xi \in L_{p}(G)$ for $p>2$ and $r \in \mathbf{R}$.

2-662900 Acta mathematica. 116. Imprimé le 10 juin 1966. 


\section{§ 8. Proof of Theorem 1}

We keep to the notation of $\S 7$ and define $\mathbb{S} \xi$ as in $\S 2$. Let 8 be the center of $\mathbb{S}$ and $\mathfrak{\Re}$ the subalgebra of $\left(\mathscr{S}\right.$ generated by $\left(1, \mathfrak{f}_{c}\right)$. The following theorem will play an important role in the harmonic analysis on $G$.

THEOREM 1. (1) Let $V$ be a complex vector space of finite dimension and $f$ a $C^{\infty}$ function from $G$ to $V$ such that the functions $z f\left(z \in Z_{\mathfrak{I}}\right)$ span a finite-dimensional space. Fix a neighborhood $U$ of 1 in $G$ and let $J$ be the space of all functions $\alpha \in C_{c}^{\infty}(G)$ such that Supp $\alpha \subset U$ and $\alpha\left(k x k^{-1}\right)=\alpha(x)(k \in K, x \in G)$. Then there exists an element $\alpha \in J$ such that $f * \alpha=f$.

We regard $f$ as an element of $\mathrm{C}^{\infty}(G) \otimes V$ and extend the representation $r$ of $\S 5$ on this space by making $G$ act trivially on $V$. Then, as we have seen in $\S 5$, every element $\phi \in C^{\infty}(G) \otimes V$ is differentiable under $r$ and $r(g) \phi=g \phi(g \in(S)$. Let $\mathfrak{U}$ be the set of all $u \in \mathbb{Z} \Re$ such that $u f=0$. Then $\mathfrak{U}$ is a left ideal in $8 \mathfrak{N}$ of finite codimension. Let $W$ be the smallest closed subspace of $C^{\infty}(G) \otimes V$ containing $f$, which is stable under $r(G)$. Then it is obvious that $W$ contains $W_{0}=r(\mathbb{S}) f$. We claim that $W=\mathrm{Cl}\left(W_{0}\right)$. For otherwise, by the HahnBanach theorem, we could choose a continuous linear function $\beta \neq 0$ on $W$ such that $\beta=0$ on $W_{0}$. Put

$$
F(x)=\beta(r(x) f) \quad(x \in G)
$$

Since $f$ is differentiable under $r$, it is obious that $F \in C^{\infty}(G)$ and

$$
F(x ; g)=\beta(r(x) r(g) f) \quad(g \in(\mathfrak{S}) .
$$

Therefore $u F=0$ for $u \in \mathfrak{U}$. However $\mathfrak{U}$ contains elliptic differential operators (see the proof of Lemma 33 of $[4(q)]$ ) and so we conclude that $F$ is an analytic function. On the other hand,

$$
F(1 ; g)=\beta(r(g) f)=0 \quad(g \in(\mathfrak{S})
$$

since $\beta=0$ on $W_{0}$. Hence $F=0$ and this implies that $\beta=0$ on $W$. This contradiction proves that $W=\mathrm{Cl}\left(W_{0}\right)$.

Put $W_{1}=r(\mathfrak{\Re}) f$. Then $\operatorname{dim} W_{1}<\infty$ and therefore $W_{1}$ is closed in $W$. Moreover one proves in the same way as above that $r(K) f \subset W_{1}$ so that $W_{1}$ is stable under $r(K)$. Since $f \in W_{1}$, we can choose a finite subset $F$ of $\mathcal{E}_{K}$ such that $f=f * \alpha_{F}$. (Here $\alpha_{F}$ has the same meaning as in $\S$ 6.) Put

( $\left.{ }^{1}\right)$ In my original proof of this theorem, I had to impose a mild condition on $f$ at infinity, in order to get a representation of $G$ on a suitable Banach space containing $f$. It was noticed by $H$. Jacquet that the argument worked equally well for a representation on a locally convex space and therefore the extra condition could be dropped. The proof given here, which is simpler than the original version, although based on the same idea, was obtained during a discussion with $\mathrm{A}$. Borel. 


$$
E_{F}=\sum_{\mathfrak{b} \in F} E_{\mathrm{b}}
$$

in the notation of $\S 2$ (with $\pi=r$ ). We claim that $W_{F}=E_{F} W$ has finite dimension. Since $W_{1}$ is fully reducible under $r(K)$, it is obvious that the natural representation of $\mathscr{K}$ on $\mathfrak{K} / \mathscr{K} \cap \mathfrak{U}$ is semisimple. Moreover since $\operatorname{dim}(3 \Re / \mathfrak{A})<\infty$, it follows from [4 (a), Theorem 1, p. 195] that

$$
W_{0}=\sum_{\delta \in \varepsilon_{B}} E_{\mathfrak{b}} W_{0}
$$

and $\operatorname{dim} E_{\mathrm{b}} W_{0}<\infty$ for every $\delta \in \mathcal{E}_{K}$. On the other hand, $W_{0}$ is dense in $W$ and therefore $E_{\mathrm{b}} W_{0}$ is dense in $E_{\mathrm{b}} W$. Hence $E_{\mathrm{b}} W_{0}=E_{\mathrm{b}} W$ and this shows that $\operatorname{dim} W_{F}<\infty$.

We have seen in $\S 2$ that there exists a Dirac sequence $\alpha_{j}(j \geqslant 1)$ with $\alpha_{j} \in J$. Then by Lemma $3, f * \alpha_{j} \rightarrow f$ in $W$ as $j \rightarrow \infty$. Let $W_{2}$ be the space of all elements in $W$ of the form $f * \alpha(\alpha \in J)$. Since $\alpha\left(k x k^{-1}\right)=\alpha(x)(k \in K, x \in G)$ and $f \in W_{F}$, it is obvious that $W_{2} \subset W_{F}$. Hence $W_{2}$ is a vector space of finite dimension and therefore it is closed in $W$. Therefore $f=\lim _{j \rightarrow \infty} f * \alpha_{j} \in W_{2}$ and this proves the theorem.

\section{§9. The space $C(G)$}

Fix an open set $U$ in $G$ and let $C^{\circ}(U)$ denote the space of all continuous functions $f$ from $U$ to $\mathbf{C}$ such that

$$
\nu_{r}(f)=\sup _{U}(1+\sigma)^{r} \Xi^{-1}|f|<\infty
$$

for every $r \in \mathbf{R}$. Put

$$
{ }_{g_{1}} \nu_{r, g_{2}}(f)=\nu_{r}\left(\left(\varrho\left(g_{1}\right) \circ g_{2}\right) f\right)
$$

for $f \in C^{\infty}(U), g_{1}, g_{2} \in(G)$ and $r \in \mathbf{R}$. Let $\mathrm{C}(U)$ be the subspace of those $f \in C^{\infty}(U)$ for which ${ }_{g_{2}} v_{r, g_{2}}(f)<\infty$ for all $r$ and $\left(g_{1}, g_{2}\right)$. We topologize $\mathcal{C}(U)$ by means of the seminorms $g_{g_{1}} v_{r, g_{2}}$ $\left(g_{1}, g_{2} \in(\mathfrak{S}, r \in \mathbf{R})\right.$. In this way $\mathrm{C}(U)$ becomes a locally convex Hausdorff space which is easily seen to be complete. $\left({ }^{1}\right)$

LFммA 12. Fix $a, b \in G$ and, for any function $f$ on $U$, let $f^{\prime}$ denote the function on $a U b$ given by

$$
f^{\prime}(x)=f\left(a^{-1} x b^{-1}\right) \quad(x \in a U b) .
$$

Then $f \rightarrow f^{\prime}$ defines a topological mapping of $\mathrm{C}(U)$ onto $\mathrm{C}(a U b)$.

This is an easy consequence of Lemma 10 and [4(q), Lemma 32].

Now let $G^{\prime}$ be a Lie group such that $G$ is the connected component of 1 in $G^{\prime}$. Moreover let $U$ be an open subset of $G^{\prime}$ which meets only a finite number of connected components of $G^{\prime}$. Then we can choose $a_{i} \in G^{\prime}$ and open sets $U_{i}$ in $G$ such that $U$ is the disjoint union

(1) $\mathrm{C}(U)=\{0\}$ by convention, if $U$ is empty. 
of $a_{i} U_{i}(1 \leqslant i \leqslant r)$. For any $f \in C^{\infty}(U)$, let $f_{i}$ denote the function on $U_{i}$ given by $f_{i}(x)=$ $f\left(a_{i} x\right)\left(x \in U_{i}\right)$. Consider the space $\mathrm{C}(U)$ of all $f \in C^{\infty}(U)$ such that $f_{i} \in \mathcal{C}\left(U_{i}\right)(1 \leqslant i \leqslant r)$ and let $V$ denote the Cartesian product of $\mathrm{C}\left(U_{i}\right)(1 \leqslant i \leqslant r)$ with the natural topology. We topologize $\mathrm{C}(U)$ in such a way that the mapping $f \rightarrow\left(f_{1}, \ldots, f_{r}\right)$ of $\mathrm{C}(U)$ onto $V$ becomes an isomorphism. It follows from Lemma 12 that the structure of $\mathrm{C}(U)$, as a locally convex space, is independent of the choice of $a_{i}$ and $U_{i}$. Moreover it is obvious that the injection of $C_{c}{ }^{\infty}(U)$ into $\mathrm{C}(U)$ is continuous.

By a tempered distributon $T$ on $U$, we mean a continuous linear mapping of $\mathrm{C}(U)$ into $\mathbf{C}$.

Now assume that $G^{\prime} / G$ is finite. Then $U$ can be any open subset of $G$.

THEOREM 2. Suppose $G^{\prime} / G$ is finite. Then $C_{c}{ }^{\infty}\left(G^{\prime}\right)$ is dense in $\mathrm{C}\left(G^{\prime}\right)$.

In view of this theorem, we can identify tempered distributions on $G^{\prime}$ with those distributions which are continuous in the relative topology of $C_{c}^{\infty}\left(G^{\prime}\right)$ as a subspace of $\mathrm{C}\left(G^{\prime}\right)$. Moreover, it is obviously enough to prove this theorem in case $G^{\prime}=G$. This requires some preparation which will be undertaken in the next few sections.

\section{§ 10. The left- and right-regular representations on $C(G)$}

Let $S$ denote the set of all continuous seminorms on $\mathrm{C}(G)$. For any $f \in \mathcal{C}(G)$ and $y \in G$, define $l(y) f$ and $r(y) f$ as in $\S 5$.

Lемм А 13. $l(y) f$ and $r(y) f$ are in $\mathrm{C}(G)$. Moreover for a given compact set $\Omega$ in $G$ and $\nu \in S$, we can choose $\nu^{\prime} \in S$ such that

for $y \in \Omega$ and $f \in \mathcal{C}(G)$.

$$
\boldsymbol{\nu}(l(y) f)+\nu(r(y) f) \leqslant \nu^{\prime}(f)
$$

Put $r(y) f=f_{y}$ and fix $g, g^{\prime} \in(S$. Then

$$
f_{y}\left(g^{\prime} ; x ; g\right)=f\left(g^{\prime} ; x y ; g^{y^{-1}}\right)
$$

for $x, y \in G$. We can choose linearly independent elements $g_{i}(1 \leqslant i \leqslant p)$ in $(S)$ and analytic functions $a_{i}$ on $G$ such that

$$
g^{y^{-1}}=\sum_{1 \leqslant i \leqslant p} a_{i}(y) g_{i} \quad(y \in G)
$$

Then

$$
f_{y}\left(g^{\prime} ; x ; g\right)=\sum_{i} a_{i}(y) f\left(g^{\prime} ; x y ; g_{i}\right)
$$

If we apply a similar argument to $l(y) f$ and take into account Lemma 10 and [4 (q), Lemma $32]$, our assertions follow immediately. 
LEMMA 14. Fix $s \geqslant 0$ and put

$$
v_{s}(f)=\sup (1+\sigma)^{s} \Xi^{-1}|f| \quad(f \in \mathbb{C}(G))
$$

Then for any $\varepsilon>0$, we can choose a neighborhood $U$ of 1 in $G$ and an element $\nu^{\prime} \in S$ such that

$$
v_{s}\left(f_{y}-f\right) \leqslant \varepsilon v^{\prime}(f)
$$

for $y \in U$ and $f \in \mathrm{C}(G)$. Moreover, $\nu^{\prime}$ does not depend on $\varepsilon$.

Introduce a norm in $g$ and fix a base $X_{1}, \ldots, X_{n}$ for $g$ over $\mathbf{R}$. Then we can choose $c_{0}>0$ such that

$$
\max _{1 \leqslant i \leqslant n}\left|c_{i}\right| \leqslant c_{0}\left|\sum_{1 \leqslant i \leqslant n} c_{i} X_{i}\right|
$$

for $c_{i} \in \mathbf{R}(1 \leqslant i \leqslant n)$. Now let $f \in C(G)$ and $X \in \mathrm{g}$. Then it is clear that

and therefore

$$
f(x \exp X)-f(x)=\int_{0}^{1} f(x \exp t X ; X) d t \quad(x \in G)
$$

$$
|f(x \exp X)-f(x)| \leqslant c_{0}|X| \sum_{i} \int_{0}^{1}\left|f\left(x \exp t X ; X_{i}\right)\right| d t
$$

But then it is obvious that we can choose $c>0$ such that

$$
v_{s}\left(f_{\exp X}-f\right) \leqslant c|X| \sum_{1 \leqslant i \leqslant n} v_{s}\left(X_{i} f\right)
$$

for $|X| \leqslant 1(X \in g)$ and $f \in C(G)$. Clearly this implies the assertion of the lemma.

CoRoLLARY 1. Fix $\nu \in S$ and $\varepsilon>0$. Then we can choose a neighborhood $U$ of 1 in $G$ and $v^{\prime} \in S$ such that

$$
v(l(y) f-f)+v(r(y) f-f) \leqslant \varepsilon v^{\prime}(f)
$$

for $y \in U$ and $f \in \mathcal{C}(G)$. Moreover, $v^{\prime}$ is independent of $\varepsilon$.

We use the notation of the proof of Lemma 13. Then

$$
f_{y}\left(g^{\prime} ; x ; g\right)-f\left(g^{\prime} ; x ; g\right)=\sum_{i}\left(a_{i}(y)-1\right) f\left(g^{\prime} ; x y ; g_{i}\right)+\left\{f\left(g^{\prime} ; x y ; g\right)-f\left(g^{\prime} ; x ; g\right)\right\}
$$

Put $D=\varrho\left(g^{\prime}\right) \circ g$ and $D_{i}=\varrho\left(g^{\prime}\right) \circ g_{i}$. Then $D$ and $D_{i}$ define continuous endomorphisms of $\mathrm{C}(G)$ and the above relation may be written as

$$
D(r(y) f-f)=\sum_{i}\left(a_{i}(y)-1\right) r(y) D_{i} f+(r(y)-1) D f .
$$

Therefore it is obvious from Lemmas 13 and 14 that, for a given $s \geqslant 0$, we can choose a neighborhood $U$ of 1 in $G$ and an element $\nu^{\prime} \in S$ (independent of $\varepsilon$ ) such that 


$$
\nu_{s}(D(r(y) f-f)) \leqslant \varepsilon \nu^{\prime}(f)
$$

for $y \in U$ and $f \in(C(G)$. Since a similar argument holds for $l(y)$, our assertion follows.

COROLLARY 2. Both $l$ and $r$ define representations of $G$ on $\mathcal{C}(G)$.

This is obvious from Lemmas 1 and 13 and Corollary 1 above.

LEMMA 15. Every element $f \in \mathrm{C}(G)$ is a differentiable vector under both $l$ and $r$. Moreover, $r(X) f=X f$ and $l(X) f=-\varrho(X) f$ for $X \in \mathrm{g}$.

Fix $f \in \mathcal{C}(G), Y \in \mathfrak{g}$ and put

$$
\phi_{t}=t^{-1}\left(r\left(y_{t}\right) f-f\right)-Y f \quad(t \in \mathbf{R}, t \neq 0),
$$

where $y_{t}=\exp t Y$. Then it follows from Lemma 8 that if $D$ is any differential operator on $G$ and $\Omega$ any compact subset of $G$, then

$$
\sup _{\Omega}\left|D \phi_{t}\right| \rightarrow 0
$$

as $t \rightarrow 0$. Fix $g, g^{\prime} \in(S)$ and a number $m \geqslant 0$. Then it follows in particular that for any compact $\Omega$,

$$
\sup _{x \in \Omega}\left|\phi_{t}\left(g^{\prime} ; x ; g\right)\right| \Xi(x)^{-1}(1+\sigma(x))^{m} \rightarrow 0
$$

as $t \rightarrow 0$.

On the other hand (see the proof of Lemma 2),

$$
\phi_{t}(x)=\int_{0}^{1}\left\{f\left(x y_{t s} ; Y\right)-f(x ; Y)\right\} d s .
$$

Hence

$$
\phi_{t}\left(g^{\prime} ; x ; g\right)=\sum_{i} \int_{0}^{1} a_{i}\left(y_{t s}\right) f^{\prime}\left(x y_{t s} ; g_{i} Y\right) d s-f^{\prime}(x ; g Y)
$$

where $f^{\prime}=\varrho\left(g^{\prime}\right) f$ and $a_{i}, g_{i}(1 \leqslant i \leqslant p)$ have the same meaning as in the proof of Lemma 13 . Fix a compact neighborhood $U=U^{-1}$ of 1 in $G$. Then we can choose a number $c$ such that $1+\sigma(y) \leqslant c,\left|a_{i}(y)\right| \leqslant c(1 \leqslant i \leqslant p)$ and $\Xi(x y) \leqslant c \Xi(x)$ for $y \in U$ and $x \in G$. Fix $\delta>0$ such that $y_{t} \in U$ for $|t| \leqslant \delta$. Then

$$
\begin{aligned}
& \left|\phi_{t}\left(g^{\prime} ; x ; g\right)\right| \Xi(x)^{-1}(1+\sigma(x))^{m} \\
& \quad \leqslant c^{m+2} \sum_{i} \sup _{u \in U}\left|f^{\prime}\left(x u ; g_{i} Y\right)\right| \Xi(x u)^{-1}(1+\sigma(x u))^{m}+\left|f^{\prime}(x ; g Y)\right| \Xi(x)^{-1}(1+\sigma(x))^{m}
\end{aligned}
$$

for $|t| \leqslant \delta$. Now fix $\varepsilon>0$. Since $f^{\prime} \in C(G)$, we can choose a compact set $\Omega_{0}$ in $G$ such that 


$$
\left|f^{\prime}\left(x ; g_{i} Y\right)\right| \Xi(x)^{-1}(1+\sigma(x))^{m} \leqslant \varepsilon
$$

outside $\Omega_{0}$ for $0 \leqslant i \leqslant p$. (Here $g_{0}=g$.) Put $\Omega=\Omega_{0} U$. Then $\Omega$ is compact and it is clear that

$$
\left|\phi_{t}\left(g^{\prime} ; x ; g\right)\right| \Xi(x)^{-1}(1+\sigma(x))^{m} \leqslant(p+1) c^{m+2} \varepsilon
$$

if $x \notin \Omega$ and $|t| \leqslant \delta$. Therefore, in view of our earlier result, we can now conclude that $\phi_{t} \rightarrow 0$ in $\mathcal{C}(G)$ as $t \rightarrow 0$. This shows that $f$ is differentiable under $r$ and $r(Y) f=Y f$. The proof for $l$ is similar.

Define $\alpha_{\mathfrak{D}}\left(\mathfrak{D} \in \mathcal{E}_{K}\right)$ as in $\S 5$.

LEMMA 16. For any $f \in \mathrm{C}(G)$, the series

$$
\sum_{D_{1}, \delta_{2} \in \varepsilon_{I}} \alpha_{D_{1}} * f * \alpha_{D_{2}}
$$

converges absolutely to $f$ in $\mathrm{C}(G)$.

This is proved in the same way as Lemma 9.

\section{\$ 11. Spherical functions}

Let $\mu=\left(\mu_{1}, \mu_{2}\right)$ be a (continuous) double representation ( $\left.{ }^{1}\right)$ of $K$ on a (complex) vector space $V$ of finite dimension. Then by a $\mu$-spherical function we mean a function $\phi$ from $G$ to $V$ such that

Fix a norm on $V$.

$$
\phi\left(k_{1} x k_{2}\right)=\mu_{1}\left(k_{1}\right) \phi(x) \mu_{2}\left(k_{2}\right) \quad\left(k_{1}, k_{2} \in K ; x \in G\right) .
$$

LEMMA 17. For any two elements $g, g^{\prime} \in \mathbb{S}$, we can choose a finite number of $g_{i} \in \mathbb{S}$ $(1 \leqslant i \leqslant p)$ with the following property. If $\phi$ is any $C^{\infty} \mu$-spherical function, then

and

$$
\begin{aligned}
& \left|\phi\left(g ; x ; g^{\prime}\right)\right| \leqslant \sum_{1 \leqslant j \leqslant p}\left|\phi\left(x ; g_{j}\right)\right| \\
& \left|\phi\left(g ; x ; g^{\prime}\right)\right| \leqslant \sum_{1 \leqslant j \leqslant p}\left|\phi\left(g_{j} ; x\right)\right|
\end{aligned}
$$

for $x \in G$.

Let $a$ be a maximal abelian subspace of $\mathfrak{p}$. Introduce an order in the space of real linear functions $\alpha$ on $\mathfrak{a}$ and, for any such $\alpha$, let $g_{\alpha}$ denote the subspace of those $X \in \mathfrak{g}$ for which $[H, X]=\alpha(H) X$ for $H \in \mathfrak{a}$. Let $\Sigma$ be the set of all positive roots of $(\mathfrak{g}, \mathfrak{a})$ and $\left(\alpha_{1}, \ldots, \alpha_{l}\right)$ the set of simple roots in $\Sigma$. Put

$$
\mathfrak{n}=\sum_{\alpha>0} g_{\alpha}
$$

Then $\mathfrak{g}=\mathfrak{l}+\mathfrak{a}+\mathfrak{n}$ and $\mathfrak{S}=\mathfrak{A} \mathfrak{A} \mathfrak{R}$ where $\left({ }^{2}\right) \mathfrak{I}=\mathfrak{S}\left(\mathfrak{f}_{c}\right), \mathfrak{A}=\subseteq\left(\mathfrak{a}_{c}\right)$ and $\mathfrak{N}=\subseteq\left(\mathfrak{n}_{c}\right)$.

(1) This means that $V$ is a left $K$-module under $\mu_{1}$ and a right $K$-module under $\mu_{2}$. Moreover, the operations of $K$ on the left, commute with those on the right.

(2) We use here the notation of $[4(\mathrm{~m})$, p. 280]. 
Fix an integer $d \geqslant 0$ such that $g, g^{\prime} \epsilon_{d}(S)$ (see $[4(0), \S 2]$ for the notation). Then we can choose a base $B$ for ${ }_{d}(\mathfrak{S}$ such that every element $b \in B$ has the form $b=x u \nu$ where $x \in \Re$, $u \in \mathfrak{A}, \nu \in \mathfrak{N}$ and

$$
\nu^{a}=\exp \left(\sum_{1 \leqslant i \leqslant l} m_{i} \alpha_{i}(\log a)\right) \nu \quad(a \in A=\exp \mathfrak{a})
$$

$m_{i}$ being nonnegative integers. Then

$$
g^{k}=\sum_{b \in B} a_{b}(k) b, g^{\prime k}=\sum_{b \in B} a_{b}^{\prime}(k) b \quad(k \in K),
$$

where $a_{b}$ and $a_{b}^{\prime}$ are continuous functions on $K$.

Now since any two norms on $V$ are equivalent, we may assume that $\left|\mu_{1}\left(k_{1}\right) v \mu_{2}\left(k_{2}\right)\right|=$ $|v|$ for $k_{1}, k_{2} \in K$ and $v \in V$. Put $A^{+}=\exp \mathfrak{a}^{+}$where $\mathfrak{a}^{+}$is the set of those points $H \in \mathfrak{a}$ where $\alpha(H) \geqslant 0$ for $\alpha \in \Sigma$. Then $G=K A+K$. Put

$$
c=\sup _{k \in \mathbb{K}} \max _{b \in B}\left(\left|a_{b}(k)\right|,\left|a_{b}^{\prime}(k)\right|\right)
$$

Then if $x=k_{1} h k_{2}\left(k_{1}, k_{2} \in K ; h \in A^{+}\right)$, it is clear that

$$
\left|\phi\left(g ; x ; g^{\prime}\right)\right| \leqslant\left|\phi\left(g^{k_{1}-1} ; h ; g^{\prime k_{2}}\right)\right| \leqslant c^{2} \sum_{b, b^{\prime} \in B}\left|\phi\left(b ; h ; b^{\prime}\right)\right| .
$$

Now $b=\varkappa_{b} u_{b} \nu_{b}(b \in B)$ as above. Let us denote the representation of $\Re_{c}$ corresponding to $\mu_{i}$ again by $\mu_{i}(i=1,2)$. For any endomorphism $T$ of $V$, define

$$
|T|=\sup _{|v| \leqslant 1}|T v| \quad(v \in V)
$$

as usual and put

$$
c_{1}=\sup _{b \in B}\left|\mu_{1}\left(\varkappa_{b}\right)\right| \text {. }
$$

Then

$$
\left|\phi\left(b ; h ; b^{\prime}\right)\right|=\left|\mu_{1}\left(\varkappa_{b}\right) \phi\left(h ; u_{b} v_{b}^{{ }^{2-1}} b^{\prime}\right)\right| \leqslant c_{1}\left|\phi\left(h ; u_{b} v_{b} b^{\prime}\right)\right|
$$

since $\alpha_{i}(\log h) \geqslant 0(1 \leqslant i \leqslant l)$. Hence

$$
\left|\phi\left(g ; x ; g^{\prime}\right)\right| \leqslant c^{2} c_{1} \sum_{b, b^{\prime} \in B}\left|\phi\left(h ; u_{b} v_{b} b^{\prime}\right)\right| .
$$

Now let $g_{j}(1 \leqslant j \leqslant p)$ be a base for the subspace of $\left(\mathbb{S}\right.$ spanned by $\left(u_{b} v_{b} b^{\prime}\right)^{k}\left(b, b^{\prime} \in B, k \in K\right)$. Then it is clear that we can choose a number $c_{2} \geqslant 0$ with the following property. If $b, b^{\prime} \in B, k \in K$ and

$$
\left(u_{b} v_{b} b^{\prime}\right)^{k}=\sum_{1 \leqslant j \leqslant p} \gamma_{j} g_{j} \quad\left(\gamma_{j} \in \mathbf{C}\right)
$$

then $\left|\gamma_{j}\right| \leqslant c_{2}$. This shows that 


$$
\left|\phi\left(g ; x ; g^{\prime}\right)\right| \leqslant c_{3} \sum_{1 \leqslant j \leqslant p}\left|\phi\left(x ; g_{j}\right)\right|
$$

for $x \in G$ where $c_{3}=c^{2} c_{1} c_{2}$. Since our hypotheses are symmetrical with respect to left and right, the assertion of the lemma is now obvious.

\section{§ 12. Application to $C_{F}(G)$}

For any finite subset $F$ of $\mathcal{E}_{K}$, define $\alpha_{F}$ as in $\S 6$ and let $\mathcal{C}_{F}(G)$ denote the subspace of all elements in $\mathcal{C}(G)$ of the form $\alpha_{F} * f * \alpha_{F}(f \in \mathcal{C}(G))$. It is clear that an element $f \in \mathcal{C}(G)$ lies in $\mathcal{C}_{F}(G)$ if and only if $\alpha_{F} * f * \alpha_{F}=f$. Hence $C_{F}(G)$ is closed in $\mathcal{C}(G)$.

Put

$$
\begin{gathered}
\nu_{m, g}(f)=\sup (1+\sigma)^{m} \Xi^{-1}|g f|, \\
g \nu_{m}(f)=\sup (1+\sigma)^{m} \Xi^{-1}|\varrho(g) f|
\end{gathered}
$$

for $m \geqslant 0, g \in \mathbb{S}$ and $f \in \mathcal{C}(G)$ and let $S_{1}=\left\{v_{m, g} ; m \geqslant 0, g \in(\mathfrak{S}\}\right.$ and $S_{2}=\left\{v_{m} ; m \geqslant 0, g \in \mathbb{S}\right\}$.

LEMMA 18. Let $F$ be a finite subset of $\mathcal{E}_{K}$. Then each of the three sets of seminorms $S_{1}, S_{2}$ and $S$ define the same topology on $\mathcal{C}_{F}(G)$.

Consider $C(K \times K)$ as a Banach space with the norm

$$
|f|=\sup _{k_{1}, k_{2} \in K}\left|f\left(k_{1}, k_{2}\right)\right| \quad(f \in C(K \times K))
$$

and let $\mu_{1}(k) f$ and $f \mu_{2}(k)(k \in K)$ respectively denote the functions

$$
\left(k_{1}, k_{2}\right) \rightarrow f\left(k^{-1} k_{1}, k_{2}\right) \text { and }\left(k_{1}, k_{2}\right) \rightarrow f\left(k_{1}, k_{2} k^{-1}\right) \quad\left(k_{1}, k_{2} \in K\right) .
$$

Then $\mu=\left(\mu_{1}, \mu_{2}\right)$ is a double representation of $K$ on $C(K \times K)$. Let $C_{p}$ be the subspace of all $f \in C(K \times K)$ such that

$$
f=\int_{K} \alpha_{F}(k) \mu_{1}(k) f d k=\int_{E} \alpha_{F}(k) f \mu_{2}(k) d k .
$$

Then $C_{F}$ is a finite-dimensional space invariant under $\mu$. We denote the restriction of $\mu$ on $C_{F}$ by $\mu_{F}$.

For any $f \in \mathcal{C}_{F}(G)$, define the $\mu_{F}$-spherical function $f^{*}$ from $G$ to $C_{F}$ as follows. If $x \in G$, $f^{*}(x)$ is the function

in $C_{F}$. It is clear that

$$
\left(k_{1}, k_{2}\right) \rightarrow f\left(k_{1}^{-1} x k_{2}^{-1}\right) \quad\left(k_{1}, k_{2} \in K\right)
$$

$$
\left|f^{*}\left(g_{1} ; x ; g_{2}\right)\right|=\sup _{k_{1}, k_{2} \in K}\left|f\left(g_{1}^{k_{1}} ; k_{1} x k_{2}{ }^{-1} ; g_{2}^{k_{2}}\right)\right|
$$

for $g_{1}, g_{2} \in \mathbb{S}$ and $x \in G$. Therefore if we apply Lemma 17 with $V=C_{F}$, Lemma 18 follows immediately. 


\section{\$13. Density of $C_{c}^{\infty}(G)$ in $C(G)$}

Now we come to the proof of Theorem 2. We have to show that $C_{c}{ }^{\infty}(G)$ is dense in $\mathcal{C}(G)$. Fix a finite subset $F$ of $\mathcal{E}_{K}$. In view of Lemma 16, it would be enough to verify the following result.

LFMMA 19. $C_{c}^{\infty}(G) \cap \mathcal{C}_{F}(G)$ is dense in $\mathcal{C}_{F}(G)$.

For any $t>0$, let $G_{t}$ denote the open set consisting of all $x \in G$ with $\sigma(x)<t$. Also let $\xi_{t}$ denote the characteristic function of $G_{t}$. Fix $a>0$ and an element $\alpha \in C_{c}{ }^{\infty}\left(G_{a}\right)$ such that $\alpha\left(k_{1} x k_{2}\right)=\alpha(x)\left(k_{1}, k_{2} \in K ; x \in G\right)$ and

$$
\int_{G} \alpha(x) d x=1
$$

Put

$$
u_{t}=\left(1-\xi_{t}\right) * \alpha=1-\xi_{t} * \alpha,
$$

where the star denotes convolution on $G$ as usual. It is clear thas $u_{t} \in C_{c}{ }^{\infty}(G)$.

LEMMA 20. We have

and

$$
\begin{aligned}
u_{t}(x) & =\left\{\begin{array}{l}
0 \text { if } \sigma(x) \leqslant t-a, \\
1 \text { if } \sigma(x) \geqslant t+a,
\end{array}\right. \\
\left|u_{t}(x ; g)\right| & \leqslant \int_{\bar{G}}|\alpha(y ; g)| d y \quad(x \in G)
\end{aligned}
$$

for $g \in(\mathfrak{S}$.

It is clear that

$$
u_{t}(x)=\int_{G_{a}}\left(1-\xi_{t}\left(x y^{-1}\right)\right) \propto(y) d y
$$

and if we fix $y \in G_{a}$, it follows from Lemma 10 that

$$
\xi_{t}\left(x y^{-1}\right)=\left\{\begin{array}{l}
0 \text { if } \sigma(x) \geqslant t+a \\
1 \text { if } \sigma(x) \leqslant t-a
\end{array}\right.
$$

This gives the first statement of the lemma. Now fix $g \in \mathbb{S}$. Since $g$ is left-invariant, we have

$$
g u_{t}=\left(1-\xi_{t}\right) * g \alpha
$$

Therefore

$$
u_{t}(x ; g)=\int\left(1-\xi_{t}\left(x y^{-1}\right)\right) \alpha(y ; g) d y
$$

and this implies the desired inequality. 
Now fix $f \in \mathcal{C}_{F}(G)$ and put

$$
f_{t}=\left(1-u_{t}\right) f=\left(\xi_{t} * \alpha\right) f .
$$

Since $\alpha$ and $\xi_{t}$ are both invariant under left and right translations of $K$, it is obvious that $f_{t} \in C_{c}{ }^{\infty}(G) \cap C_{F}(G)$. We now claim that $f_{t} \rightarrow f$ in $C_{F}(G)$ as $t \rightarrow+\infty$. Fix $g \in(B)$. Then

$$
g\left(f-f_{t}\right)=g\left(u_{t} f\right)=\sum_{1 \leqslant i \leqslant p} g_{i}^{\prime} u_{t} \cdot g_{i} f
$$

where $g_{i}, g_{i}^{\prime}$ are suitable elements in (S). Moreover,

if $\sigma(x) \geqslant t+a$ and

$$
f(x)-f_{t}(x)=u_{t}(x) f(x)=f(x)
$$

$$
(1+\sigma(x))^{m} \Xi(x)^{-1}|f(x ; g)| \leqslant(1+t)^{-1} v_{m+\mathbf{1} .}(f)
$$

for $\sigma(x) \geqslant t$ and $m \geqslant 0$ in the notation of $\S 12$. Hence

$$
(1+\sigma(x))^{m} \Xi(x)^{-1}\left|f(x ; g)-f_{t}(x ; g)\right| \leqslant(1+t)^{-1} \nu_{m+1 . g}(f)
$$

for $\sigma(x) \geqslant t+a$. Now suppose $\sigma(x)<t+a$. By Lemma $20, f(x)-f_{t}(x)=0$ if $\sigma(x) \leqslant t-a$. So let us assume that $t-a<\sigma(x)<t+a$. Then if $t>a$, we conclude from Lemma 20 that

$$
\begin{aligned}
(1+\sigma(x))^{m} \Xi(x)^{-1}\left|f(x ; g)-f_{t}(x ; g)\right| & \leqslant \sum_{1 \leqslant i \leqslant p} c_{i}(1+\sigma(x))^{m} \Xi(x)^{-1}\left|f\left(x ; g_{i}\right)\right| \\
& \leqslant \sum_{i} c_{i}(1+t-a)^{-1} v_{m+1, g_{i}}(f),
\end{aligned}
$$

where

$$
c_{i}=\int_{G}\left|\alpha\left(y ; g_{i}^{\prime}\right)\right| d y \quad(1 \leqslant i \leqslant p) .
$$

This shows that $v_{m, g}\left(f-f_{t}\right) \rightarrow 0$ as $t \rightarrow+\infty$ and therefore by Lemma 18, $f_{t}$ converges to $f$ in $\mathcal{C}_{F}(G)$. This proves Lemma 19 and therefore also Theorem 2.

\section{§ 14. An inequality}

Let $\mathfrak{h}=\theta(\mathfrak{G})$ be a Cartan subalgebra of $\mathfrak{g}$ and $\mathfrak{m}$ and $\tilde{M}$ the centralizers of $\mathfrak{h} \cap \mathfrak{p}$ in $\mathfrak{g}$ and $G$ respectively. Let $M$ denote the connected component of 1 in $\tilde{M}$.

Fix compatible orders on the spaces of real-valued linear functions on $\mathfrak{h} \cap \mathfrak{f}$ and

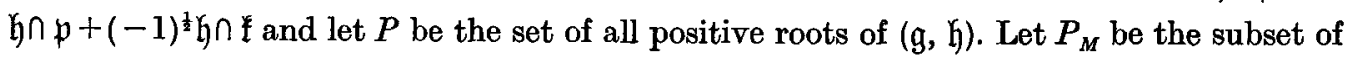
those roots in $P$ which vanish identically on $\mathfrak{h} \cap \mathfrak{p}$ and $P_{+}$the complement of $P_{M}$ in $P$. Put

$$
\mathfrak{H}_{\mathfrak{h}}=\mathfrak{g} \cap\left(\sum_{\alpha \in P_{+}} \mathbf{c} X_{\alpha}\right)
$$


in the notation of $(4(n), \S 4]$. Then $\mathfrak{n}_{\mathfrak{G}}$ is a nilpotent subalgebra of $\mathfrak{g}$ and $\tilde{M}$ normalizes $\mathfrak{n}_{\mathfrak{b}}$. Put

$$
d(m)=\left|\operatorname{det}(\operatorname{Ad}(m))_{\mathfrak{H}_{\mathfrak{h}}}\right|^{\frac{1}{2}} \quad(m \in \tilde{M})
$$

and let $N_{\mathfrak{h}}$ denote the analytic subgroup of $G$ corresponding to $\mathfrak{n}_{\mathfrak{h}}$.

Extend $\mathfrak{h} \cap \mathfrak{p}$ to a maximal abelian subspace $\mathfrak{a}$ of $\mathfrak{p}$ and introduce an order on the dual of $\mathfrak{a}$ which is compatible with that chosen above on the dual of $\mathfrak{h} \cap \mathfrak{p}$. Let $\mathfrak{g}=\mathfrak{f}+\mathfrak{a}+\mathfrak{n}$ be the corresponding Iwasawa decomposition. Then $\mathfrak{n}_{\mathfrak{h}} \subset \mathfrak{n}$. Put

$$
\varrho(H)=\frac{1}{2} \operatorname{tr}(\operatorname{ad} H)_{\mathfrak{n}} \quad(H \in \mathfrak{a})
$$

and define $\mathfrak{a}^{+}$and $A^{+}=\exp \mathfrak{a}^{+}$as usual (see [4 (q), §21]). Then by [4 (j), Theorem 3], we can choose a number $d \geqslant 0$ such that

$$
\sup _{h \in A^{+}}(1+\sigma(h))^{-d} e^{o(\log h)} \Xi(h)<\infty .
$$

Let $\Xi_{M}$ denote the function on $M$ corresponding to $\Xi$ if we replace $(G, K)$ by $(M, K \cap M)$.

Lемма 21. Let dn denote the Haar measure of $N_{\mathfrak{f}}$ and fix $r>r^{\prime} \geqslant 0$. Then we can choose a number $c>0$ such that

$$
d(m) \int_{N_{\mathfrak{h}}}(1+\sigma(m n))^{-(r+2 d)} \Xi(m n) d n \leqslant c(1+\sigma(m))^{-r^{\prime}} \Xi_{M}(m)
$$

for $m \in M$. Moreover, there exists a number $c_{0} \geqslant 1$ such that

$$
1+\sigma(m) \leqslant c_{0}(1+\sigma(m n)) \quad\left(m \in M, n \in N_{\mathfrak{\xi}}\right) .
$$

It is clear that for a fixed $\left.{ }^{1}{ }^{1}\right) m \in M, d n^{m}=d(m)^{2} d n$. Therefore since $m n=n^{m} \cdot m$, we get the following result immediately.

CoR OLLARY. In the above notation, we also have

for $m \in M$.

$$
d(m)^{-1} \int_{N_{\mathfrak{h}}}(1+\sigma(n m))^{-(r+2 d)} \Xi(n m) d n \leqslant c(1+\sigma(m))^{-r^{\prime}} \Xi_{M}(m)
$$

We shall give a proof of Lemma 21 in $\$ 44$.

(1) $y^{x}=x y x^{-1}(x, y \in G)$ as usual. 


\section{\$ 15. The mapping of $\mathrm{C}(G)$ into $C(\tilde{\mathbf{M}})$}

Let us keep to the above notation. Then $M \cap K$ has finite index in $\tilde{M} \cap K$ and therefore $\tilde{M} / M$ is finite. Hence the space $C(\tilde{M})$ is well defined (see $\S 9$ ). Let $\Omega$ be a compact subset of $G$. Then it follows from Lemma 10, [4 (q), Lemma 32] and $\S 14$ that, for any $f \in \mathcal{C}(G)$, the integrals

$$
\int_{N_{\mathfrak{\xi}}}|f(x n)| d n, \int_{N_{\mathfrak{\xi}}}|f(n x)| d n
$$

converge uniformly for $x \in \Omega$ (see also Corollary 2 of Lemma 90). Put

$$
u_{f}(m)=d(m) \int_{N_{\xi}} f(m n) d n=d\left(m^{-1}\right) \int_{N_{9}} f(n m) d n \quad(m \in M),
$$

and ${ }^{(1)} \mathfrak{M}=\subseteq\left(\mathfrak{m}_{c}\right)$. It is easy to verify (see [4 (q), $\left.\S 10\right]$ ) that there exist two automorphisms $\mu \rightarrow \mu^{\prime}$ and $\mu \rightarrow{ }^{\prime} \mu$ of $\mathfrak{M}$ such that

$$
\mu^{\prime}=d^{-1} \mu \circ d,{ }^{\prime} \mu=d \mu \circ d^{-1} \quad(\mu \in \mathfrak{M}) .
$$

Then it follows from what we have said above that $u_{f} \in C^{\infty}(M)$ and

$$
\begin{gathered}
u_{f}\left(\mu_{1:} m\right)=d(m) \int_{N_{\mathfrak{G}}} f\left(\mu_{1}^{\prime} ; m n\right) d n, \\
u_{f}\left(m ; \mu_{2}\right)=d\left(m^{-1}\right) \int f\left(n m ; \mu_{2}\right) d n=d(m) \int f\left(m n ;{ }^{\prime} \mu_{2}\right) d n
\end{gathered}
$$

for $\mu_{1}, \mu_{2} \in \mathfrak{M}$. Since ' $\mu_{2} f \in \mathbb{C}(G)$, we conclude that

$$
u_{f}\left(\mu_{1 ;} ; ; \mu_{2}\right)=d(m) \int f\left(\mu_{1}^{\prime} ; m n ;{ }^{\prime} \mu_{2}\right) d n .
$$

In view of Lemma 21, the following result is now obvious.

LEMMA 22. $f \rightarrow u_{f}$ is a continuous linear mapping of $\mathrm{C}(G)$ into $\mathrm{C}(M)$.

$$
\text { Put } \quad f(x)=\int_{K} f\left(x^{k}\right) d k \quad(x \in G)
$$

for $f \in \mathcal{C}(G)$. Then if follows from Lemma 13 that $f \rightarrow \bar{f}$ is a continuous endomorphism of $\mathcal{C}(G)$. Now define

$$
g_{f}(m)=d(m) \int_{N_{\mathfrak{G}}} f(m n) d n \quad(m \in \tilde{M}) .
$$

(1) We use here the notation of $[4(\mathrm{~m}), \mathrm{p} .280]$. 
THEOREM 3. $f \rightarrow g_{f}$ is a continuous linear mapping of $\mathrm{C}(G)$ into $\mathrm{C}(\tilde{M})$.

This is obvious from Lemma 22.

As usual let 8 denote the center of $\mathfrak{S}$ and $\mu=\mu_{\mathfrak{g} / \mathfrak{m}}$ the homomorphism of [4 (0), $\S 12]$. Since $C_{c}{ }^{\infty}(G)$ is dense in $\mathrm{C}(G)$, we get the following result from the corollary of [4 (q), Lemma 14].

Corollary. Let $z \in \mathbb{Z}$ and $f \in \mathcal{C}(G)$. Then

$$
g_{z f}=\mu(z) g_{f}
$$

\section{\$16. Proof of Theorem 4}

Let $I(G)$ be the space of all continuous functions $f$ on $G$ which are $b i$-invariant under $K$, and $I^{+}(G)$ the subset of all real $f \geqslant 0$. Put $I_{c}{ }^{\infty}(G)=I(G) \cap C_{c}{ }^{\infty}(G)$ and

$$
I_{c}^{+}(G)=C_{c}(G) \cap I^{+}(G)
$$

Let $\cong$ be the centralizer of $\mathfrak{f}$ in $\mathscr{S}$ and $\mu$ a seminorm $\left.{ }^{1}\right)$ on $I_{c}{ }^{\infty}(G)$ satisfying the following two conditions.

1) There exist elements $q_{1}, \ldots, q_{p} \in \mathfrak{Q}$ such that

$$
\mu(f) \leqslant \sum_{1 \leqslant i \leqslant p} \int_{G}\left|q_{i} f\right| \Xi d x \quad\left(f \in I_{c}^{\infty}(G)\right) .
$$

2) If $f_{1}, f_{2}$ are two elements in $I_{c}{ }^{\infty}(G)$ such that $f_{1} \geqslant f_{2} \geqslant 0$, then $\mu\left(f_{1}\right) \geqslant \mu\left(f_{2}\right)$.

For any $\phi \in I^{+}(G)$, put

$$
\mu(\phi)=\sup \mu(f)
$$

where $f$ runs over all functions in $I^{+}(G) \cap I_{c}{ }^{\infty}(G)$ such that $\phi \geqslant f$. Fix a number $r \geqslant 0$ as in Lemma 11.

THEOREM 4. Under the above conditions

$$
\mu\left(\boldsymbol{\Xi}(\mathbf{I}+\sigma)^{-r}\right)<\infty
$$

Fix $\alpha \in I^{+}(G) \cap I_{c}{ }^{\infty}(G)$. Then $\alpha * f \in I_{c}{ }^{\infty}(G)$ for $f \in I_{c}{ }^{+}(G)$ and

$$
\mu(\alpha * f) \leqslant \sum_{1 \leqslant i \leqslant p} \int\left|q_{i} \alpha * f\right| \Xi d x
$$

(1) Here we take the space $I_{c}{ }^{\infty}(G)$ without topology. 
since $q_{i}(\alpha * f)=q_{i}(f * \alpha)=f * q_{i} \alpha=q_{i} \alpha * f$, the convolution being abelian in the present case (see [5 (a), Theorem 4.1, p. 408]). Now

$$
\int\left|q_{i} \alpha * f\right| \Xi d x \leqslant \int\left|\alpha\left(y ; q_{i}\right)\right| f\left(y^{-1} x\right) \Xi(x) d y d x .
$$

Therefore if $V=\operatorname{Supp} \alpha$ and

$$
c_{1}=\sum_{i} \sup \left|q_{i} \alpha\right|
$$

we get

$$
\mu(\alpha * f) \leqslant c_{1} \int_{V \times G} f(x) \Xi(y x) d y d x \text {. }
$$

Since $V$ is compact, we conclude from [4(q), Lemma 32] that there exists a number $c_{2} \geqslant 0$ such that

$$
\mu(\alpha * f) \leqslant c_{2} \int_{G} f \Xi d x
$$

for all $f \in I_{c}{ }^{+}(G)$. It follows without difficulty from condition 2) on $\mu$ that the same inequality continues to hold for $f \in I^{+}(G)$.

Now take $f=\Xi(1+\sigma)^{-r}$. Then $f \in I^{+}(G)$. Moreover we know that $\Xi>0$ everywhere and (see[5 (a), p. 399])

$$
\int_{K} \Xi(x k y) d k=\Xi(x) \Xi(y) \quad(x, y \in G)
$$

Therefore if we choose $\alpha \in I^{+}(G) \cap I_{c}^{\infty}(G)$ such that

$$
\int \alpha \Xi d x=1
$$

it is obvious that $\alpha * \Xi=\Xi$. Then

$$
(\alpha * f)(x)=\int \alpha(y) \Xi\left(y^{-1} x\right)\left(1+\sigma\left(y^{-1} x\right)\right)^{-r} d y
$$

Now

$$
1+\sigma\left(y^{-1} x\right) \leqslant(1+\sigma(y))(1+\sigma(x))
$$

from Lemma 10. Hence

$$
(\alpha * f)(x) \geqslant c_{0}^{-1} f(x)
$$

where

$$
c_{0}=\sup _{y \in V}(1+\sigma(y))^{r}
$$

and $V=\operatorname{Supp} \alpha$ as before. Therefore

$$
\text { Since } \quad \int f \Xi d x=\int \Xi^{2}(1+\sigma)^{-r} d x<\infty,
$$

we get the assertion of the theorem. 


\section{\$ 17. Convergence of certain integrals}

We shall now derive some consequences of Theorem 4. Assume that $G$ is acceptable (see [4 (o), § 18]) and let $A$ be a Cartan subgroup of $G$. We use the notation of [4 (o), $\S 23]$.

THE OR м 5. Fix $r$ as in $\S 16$. Then

$$
\sup _{a \in A^{*}}|\Delta(a)| \int_{G^{*}} \Xi\left(a^{x^{*}}\right)\left(1+\sigma\left(a^{x^{*}}\right)\right)^{-r} d x^{*}<\infty
$$

Let $\mathfrak{h}$ be the Lie algebra of $A$. Put

$$
\mu(f)=\sup _{a \in A^{\prime}}\left|F_{f}(a)\right|, v(f)=\int_{A}\left|\Delta_{M}(a) F_{f}(a)\right| d a \quad\left(f \in I_{c}^{\infty}(G)\right)
$$

in the notation of [4 (o), Theorem 3]. Then it follows from [4 (q), Theorem 4] that there exists a number $c \geqslant 0$ such that

$$
\boldsymbol{v}(f) \leqslant c \int_{G}|f| \Xi d x \quad\left(f \in I_{c}^{\infty}(G)\right) .
$$

Moreover, by [4 (o), Theorem 3], we can select $z_{1}, \ldots, z_{p} \in 3$ such that

$$
\mu(f) \leqslant \sum_{1 \leqslant i \leqslant p} \nu\left(z_{i} f\right) \quad\left(f \in I_{c}^{\infty}(G)\right)
$$

Hence it is obvious that $\mu$ satisfies the two conditions of $\$ 16$. Moreover, it follows from the elementary properties of an integral that if $\phi \in I^{+}(G)$ and $a \in A^{\prime}$, then

$$
\int_{G^{*}} \phi\left(a^{x^{*}}\right) d x^{*}=\sup _{f} f\left(a^{x^{*}}\right) d x^{*}
$$

where $f$ runs over all elements in $I^{+}(G) \cap I_{c}{ }^{\infty}(G)$ such that $f \leqslant \phi$. Therefore the assertion of Theorem 5 is now an immediate consequence of Theorem 4.

Let $\gamma$ be a semisimple element in $G$ and $G_{\gamma}$ the centralizer of $\gamma$ in $G$. Then $G_{\gamma}$ is unimodular and therefore the factor space $\bar{G}=G / G_{\gamma}$ has an invariant measure $d \bar{x}$. Let $x \rightarrow \bar{x}$ denote the projection of $G$ on $\bar{G}$ and put

$$
\gamma^{\bar{x}}=\gamma^{x}=x \gamma x^{-1} \quad(x \in G)
$$

THEOREM 6. $\quad \int_{G / G_{\gamma}} \Xi\left(\gamma^{\bar{x}}\right)\left(1+\sigma\left(\gamma^{\bar{x}}\right)\right)^{-r} d \bar{x}<\infty$.

Let $z$ be the centralizer of $\gamma$ in $\mathfrak{g}$. Since $\gamma$ is semisimple, $z$ is reductive in $\mathfrak{g}$ and rank $z=$ rank $\mathfrak{g}$. Let $\mathfrak{h}$ be a Cartan subalgebra of $z$ which is fundamental in $z$ (see $[4$ (n), 
$\S 11])$ and $A$ the Cartan subgroup of $G$ corresponding to $\mathfrak{h}$. Then $\gamma \in A$. As usual let $P$ and $P_{\hat{z}}$ denote the sets of positive roots of $(\mathfrak{g}, \mathfrak{h})$ and $(\mathfrak{z}, \mathfrak{h})$ respectively and $P_{\mathrm{g} / \mathfrak{z}}$ the complement of $P_{\mathrm{a}}$ in $P$. Put

in the notation of $[4(n), \S 4]$.

$$
\varpi_{z}=\prod_{\alpha \in P_{3}} H_{\alpha}
$$

Lомма 23. (') There exists a number $c \neq 0$ such that

for all $f \in C_{c}^{\infty}(G)$.

$$
F_{f}\left(\gamma ; \varpi_{z}\right)=c \int_{G / G_{\gamma}} f\left(\gamma^{\bar{x}}\right) d \bar{x}
$$

We observe that in view of [4 (o), Lemma 40], the left side has a well-defined meaning: Moreover, since $\gamma$ is semisimple, the orbit $\gamma^{G}$ is closed (see $[1, \S 10.1]$ ) and therefore $[1, \S 5.1]$ the integral on the right is also well defined.

Normalize the invariant measure $d y^{*}$ on $G_{\gamma} / A_{0}$ in such a way that $d x^{*}=d \bar{x} d y^{*}$. Let $U$ be an open, connected neighborhood of 1 in $A$ such that $\operatorname{det}(\operatorname{Ad}(a)-1)_{\mathfrak{g} / z} \neq 0$ for $a \in U$. Put $U^{\prime}=U \cap\left(\gamma^{-1} A^{\prime}\right)$. Then an element $a \in U$ lies in $U^{\prime}$ if and only if $\operatorname{det}(\operatorname{Ad}(\gamma a)-1)_{3 / \hbar} \neq 0$. Moreover, we may assume (see [4 (i), Theorem 1]) that $U$ has the following property. For any compact set $\Omega$ in $G$, there exists a compact subset $C$ of $\bar{G}$ such that $x U x^{-1} \cap \Omega=\emptyset(x \in G)$ unless $\bar{x} \in C$.

Fix $f \in C_{c}^{\infty}(G)$ and select $C$ as above corresponding to $\Omega=\operatorname{Supp} f$. Then if $a \in U^{\prime}$,

$$
\int_{G^{*}} f\left((\gamma a)^{x^{*}}\right) d x^{*}=\int_{C} d \bar{x} \int_{G_{\gamma^{*}}} f\left(x(\gamma a)^{y^{*}} x^{-1}\right) d y^{*} .
$$

Let $G_{\gamma}{ }^{0}$ denote the connected component of 1 in $G_{\gamma}$ and $Z$ the center of $G$. Then $Z G_{\gamma}{ }^{0}$ has finite index in $G_{\gamma}$ (see [4(h), Lemma 15]). Let $N$ denote this index and choose $y_{i}(1 \leqslant i \leqslant N)$ in $G_{\gamma}$ such that

$$
G_{\gamma}=\bigcup_{1 \leqslant i \leqslant N} y_{i} Z G_{\gamma}^{0}
$$

Define

$$
g_{x}(y)=\sum_{1 \leqslant i \leqslant N} f\left(x \gamma y_{i} y y_{i}^{-1} x^{-1}\right) \quad\left(y \in G_{\gamma}{ }^{0}\right)
$$

for $x \in G$. Then it is clear that

for $a \in U^{\prime}$.

$$
\int_{G^{*}} f\left((\gamma a)^{x^{*}}\right) d x^{*}=\int_{C} d \bar{x} \int_{\left(G_{\gamma}{ }^{0}\right)} g_{x}\left(a^{y *}\right) d y^{*}
$$

Choose an open and convex neighborhood $V$ of zero in $\mathfrak{h}$ such that $\exp V \subset U$ and $|\alpha(H)|<1$ for $\alpha \in P$ and $H \in V$. Let $V^{\prime}$ denote the set of all points $H \in V$ where

(1) Cf. Langlands [6, p. 114].

3-662900. Acta mathematica. 116. Imprimé le 10 juin 1966. 
Then $\exp V^{\prime} \subset U^{\prime}$ and

$$
\pi_{\mathfrak{z}}(H)=\prod_{\alpha \in P_{\mathfrak{z}}} \alpha(H) \neq 0
$$

$$
\Delta(\gamma \exp H)=\xi_{\underline{Q}}(\gamma) \prod_{\alpha \in P_{g / 3}}\left(e^{\alpha(H) / 2}-\xi_{\alpha}(\gamma)^{-1} e^{-\alpha(H) / 2}\right) \Delta_{3}(H)
$$

for $H \in V$. Here

$$
\Delta_{\mathrm{z}}(H)=\prod_{\alpha \in P_{\mathrm{z}}}\left(e^{\alpha(H) / 2}-e^{-\alpha(H) / 2}\right) .
$$

Let $D$ denote the differential operator on $\mathfrak{h}$ given by

$$
D=\xi_{\varrho}(\gamma) \partial\left(\varpi_{z}\right) \circ \prod_{\alpha \in P_{\mathfrak{g} / \mathfrak{z}}}\left(e^{\alpha / 2}-\xi_{\alpha}(\gamma)^{-1} e^{-\alpha / 2}\right) .
$$

As usual $D_{0}$ denotes the local expression of $D$ at the origin (see $[4$ (f), p. 90]).

LEM M 24.

$$
D_{0}=\xi_{Q}(\gamma) \prod_{\alpha \in P_{g / z}}\left(1-\xi_{\alpha}(\gamma)^{-1}\right) \partial\left(\varpi_{\gamma}\right)
$$

Fix $q \in S\left(\mathfrak{h}_{c}\right)$ such that

$$
\partial(q)=D_{0}-\xi_{e}(\gamma) \prod_{\alpha \in P_{g} / z}\left(1-\xi_{\alpha}(\gamma)^{-1}\right) \partial\left(w_{z}\right)
$$

If $p$ is the number of roots in $P_{z}$, it is clear that $d^{0} \varpi_{z}=p$ and $d^{0} q<p$. On the other hand, it is easy to see that $D_{0}^{s_{\alpha}}=-D_{0}$ for any $\alpha \in P_{z}$. Therefore $\varpi_{z}$ divides $q$ in $S\left(\mathfrak{h}_{c}\right)$ (see [4 (f), Lemma 10]) and this shows that $q=0$.

For any function $g \in C_{c}{ }^{\infty}\left(G_{\gamma}{ }^{0}\right)$, define

$$
\phi_{g}(H)=\Delta_{z}(H) \int_{\left(G_{\gamma}{ }^{*}\right)^{*}} f\left((\exp H)^{y^{*}}\right) d y^{*} \quad\left(H \in V^{\prime}\right) .
$$

Then by [4 (n) Theorem 3], and [4 (i), Lemma 19], there exists a number $c_{0} \neq 0$ such that

$$
\lim _{H \rightarrow 0} \phi_{o}\left(H ; \partial\left(\varpi_{\jmath}\right)\right)=c_{0} g(1) \quad\left(H \in V^{\prime}\right)
$$

for every $g \in C_{c}^{\infty}\left(G_{\gamma}{ }^{0}\right)$. Hence it follows from Lemma 24 that

$$
F_{f}\left(\gamma ; w_{z}\right)=c_{1} \int_{C} g_{x}(1) d \bar{x}=c_{1} N \int_{G / G_{y}} f\left(\gamma^{\bar{x}}\right) d \bar{x},
$$

where

This proves Lemma 23.

$$
c_{1}=c_{0} \varepsilon_{R}(\gamma) \xi_{\varrho}(\gamma) \prod_{\alpha \in P_{g / \delta}}\left(1-\xi_{\alpha}(\gamma)^{-1}\right) .
$$

Now we come to the proof of Theorem 6. Put 


$$
\mu(f)=\left|\int_{G / G_{\gamma}} f\left(\gamma^{\bar{x}}\right) d \bar{x}\right|=\left|c^{-1} F_{f}\left(\gamma ; \varpi_{\bar{z}}\right)\right| \quad\left(f \in I_{c}^{\infty}(G)\right)
$$

and define $\nu(f)$ as in the proof of Theorem 5. By [4 (o), Theorem 3], we can choose $z_{1}, \ldots, z_{p} \in 3$ such that

$$
\mu(f) \leqslant \sum_{1 \leqslant i \leqslant p} v\left(z_{i} f\right) \quad\left(f \in I_{c}^{\infty}(G)\right)
$$

This shows (see the proof of Theorem 5 ) that $\mu$ fulfills the two conditions of $\$ 16$. Moreover, it is clear that

$$
\int_{G / G_{\gamma}} \phi\left(\gamma^{\bar{x}}\right) d \bar{x}=\sup _{f} \int_{G / G_{\gamma}} f\left(\gamma^{\bar{x}}\right) d \bar{x} \quad\left(\phi \in I^{+}(G)\right),
$$

where $f$ runs over all functions in $I^{+}(G) \cap I_{c}^{\infty}(G)$ such that $f \leqslant \phi$. Therefore Theorem 6 follows from Theorem 4.

\section{\$18. The mapping $f \rightarrow F_{f}$}

We return to the notation of $\S 15$ and define the function $D_{\mathfrak{h}}$ on $G$ as in [4 (q), Lemma 35]. Also we recall that $S$ is the set of all continuous seminorms on $C(G)$.

LEMMA 25. Put

Then $v_{1} \in S$.

$$
\nu_{1}(f)=\int_{G}|f|\left|D_{\mathfrak{h}}\right|^{-\frac{1}{2}} d x \quad(f \in \mathcal{C}(G))
$$

Fix $r$ as in Lemma 11 and put

Then it is clear that

$$
\nu(f)=\sup |f| \Xi^{-1}(1+\sigma)^{r} \quad(f \in C(G)) .
$$

$$
\nu_{1}(f) \leqslant v(f) \int_{G} \Xi(1+\sigma)^{-r}\left|D_{\mathrm{h}}\right|^{-\frac{1}{2}} d x
$$

and therefore our assertion follows from Lemma 11 and [4 (q), Lemma 35].

Now assume that $G$ is acceptable and let $A=A_{\mathfrak{b}}$ be the Cartan subgroup of $G$ corresponding to $\mathfrak{h}$. Since $K$ is compact, $A$ has only a finite number of connected components. Define $A^{\prime}(I)$ as in [4(o), $\left.\S 22\right]$. Then the space $C\left(A^{\prime}(I)\right)$ is well defined (see $\S 9$ ). For any $f \in C_{c}^{\infty}(G)$, define the function $F_{f} \in C^{\infty}\left(A^{\prime}(I)\right)$ as in [4(0), §22].

LEMMA 26. Let $S\left(A^{\prime}(I)\right)$ be the set of all continuous seminorms on $\mathrm{C}\left(A^{\prime}(I)\right)$. Then $F_{f} \in \mathrm{C}\left(A^{\prime}(I)\right)$ and for a given $\nu_{0} \in S\left(A^{\prime}(I)\right)$, we can choose $\nu \in S$ such that

$$
\nu_{0}\left(F_{f}\right) \leqslant v(f) \quad\left(f \in C_{c}^{\infty}(G)\right) .
$$


We use induction on $\operatorname{dim} \mathfrak{g}$. Let $\mathfrak{c}$ be the center of $\mathfrak{g}$ and first assume that $\mathfrak{h} \cap \mathfrak{p} \not \mathfrak{c}$. Then $\operatorname{dim} \mathfrak{m}<\operatorname{dim} \mathfrak{g}$ and the induction hypothesis is applicable to $M$ and therefore our assertion follows immediately from Theorem 3 and [4 (o), Lemma 52] (see also [4 (q), § 10]). Hence we may suppose that $\mathfrak{h} \cap \mathfrak{p}=\mathfrak{c}_{\mathfrak{p}}$ where $\mathfrak{c}_{\mathfrak{p}}=\mathfrak{c} \cap \mathfrak{p}$ as before. Let us assume further that $\mathfrak{c}_{\mathfrak{p}} \neq\{0\}$ and put $\mathfrak{g}_{\mathfrak{1}}=\mathfrak{f}+[\mathfrak{f}, \mathfrak{p}]$. Then $\mathfrak{g}$ is the direct sum of $\mathfrak{c}_{\mathfrak{p}}$ and $\mathfrak{g}_{1}$ and $G$ is the direct product of the corresponding subgroups $C_{\mathfrak{p}}$ and $G_{1}$. Put $\left({ }^{1}\right) \mathfrak{S}_{\mathfrak{p}}=\mathfrak{S}_{\left(\mathfrak{c}_{\mathfrak{c}}\right)}$ and $\mathfrak{G}_{1}=\mathfrak{S}\left(\mathfrak{g}_{1 c}\right)$. Then we may assume, without loss of generality, that

$$
v_{0}(g)=\sup _{h \in A^{\prime}(I)}(1+\sigma(h))^{r}|g(h ; \gamma u)| \quad\left(g \in \mathcal{C}\left(A^{\prime}(I)\right)\right)
$$

for some $r \geqslant 0, \gamma \in \mathfrak{E}_{\mathfrak{p}}$ and $u \in \mathfrak{S}\left(\mathfrak{h}_{1 c}\right)$ where $\mathfrak{h}_{1}=\mathfrak{h} \cap \mathfrak{g}_{1}$. For any $f \in \mathcal{C}(G)$ and $c \in C_{\mathfrak{p}}$, let $f_{c}$ denote the function $x \rightarrow f(c x ; \gamma)\left(x \in G_{1}\right)$ on $G_{1}$. Put $A_{1}{ }^{\prime}(I)=G_{1} \cap A^{\prime}(I)$ and let $F_{g}\left(g \in C_{c}{ }^{\infty}\left(G_{1}\right)\right)$ denote the function on $A_{1}^{\prime}(I)$ corresponding to [4(o), $\left.\$ 22\right]$. Then it is obvious that

$$
(1+\sigma(c h))^{r}\left|F_{j}(c h ; \gamma u)\right| \leqslant(1+\sigma(c))^{r}(1+\sigma(h))^{r}\left|F_{f_{c}}(h ; u)\right|
$$

for $c \in C_{\mathfrak{p}}$ and $h \in A_{1}{ }^{\prime}(I)$. Since $\operatorname{dim} \mathfrak{g}_{1}<\operatorname{dim} \mathrm{g}$, we can, by induction hypothesis, choose a continuous seminorm $v_{1}$ on $\mathrm{C}\left(G_{1}\right)$ such that

$$
(1+\sigma(h))^{r}\left|F_{g}(h ; u)\right| \leqslant \nu_{1}(g)
$$

for $g \in C_{c}{ }^{\infty}\left(G_{1}\right)$ and $h \in A_{1}{ }^{\prime}(I)$. Then it follows that

$$
\nu_{0}\left(F_{f}\right) \leqslant \sup _{c \in C_{\mathfrak{p}}}(1+\sigma(c))^{r} \nu_{1}\left(f_{c}\right) \quad\left(f \in C_{c}^{\infty}(G)\right) .
$$

Now put

$$
v(f)=\sup _{c \in C_{\mathfrak{p}}}(1+\sigma(c))^{\gamma} v_{1}\left(f_{c}\right) \quad(f \in C(G))
$$

Since $\Xi(c x)=\Xi(x)$ and $\sigma(c x) \geqslant \max (\sigma(c), \sigma(x))$ (see $\S 7$ ) for $c \in C_{\mathfrak{y}}$ and $x \in G_{1}$, it is easy to verify that $\nu \in S$.

So now we may suppose that $\mathfrak{c}_{\mathfrak{p}}=\mathfrak{h} \cap \mathfrak{p}=\{0\}$ and therefore $\mathfrak{h} \subset \mathfrak{l}$. Put

$$
\nu_{\mathbf{1}}(f)=\int_{G}|f|\left|D_{\mathfrak{h}}\right|^{-\frac{1}{2}} d x \quad(f \in C(G)) .
$$

Then $\nu_{1} \in S$ by Lemma 25. Therefore since $A \subset K$ in the present case, our assertion follows from [4 (o), Theorem 3]. This completes the proof of Lemma 26.

Since $C_{c}{ }^{\infty}(G)$ is dense in $\mathrm{C}(G)$ (Theorem 2) and $\mathrm{C}\left(A^{\prime}(I)\right.$ ) is complete, it is clear that that $f \rightarrow F_{f}$ can be extended uniquely to a continuous mapping of $\mathrm{C}(G)$ into $\mathrm{C}\left(A^{\prime}(I)\right)$. Thus, for every $f \in \mathcal{C}(G)$, we get a function $F_{f} \in \mathcal{C}\left(A^{\prime}(I)\right)$.

(1) We use here the notation of $[4(\mathrm{~m})$, p. 280]. 
Lемма 27. Let $f \in \mathrm{C}(G)$. Then

$$
F_{f}(a)=\varepsilon_{R}(a) \Delta(a) \int_{G^{*}} f\left(a^{x^{*}}\right) d x^{*} \quad\left(a \in A^{\prime}\right)
$$

in the notation of $[4(\mathrm{o}), \S 23]$.

It is obvious from Theorem 5 that the integral on the right is well defined. Now choose a sequence $f_{j} \in C_{c}{ }^{\infty}(G)(j \geqslant 1)$ such that $f_{j} \rightarrow f$ in $\mathcal{C}(G)$ and put $\phi_{j}=f-f_{j}$. Then, in view of the definition of $F_{f}$, it would be enough to verify that

$$
\sup _{A^{\prime}}|\Delta(a)| \int_{G^{*}}\left|\phi_{j}\left(a^{x^{*}}\right)\right| d x^{*} \rightarrow 0 .
$$

But since $\phi_{j} \rightarrow 0$ in $C(G)$, this is obvious from Theorem 5 .

Let $B$ be another Cartan subgroup of $G$ conjugate to $A$. Fix $x \in G$ such that $B=A^{x}$. Then the isomorphism $a \rightarrow a^{x}$ defines a linear bijection of $\mathcal{C}\left(A^{\prime}(I)\right)$ on a subspace $\mathcal{C}\left(B^{\prime}(I)\right)$ of $C^{\infty}\left(B^{\prime}(I)\right)$. We topologize $\mathrm{C}\left(B^{\prime}(I)\right)$ so as to make this bijection a homeomorphism. It is easy to verify that this topology is independent of the choice of $x$.

Now let us drop the condition that $\mathfrak{h}=\theta(\mathfrak{h})$ and define

$$
F_{f}(a)=\varepsilon_{R}(a) \Delta(a) \int_{G^{*}} f\left(a^{x^{*}}\right) d x^{*} \quad\left(f \in \mathcal{C}(G), a \in A^{\prime}(I)\right) .
$$

It follows from Theorem 5 that this integral exists. Since $\mathfrak{h}$ is conjugate to some Cartan subalgebra which is stable under $\theta$, it is obvious from Lemmas 26 and 27 that $f \rightarrow F_{f}$ is a continuous mapping of $\mathrm{C}(G)$ into $\mathrm{C}\left(A^{\prime}(I)\right)$.

$$
\text { Lемма 28. } \quad F_{f}\left(\gamma ; \varpi_{z}\right)=c \int_{G / G_{\gamma}} f\left(\gamma^{\bar{x}}\right) d \bar{x}
$$

for $f \in \mathcal{C}(G)$ in the notation of Lemma 23.

It follows from Theorem 6 that the integral on the right is well defined. The rest of the argument is similar to that given above for Lemma 27.

Let us now return to the notation of $\S 15$. If we replace $G$ by $M$, we get the corresponding mapping $g \rightarrow F_{g}{ }^{M}$ of $\mathcal{C}(M)$ into $\mathcal{C}\left(A_{0}{ }^{\prime}(I)\right)$ where $A_{0}=A \cap M$ and $A_{0}{ }^{\prime}(I)=A_{0} \cap A^{\prime}(I)$. Define $Z_{A}=Z(A)$ as in [4 (q), $\left.\S 12\right]$. Then the following result is obvious from [4 (o), Lemma 52] (see also [4 (q), § 10]), Theorem 3 and Lemma 26.

LEMмA 29. For any $a \in Z_{A}$, put

$$
g_{f, a}(m)=g_{f}(a m) \quad(m \in M, f \in \mathcal{C}(G))
$$


in the notation of Theorem 3. Then there exists a number $c>0$ such that

for $h \in A_{0}^{\prime}(I)$.

$$
F_{f}(a h)=c \xi_{Q}(a) F_{\sigma_{f, a}}{ }^{M}(h) \quad\left(a \in Z_{A}, f \in \mathcal{C}(G)\right)
$$

\section{\$ 19. A criterion for an invariant eigendistribution to be tempered}

Let $\Theta$ be a distribution on $\mathrm{G}$. Then $\Theta$ is said to be 3 -finite, if the space of all distributions of the form $z \Theta(z \in \mathbb{Z})$ has finite dimension. We recall (see [4 (o), Theorem 2]) that an invariant and 8 -finite distribution is actually a locally summable function which is analytic on the regular set $G^{\prime}$.

Define $D$ as in $[4(0), \S 28]$ and let $A_{i}(1 \leqslant i \leqslant r)$ be a complete set of Cartan subgroups of $G$, no two of which are conjugate in $G$. As usual put $A_{i}^{\prime}=A_{i} \cap G^{\prime}$.

THE if and only if there exists a number $s \geqslant 0$ such that

$$
\sup _{a \in A_{i}^{\prime}}(1+\sigma(a))^{-s}|D(a)|^{\frac{k}{2}}|\Theta(a)|<\infty \quad(1 \leqslant i \leqslant r) .
$$

Let $\mathfrak{a}_{i}$ be the Lie algebra of $A_{i}$. In view of Lemma 10, we can obviously assume that $\theta\left(\mathfrak{a}_{i}\right)=\mathfrak{a}_{i}$. Let us now use the notation of $[4(\mathrm{p})$, Lemma 63]. Then

$$
\int_{G} f d x=\sum_{1 \leqslant i \leqslant r} c_{i} \int_{A_{i}} \varepsilon_{i, R} \operatorname{conj} \Delta_{i} \cdot F_{f, i} d_{i} a \quad\left(f \in C_{c}^{\infty}(G)\right)
$$

where $F_{f, i}=F_{f}$ and $\varepsilon_{i, R}=\varepsilon_{R}$ for $A=A_{i}$ (see $[4(0), \S 22]$ ). Now choose $c \geqslant 0$ such that

$$
|D(a)|^{\frac{1}{2}}|\Theta(a)| \leqslant c(1+\sigma(a))^{s} \quad\left(a \in A_{i}^{\prime}, 1 \leqslant i \leqslant r\right) .
$$

Since $|D(a)|=\left|\Delta_{i}(a)\right|^{2}\left(a \in A_{i}\right)$, it is clear that

$$
|\Theta(f)|=\left|\int_{G} \Theta f d x\right| \leqslant c \sum_{1 \leqslant i \leqslant r} c_{i} \int_{A_{i}}(1+\sigma(a))^{s}\left|F_{f, i}(a)\right| d_{i} a
$$

for $f \in C_{c}{ }^{\infty}(G)$. Hence it follows immediately from Lemma 26, that $\Theta$ is tempered.

Before proving the converse, we shall derive the following consequence of the theorem.

CoROLLARY. Suppose $\Theta$ is tempered. Then, in the above notation,

$$
\Theta(f)=\sum_{1 \leqslant i \leqslant r} c_{i} \int_{A_{i}} \varepsilon_{i, R} \Phi_{i} F_{f, l} d_{i} a \quad(f \in \mathcal{C}(G))
$$


where

$$
\Phi_{i}(a)=\Theta(a) \cdot \operatorname{conj} \Delta_{i}(a) \quad\left(a \in A_{i}^{\prime}\right) .
$$

Fix $f \in C(G)$ and choose a variable element $\alpha \in C_{c}{ }^{\infty}(G)$, which converges to $f$ in $C(G)$. Then

But

$$
\Theta(f)=\lim _{\alpha \rightarrow f} \Theta(\alpha)
$$

$$
\Theta(\alpha)=\int \Theta \alpha d x=\sum_{1 \leqslant i \leqslant r} c_{i} \int_{A_{i}} \varepsilon_{i, R} \Phi_{i} F_{\alpha, i} d_{i} a
$$

and so our assertion is an immediate consequence of Lemma 26 and the above theorem.

Now in order to prove the second part of Theorem 7, we need some preparation. Define $S$ and $S_{1}$ as in Lemma 18 .

LFMм 30. Let $\Theta$ be a tempered and invariant distribution on $G$. Then $\Theta$ is continuous in the topology defined on $\mathrm{C}(G)$ by $S_{1}$.

Fix a function $\alpha \in C_{c}^{\infty}(G)$, such that $\int \alpha d x=1$ and, for any $f \in C_{c}^{\infty}(G)$, put

$$
f_{0}(x)=\int_{G} \alpha(y) f\left(x^{y}\right) d y \quad(x \in G) .
$$

Then

$$
f_{0}\left(z_{1} x z_{2}\right)=\int_{G} \alpha\left(y z_{1}^{-1}\right) f\left(y x z_{2} z_{1} y^{-1}\right) d y \quad\left(z_{1}, z_{2} \in G\right) .
$$

Fix $g^{\prime}, g^{\prime \prime} \in \mathbb{S}$. Then it is clear that we can select $g_{1}, \ldots, g_{p} \in \mathbb{S}$ such that

$$
\left|f_{0}\left(g^{\prime} ; x ; g^{\prime \prime}\right)\right| \leqslant \sum_{1 \leqslant i \leqslant p} \sup _{y \in \Omega}\left|f\left(y x y^{-1} ; g_{i}\right)\right| \quad(x \in G)
$$

for all $f \in C_{c}^{\infty}(G)$. Here $\Omega=\operatorname{Supp} \alpha$. Now fix $m \geqslant 0$. Then by Lemma 10 and $[4$ (q), Lemma 32], we can choose $c \geqslant 0$ such that

$$
\sup _{G}\left|f_{0}\left(g^{\prime} ; x ; g^{\prime \prime}\right)\right| \Xi(x)^{-1}(1+\sigma(x))^{m} \leqslant c \sum_{1 \leqslant i \leqslant p} \sup _{G}\left|f\left(x ; g_{i}\right)\right| \Xi(x)^{-1}(1+\sigma(x))^{m} .
$$

This shows that, for a given $\nu \in S$, there exists a number $c \geqslant 0$ and a finite set $\left(v_{1}, \ldots, v_{p}\right)$ of elements in $S_{1}$, such that

$$
\nu\left(f_{0}\right) \leqslant c \sum_{1 \leqslant i \leqslant p} \nu_{i}(f) \quad\left(f \in C_{c}^{\infty}(G)\right)
$$

On the other hand, since $\Theta$ is tempered, we can choose $\nu \in S$ such that

$$
|\Theta(f)| \leqslant v(f) \quad\left(f \in C_{c}^{\infty}(G)\right) .
$$

Moreover,

$$
\Theta(f)=\Theta\left(f_{0}\right)
$$

since $\Theta$ is invariant. Hence 


$$
|\Theta(f)|=\left|\Theta\left(f_{0}\right)\right| \leqslant v\left(f_{0}\right) \leqslant c \sum_{1 \leqslant i \leqslant p} v_{i}(f) \quad\left(f \in C_{c}^{\infty}(G)\right)
$$

and this proves our assertion.

Now fix a Cartan subalgebra $\mathfrak{h}=\theta(\mathfrak{h})$ of $\mathfrak{g}$ and let $A$ be the corresponding Cartan subgroup of $G$. Let $A_{0}$ be the center of $A$ and $\tilde{A}$ the normalizer of $A$ in $G$. Put $W_{A}=\tilde{A} / A_{0}$ and let $x \rightarrow x^{*}$ denote the natural projection of $G$ on $G^{*}=G / A_{0}$. Then $W_{A}$ is a finite group and $a^{s}$ and $x^{*} s\left(s \in W_{A}, a \in A, x^{*} \in G^{*}\right)$ are defined as usual (see [4(o), $\left.\left.\S 20\right]\right)$. If $\beta$ is any function on $A^{\prime}$, we denote by $\beta^{s}$ the function $a \rightarrow \beta\left(a^{s^{-1}}\right)\left(a \in A^{\prime}, s \in W_{A}\right)$.

Put $G_{A}=\left(A^{\prime}\right)^{G}$ as usual and normalize the measures $d x$, $d a$ and $d x^{*}$ in accordance with Lemma 91. Fix a function $\alpha^{*} \in C_{c}{ }^{\infty}\left(G^{*}\right)$ such that $\alpha^{*}\left(x^{*} s\right)=\alpha^{*}\left(x^{*}\right)\left(x^{*} \in G^{*}, s \in W_{A}\right)$ and

$$
\int_{G^{*}} \alpha^{*} d x^{*}=1
$$

Then, for any $\beta \in C_{c}^{\infty}\left(A^{\prime}\right)$, we define $f_{\beta} \in C_{c}^{\infty}\left(G_{A}\right)$ as follows.

$$
f_{\beta}\left(a^{x}\right)=\alpha^{*}\left(x^{*}\right) \Delta(a)^{-1} \sum_{s \in \mathbb{W}_{\Delta}} \varepsilon(s) \beta\left(a^{s}\right) \quad\left(a \in A^{\prime}, x \in G\right) .
$$

Here $\varepsilon(s)$ has the usual meaning so that $\Delta^{s}=\varepsilon(s) \Delta$. Then it follows from Lemma 9 I that

$$
\Theta\left(f_{\beta}\right)=\int_{A} \Phi \beta d a \quad\left(\beta \in C_{c}^{\infty}\left(A^{\prime}\right)\right),
$$

where $\Phi(a)=\Theta(a) \cdot \operatorname{conj} \Delta(a)\left(a \in A^{\prime}\right)$.

Let $Q$ be the set of all roots of $(\mathfrak{g}, \mathfrak{h})$. Then for each $\alpha \in Q$, we have the character $\xi_{\alpha}$ of $A$ (see [4 (o), $\S 18]$ ). Let $\eta_{\alpha}$ denote the function $\left(1-\xi_{\alpha}{ }^{-1}\right)^{-1}$ on $A^{\prime}$ and let $R$ be the ring of analytic functions on $A^{\prime}$ generated over $\mathbf{C}$ by 1 and $\eta_{\alpha}(\alpha \in Q)$. It is obvious that $R$ is stable under $W_{A}$. Moreover, one finds directly by differentiation that

$$
H \eta_{\alpha}=\alpha(H) \eta_{\alpha}\left(1-\eta_{\alpha}\right) \quad(\alpha \in Q, H \in \mathfrak{h})
$$

This shows that $R$ is also stable under the differential operators in $\subseteq\left(\mathfrak{h}_{c}\right)$.

Fix a connected component $A^{+}$of $A^{\prime}$. Since $K$ is compact, $A^{\prime}$ has only a finite number of connected components (see [4 (e), Lemma 9]) and so, in order to complete.the proof of Theorem 7 , it would be enough to show that there exist numbers $c, m \geqslant 0$ such that

$$
|\Phi(a)| \leqslant c(1+\sigma(a))^{m} \quad\left(a \in A^{+}\right)
$$

Put $\mathfrak{h}_{1}=\mathfrak{h} \cap \mathfrak{f}, \mathfrak{h}_{2}=\mathfrak{h} \cap \mathfrak{p}, A_{1}=A \cap K, A_{1}{ }^{0}=\operatorname{exp~} \mathfrak{h}_{1}$ and $A_{2}=\exp \mathfrak{h}_{2}$. Then $A_{1}{ }^{0}$ is the connected component of 1 in $A_{1}$. For any $a \in A$, let $a_{i}$ denote the component of $a$ in $A_{i}(i=1,2)$ so that $a=a_{1} a_{2}$. Let $\mathfrak{h}_{2}^{\prime}$ be the set of all $H \in \mathfrak{h}_{2}$ such that $\alpha(H) \neq 0$ for any root $\alpha$ of $(\mathfrak{g}, \mathfrak{h})$ which 
is not identically zero on $\mathfrak{h}_{2}$. Then $\mathfrak{h}_{2}{ }^{\prime}$ has only a finite number of connected components. Fix a connected component $\mathfrak{h}_{2}{ }^{+}$of $\mathfrak{h}_{2}{ }^{\prime}$ such that $A^{+}$meets $A_{1} A_{2}{ }^{+}$. (Here $A_{2}{ }^{+}=\exp \mathfrak{h}_{2}{ }^{+}$.) It would be sufficient to prove the required inequality for $a \in A^{++}=A^{+} \cap\left(A_{1} A_{2}{ }^{+}\right)$.

Fix an element $b \exp H_{0}\left(b \in A_{1}, H_{0} \in \mathfrak{h}_{2}{ }^{+}\right)$in $A^{++}$and let $z$ denote the centralizer of $b A_{1}{ }^{0}$ in $g$. Let $Q_{\mathfrak{z}}$ be the set of all roots of $(\mathfrak{z}, \mathfrak{h})$. Then every root in $Q_{\mathfrak{z}}$ is real (see [4(n), $\S 4]$ ) and $\alpha\left(H_{0}\right) \neq 0$ for $\alpha \in Q_{3}$.

Let $\mathfrak{F}$ and $\mathfrak{F}_{2}$ be the spaces of real linear functions on $(-1)^{\frac{1}{2}} \mathfrak{h}_{1}+\mathfrak{h}_{2}$ and $\mathfrak{h}_{2}$ respectively. Introduce compatible orders in $\mathfrak{F}_{\text {and }} \mathfrak{F}_{2}$ such that an element $\lambda \in \mathfrak{F}_{2}$ is positive whenever $\lambda\left(H_{0}\right)>0$. Let $P$ be the set of all positive roots of $(\mathfrak{g}, \mathfrak{h})$ under this order and put $P_{\mathfrak{z}}=P \cap Q_{\mathfrak{z}}$. We may use this order for the definition of $\Delta$ and $\xi_{Q}$ (see [4 (o), § 19]). Then

where $\eta=\prod_{\alpha \in P} \eta_{\alpha} \in R$.

$$
\Delta^{-1}=\xi_{\varrho}^{-1} \eta
$$

Fix a compact set $C=C^{-1}$ in $G$ such that Supp $\alpha^{*} \subset C^{*}$.

LEMмA 31. For any $g \in(S)$, we can select $u_{i} \in \mathfrak{S}\left(\mathfrak{h}_{c}\right)$ and $\eta_{i} \in R(1 \leqslant i \leqslant p)$ such that

$$
\left|f_{\beta}\left(a^{x} ; g\right)\right| \leqslant\left|\xi_{\varrho}(a)\right|^{-1} \sum_{1 \leqslant i \leqslant p} \sum_{s \in W_{\mathcal{A}}}\left|\eta_{i}(a) \beta^{s}\left(a ; u_{i}\right)\right|
$$

for $\beta \in C_{c}^{\infty}\left(A^{\prime}\right), a \in A^{\prime}$ and $x \in C$.

Assuming this lemma, we shall first finish the proof of Theorem 7. By Lemma 10 and [4 (q), Lemma 32], we can choose $c \geqslant 1$ such that

$$
\Xi\left(y^{x}\right) \leqslant c \Xi(y), 1+\sigma\left(y^{x}\right) \leqslant c(1+\sigma(y)) \quad(x \in C, y \in G) .
$$

Since $C=C^{-1}$, this implies that

$$
\begin{aligned}
& \left|f_{\beta}\left(a^{x} ; g\right)\right| \Xi\left(a^{x}\right)^{-1}\left(1+\sigma\left(a^{x}\right)\right)^{m} \\
& \quad \leqslant c^{m+1}\left|f_{B}\left(a^{x} ; g\right)\right| \Xi(a)^{-1}(1+\sigma(a))^{m} \\
& \quad \leqslant c^{m+1}\left|\xi_{0}(a)\right|^{-1} \Xi(a)^{-1}(1+\sigma(a))^{m} \sum_{1 \leqslant i \leqslant p} \sum_{s \in W_{A}}\left|\eta_{i}(a) \beta^{s}\left(a ; u_{i}\right)\right|
\end{aligned}
$$

for $\beta \in C_{c}^{\infty}\left(A^{\prime}\right), a \in A^{++}, x \in C$ and $m \geqslant 0$. Put $A^{0}=\left(A^{++}\right)^{W_{A}}$. If $\beta \in C_{c}{ }^{\infty}\left(A^{0}\right)$, it is clear that $\operatorname{Supp} f_{\beta} \subset\left(A^{++}\right)^{C}$. Hence

$$
\begin{aligned}
& \sup _{x \in G}\left|f_{\beta}(x ; g)\right| \Xi(x)^{-1}(1+\sigma(x))^{m} \\
& \quad \leqslant c^{m+1} \sup _{a \in A^{++}}\left|\xi_{Q}(a) \Xi(a)\right|^{-1}(1+\sigma(a))^{m} \sum_{1 \leqslant i \leqslant p} \sum_{s \in W_{A}}\left|\eta_{i}(a) \beta^{s}\left(a ; u_{i}\right)\right|,
\end{aligned}
$$

for $\beta \in C_{c}^{\infty}\left(A^{0}\right)$ and $m \geqslant 0$. 


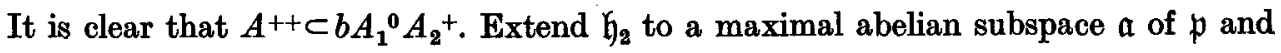
define an order on the dual of $\mathfrak{a}$, which is compatible with the one chosen on $\mathfrak{F}_{2}$. It is clear that

$$
\left|\xi_{0}(a)\right| \Xi(a)=\Xi\left(a_{2}\right) e^{e\left(\log a_{8}\right)} \quad(a \in A) .
$$

Since $a_{2} \in A_{2}{ }^{+}$for $a \in A^{++}$, it follows easily from [4 (j), Lemma 36] that

$$
\left|\xi_{\ell}(a)\right| \Xi(a) \geqslant 1
$$

for $a \in A^{++}$. Therefore since $R$ is stable under $W_{A}$, we obtain the following result from Lemma 30.

LEMMA 32. We can choose $m \geqslant 0, \eta_{i} \in R$ and $u_{i} \in \mathfrak{S}\left(\mathfrak{h}_{c}\right)(1 \leqslant i \leqslant p)$ such that

$$
\left|\int_{A} \beta \Phi d a\right| \leqslant \sum_{1 \leqslant i \leqslant p} \sup _{a \in A^{\prime}}(1+\sigma(a))^{m}\left|\eta_{i}(a) \beta\left(a ; u_{i}\right)\right|
$$

for $\beta \in C_{c}^{\infty}\left(A^{0}\right)$.

Moreover, we have the following lemma.

LEMмA 33. Let $\alpha \in Q$. Then

$$
\left|\eta_{\alpha}^{\top}(a) \alpha\left(\log a_{2}\right)\right| \leqslant 1+\left|\alpha\left(\log a_{2}\right)\right| \quad\left(a \in A^{\prime}\right) .
$$

Since $\left|c_{1}-c_{2}\right| \geqslant|| c_{1}|-| c_{2}||$, for two complex numbers $c_{1}, c_{2}$, it is clear that

$$
\left|\eta_{\alpha}(a) \alpha\left(\log a_{2}\right)\right| \leqslant\left|\alpha\left(\log a_{2}\right)\right|\left|1-e^{-\alpha\left(\log a_{2}\right)}\right|^{-1} \text {. }
$$

On the other hand,

$$
t\left(1-e^{-t}\right)^{-1}=t+t\left(e^{t}-1\right)^{-1}
$$

and

$$
t\left(e^{t}-1\right)^{-1} \leqslant 1 \quad(t \geqslant 0)
$$

Hence

$$
|t|\left|1-e^{-t}\right|^{-1} \leqslant 1+|t| \quad(t \in \mathbf{R})
$$

and this implies our assertion.

Fix a non-empty, open and connected subset $U$ of $b A_{1}{ }^{0}$ such that $U A_{2}$ meets $A^{++}$ and $\xi_{\alpha}(\alpha \in P)$ never takes the value 1 on $U$ unless $\alpha \in P_{z}$. Clearly this is possible. Then $U A_{2}{ }^{+} \subset A^{++} \subset b A_{1}{ }^{0} A_{2}{ }^{+} \subset A^{\prime}(R)$, where $A^{\prime}(R)$ is defined as in [4 (o), $\left.\$ 19\right]$. Also we know that $\Phi$ extends to an analytic function on $A^{\prime}(R)$ (see [4 (o), Lemma 31] and [4 (p), Lemma 64]). For $\delta \in C_{c}^{\infty}(U)$ and $\gamma \in C_{c}^{\infty}\left(\mathfrak{h}_{2}{ }^{+}\right)$, define the function $\delta \times \gamma$ in $C_{c}^{\infty}\left(A^{++}\right)$by

$$
(\delta \times \gamma)(a)=\delta\left(a_{1}\right) \gamma\left(\log a_{2}\right) \quad(a \in A) .
$$

Let $d a_{1}$ denote the Haar measure on $A_{1}$ and $d H$ the Euclidean measure on $\mathfrak{h}_{2}$. We normalize them in such a way that 
for $a=a_{1} \exp H \in A$. Put

$$
d a=d a_{1} d H
$$

$$
\Phi_{\delta}(H)=\int_{A_{1}} \Phi\left(a_{1} \exp H\right) \delta\left(a_{1}\right) d a_{1} \quad\left(H \in \mathfrak{h}_{2}{ }^{+}\right) .
$$

Then it is clear that $\quad \int_{A}(\delta \times \gamma) \cdot \Phi d a=\int_{\mathfrak{h}_{a}+} \Phi_{\delta} \gamma d H$

Now fix $\delta \in C_{c}^{\infty}(U)$ and put $V=\operatorname{Supp} \delta$. Then $V$ is a compact subset of $U$. Let $Q_{I}$ be the set of all imaginary roots (see $[4(n), \S 4]$ ) of $(\mathfrak{g}, \mathfrak{h})$. Then if $\alpha \in Q_{I}$, it follows from the definition of $U$, that $\eta_{\alpha}$ remains bounded on $V$. Let $P^{\prime}$ denote the complement of $P_{I}=P \cap Q_{I}$ in $P$. Put

$$
q(H)=\prod_{\alpha \in \mathcal{P}^{\prime}} \alpha(H) \quad\left(H \in \mathfrak{h}_{2}\right)
$$

Then $q$ is a polynomial function on $\mathfrak{h}_{2}$, which is not identically zero, and we conclude easily from Lemmas 32 and 33 that there exists an integer $m \geqslant 0$ such that the distribution

on $\mathfrak{h}_{2}+$ is tempered.

$$
T_{\delta}: \gamma \rightarrow \int_{\mathfrak{H}_{\mathrm{z}}+} \Phi_{\delta} q^{m} \gamma d H \quad\left(\gamma \in C_{c}^{\infty}\left(\mathfrak{h}_{2}{ }^{+}\right)\right)
$$

On the other hand, since $b A_{1}{ }^{\circ} A_{2}+\subset A^{\prime}(R)$ and $\Phi$ is analytic on $A^{\prime}(R)$, we conclude from [4 (e), Theorem 2], [4 (p), Lemma 64], and the 8-finiteness of $\Theta$, that

$$
\Phi\left(b a_{1} \exp H\right)=\sum_{1 \leqslant i \leqslant N} \xi_{i}\left(a_{1}\right) \sum_{1 \leqslant j \leqslant n} p_{i j}(H) e^{\lambda_{j}(H)} \quad\left(a_{1} \in{\left.A_{1}{ }^{0}, H \in \mathfrak{h}_{2}{ }^{+}\right)}\right.
$$

where $\xi_{i}(1 \leqslant i \leqslant N)$ are distinct characters of $A_{1}^{0}, \lambda_{j}(1 \leqslant j \leqslant n)$ distinct linear functions and $p_{i j}$ polynomial functions on $\mathfrak{h}_{2 c}$.

LEM MA 34. Fix $j(1 \leqslant j \leqslant n)$. Then $p_{i j}=0(1 \leqslant i \leqslant N)$ unless $\left(^{1}\right) \Re \lambda_{j}(H) \leqslant 0$ for all $H \in \mathfrak{h}_{2}{ }^{+}$.

For otherwise suppose that $\mathfrak{R} \lambda_{j}(H)>0$ for some $H \in \mathfrak{h}_{2}{ }^{+}$. Then it follows from [4 (p), Lemma 15] that

$$
\sum_{1 \leqslant i \leqslant N} p_{i j} \int_{A_{1}} \xi_{i}\left(a_{1}\right) \delta\left(b^{-1} a_{1}\right) d a_{1}=0 .
$$

Since this holds for every $\delta \in C_{c}{ }^{\infty}(U)$, we conclude from [4 (h), Lemma 20] that $p_{i j}=$ $0(1 \leqslant i \leqslant N)$.

It is now obvious from Lemma 34 that we can choose numbers $c, m \geqslant 0$ such that

$$
|\Phi(a)| \leqslant c(1+\sigma(a))^{m} \quad\left(a \in A^{++}\right)
$$

and this proves Theorem 7.

(1) $\Re c$ denotes the real part of a complex number $c$. 
We have still to prove Lemma 31. Put $\mathfrak{q}=[\mathfrak{g}, \mathfrak{h}]$ and for any $x \in G$, define the linear mapping $\Gamma_{x}$ of $\left(\mathfrak{S} \otimes(S)\right.$ into $\left(\mathfrak{S}\right.$ as in $[4(0), \S 2]$. Then if $a \in A^{\prime}, \Gamma_{a}$ defines a bijection of $\mathfrak{S}\left(\mathfrak{q}_{c}\right) \otimes \mathfrak{S}\left(\mathfrak{h}_{c}\right)$ onto $\mathbb{G}$ (see $\left[4(0)\right.$, Lemma 10]). Let $\gamma_{a}$ denote the inverse of this mapping.

LEMMA 35. Fix $g \in\left(\mathfrak{S}\right.$. Then we can choose $q_{i} \in S\left(\mathfrak{q}_{c}\right), u_{i} \in \subseteq\left(\mathfrak{h}_{\mathrm{c}}\right)$ and $\eta_{i} \in \mathbb{R}(1 \leqslant i \leqslant p)$ such that

$$
\gamma_{a}(g)=\sum_{1 \leqslant i \leqslant p} \eta_{t}(a)\left(q_{i} \otimes u_{i}\right) \quad\left(a \in A^{\prime}\right)
$$

We use the notation of $[4(0), \S 2]$ and put $d_{X}=L_{X}-R_{X}\left(X \in \mathfrak{g}_{c}\right)$. Then $d_{X}$ is a derivation of (S). Define $X_{\alpha}(\alpha \in Q)$ as in [4(n), § 4]. Then

$$
\sigma_{a}\left(X_{\alpha}\right)=\xi_{\alpha}(a)^{-1} L_{X_{\alpha}}-R_{X_{\alpha}}=-\left(1-\xi_{\alpha}(a)^{-1}\right) L_{X_{\alpha}}+d_{X_{\alpha}} \quad(a \in A)
$$

and therefore

$$
\eta_{\alpha}(a) \sigma_{a}\left(X_{\alpha}\right)=-\left(L_{X_{\alpha}}-\eta_{\alpha}(a) d_{X_{\alpha}}\right) \quad\left(a \in A^{\prime}\right)
$$

This shows that

$$
(-1)^{r} \eta_{\alpha_{1}}(a) \ldots \eta_{\alpha_{r}}(a) \Gamma_{a}\left(X_{\alpha_{1}} X_{\alpha_{2}} \ldots X_{\alpha_{r}} \otimes u\right)=\left(L_{X_{\alpha_{1}}}-\eta_{\alpha_{1}}(a) d_{X_{\alpha_{1}}}\right) \ldots\left(L_{X_{\alpha_{r}}}-\eta_{\alpha_{r}}(a) d_{X_{\alpha_{r}}}\right) u
$$

for $a \in A^{\prime}, u \in \mathfrak{S}\left(\mathfrak{h}_{c}\right)$ and $\alpha_{1}, \ldots, \alpha_{r} \in Q$. The assertion of the lemma now follows by an easy induction on the degree of $g$.

For any $f \in C^{\infty}(G)$, put $f(x: a)=f\left(a^{x}\right)(x \in G, a \in A)$.

Corollary. Let $f \in C^{\infty}(G)$. Then

$$
f\left(a^{x} ; g^{x}\right)=\sum_{1 \leqslant i \leqslant p} \eta_{i}(a) f\left(x ; q_{i}: a ; u_{i}\right) \quad\left(x \in G, a \in A^{\prime}\right)
$$

in the above notation.

This is obvious from [4 (o), Lemma 4].

We are now ready to prove Lemma 31. Let $g_{1}, \ldots, g_{N}$ be a base for the vector space over $\mathrm{C}$ spanned by $g^{x}(x \in G)$. Then

$$
g^{x^{-1}}=\sum_{1 \leqslant i \leqslant N} c_{i}^{\prime}(x) g_{i} \quad(x \in G)
$$

where $c_{i}^{\prime}$ are analyti functions on $G$. Hence

$$
f\left(a^{x} ; g\right)=\sum_{1 \leqslant i \leqslant N} c_{i}{ }^{\prime}(x) f\left(a^{x} ; g_{i}^{x}\right) \quad(x \in G, a \in A)
$$

for $f \in C^{\infty}(G)$. Therefore it follows from the corollary of Lemma 35, that we can choose analytic functions $c_{i}$ on $G$ and $q_{i} \in S_{\left(q_{c}\right)}, u_{i} \in \subseteq\left(\mathfrak{h}_{c}\right), \eta_{i} \in R(1 \leqslant i \leqslant p)$ such that 


$$
f\left(a^{x} ; g\right)=\sum_{1 \leqslant i \leqslant p} c_{i}(x) \eta_{i}(a) f\left(x ; q_{i}: a ; u_{i}\right) \quad\left(x \in G, a \in A^{\prime}\right)
$$

for any $f \in C^{\infty}(G)$. Put $\alpha(x)=\alpha^{*}\left(x^{*}\right)$ and $\alpha_{i}(x)=c_{i}(x) \alpha\left(x ; q_{i}\right) \quad(x \in G)$. Then it is clear that

$$
f_{\beta}\left(a^{x} ; g\right)=\sum_{1 \leqslant i \leqslant p} \alpha_{i}(x) \eta_{i}(a) \sum_{s \in W_{A}} \varepsilon(s) \beta^{s}\left(a ; u_{i} \circ \Delta^{-1}\right) \quad\left(x \in C, a \in A^{\prime}\right)
$$

for $\beta \in C_{c}^{\infty}\left(A^{\prime}\right)$. Let $u \rightarrow^{\prime} u$ denote the automorphism of $\subseteq\left(\mathfrak{h}_{\mathrm{c}}\right)$ such that ${ }^{\prime} H=H-\varrho(H)$ $\left(H \in \mathfrak{h}_{c}\right)$. Then since $\Delta^{-1}=\xi_{g}^{-1} \eta$, it is clear that

$$
u \circ \Delta^{-1}=\xi_{0}^{-1 '} u \circ \eta \quad\left(u \in \subseteq\left(\mathfrak{h}_{c}\right)\right),
$$

as a differential operator on $A^{\prime}$. Therefore since $R$ is stable under both $\subseteq\left(\mathfrak{h}_{c}\right)$ and $W_{A}$, Lemma 31 follows immediately from the compactness of $C$.

\section{\$ 20. Proof of Theorem 8}

Now suppose $\operatorname{rank} G=\operatorname{rank} K$ and fix a Cartan subgroup $B$ of $G$ such that $B \subset K$. Define $L$ and $\Theta_{\lambda}(\lambda \in L)$ as in [4 (q), § 8]. Then it follows from Theorem 7 and [4 (p), Lemma 52] that $\Theta_{\lambda}$ is tempered. Similarly we conclude from [4 (p), § 29] that

$$
f \rightarrow \sum_{\lambda \in L}\left|\varpi(\lambda) \Theta_{\lambda}(f)\right| \quad(f \in \mathbb{C}(G))
$$

is a continuous mapping of $\mathcal{C}(G)$ into $\mathbf{C}$. Therefore

is a tempered distribution.

$$
f \rightarrow \sum_{\lambda \in L} \varpi(\lambda) \Theta_{\lambda}(f) \quad(f \in \mathcal{C}(G))
$$

Now we use the notation of [4 (q), § 14]. It follows from [4 (q), Lemma 27 and Cor. 1 of Lemma 57] that $T_{A}$ is a tempered distribution on $\mathrm{C}\left(\tilde{A}_{R}\right)$. Define

$$
\phi_{f}(h)=F_{f}\left(h ; \varpi_{0}\right) \quad\left(h \in \tilde{A}_{R}\right)
$$

for $f \in \mathrm{C}(G)$. Then it follows from $\S 18$ that $f \rightarrow \phi_{f}$ is a continuous mapping of $\mathcal{C}(G)$ into $\mathrm{C}\left(\tilde{A}_{R}\right)$ and therefore

$$
f \rightarrow T_{A}\left(\phi_{f}\right) \quad(f \in \mathcal{C}(G))
$$

is a tempered distribution on $G$. Hence we obtain the following result from [4 (q), Theorem 3].

ThEOREM 8. Let $f \in \mathcal{C}(G)$. Then

$$
c f(1)=(-1)^{q} \sum_{\lambda \in L} \varpi(\lambda) \Theta_{\lambda}(f)+\sum_{2 \leqslant i \leqslant r} T_{i}\left(\phi_{f, i}\right)
$$

in the notation of [4 (q), Theorem 3]. 
Let $J(G)$ denote the subspace of all $f \in C(G)$ which are invariant under left and right translations of $K$. It follows from Lemma 19 that $I_{c}{ }^{\infty}(G)=\mathcal{J}(G) \cap C_{c}{ }^{\infty}(G)$ is dense in $\mathcal{I}(G)$. Moreover if we take into account Lemma 18 and [4 (j), Theorem 3 and Lemma 36], it follows without difficulty that the space $J(G)$, including its topology, is the same as $I(G)$ of $[4(k), \S 12]$.

Corollary. Let $f \in \mathcal{J}(G)$. Then, if $G$ is not compact,

$$
c f(1)=\sum_{2 \leqslant i \leqslant r} T_{\mathfrak{i}}\left(\phi_{f, i}\right)
$$

This is an immediate consequence of $[4(q)$, Theorem 6$]$.

\section{§ 21. Proof of an earlier conjecture}

We now drop the assumption that $\operatorname{rank} G=\operatorname{rank} K$. Let $\mathfrak{a}$ be a maximal abelian subspace of $\mathfrak{p}$. Define $A, N, \mathfrak{\pi}$ and $\sigma$ as in $[4(q), \S 16]$ and fix a Haar measure $d n$ on $N$. Put

$$
\Phi_{f}(a)=e^{\sigma n \log a)} \int_{N} f(a n) d n \quad(a \in A)
$$

for $f \in \mathcal{J}(G)$. Then we know from Theorem 3 that $f \rightarrow \Phi_{f}$ is a continuous mapping of $\mathcal{J}(G)$ into $\mathrm{C}(A)$.

LEMMA 36. Let $f$ be an element in $\mathcal{I}(G)$ such that $\Phi_{f}=0$. Then $f=0$.

We shall prove this by induction on $\operatorname{dim} G$. However we first verify the following result.

Lемма 37. It is sufficient to show that, under the conditions of Lemma $36, f(1)=0$.

Fix an element $\alpha \in I_{c}^{\infty}(G)$ and consider $f_{0}=\alpha * f$. Then

$$
f_{0}=\int_{G} \alpha(x) l(x) f d x \in \mathcal{J}(G)
$$

from the results of $\S 10$. Let $d a$ denote the Haar measure on $A$. Then we can assume that

and therefore

$$
d x=e^{2 \sigma(\log a)} d k d a d n \quad(x=k a n, k \in K, a \in K, n \in N)
$$

$$
f_{0}(y)=\int_{G} \alpha\left(x^{-1}\right) f(x y) d x=\int e^{2 \sigma(\log a)} \alpha\left(n^{-1} a^{-1}\right) f(a n y) d a d n \quad(y \in G) .
$$

Hence it follows without difficulty that 


$$
\Phi_{f_{0}}\left(a_{0}\right)=\int_{A} \Phi_{\alpha}(a) \Phi_{f}\left(a^{-1} a_{0}\right) d a=0 \quad\left(a_{0} \in A\right)
$$

and so we conclude that $\quad \int \alpha\left(x^{-1}\right) f(x) d x=f_{0}(1)=0$.

Since this holds for every $\alpha \in I_{c}^{\infty}(G)$, it is obvious that $f=0$.

We shall now undertake some preparation for the proof of Lemma 36. Let $B=\theta(B)$ be a Cartan subgroup of $G$ and $\mathfrak{b}$ its Lie algebra. For any $f \in \mathcal{C}(G)$, define $F_{f}$ corresponding to $B$ as in $\S 18$. Let $\mathfrak{m}$ be the centralizer of $\mathfrak{b} \cap \mathfrak{p}$ in $\mathfrak{g}$ and $M$ the analytic subgroup of $G$ corresponding to $\mathrm{m}$. Define the differential operator $\varpi$ on $B$ as in $[4,(0), \S 22]$.

LEMMA 38. The statements of [4 (o), Lemma 52] remain true for $f \in \mathrm{C}(G)$. Moreover, if $\mathfrak{b}$ is fundamental in $\mathfrak{g}$, there exists a positive number $c$ such that

$$
c f(1)=(-1)^{q} F_{f}(1 ; \varpi)
$$

for $f \in \mathrm{C}(G)$. Here $q=\frac{1}{2}(\operatorname{dim} G / K-\operatorname{rank} G+\operatorname{rank} K)$.

The first part follows from Theorem 3, its corollary and Lemma 29. The second is a consequence of [4 (o), §22], [4 (i), p. 759] and [4 (n), Lemmas 17 and 18].

Since $\mathfrak{a}$ is maximal abelian in $\mathfrak{p}$, we can choose $k \in K$ such that $(\mathfrak{b} \cap \mathfrak{p})^{k} \subset \mathfrak{a}$. Hence we may assume that $\mathfrak{b} \cap \mathfrak{p} \subset \mathfrak{a}$. Put $\mathfrak{n}_{1}=\mathfrak{m} \cap \mathfrak{n}, N_{1}=M \cap N, K_{1}=M \cap K$ and

$$
\sigma_{1}(H)=\operatorname{tr}(\operatorname{ad} H)_{\mathfrak{n}_{1}} \quad(H \in \mathfrak{a}) .
$$

For any $f \in \mathcal{J}(G)$, define $u_{f}$ as in Lemma 22 (for $\mathfrak{h}=\mathfrak{b}$ ). Then it is clear that $u_{f}$ is bi-invariant under $K_{1}$ and therefore $u_{f} \in \mathcal{J}(M)$. The following lemma is a simple consequence of the definition of $u_{f}$.

LEм м 39. The Haar measure $d n_{1}$ on $N_{1}$ can be so normalized that

for $f \in \mathcal{I}(G)$

$$
\Phi_{f}(a)=e^{\sigma_{1}(\log a)} \int_{N_{1}} u_{f}\left(a n_{1}\right) d n_{1} \quad(a \in A)
$$

Now we come to the proof of Lemma 36. We may obviously assume that $G$ is not compact. Fix $f \in \mathfrak{J}(G)$ such that $\Phi_{f}=0$ and first suppose that $\mathfrak{m} \neq \mathrm{g}$. Then the induction hypothesis is applicable to $M$ and therefore we conclude from Lemma 39 that $u_{f}=0$. But then $F_{f}=0$ from Lemma 29.

In view of Lemma 37, it would be sufficient to verify that $f(1)=0$. We now use the notation of the proof of Lemma 26. Let $f_{1}$ be the restriction of $f$ on $G_{1}$. If $\mathfrak{c}_{\mathfrak{p}} \neq\{0\}$, our induction hypothesis is applicable to $G_{1}$ and therefore $f(1)=f_{1}(1)=0$. So we may assume 
that $\mathfrak{c}_{\mathfrak{p}}=\{0\}$. Choose $\mathfrak{b}$ so that it is fundamental in $\mathfrak{g}$. If rank $\mathfrak{g}>$ rank $\mathfrak{f}$, it is clear that $\mathfrak{b} \cap \mathfrak{p} \neq\{0\}$. Therefore since $\mathfrak{c}_{\mathfrak{p}}=\{0\}$, it follows that $\mathfrak{m} \neq \mathfrak{g}$. But as we have seen above, this implies that $F_{f}=0$ and therefore again $f(1)=0$ from Lemma 38 .

So we may now suppose that rank $\mathfrak{f}=$ rank $\mathfrak{g}$. Then $\mathfrak{b} \cap \mathfrak{p} \notin \mathfrak{c}$, if $\mathfrak{b}$ is not fundamental in $\mathfrak{g}$, and therefore $F_{f}=0$. Hence it follows immediately from the corollary of Theorem 8 that $f(1)=0$. This completes the proof of Lemma 36 .

In $[4(\mathrm{k}), \S 16]$ the proof of the Plancherel formula for $G / K$ was reduced to two conjectures. The first of these has been verified by Gindikin and Karpelevič [3] (see also [5 (b), § 3]) while Lemma 36 proves the second. Hence in particular, [4 (k), Corollary 2, p. 611] holds for all $f \in \mathcal{J}(G)$.

\section{§ 22. Proof of Lemma 40 (first part)}

We return to the notation of $\S 18$ except that we write $B=A_{\mathfrak{h}}$. Let $d h$ denote the Haar measure on $B$. Fix a finite subset $F$ of $\mathcal{E}_{K}$ and define $\alpha_{F}$ as in $\S 12$. Put ${ }_{F} \mathrm{C}(G)=\alpha_{F} * \mathrm{C}(G)$. It is obvious that ${ }_{F} \mathrm{C}(G)$ consists exactly of those $f \in \mathrm{C}(G)$ for which $\alpha_{F} * f=f$.

L Емм A 40. Given $r_{0} \geqslant 0$ and $u \in \subseteq\left(\mathfrak{h}_{c}\right)$, we can choose $r \geqslant 0$ and a finite set of elements $g_{1}, \ldots, g_{p}$ in (S) such that

for all $f \in_{F} \mathrm{C}(G)$.

$$
\int_{B}(1+\sigma(h))^{r_{0}}\left|F_{f}(h ; u)\right| d h \leqslant \sum_{1 \leqslant i \leqslant p} \int_{G}\left|g_{i} f\right| \Xi(1+\sigma)^{r} d x
$$

First suppose $\mathfrak{h} \cap \mathfrak{l}=\{0\}$. Then $B \subset K$ and

$$
\int_{B}\left|F_{f}(h ; u)\right| d h \leqslant \sup _{h \in B^{\prime}}\left|F_{f}(h ; u)\right|
$$

provided the total measure of $B$ is 1 . Let $\phi_{B}$ denote the characteristic function of $G_{B}=\left(B^{\prime}\right)^{G}$. Then it follows from [4 (o), Lemma 41 and Theorem 3 ] and Lemma 25 that there exist elements $z_{1}, \ldots, z_{p} \in 3$ such that

$$
\sup _{h \in B^{\prime}}\left|F_{f}(h ; u)\right| \leqslant \sum_{1 \leqslant i \leqslant p} \int_{G}\left|z_{i} f\right| \phi_{B} d x \quad(f \in \mathcal{C}(G)) .
$$

Now suppose $g \in_{F} \mathrm{C}(G)$. Then $g=\alpha_{F} * g$ and therefore

$$
|g(x)| \leqslant\left|\alpha_{F}\right|_{\infty} g_{1}(x) \quad(x \in G),
$$

where $\left|\alpha_{F}\right|_{\infty}=\sup \left|\alpha_{F}\right|$ and $\quad g_{1}(x)=\int_{K}|g(k x)| d k$. 
Therefore $\quad \int|g| \phi_{B} d x \leqslant\left|\alpha_{F}\right|_{\infty} \int g_{1} \phi_{B} d x \leqslant c \int|g| \Xi d x \quad\left(g \in_{F} \mathrm{C}(G)\right)$

from [4 (q), Theorem 5], where $c$ is a positive number independent of $g$. Hence

$$
\sup _{h \in B^{\prime}}\left|F_{f}(h ; u)\right| \leqslant c \sum_{1 \leqslant i \leqslant p} \int\left|z_{i} f\right| \Xi d x \quad\left(f \in \in_{F} \mathrm{C}(G)\right)
$$

and this implies our assertion in this case.

Now, in order to prove the lemma in general, we use induction on $\operatorname{dim} G$. Let us keep to the notation of the proof of Lemma 26 and first assume that $c_{\mathfrak{p}} \neq\{0\}$. We can obviously suppose that $u=\gamma u_{1}$ where $\gamma \in \mathfrak{C}_{\mathfrak{p}}$ and $u_{1} \in \mathfrak{S}\left(\mathfrak{h}_{1 c}\right)$. For $f \in \mathcal{C}(G)$ and $c \in C_{\mathfrak{p}}$, let $f_{c}$ denote the function on $G_{1}$ given by

$$
f_{c}(x)=(1+\sigma(c))^{r_{0}} f(c x ; \gamma) \quad\left(x \in G_{1}\right)
$$

Then $f_{c} \in \mathrm{C}\left(G_{1}\right)$. Moreover, since $K \subset G_{1}$, it is obvious that $f_{c} \epsilon_{F} \mathrm{C}\left(G_{1}\right)$ if $f \in_{F} \mathrm{C}(G)$. Finally

$$
(1+\sigma(c h))^{r_{0}}\left|F_{f}(c h ; u)\right| \leqslant(1+\sigma(h))^{r_{0}}\left|F_{f c}\left(h ; u_{1}\right)\right|
$$

for $c \in C_{\mathfrak{p}}$ and $h \in B_{1}^{\prime}=B^{\prime} \cap G_{1}$. Therefore our assertion follows immediately by applying the induction hypothesis to $G_{1}$ and observing that

$$
\max (\sigma(c), \sigma(x)) \leqslant \sigma(c x) \quad\left(c \in C_{\mathfrak{p}}, x \in G_{1}\right)
$$

So we may now suppose that $\mathfrak{c}_{\mathfrak{p}}=\{0\}$ and $\mathfrak{h} \cap \mathfrak{p} \neq\{0\}$. Then the induction hypothesis is applicable to $M$.

\section{\$ 23. Proof of Lemma 40 (second part)}

Let $d m$ denote the Haar measure on $M$. Define $\Xi_{M}, u_{f}(f \in \mathrm{C}(G))$ and $Z_{B}$ as in $\$ \S 14,15$ and Lemma 29 respectively. For $b \in Z_{B}$ and $f \in C(G)$, put

$$
f_{b}(x)=\int_{K} f\left(k b x k^{-1}\right) d k \quad(x \in G) .
$$

Since $Z_{B} \subset K$, it is obvious that if $f \epsilon_{F} C(G)$, the same holds for $f_{b}$.

Lемм А 41. Fix $r \geqslant 0$ and $\zeta \in \subseteq\left(\mathfrak{m}_{c}\right)$. Then we can choose $v_{1}, \ldots, v_{p}$ in $\mathfrak{G}$ such that

$$
\int_{M}\left|\zeta u_{f_{b}}\right| \Xi_{M}(1+\sigma)^{r} d m \leqslant \sum_{1 \leqslant i \leqslant p} \int_{G}\left|v_{i} f\right| \Xi(1+\sigma)^{r} d x
$$

for $f \in_{F} \mathrm{C}(G)$ and $b \in Z_{B}$.

4-662900. Acta mathematica. 116. Imprimé le 10 juin 1966. 
Put $N_{2}=N_{\mathfrak{\zeta}}$ in the notation of $\S 14$. We have seen in $\S 15$ that $\zeta u_{g}=u_{\zeta g}(g \in \mathcal{C}(G))$ where ' $\zeta=d \zeta \circ d^{-1}$. Fix $b \in Z_{B}$. Then if $f \epsilon_{F} \mathcal{C}(G)$, it is obvious that $f^{\prime}=' \zeta f_{b}$ is also in ${ }_{F} \mathrm{C}(G)$ and

$$
\left|f^{\prime}(x)\right| \leqslant\left|\alpha_{F}\right|_{\infty} \int_{E}\left|f^{\prime}(k x)\right| d k=\left|\alpha_{F}\right|_{\infty} \int_{E \times K}\left|f\left(k_{1} x k_{2}^{-1} ; \zeta^{\prime} \zeta^{k_{2}}\right)\right| d k_{1} d k_{2} .
$$

Let $\zeta_{1}, \ldots, \zeta_{p}$ be a base for the subspace of (S) spanned by ' $\zeta^{k}(k \in K)$. Then

$$
\zeta^{k}=\sum_{1 \leqslant i \leqslant p} a_{i}(k) \zeta_{i} \quad(k \in K),
$$

where $a_{i}$ are continuous functions on $K$. Put

$$
c_{1}=\max _{i} \sup \left|a_{i}\right|
$$

and $c_{2}=\left|\alpha_{F}\right|_{\infty} c_{1}$. Then it is clear that

$$
\left|f^{\prime}(x)\right| \leqslant c_{2} \sum_{i} \int_{K \times K}\left|f_{i}\left(k_{1} x k_{2}\right)\right| d k_{1} d k_{2},
$$

where $f_{i}=\zeta_{i} f$.

On the other hand, it is clear that

$$
\int\left|\zeta u_{f_{b}}\right| \Xi_{M}(1+\sigma)^{r} d m \leqslant \int_{M \times N_{1}}\left|f^{\prime}(m n)\right| d(m) \Xi_{M}(m)(1+\sigma(m))^{r} d m d n .
$$

By Lemma 21, there exists a number $c_{0} \geqslant 1$ such that

$$
1+\sigma(m) \leqslant c_{0}(1+\sigma(m n)) \quad\left(m \in M, n \in N_{2}\right) .
$$

Put $c_{3}=c_{0}{ }^{r} c_{2}$. Then

$$
\int\left|\zeta u_{f_{b}}\right| \Xi_{M}(\mathbf{1}+\sigma)^{r} d m \leqslant c_{3} \int f_{0}(x) e^{-\varrho\left(H_{2}(x)\right)} \Xi_{M}(\mu(x))(1+\sigma(x))^{r} d x
$$

in the notation of $\S 42$, where

$$
f_{0}(x)=\sum_{1 \leqslant i \leqslant p} \int_{K \times R}\left|f_{i}\left(k_{1} x k_{2}\right)\right| d k_{1} d k_{2}
$$

But we know from the corollary of Lemma 84 that

$$
\int_{\Sigma} e^{-\varrho\left(H_{2}(x k)\right)} \Xi_{M}(\mu(x k)) d k=\Xi(x) \quad(x \in G)
$$

Hence it is clear that 


$$
\int\left|\zeta u_{f_{b}}\right| \Xi_{M}(1+\sigma)^{r} d m \leqslant c_{3} \sum_{i} \int\left|\zeta_{i} f\right| \Xi(1+\sigma)^{r} d x
$$

and this implies the assertion of the lemma.

We can now finish the proof of Lemma 40. Let $F_{1}$ be the set of all irreducible classes of $K_{1}=K \cap M$, which occur in the reduction, with respect to $K_{1}$, of some element of $F$. It is clear from Lemma 22 that $u_{f} \epsilon_{F_{1}} \mathrm{C}(M)$ if $f \epsilon_{F} \mathrm{C}(G)$. Since the induction hypothesis is applicable to $M$, we can choose $\zeta_{1}, \ldots, \zeta_{q}$ in $\subseteq\left(\mathfrak{m}_{c}\right)$ and $r \geqslant 0$ such that

$$
\int_{B_{0}}\left|F_{g}{ }^{M}(h ; u)\right|(1+\sigma(h))^{r_{0}} d h \leqslant \sum_{1 \leqslant i \leqslant p} \int_{M}\left|\zeta_{i} g\right| \Xi_{M}(1+\sigma)^{r} d m
$$

for $g \epsilon_{F_{1}} C(M)$. (Here $B_{0}=B \cap M$ and $F_{g}{ }^{M}$ is defined as in Lemma 29.) Therefore the required result follows immediately from Lemmas 29 and 41 .

\section{\$ 24. Proof of Theorem 9}

Let $\Theta$ be an invariant and 8 -finite distribution on $G$. Fix $\delta \in \mathcal{E}_{K}$ and let $\Theta_{\mathfrak{d}}$ denote the corresponding Fourier component of $\Theta$ (see $[4(q), \S 17]$ ). Then we know from [4 (q), Lemma 33] that $\Theta_{b}$ is an analytic function on $G$.

THEOR м 9. Suppose $\Theta$ is tempered. Then we can choose $c, m \geqslant 0$ such that

$$
\left|\Theta_{\diamond}(x)\right| \leqslant c \Xi(x)(1+\sigma(x))^{m} \quad(x \in G) .
$$

It follows from Theorem 1 that we can choose $\beta^{\prime} \in C_{c}^{\infty}(G)$ such that $\Theta_{\triangleright}=\Theta_{\delta} * \beta^{\prime}$. Put $\beta(x)=\beta^{\prime}\left(x^{-1}\right)$. Then

$$
\Theta_{\triangleright}(f)=\Theta_{\triangleright}(f * \beta) \quad\left(f \in C_{c}^{\infty}(G)\right)
$$

On the other hand, from Theorem 7 and its corollary, we can choose numbers $c_{0}, s \geqslant 0$ such that

$$
|\Theta(g)| \leqslant c_{0} \sum_{1 \leqslant i \leqslant r} \int_{A_{i}}(\mathrm{I}+\sigma(a))^{s}\left|F_{g, i}(a)\right| d_{i} a \quad\left(g \in C_{c}^{\infty}(G)\right)
$$

Therefore from Iemma 40 , there exist elements $v_{1}, \ldots, v_{p} \in \mathbb{S}$ and $m \geqslant 0$ such that

$$
\left|\Theta_{\mathfrak{b}}(g)\right|=\left|\Theta\left(g^{\prime}\right)\right| \leqslant \sum_{1 \leqslant i \leqslant p} \int\left|v_{i} g^{\prime}\right| \Xi(1+\sigma)^{m} d x \leqslant d(\delta)^{2} \sum_{i} \int\left|v_{i} g\right| \Xi(1+\sigma)^{m} d x .
$$

Here $g^{\prime}=\alpha_{\S} * g$ and we have made use of the fact that

$$
\Theta_{\mathfrak{D}}(g)=\Theta\left(g * \alpha_{\mathfrak{D}}\right)=\Theta\left(\alpha_{\mathfrak{D}} * g\right)
$$


which follows from the invariance of $\Theta$. Therefore if we put $\beta_{i}=v_{i} \beta$, we get

$$
\left|\Theta_{\triangleright}(f)\right|=\left|\Theta_{\triangleright}(f * \beta)\right| \leqslant \sum_{i} d(\mathfrak{D})^{2} \int\left|f * \beta_{i}\right| \Xi(1+\sigma)^{m} d x \quad\left(f \in C_{c}^{\infty}(G)\right)
$$

Now put $\Omega=\operatorname{Supp} \beta$ and $\quad c_{1}=d(\delta)^{2} \sum_{i} \sup \left|\beta_{i}\right|$.

Then

$$
\left|\Theta_{b}(f)\right| \leqslant c_{1} \int_{\Omega}(1+\sigma(y))^{m} d y \int_{G}|f(x)| \Xi(x y)(1+\sigma(x))^{m} d x
$$

But we can choose (see $\left[4(q)\right.$, Lemma 32]) $c_{2} \geqslant 0$ such that

$$
\Xi(x y) \leqslant c_{2} \Xi(x) \quad(x \in G, y \in \Omega) \text {. }
$$

Then

$$
c=c_{1} c_{2} \int_{\Omega}(1+\sigma(y))^{m} d y<\infty
$$

and

$$
\left|\Theta_{b}(f)\right| \leqslant c \int_{G}|f| \Xi(1+\sigma)^{m} d x
$$

for $f \in C_{c}{ }^{\infty}(G)$. The assertion of Theorem 9 is now obvious.

Corollary. Let $f \in \mathcal{C}(G)$. Then

$$
\Theta_{\triangleright}(f)=\int_{G} \Theta_{\triangleright} f d x
$$

Let $\alpha$ be a variable element in $C_{c}^{\infty}(G)$ which converges to $f$ in $\mathcal{C}(G)$. Then

from Lemma 11.

$$
\Theta_{\mathfrak{b}}(f)=\lim \Theta_{\mathfrak{D}}(\alpha)=\lim \int \Theta_{\mathfrak{b}} \alpha d x=\int \Theta_{\mathfrak{D}} f d x
$$

\section{\$ 25. Application to tempered representations}

Let $\pi$ be a representation of $G$ on a locally convex space $\mathfrak{H}$. We say that an element $\phi \in \mathfrak{S}_{\text {is }} K$-finite (under $\pi$ ), if the space spanned by the vectors $\pi(k) \phi(k \in K)$ has finite dimension. Now suppose $\mathfrak{S}$ is a Hilbert space and $\pi$ is unitary and irreducible. Let $\Theta_{\pi}$ denote the character of $\pi$ (see [4 (b), $\S 5]$ and [8]). We say that $\pi$ is tempered if $\Theta_{\pi}$ is tempered.

THEOREM 10. Let $\pi$ be an irreducible unitary representation of $G$ on a Hilbert space $\mathfrak{S}$, which is tempered. Then there exists a number $m \geqslant 0$ with the following property. For any two $K$-finite vectors $\phi, \psi \in \mathfrak{g}$, we can choose a constant $c \geqslant 0$ such that 


$$
|\langle\phi, \pi(x) \psi)| \leqslant c \Xi(x)(1+\sigma(x))^{m} \quad(x \in G) .
$$

Let $\Theta$ denote the character of $\pi$. Then $\Theta$ is an invariant eigendistribution of 8 on $G$. Define $E_{\mathrm{b}}\left(\delta \in \mathcal{E}_{R}\right)$ as in $\S 2$ and put $\mathfrak{H}_{\mathrm{b}}=E_{\mathrm{b}} \mathfrak{S}$. Choose an orthonormal base $\psi_{i}(i \in J)$ for $\mathfrak{H}$ such that every $\psi_{i}$ lies in $\mathfrak{S}_{\mathfrak{b}}$ for some $\delta$. Let $J_{\mathfrak{b}}$ be the set of all $i$ such that $\psi_{i} \in \mathfrak{S}_{\mathfrak{b}}$. Then ()

$$
\left[J_{\mathfrak{b}}\right]=\operatorname{dim} \mathfrak{h}_{\mathfrak{b}} \leqslant N d(\mathfrak{D})^{2} \quad\left(d \in \mathcal{E}_{\mathbb{K}}\right),
$$

where $N$ is a positive integer independent of $\delta$ (see [4 (b), Theorem 4]). It follows from the definition of $\Theta$ that

$$
\Theta(f)=\sum_{i \in J} \int f(x)\left(\psi_{i}, \pi(x) \psi_{i}\right) d x \quad\left(f \in C_{c}^{\infty}(G)\right)
$$

and therefore it is clear that

$$
\Theta_{\mathrm{\jmath}}(x)=\operatorname{tr}\left(E_{\mathrm{\jmath}} \pi(x) E_{\mathrm{\wp}}\right) \quad(x \in G)
$$

for $\mathfrak{D} \in \mathcal{E}_{K}$. Now fix $\mathfrak{D}_{0} \in \mathcal{E}_{E}$ such that $\mathfrak{W}_{\mathfrak{D}_{0}} \neq\{0\}$. Then, by Theorem 9 , we can choose numbers $c_{0}, m \geqslant 0$ such that

$$
\left|\Theta_{b_{0}}(x)\right| \leqslant c_{0} \Xi(x)(1+\sigma(x))^{m} \quad(x \in G) .
$$

Let $\phi$ and $\psi$ be two $K$-finite elements in $\mathfrak{S}$. For any finite subset $F$ of $\mathcal{E}_{K}$, put

$$
E_{F}=\sum_{\mathfrak{b} \in F} E_{\mathrm{b}}
$$

and $\mathfrak{S}_{F}=E_{F} \mathfrak{H}_{\mathrm{S}}$. Also define $\alpha_{F}$ as in $\S 6$. We can obviously choose $F$ so large that $\mathfrak{D}_{0} \in F$ and $\phi, \psi$ lie in $\tilde{S}_{F}$. Let $\mathcal{L}_{F}(G)$ be the space of all functions $f \in C_{c}{ }^{\infty}(G)$ such that $f=\alpha_{F} * f * \alpha_{F}$. Then $\mathcal{L}_{F}(G)$ is an algebra under convolution and it is clear that $\mathfrak{S}_{F}$ is stable under $\pi(f)$ for $f \in \mathcal{L}_{F}(G)$. Let $\pi_{F}(f)$ denote the restriction of $\pi(f)$ on $\mathfrak{S}_{F}$. Then $\pi_{F}$ is a representation of $\mathcal{L}_{F}(G)$ on $\mathfrak{h}_{F}$. We claim that this representation is irreducible. Fix an element $\psi_{0} \neq 0$ in $\mathfrak{S}_{F}$. Since $\mathfrak{S}$ is irreducible under $\pi$, elements of the form $\pi(f) \psi_{0}\left(f \in C_{c}{ }^{\infty}(G)\right)$ are dense in $\mathfrak{S}$. Therefore, since

$$
E_{F} \pi(f) \psi_{0}=\pi\left(\alpha_{F} * f * \alpha_{F}\right) \psi_{0}
$$

and $\operatorname{dim} \mathfrak{S}_{F}<\infty$, it is clear that $\mathfrak{S}_{F}=\pi\left(\mathcal{L}_{F}(G)\right) \psi_{0}$ and this shows that $\pi_{F}$ is irreducible. Hence, by the Burnside theorem, we can choose $\alpha, \beta \in \mathcal{L}_{F}(G)$ such that

$$
\phi=\pi(\alpha) \psi_{i}, \psi=\pi(\beta) \psi_{i} \quad\left(i \in J_{D_{0}}\right) .
$$

Therefore $\quad(\phi, \pi(x) \psi)=\left(\psi_{i}, \pi(\hat{\alpha}) \pi(x) \pi(\beta) \psi_{i}\right) \quad\left(x \in G, i \in J_{\mathfrak{b}_{0}}\right)$

where $\alpha(y)=$ conj $\alpha\left(y^{-1}\right)(y \in G)$. This shows that

(1) As usual, $[F]$ denotes the number of elements in a set $F$. 


$$
(\phi, \pi(x) \psi) . \operatorname{dim} \mathfrak{S}_{\mathfrak{D}_{0}}=\operatorname{tr}\left(E_{\delta_{0}} \pi(\dot{\alpha}) \pi(x) \pi(\beta) E_{\delta_{0}}\right)=\int_{G \times G} \tilde{\alpha}(y) \Theta_{\mathfrak{D}_{0}}(y x z) \beta(z) d y d z
$$

and the required result now follows immediately from Lemma 10 and [4 (q), Lemma 32], if we observe that $\mathfrak{S}_{\mathfrak{o}_{0}} \neq\{0\}$.

\section{Part II. Spherical functions and differential equations}

\section{\$ 26. Two key lemmas and their first reduction}

Let $V$ be a (complex) Hilbert space of finite dimension and $\mu=\left(\mu_{1}, \mu_{2}\right)$ a continuous and unitary double representation $\left({ }^{1}\right)$ of $K$ on $V$. Define $c_{\mathfrak{p}}$ as in $\S 7$.

LEMMA 42. Let $\phi \neq 0$ be a $C^{\infty} \mu$-spherical function (see $\left.\S 11\right)$ from $G$ to $V$ such that:

1) The space of functions of the form $z \phi(z \in \Re)$ has finite dimension.

2) There exist numbers $c, r \geqslant 0$ such that

$$
|\phi(x)| \leqslant c \Xi(x)(1+\sigma(x))^{r} \quad(x \in G) .
$$

Put

$$
\|\phi\|_{t}=\left\{\int_{\sigma(x) \leqslant t}|\phi(x)|^{2} d x\right\}^{\frac{1}{2}} \quad(t \geqslant 0) .
$$

Then there exists a unique integer $\nu \geqslant 0$ such that

$$
0<\liminf _{t \rightarrow \infty} t^{-v / 2}\|\phi\|_{t} \leqslant \limsup _{t \rightarrow \infty} t^{-v / 2}\|\phi\|_{t}<\infty .
$$

Moreover, $\boldsymbol{v} \geqslant \operatorname{dim} \mathfrak{c}_{\mathfrak{p}}$ and, for any $\varepsilon>0$, we can choose $t_{0}, \delta>0$ such that

for $t_{0} \leqslant t_{1} \leqslant t_{2} \leqslant(1+\delta) t_{1}$.

$$
t_{1}^{-v}\left\{\|\phi\|_{t_{2}}^{2}-\|\phi\|_{t_{1}}^{2}\right\} \leqslant \varepsilon
$$

Fix a maximal abelian subspace $\mathfrak{a}_{\mathfrak{p}}$ of $\mathfrak{p}$ and let $A_{\mathfrak{p}}=\exp \mathfrak{a}_{\mathfrak{p}}$ be the corresponding subgroup of $G$. Introduce an order in the space of (real) linear functions on $\mathfrak{a}_{\mathfrak{p}}$ and let $\mathfrak{g}=\mathfrak{l}+\mathfrak{a}_{\mathfrak{p}}+\mathfrak{n}$ be the corresponding Iwasawa decomposition of $\mathfrak{g}$. As usual put

$$
\varrho(H)=\frac{1}{2} \operatorname{tr}(\operatorname{ad} H)_{\mathfrak{n}} \quad\left(H \in \mathfrak{a}_{\mathfrak{p}}\right)
$$

and let $\mathfrak{a}_{\mathfrak{p}}+$ be the set of all $H \in \mathfrak{a}_{\mathfrak{p}}$ where $\alpha(H) \geqslant 0$ for every positive root $\alpha$ of $\left(\mathfrak{g}, \mathfrak{a}_{\mathfrak{p}}\right)$. We recall (see $\S 7$ ) that $\mathfrak{p}$ is a Hilbert space with respect to the norm $\|X\|(X \in \mathfrak{p})$. Consider the set $S^{+}$of all points $H \in \mathfrak{a}_{\mathfrak{p}}{ }^{+}$with $\|H\|=1$ and put $A_{\mathfrak{p}}{ }^{+}=\exp \mathfrak{a}_{\mathfrak{p}}{ }^{+}$.

(1) This means that $V$ is a left $K$-module under $\mu_{1}$ and a right $K$-module under $\mu_{2}$. Moreover, the operations of $K$ on the left, commute with those on the right. 
LEMMA 43. We keep to the notation of Lemma 42. Then the following three conditions are mutually equivalent.
1) $\nu=0$.
2) $\lim _{t \rightarrow+\infty} e^{t \mathfrak{Q}(H)} \phi(h \exp t H)=0$ for $H \in S^{+}$and $h \in A_{\mathfrak{p}}{ }^{+}$.
3) $\phi \in C(G) \otimes V$.

It is convenient to prove the above two lemmas together. We shall call $\nu$ the index of $\phi$. The uniqueness of $v$ is obvious from its definition. For the rest we use induction on $\operatorname{dim} \mathfrak{g}$. If $\mathfrak{p}=\{0\}, G$ is compact and all our assertions are true trivially with $\boldsymbol{v}=0$. So we may suppose that $\operatorname{dim} \mathfrak{p} \geqslant 1$. First assume that $\mathfrak{c}_{\mathfrak{p}} \neq\{0\}$.

Fix an element $H_{0} \in \mathfrak{c}_{\mathfrak{p}}$ with $\left\|H_{0}\right\|=1$ and let $\mathfrak{g}_{1}=\mathfrak{l}+\mathfrak{p}_{1}$ where $\mathfrak{p}_{1}$ is the orthogonal complement (see $\S 7$ ) of $\mathbf{R} H_{0}$ in $\mathfrak{p}$. Then $\mathfrak{g}_{1}$ is an ideal in $\mathfrak{g}$. Let $G_{1}$ be the analytic subgroup of $G$ corresponding to $\mathfrak{g}_{1}$. Then the mapping $(t, y) \rightarrow \exp t H_{0} \cdot y\left(t \in \mathbf{R}, y \in G_{1}\right)$ defines an analytic diffeomorphism of $\mathbf{R} \times G_{1}$ onto $G$. Put $\phi(t: y)=\phi\left(\exp t H_{0} \cdot y\right)$. Since $H_{0}$ lies in the center of $\mathfrak{g}$, we can, in view of condition 1) of Lemma 42, choose complex numbers $c_{i}$ $(0 \leqslant i \leqslant m)$ such that $c_{0}=1$ and

Therefore it is clear that

$$
\sum_{0 \leqslant i \leqslant m} c_{i} H_{0}^{m-i} \phi=0
$$

$$
\phi(t: y)=\sum_{1 \leqslant i \leqslant p} e^{(-1)^{\frac{1}{2} \lambda_{i} t}} \phi_{i}(t: y) \quad\left(t \in \mathbf{R}, y \in G_{1}\right)
$$

where $\lambda_{1}, \ldots, \lambda_{p}$ are distinct complex numbers,

$$
\phi_{i}(t: y)=\sum_{0 \leqslant j \leqslant d} t^{j} \phi_{i j}(y) \quad(1 \leqslant i \leqslant p)
$$

and $\phi_{i j}$ are (1) $C^{\infty}$ functions from $G_{1}$ to $V$. We may assume that $\phi_{i} \neq 0$. Then it follows (see [4 (j), § 15]) from condition 2 ) of Lemma 42 that $\lambda_{1}, \ldots, \lambda_{p}$ are all real. Now $K \subset G_{1}$ and $\operatorname{dim} G_{1}<\operatorname{dim} G$. Therefore it is easy to see that, if $\phi_{i j} \neq 0$, Lemmas 42 and 43 are applicable to $\phi_{i j}$ by induction hypothesis. Let $d y$ denote the Haar measure on $G_{1}$ and $\nu_{i j}$ the index of the function $\phi_{i j}$ on $G_{1}$. Moreover we put $v_{i j}=-\infty$ if $\phi_{i j}=0$. Now let

$$
\nu=1+\max _{i, j}\left(2 j+v_{i j}\right) \text {. }
$$

We shall prove that $\nu$ is the index of $\phi$.

We may obviously assume that $d x=d t d y$. Then for $T \geqslant 0$,

(1) Here we make use of the fact that the functions $f_{i j}(t)=t^{j} e^{(-1)^{\frac{1}{2}} \lambda_{i} t}(1 \leqslant i \leqslant p, j \geqslant 0)$ are linearly independent over $\mathbf{C}$. 


$$
\|\phi\|_{T} \leqslant\left\{\int_{0}^{T} d t \int_{\sigma(y) \leqslant T}|\phi(t: y)|^{2} d y\right\}^{t} \leqslant \sum_{i, j}\left\{\int_{0}^{T} t^{2 j} d t \int_{\sigma(y) \leqslant T}\left|\phi_{i j}(y)\right|^{2} d y\right\}^{t}
$$

by the triangle inequality. Now fix $i, j$ such that $\phi_{i j} \neq 0$. Then by the definition of $\nu_{i j}$, we can choose a number $b_{i j}>0$ such that

$$
\int_{\sigma(y) \leqslant T}\left|\phi_{i j}(y)\right|^{2} d y \leqslant b_{i j}(1+T)^{v_{i j}}
$$

for all $T \geqslant 0$. Therefore since $\nu \geqslant 1+2 j+v_{i j}$, it is clear that

$$
\underset{T \rightarrow \infty}{\lim \sup T^{-p / 2}\|\phi\|_{T}<\infty} \text {. }
$$

On the other hand, in order to show that

$$
\liminf _{T \rightarrow \infty} T^{-v / 2}\|\phi\|_{T}>0,
$$

it would be sufficient to obtain the following result.

Lемм А 44. Fix $\delta>0$. Then

$$
\underset{T \rightarrow \infty}{\liminf } T^{-v} \int_{\substack{t^{2}+\sigma(y)^{2} \leqslant T^{2} \\ \sigma(y) \leqslant \delta t}}|\phi(t: y)|^{2} d t d y>0 .
$$

We may obviously assume that $\delta \leqslant 1$. For $T \geqslant 0$, let $J(T)$ denote the interval $T / 2 \leqslant t \leqslant T / \sqrt{2}$ and $G_{1}(T)$ the set of all points $y \in G_{1}$ with $\sigma(y) \leqslant T$. If $t \in J(T)$ and $y \in G_{1}(\delta T / 2)$, it is obvious that $t^{2}+\sigma(y)^{2} \leqslant T^{2}$ and $\sigma(y) \leqslant \delta t$. Hence it would be enough to show that

$$
\liminf _{T \rightarrow \infty} T^{-\vartheta} I(T)>0
$$

where

$$
I(T)=\int_{J(T)} d t \int_{G_{1}(\delta T / 2)}|\phi(t: y)|^{2} d y
$$

Let $\left\langle v_{1}, v_{2}\right\rangle\left(v_{1}, v_{2} \in V\right)$ denote the scalar product in $V$ and put

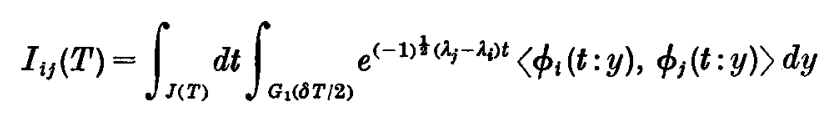

and $I_{i}(T)=I_{i i}(T)(1 \leqslant i, j \leqslant p)$. Then it is clear that $\left({ }^{1}\right)$

$$
I(T)=\sum_{1 \leqslant i \leqslant p} I_{i}(T)+2 \Re \sum_{1 \leqslant i \leqslant j \leqslant p} I_{i j}(T) .
$$

Fix $m, n(0 \leqslant m, n \leqslant d)$ and put

(1) $\Re c$ denotes the real part of a complex number $c$. 


$$
I_{i m, j n}(T)=\int_{J(T)} d t \int_{G_{1}(\delta T / 2)} e^{(-1)^{\frac{1}{2}}\left(\lambda_{j}-\lambda_{i}\right) t} t^{m+n}\left\langle\phi_{i m}(y), \phi_{j n}(y)\right\rangle d y
$$

for $1 \leqslant i, j \leqslant p$. Then if $i \neq j$, there exists a constant $a(i m, j n) \geqslant 0$ such that

$$
\left|\int_{J(T)} t^{m+n} e^{(-1)^{\frac{1}{2}}\left(\lambda_{j}-\lambda_{i}\right) t} d t\right| \leqslant a(i m, j n)(1+T)^{m+n} \quad(T \geqslant 0)
$$

(This follows by integrating by parts and using induction on $m+n$.) Hence it is clear that

$$
\left|I_{i m, j n}(T)\right| \leqslant a(i m, j n)(1+T)^{m+n}\left\|\phi_{i m}\right\|_{\delta T / 2}\left\|\phi_{j n}\right\|_{\delta T / 2},
$$

where

$$
\left\|\phi_{k l}\right\|_{t}^{2}=\int_{G_{1}(t)}\left|\phi_{k l}(y)\right|^{2} d y \quad(t \geqslant 0)
$$

for $1 \leqslant k \leqslant p, 1 \leqslant l \leqslant d$. Therefore if $\phi_{i m} \neq 0, \phi_{s n} \neq 0$, we get

$$
\left|I_{i m, j n}(T)\right| \leqslant b(i m, j n)(1+T)^{m+n+\left(v_{i m}+v_{j n}\right) / 2} \leqslant b(i m, j n)(1+T)^{y-1} \quad(T \geqslant 0),
$$

where $b(i m, j n)$ is a positive number independent of $T$. Therefore it would be enough to verify that

$$
\liminf _{T \rightarrow \infty} T^{-\nu} \sum_{1 \leqslant i \leqslant p} I_{i}(T)>0
$$

Fix $i$ such that $\nu_{i j}+2 j+1=\nu$ for some $j$. Then it would suffice to show that

$$
\liminf _{T \rightarrow \infty} T^{-v} I_{i}(T)>0 .
$$

Let $Q$ be the set of all $j(0 \leqslant j \leqslant d)$ such that $\nu_{i j}+2 j+1=\nu$. Then if $j \notin Q$, we have $v_{i j}+2 j+1<\nu$. Hence

$$
T^{-v} \int_{J(T)} t^{2 j} d t \int_{G_{1}(\delta T / 2)}\left|\phi_{i j}(y)\right|^{2} d y \leqslant T^{v_{i j}+2 j+1-v} T^{-v_{i j}}\left\|\phi_{i j}\right\|_{T}^{2} \rightarrow 0
$$

as $T \rightarrow \infty$. Put

$$
\phi_{i}{ }^{0}(t: y)=\sum_{j \in Q} t^{j} \phi_{i j}(y) \quad \text { and } \quad I_{i}{ }^{0}(T)=\int_{J(T)} d t \int_{G_{2}(\delta T / 2)}\left|\phi_{i}{ }^{0}(t: y)\right|^{2} d y .
$$

Then it would obviously be enough to prove that

$$
\underset{T \rightarrow \infty}{\liminf } T^{-\nu} I_{i}^{0}(T)>0
$$

Now fix $\varepsilon(0<\varepsilon \leqslant \delta / 2)$ and put 


$$
I(\varepsilon: T)=\int_{J(T)} d t \int_{G_{1}(\varepsilon T)}\left|\phi_{i}^{0}(t: y)\right|^{2} d y .
$$

Then $I(\varepsilon: T) \leqslant I_{i}{ }^{0}(T)$ and so it is sufficient to verify that

$$
\liminf _{T \rightarrow \infty} T^{-\nu} I(\varepsilon: T)>0 .
$$

Put

$$
I_{m n}(\varepsilon: T)=\int_{J(T)} d t \int_{G_{1}(e T)} t^{m+n}\left\langle\phi_{i m}(y), \phi_{i n}(y)\right\rangle d y
$$

and

$$
I_{m}(\varepsilon: T)=I_{m m}(\varepsilon: T) \quad(m, n \in Q)
$$

Then

$$
I(\varepsilon: T)=\sum_{j \in Q} I_{j}(\varepsilon: T)+2 \Re \sum_{\substack{m, n \in Q \\ m<n}} I_{m n}(\varepsilon: T) .
$$

Moreover, since $\phi_{i j} \neq 0$ for $j \in Q$, we can, by induction hypothesis, choose positive numbers $a, b$ such that

and

$$
\left\|\phi_{i j}\right\|_{T} \leqslant b(1+T)^{v_{i j} / 2} \quad(T \geqslant 0, j \in Q)
$$

$$
\left\|\phi_{i j}\right\|_{T} \geqslant a T^{v_{i j} / 2} \quad(j \in Q)
$$

for $T$ sufficiently large. Then

$$
\left|I_{m n}(\varepsilon: T)\right| \leqslant b^{2} T^{m+n+1}(1+\varepsilon T)^{\left(v_{i m}+v_{i n}\right) / 2} \quad(m, n \in Q) .
$$

Hence

$$
I(\varepsilon: T) \geqslant \sum_{j \in Q} c_{j} T^{2 j+1}(\varepsilon T)^{v_{i j}}-2 \sum_{\substack{m, n \in Q \\ m<n}} b^{2} T^{m+n+1}(1+\varepsilon T)^{\left(v_{i m}+v_{i n}\right) / 2},
$$

for large $T$, where

$$
c_{j}=a^{2}\left(2^{-\left(j+\frac{1}{2}\right)}-2^{-(2 j+1 j}\right) /(2 j+1)>0 .
$$

Let $k=\max _{j \in Q} j$. Then $v_{i j}-v_{i k}=2(k-j)$ and therefore

$$
I(\varepsilon: T) \geqslant T^{w} \varepsilon^{\nu_{i k}}\left\{\sum_{j \in Q} c_{j} \varepsilon^{2(k-\jmath)}-2 \sum_{\substack{m, n \in Q \\ m>n}} b^{2} \varepsilon^{2 k-m-n}\left(\mathbf{1}+(\varepsilon T)^{-1}\right)^{\left(v_{i m}+v_{i n}\right) / 2}\right\} .
$$

This shows that

$$
\liminf _{T \rightarrow \infty} T^{-v} I(\varepsilon: T) \geqslant \varepsilon^{v_{i k}}\left\{c_{k}+\sum_{j \in Q^{*}} c_{j} \varepsilon^{2(k-j)}-2 \sum_{\substack{m, n \in Q \\ m>n}} b^{2} \varepsilon^{2 k-m-n}\right\}
$$

where $Q^{\prime}$ is the set of all $j \neq k$ in $Q$. Now $c_{k}>0$ and $2 k-m-n \geqslant 1$ since $m<n$. Hence

$$
\lim _{T \rightarrow \infty} \inf ^{-v} I(\varepsilon: T)>0
$$

if $\varepsilon$ is sufficiently small. This proves Lemma 44 . 
If $l=\operatorname{dim} \mathfrak{c}_{\mathfrak{p}}$, it is obvious thas $\operatorname{dim}\left(\mathfrak{c}_{\mathfrak{p}} \cap \mathfrak{g}_{1}\right)=l-1$. Hence, by induction hypothesis, $v_{i j} \geqslant l-1$ whenever $\phi_{i j} \neq 0$. Therefore

$$
v=\max _{i, j}\left(v_{i j}+2 j+1\right) \geqslant l=\operatorname{dim} \mathfrak{c}_{\mathfrak{p}}
$$

Now we come to the last assertion of Lemma 42 . By the triangle inequality,

$$
\left\{\|\phi\|_{T_{2}}-\|\phi\|_{T_{1}}^{2}\right\}^{\frac{1}{2}} \leqslant \sum_{1 \leqslant i \leqslant p}\left\{\left\|\phi_{i}\right\|_{T_{2}}{ }^{2}-\left\|\phi_{i}\right\|_{T_{1}}{ }^{2}\right\}^{\frac{1}{2}}
$$

for $T_{2} \geqslant T_{1} \geqslant 0$. Here

$$
\phi_{i}\left(\exp t H_{0} \cdot y\right)=\phi_{i}(t: y)=e^{(-1)^{\frac{1}{2}} \lambda_{i} t} \sum_{0 \leqslant j \leqslant d} t^{j} \phi_{i j}(y) \quad\left(t \in \mathbf{R}, y \in G_{\mathbf{1}}\right)
$$

as before. Put $v_{i}=1+\max _{j}\left(2 j+v_{i j}\right)$. Then $v=\max _{i} \boldsymbol{v}_{i}$ and so it would clearly be enough to consider the case when $p=1$. Hence we may assume that

$$
\phi(t: y)=e^{(-1)^{\frac{1}{2} \lambda t}} \sum_{0 \leqslant j \leqslant d} t^{j} \phi_{j}(y)
$$

where $\lambda$ is real. Then again, by the triangle inequality, we have

$$
\left\{\|\phi\|_{T_{2}^{2}}^{2}-\|\phi\|_{T_{1}}^{2}\right\}^{\frac{1}{2}} \leqslant \sum_{0 \leqslant j \leqslant d}\left\{\int_{T_{1^{2} \leqslant t^{2}+\sigma(y)^{3} \leqslant T_{2}^{2}}} t^{2 j}\left|\phi_{j}(y)\right|^{2} d t d y\right\}^{\frac{1}{2}}
$$

and so it would be sufficient to consider the case when

$$
\phi(t: y)=e^{(-1)^{\frac{1}{2} \lambda t}} t^{\prime} \phi_{1}(y)
$$

Then $y=2 j+1+\nu_{1}$ where $\nu_{1}$ is the index of the function $\phi_{1}$ on $G_{1}$.

Now suppose $0 \leqslant T_{1} \leqslant T_{2}$. Then

where

$$
\|\phi\|_{T_{2}}^{2}-\|\phi\|_{T_{1}}^{2}=2 I_{1}\left(T_{1}: T_{2}\right)+2 I_{2}\left(T_{1}: T_{2}\right),
$$

$$
I_{1}\left(T_{1}: T_{2}\right)=\int_{0}^{T_{1}} t^{2 j} d t \int_{\left(T_{1}^{2}-t^{2}\right) \leqslant \sigma(y)^{2} \leqslant\left(T_{\left.2^{2}-t^{2}\right)}\right.}\left|\phi_{1}(y)\right|^{2} d y
$$

and

$$
I_{2}\left(T_{1}: T_{2}\right)=\int_{T_{1}}^{T_{3}} t^{2 j} d t \int_{\sigma(y)^{2} \leqslant T_{2^{2}-t^{2}}}\left|\phi_{1}(y)\right|^{2} d y
$$

Fix $\varepsilon>0$ and select a small number $\varepsilon_{1}>0$. Then, by induction hypothesis, we can choose $t_{0} \geqslant 1$ and $\delta_{1}\left(0<\delta_{1}<1\right)$ with the property that

$$
\int_{t_{1} \leqslant \sigma(y) \leqslant t_{2}}\left|\phi_{1}(y)\right|^{2} d y \leqslant \varepsilon_{1} t_{1}^{\nu_{1}}
$$


if $t_{0} \leqslant t_{1} \leqslant t_{2} \leqslant\left(1+\delta_{1}\right) t_{1}$. Fix a small positive number $\delta(0<\delta<1)$ and put $\gamma=1-\delta^{t}$. Then if $0 \leqslant t \leqslant \gamma T_{1}$ and $0<T_{1} \leqslant T_{2} \leqslant(1+\delta) T_{1}$, we have

$$
\begin{aligned}
& \left(T_{2}^{2}-t^{2}\right)^{\frac{1}{2}}\left(T_{1}^{2}-t^{2}\right)^{-\frac{1}{2}}-1 \leqslant\left(T_{2}^{2}-t^{2}\right)\left(T_{1}^{2}-t^{2}\right)^{-1}-1 \\
& =\left(T_{2}^{2}-T_{1}^{2}\right)\left(T_{1}^{2}-t^{2}\right)^{-1} \leqslant\left\{(1+\delta)^{2}-1\right\}\left(1-\gamma^{2}\right)^{-1} \leqslant 3 \delta^{2} \leqslant \delta_{1},
\end{aligned}
$$

provided $\delta$ is sufficiently small. Moreover,

$$
\left(T_{1}^{2}-t^{2}\right)^{\frac{1}{2}} \geqslant T_{1}\left(1-\gamma^{2}\right)^{\frac{1}{2}} \geqslant T_{1} \delta^{\frac{1}{2}} \geqslant t_{0} .
$$

if $T_{1} \geqslant T_{0}=\delta^{-\frac{1}{t}} t_{0}$. Therefore if $T_{0} \leqslant T_{1} \leqslant T_{2} \leqslant(1+\delta) T_{1}$ and $0 \leqslant t \leqslant \gamma T_{1}$, we have

$$
\int_{\left(T_{1}^{2}-t^{2}\right) \leqslant \sigma(y)^{2} \leqslant\left(T_{2}^{2}-t^{2}\right)}\left|\phi_{1}(y)\right|^{2} d y \leqslant \varepsilon_{1}\left(T_{1}^{2}-t^{2}\right)^{p_{2} / 2} .
$$

But then since $\nu=v_{1}+2 j+1$, we conclude that

$$
\int_{0}^{\gamma T_{1}} t^{2 j} d t \int_{\left(T_{1}^{2}-t^{2}\right) \leqslant \sigma(y)^{2} \leqslant\left(T_{\left.2^{2}-t^{2}\right)}\right.}\left|\phi_{1}(y)\right|^{2} d y \leqslant \varepsilon_{1} T_{1}^{\nu}
$$

On the other hand, we can select $b>0$ such that

$$
\int_{G_{1}(t)}\left|\phi_{1}(y)\right|^{2} d y \leqslant b(1+t)^{\nu_{1}}
$$

for $t \geqslant 0$. Therefore if $T_{0} \leqslant T_{1} \leqslant T_{2} \leqslant(1+\delta) T_{1}$, we have

$$
\int_{\gamma T_{1}}^{T_{1}} t^{2 j} d t \int_{\left(T_{1}^{2}-t^{2}\right) \leqslant \sigma(y)^{2} \leqslant\left(T_{3}^{2}-t^{3}\right)}\left|\phi_{1}(y)\right|^{2} d y \leqslant b \int_{\gamma T_{1}}^{T_{1}} t^{2 j}\left(1+T_{2}\right)^{\nu_{1}} d t \leqslant 3^{\nu_{1}} b T_{1}^{\nu} \delta^{\frac{1}{2}}
$$

since $1+T_{2} \leqslant 1+(1+\delta) T_{1} \leqslant 3 T_{1}$. This proves that

$$
I_{1}\left(T_{1}: T_{2}\right) \leqslant T_{1}^{\nu}\left(3^{\nu_{2}} b \delta^{\frac{1}{2}}+\varepsilon_{1}\right)
$$

if $T_{0} \leqslant T_{1} \leqslant T_{2} \leqslant(1+\delta) T_{1}$. On the other hand, it is obvious that

$$
I_{2}\left(T_{1}: T_{2}\right) \leqslant T_{2}^{2 j}\left(T_{2}-T_{1}\right) b\left(1+T_{2}\right)^{\nu_{1}} \leqslant 3^{v} b T_{1}^{v} \delta
$$

Therefore

$$
\|\phi\|_{T_{2}}{ }^{2}-\|\phi\|_{T_{1}}{ }^{2}=2 I_{1}\left(T_{1}: T_{2}\right)+2 I_{2}\left(T_{1}: T_{2}\right) \leqslant 2\left(\varepsilon_{1}+3^{\nu_{1}} b \delta^{\frac{1}{2}}+3^{v} b \delta\right) T_{1}{ }^{v} \leqslant \varepsilon T_{1}{ }^{\nu},
$$

if $\varepsilon_{1}$ and $\delta$ are chosen sufficiently small. This completes the proof of all the statements of Lemma 42 in case $\operatorname{dim} \mathfrak{c}_{\mathfrak{p}} \geqslant 1$. 
Now we come to Lemma 43. Since $\nu \geqslant \operatorname{dim} \mathfrak{c}_{\mathfrak{p}} \geqslant 1$, condition 1 ) of Lemma 43 cannot be fulfilled in the present case. Since it is obvious that 3 ) implies 2), it would be enough to verify that 2 ) is never satisfied in our case. But since $\varrho\left(H_{0}\right)=0$, it is clear from [4 $\left.(\mathrm{j}), \S 15\right]$ that 2) implies that $\phi=0$, giving a contradiction. This proves Lemma 43 when $\operatorname{dim} \mathfrak{c}_{\mathfrak{p}} \geqslant 1$.

We state the following result, which has been proved above, as a lemma for future reference.

L кмм 45. Given $\varepsilon>0$, we can choose $\delta>0$ and $T_{0}>0$ such that

$$
\int_{T_{1}^{2} \leqslant t^{2}+\sigma(y)^{2} \leqslant T_{2}^{2}}|\phi(t: y)|^{2} d t d y \leqslant \varepsilon T_{1}^{\prime}
$$

for $T_{0} \leqslant T_{1} \leqslant T_{2} \leqslant(1+\delta) T_{1}$

\section{\$ 27. The differential equation for $\Phi$}

In order to complete the proofs of Lemmas 42 and 43 , we may now assume that $\mathfrak{c}_{\mathfrak{p}}=\{0\}$ and $\mathfrak{p} \neq\{0\}$. Fix an element $H_{0} \in S^{+}$and let $\mathfrak{m}$ be the centralizer of $H_{0}$ in $g$ and $\mathfrak{I}$ the centralizer of $\mathfrak{m}$ in $\mathfrak{a}_{\mathfrak{p}}$. Since $\mathfrak{a}_{\mathfrak{p}} \subset \mathfrak{m}$ and $\mathfrak{a}_{\mathfrak{p}}$ is maximal abelian in $\mathfrak{p}, \mathfrak{l}$ is also the centralizer of $\mathfrak{m}$ in $\mathfrak{p}$. Moreover, $\operatorname{dim} \mathfrak{m}<\operatorname{dim} \mathfrak{g}$.

Put $\mathfrak{M}=\subseteq\left(\mathfrak{m}_{c}\right)$ and let $\mathbb{B}_{\mathfrak{m}}$ denote the center of $\mathfrak{M}$ and $\mu_{0}$ the homomorphism (see [4(o), $\S 12]$ ) of 8 into $B_{1}=B_{\mathfrak{m}}$. Define $\mathfrak{U}_{1}=8_{1} \mu_{0}(\mathfrak{U})$ where $\mathfrak{U}$ is the ideal consisting of all $u \in 8$ such that $u \phi=0$. Then it follows from condition 1) of Lemma 42 and [4 (o), Lemma 21] that $\operatorname{dim} 8 / \mathfrak{A l}$ and $\operatorname{dim} 81 / \mathfrak{U}_{1}$ are both finite. Let $\zeta \rightarrow \zeta^{*}$ denote the natural projection of $B_{1}$ on $B_{1}{ }^{*}=B_{1} / \mathfrak{H}_{1}$. We regard $B_{1}{ }^{*}$ as a $B_{1}$-module in the usual way so that $z \zeta^{*}=(z \zeta)^{*}$ $\left(z, \zeta \in B_{1}\right)$. Let $\delta_{1}^{* *}$ be the vector space dual to $\delta_{1}^{*}$. Then since $\delta_{1}$ is abelian, $\delta_{1}^{* *}$ is also a left $8_{1}$-module by duality. Put $\mathbf{V}=V \otimes B_{1}{ }^{* *}$ and let $\Gamma$ denote the representation of $B_{1}$ on $\mathbf{V}$ defined by

$$
\boldsymbol{\Gamma}(z)\left(v \otimes \zeta^{* *}\right)=v \otimes z \zeta^{* *} \quad\left(z \in \oiint_{1}, v \in V, \zeta^{* *} \in 8_{1}^{* *}\right)
$$

Moreover, by making $K$ act trivially on $8_{1}{ }^{* *}$, we can regard $\mathrm{V}$ as a double $K$-module. Note that $\boldsymbol{\Gamma}(z)\left(z \in 8_{1}\right)$ commutes with the operations of $K$ on $\mathbf{V}$.

Fix elements $\eta_{1}=1, \eta_{2}, \ldots, \eta_{p}$ in $8_{1}$ such that $\eta_{1}{ }^{*}, \ldots, \eta_{p}{ }^{*}$ is a base for $81^{*}$. Let $\eta_{i}{ }^{* *}$ $(1 \leqslant i \leqslant p)$ be the dual base for $8_{1}{ }^{* *}$. We regard $8_{1}{ }^{* *}$ as a Hilbert space with $\left(\eta_{1}{ }^{* *}, \ldots, \eta_{p}{ }^{* *}\right)$ as an orthonormal base. Then $V$ also becomes a Hilbert space.

Let $\Sigma$ be the set of all positive roots of $\left(\mathfrak{g}, \mathfrak{a}_{\mathfrak{p}}\right), \Sigma_{1}$ the subset of those $\alpha \in \Sigma$ which vanish identically on $\mathfrak{l}$ and $\Sigma_{2}$ the complement of $\Sigma_{1}$ in $\Sigma$. For any $\alpha \in \Sigma$, let $g_{\alpha}$ denote the set of all $X \in \mathfrak{g}$ such that $[H, X]=\alpha(H) X$ for $H \epsilon_{a_{\mathfrak{p}}}$. Put 


$$
\mathfrak{n}_{i}=\sum_{\alpha \in \Sigma_{i}} \mathfrak{g}_{\alpha},(i=1,2)
$$

and $\mathfrak{n}=\mathfrak{n}_{1}+\mathfrak{n}_{2}$. Let $M$ be the analytic subgroup of $G$ corresponding to $\mathfrak{m}$. Then $M$ normalizes $\mathfrak{t}_{\mathbf{2}}$. Put

$$
d(m)=\left|\operatorname{det}(\operatorname{Ad}(m))_{\mathfrak{n}_{\mathrm{a}}}\right|^{\frac{1}{2}} \quad(m \in M)
$$

and $\zeta^{\prime}=d^{-1} \zeta$ od for $\zeta \in 8_{1}$. It is easy to verify (see the Appendix, $\S 45$ ) that $\zeta \rightarrow \zeta^{\prime}$ is an automorphism of $8_{1}$. Now define

$$
\Phi(m)=\sum_{1 \leqslant i \leqslant p} \phi_{i}(m) \otimes \eta_{i}^{* *} \quad(m \in M)
$$

where

$$
\phi_{i}(m)=d(m) \phi\left(m ; \eta_{i}{ }^{\prime}\right)
$$

Then it is obvious that $\Phi$ is a spherical function from $M$ to $\mathrm{V}$ corresponding to the double representation of $K_{1}=M \cap K$ on $V$ which we denote by $\mu=\left(\mu_{1}, \mu_{2}\right)$.

For any $\zeta \in B_{1}$, there exist unique complex numbers $c_{i j}$ such that

$$
u_{i}(\zeta)=\zeta \eta_{i}-\sum_{1 \leqslant j \leqslant p} c_{i j} \eta_{j} \in \mathfrak{U}_{1} \quad(1 \leqslant i \leqslant p)
$$

Lwмма 46. Let $\zeta \in 8_{1}$. Then

$$
\Phi(m ; \zeta)-\boldsymbol{\Gamma}(\zeta) \Phi(m)=\sum_{1 \leqslant i \leqslant p} d(m) \phi\left(m ; u_{i}(\zeta)^{\prime}\right) \otimes \eta_{i}{ }^{* *}
$$

for $m \in M$.

Fix $\zeta$ and define $c_{i j}$ as above. Then

$$
\Phi(m ; \zeta)=\sum_{i} \phi_{i}(m ; \zeta) \otimes \eta_{i}^{* *} \quad \text { and } \quad \zeta^{\prime} \eta_{i}^{\prime}=\sum_{j} c_{i j} \eta_{j}^{\prime}+u_{i}^{\prime}
$$

where $u_{i}=u_{i}(\zeta)$. Therefore since

our assertion is obvious.

$$
\phi_{i}(m ; \zeta)=d(m) \phi\left(m ; \zeta^{\prime} \eta_{i}^{\prime}\right)
$$

Put $\quad \Psi_{\zeta}(m)=\sum_{1 \leqslant i \leqslant p} d(m) \phi\left(m ; u_{i}(\zeta)^{\prime}\right) \otimes \eta_{i}^{* *} \quad\left(\zeta \in \AA_{1}, m \in M\right)$.

Then the following corollary is merely a restatement of the above lemma.

Corollary 1. $\Phi(m ; \zeta)=\Gamma(\zeta) \Phi(m)+\Psi_{\zeta}(m)$ for $m \in M$ and $\zeta \in \oiint_{1}$.

Now $\mathfrak{l}$ lies in the center of $\mathfrak{m}$. Hence if $H \in \mathfrak{l}$, we conclude from the above corollary that

$$
d\left(e^{-t \Gamma(H)} \Phi(m \exp t H)\right) / d t=e^{-t \Gamma(H)} \Psi_{H}(m \exp t H)
$$

for $t \in \mathbf{R}$ and $m \in M$. Therefore the following result is now obvious. 
CoRollaky 2. $\Phi(m \exp T H)=e^{T \Gamma(H)} \Phi(m)+\int_{0}^{T} e^{(T-t) \Gamma(H)} \Psi_{H}(m \exp t H) d t$ for $m \in M$, $H \in \mathfrak{l}$ and $T \in \mathbf{R}$.

\section{\$ 28. Some estimates for $\Phi$ and $\Psi_{\zeta}$}

Let $\Xi_{M}=\Xi_{1}$ be the function on $M$ which corresponds to $\Xi$ when $(G, K)$ is replaced by $\left(M, K_{1}\right)$.

Lемм 4 4. There exist numbers $c_{0}, r_{0} \geqslant 0$ such that

for $H \in \mathfrak{a}_{\mathfrak{p}}{ }^{+}$.

$$
d(\exp H) \Xi(\exp H) \leqslant c_{0} \Xi_{1}(\exp H)(1+\|H\|)^{r_{0}}
$$

We shall give a proof of this lemma in $\$ \mathbf{4 5}$.

Put $M^{+}=K_{1} A_{\mathfrak{p}}+K_{1}$. Since $K_{1}$ lies in the kernel of the homomorphism $d$, the following corollary is obvious.

CoRollaRY. $d(m) \Xi(m) \leqslant c_{0} \Xi_{1}(m)(1+\sigma(m))^{\gamma_{0}} \quad\left(m \in M^{+}\right)$.

Fix $r \geqslant 0$ as in condition 2) of Lemma 42 .

LEM M 48. Given $g_{1}, g_{2} \in\left(\mathfrak{S}\right.$, we can choose a number $c\left(g_{1}, g_{2}\right) \geqslant 0$ such that

$$
\left|\phi\left(g_{1} ; x ; g_{2}\right)\right| \leqslant c\left(g_{1}, g_{2}\right) \Xi(x)(1+\sigma(x))^{r} \quad(x \in G) .
$$

In view of Lemma 17, it is enough to consider the case $g_{1}=1$. By Theorem 1 , we can choose $\alpha \in C_{c}^{\infty}(G)$ such that $\phi * \alpha=\phi$. Then $g \phi=\phi *(g \alpha)(g \in(B)$ and so our assertion follows immediately from Lemma 10 and [4 (q), Lemma 32].

Put $r_{1}=r+r_{0}$.

CoROLLARY. For any $v \in \mathfrak{M}$, we can choose a number $c(v) \geqslant 0$ such that

$$
|\Phi(m ; v)| \leqslant c(v) \Xi_{\mathbf{1}}(m)(1+\sigma(m))^{r_{\mathbf{1}}} \quad\left(m \in M^{+}\right) .
$$

This is obvious from Lemma 48 and the corollary of Lemma 47.

For $\alpha \in \Sigma$, define $\mathfrak{g}_{\alpha}$ as in $\$ 27$.

LEMMA 49. Fix $g \in\left(S, \alpha \in \Sigma\right.$ and $X \in \mathfrak{g}_{\alpha}$. Then we can choose $c_{1} \geqslant 0$ such that

for $h \in A_{\mathfrak{p}}{ }^{+}$.

$$
|d(h) \phi(h ; \theta(X) g)| \leqslant c_{1} e^{-\alpha(\log h)} \Xi_{1}(h)(1+\sigma(h))^{r_{1}}
$$

Since $\phi(h ; \theta(X) g)=e^{-\alpha(\log h)} \phi(\theta(X) ; h ; g)$, this follows from Lemmas 47 and 48 .

Corollar Y. Fix $\zeta \in B_{1}$ and $v \in \mathfrak{M}$. Then we can choose $c \geqslant 0$ such that

$$
\left|\Psi_{\zeta}(m \exp H ; v)\right| \leqslant c e^{-\beta(H)} \Xi_{1}(m)(1+\sigma(m))^{\gamma_{1}}(1+\|H\|)^{r_{1}}
$$


for $H \in \mathfrak{l}^{+}=\mathfrak{l} \cap \mathfrak{a}_{\mathfrak{p}}+$ and $m \in M^{+}$. Here

$$
\beta(H)=\min _{\alpha \in \Sigma} \alpha(H) .
$$

We know that (see the Appendix, §45)

$$
z-\mu_{0}(z)^{\prime} \in \theta\left(\mathfrak{n}_{2}\right) \Theta
$$

Fix $g \in\left(S, u \in \mathbb{U}\right.$ and put $g_{1}=-\left(u-\mu_{0}(u)^{\prime}\right) g \in \theta\left(\mathfrak{n}_{2}\right)(\mathfrak{S}$. Since $u \phi=0$, it is clear that

$$
\phi\left(m ; \mu_{0}(u)^{\prime} g\right)=\phi\left(m ; g_{1}\right)
$$

Define the automorphism $v \rightarrow v^{\prime}=d^{-1} v \circ d$ on $\mathfrak{M}$ as in the Appendix, $\S 45$. Fix $v$ and $\zeta$ as above. Then

$$
\Psi_{\zeta}^{*}(m ; v)=\sum_{1 \leqslant i \leqslant p} d(m) \phi\left(m ; v^{\prime} u_{i}(\zeta)^{\prime}\right) \otimes \eta_{i}^{* *}
$$

Since $u_{i}(\zeta) \in \mathbb{H}_{1} \subset Z_{1}, v$ commutes with $u_{i}(\zeta)$ and therefore

$$
v u_{i}(\zeta)=u_{i}(\zeta) v \in \mu_{0}(\mathfrak{U}) \mathfrak{M}
$$

Hence, in view of the remark above, we can choose $g_{i} \in \theta\left(\mathfrak{n}_{2}\right) \mathbb{S}$ such that

$$
\phi\left(m ; v^{\prime} u_{i}(\zeta)^{\prime}\right)=\phi\left(m ; g_{i}\right) \quad(m \in M)
$$

Since $M^{+}=K_{1} A_{\mathfrak{\downarrow}}{ }^{+} K_{1}$, our assertion now follows easily from Lemma 49 .

\section{$\$ 29$. The function $\Theta$}

Let $Q$ be the set of all eigenvalues of $\Gamma\left(H_{0}\right)$. Then

$$
\mathbf{V}=\sum_{\lambda \in Q} \mathbf{V}_{\lambda}
$$

where the sum is direct and $\nabla_{\lambda}$ is the subspace consisting of all $v \in V$ such that $\left(\Gamma\left(H_{0}\right)-\lambda\right)^{m} v=0$ for some integer $m \geqslant 0$. Let $E_{\lambda}$ denote the projection of $\mathbf{V}$ on $\mathbf{V}_{\lambda}$ corresponding to the above sum. We divide $Q$ into three disjoint sets $Q^{+}, Q^{0}$ and $Q^{-}$. An element $\lambda \in Q$ lies in $Q^{+}, Q^{0}$ or $Q^{-}$according as $\left(^{1}\right) \Re \lambda>0, \Re \lambda=0$ or $\Re \lambda<0$. Put

$$
E^{ \pm}=\sum_{\lambda \in Q^{ \pm}} E_{\lambda}, E^{0}=\sum_{\lambda \in Q^{0}} E_{\lambda}
$$

and

$$
\mathbf{V}^{ \pm}=E^{ \pm} \mathbf{V}, \mathbf{V}^{\mathbf{0}}=E^{0} \mathbf{V}
$$

Since $Z_{1}$ is abelian, $\Gamma(\zeta)\left(\zeta \in Z_{1}\right)$ commutes with the projections $E_{\lambda}$.

(1) $\Re_{c}$ denotes the real part of a complex number $c$. 
Put $Q^{\prime}=Q^{+} \cup Q^{-}$and $\operatorname{let}\left({ }^{1}\right)$

$$
4 \varepsilon_{0}=\min _{\lambda \in Q^{\prime}}|\Re \lambda| .
$$

Then $\varepsilon_{0}>0$. Put

$$
\beta(H)=\min _{\alpha \in \Sigma_{2}} \alpha(H) \quad(H \in \mathfrak{l})
$$

as before, so that $\beta\left(H_{0}\right)>0$. Fix a number $\varepsilon\left(0<\varepsilon \leqslant \varepsilon_{0}\right)$ and an open and relatively compact neighborhood $\Omega$ of $H_{0}$ in $\mathfrak{l}$. By selecting them sufficiently small, we can arrange that $\beta\left(H_{0}\right) \geqslant 5 \varepsilon$ and $\left({ }^{2}\right)$

for $H \in \Omega$.

$$
\beta(H) \geqslant 4 \varepsilon,\left|\boldsymbol{\Gamma}(H)-\boldsymbol{\Gamma}\left(H_{0}\right)\right|<\varepsilon / 2
$$

Leмma 50. We can choose a number $c \geqslant 0$ such that

$$
\left|e^{-t \boldsymbol{\Gamma}(H)} E^{+}\right|+\left|e^{t \mathbf{\Gamma}(H)} E^{-}\right| \leqslant c e^{-2 \varepsilon_{0} t} \quad(t \geqslant 0
$$

and

$$
\left|e^{t \mathbf{\Gamma}(H)} E^{0}\right| \leqslant c e^{\varepsilon|t|} \quad(t \in \mathbf{R})
$$

for $H \in \Omega$.

Fix $\lambda \in Q$. Then

$$
\boldsymbol{\Gamma}(H) E_{\lambda}=\left\{\lambda+\left(\boldsymbol{\Gamma}(H)-\boldsymbol{\Gamma}\left(H_{0}\right)\right)+\left(\boldsymbol{\Gamma}\left(H_{0}\right)-\lambda\right)\right\} E_{\lambda}
$$

and therefore

$$
\left|e^{t \mathbf{\Gamma}(H)} E_{\lambda}\right| \leqslant \exp \{t \Re \lambda+\varepsilon|t| / 2\}\left|e^{t\left(\mathbf{T}\left(H_{0}\right)-\lambda\right)} E_{\lambda}\right|
$$

for $H \in \Omega$ and $t \in \mathbf{R}$. Since $\left(\boldsymbol{\Gamma}\left(H_{0}\right)-\lambda\right) E_{\lambda}$ is nilpotent, our assertions follow without difficulty.

Put $\quad \Phi^{ \pm}(\mathrm{m})=E^{ \pm} \Phi(m), \Phi^{0}(m)=E^{0} \Phi(m) \quad(m \in M)$.

Then by Corollary 2 of Lemma 41,

$$
\Phi^{-}(m \exp T H)=e^{T \Gamma(H)} \Phi^{-}(m)+\int_{0}^{T} e^{(T-t) \Gamma(H)} E^{-} \Psi_{H}(m \exp t H) d t
$$

for $H \in \Omega$ and $T \geqslant 0$. Therefore if $v \in \mathfrak{M}$, we conclude from Lemma 50 that

$$
\left|\Phi^{-}(m \exp T H ; v)\right| \leqslant c e^{-2 \varepsilon_{0} T}\left|\Phi^{-}(m ; v)\right|+c \int_{0}^{T} e^{-2(T-t) \varepsilon_{0}}\left|\Psi_{H}(m \exp t H ; v)\right| d t .
$$

Put $f(t)=\Psi_{H}(m \exp t H ; v)$ for fixed $m, H, v$. Then

(1) We define $\varepsilon_{0}=1$, in case $Q^{\prime}$ is empty.

(2) If $T$ is a bounded linear operator on a Banach space $\mathfrak{B},|T|=\sup _{|b| \leqslant 1}|T b|(b \in \mathfrak{B})$, as usual. 5-662900. Acta mathematica. 116. Imprimé le 10 juin 1966. 


$$
\begin{aligned}
\int_{0}^{T} e^{-2(T-t) \varepsilon_{0}}|f(t)| d t & \leqslant e^{-T \varepsilon_{0}} \int_{0}^{T / 2}|f(t)| d t+\int_{T / 2}^{T}|f(t)| d t \\
& \leqslant e^{-T \varepsilon_{0}} \int_{0}^{\infty}|f(t)| d t+\int_{T / 2}^{\infty}|f(t)| d t
\end{aligned}
$$

On the other hand, since $\Psi_{H}$ depends linearly on $H$ and $\Omega$ is relatively compact, we have the following result from the corollary of Lemma 49 .

LEMMA 51. For a given $v \in \mathfrak{M}$, there exists a number $c(v) \geqslant 0$ such that

$$
\left|\Psi_{H}(m \exp t H ; v)\right| \leqslant c(v) e^{-3 \varepsilon t} \Xi_{1}(m)(1+\sigma(m))^{r_{1}}
$$

for $m \in M^{+}, H \in \Omega$ and $t \geqslant 0$.

Therefore in view of the corollary of Lemma 48, we obtain the following lemma.

LEMMA 52. For any $v \in \mathfrak{M}$, we can select a number $c^{-}(v) \geqslant 0$ such that

$$
\left|\Phi^{-}(m \exp T H ; v)\right| \leqslant c^{-}(v) e^{-\varepsilon T} \Xi_{1}(m)(1+\sigma(m))^{r_{1}}
$$

for $m \in M^{+}, H \in \Omega$ and $T \geqslant 0$.

Now we come to $\Phi^{+}$. Again, by Corollary 2 of Lemma 46, we have

$$
\Phi^{+}(m ; v)=e^{-T \Gamma(H)} \Phi^{+}(m \exp T H ; v)-\int_{0}^{T} e^{-t \boldsymbol{\Gamma}(H)} E^{+} \Psi_{H}(m \exp t H ; v) d t
$$

Fix $H \in \Omega, m \in M^{+}$and let $T$ tend to $+\infty$. Then it is clear from Lemmas 50 and 51 and the corollary of Lemma 48 , that

and therefore

$$
\Phi^{+}(m ; v)=-\int_{0}^{\infty} e^{-t \mathbf{\Gamma}(H)} E^{+} \Psi_{H}(m \exp t H ; v) d t
$$

$$
\Phi^{+}(m \exp T H ; v)=-\int_{T}^{\infty} e^{-(t-T) \Gamma(H)} E^{+} \Psi_{H}(m \exp t H ; v) d t
$$

In view of Lemmas 50 and 51 , this gives the following result.

Lемм а 53. For any $v \in \mathfrak{M}$, there exists a number $c^{+}(v) \geqslant 0$ such that

$$
\left|\Phi^{+}(m \exp T H ; v)\right| \leqslant c^{+}(v) e^{-\varepsilon T} \Xi_{1}(m)(1+\sigma(m))^{\tau_{1}}
$$

for $m \in M^{+}, H \in \Omega$ and $T \geqslant 0$.

We shall now consider $\Phi^{0}$. But first we need some preparation. 
LEMMA 54. Let $C$ be a compact subset of $M$. Then we can choose $T_{0} \geqslant 0$ such that $m \exp T H \in M^{+}$for $m \in C, H \in \Omega$ and $T \geqslant T_{0}$.

We may obviously assume that $K_{1} C K_{1}=C$. Let $C_{1}$ be the set of all $h \in C \cap A_{\mathfrak{p}}$ such that $\alpha(\log h) \geqslant 0$ for all $\alpha \in \Sigma_{1}$. Then $C_{1}$ is also compact and $C=K_{1} C_{1} K_{1}$. Now choose $T_{0} \geqslant 0$ such that

$$
\alpha(\log h)+4 \varepsilon T_{0} \geqslant 0
$$

for all $h \in C_{1}$ and $\alpha \in \Sigma_{2}$. Then it is clear that $C_{1} \exp T H \subset A_{\mathfrak{p}^{+}}$and therefore $C \exp T H \subset M^{+}$ for $T \geqslant T_{0}$ and $H \in \Omega$.

Now fix $v \in \mathfrak{M}$ and $H \in \Omega$. Then we conclude from Lemmas 50, 51 and 54 that the integral

$$
\int_{0}^{\infty}\left|e^{-t \Gamma(H)} E^{0} \Psi_{H}(m \exp t H ; v)\right| d t
$$

converges uniformly for $m \in C$. Put

$$
\Theta_{H}(m)=\Phi^{0}(m)+\int_{0}^{\infty} e^{-t \Gamma(H)} E^{0} \Psi_{H}(m \exp t H) d t \quad(m \in M) .
$$

Then it is clear that $\Theta_{H}$ is a $C^{\infty}$ function on $M$ and

$$
\Theta_{H}(m ; v)=\Phi^{0}(m ; v)+\int_{0}^{\infty} e^{-t \Gamma(H)} E^{0} \Psi_{H}(m \exp t H ; v) d t
$$

Moreover, it follows from Corollary 2 of Lemma 46 that

$$
\Theta_{H}(m ; v)=\lim _{T \rightarrow+\infty} e^{-T \Gamma(H)} \Phi^{0}(m \exp T H ; v) .
$$

So, in particular, the following result is obvious.

Lемма 55. $\Theta_{H}(m \exp t H)=e^{t \mathbf{\Gamma}(H)} \Theta_{H}(m)(m \in M, H \in \Omega, t \in \mathbf{R})$.

We now claim that $\Theta_{H}$ is actually independent of $H$. Fix $H_{1}, H_{2} \in \Omega$ and $m \in M$ and choose $T_{0} \geqslant 0$ such that $m \exp t H \in M^{+}$for $t \geqslant T_{0}$ and $H \in \Omega$. Put $m_{2}=m \exp T_{2} H_{2}\left(T_{2} \geqslant T_{0}\right)$. Then by Corollary 2 of Lemma 46 ,

$$
e^{-\Gamma\left(T_{1} H_{1}\right)} \Phi^{0}\left(m_{2} \exp T_{1} H_{1}\right)=\Phi^{0}\left(m_{2}\right)+\int_{0}^{T_{2}} e^{-t \Gamma\left(H_{1}\right)} E^{0} \Psi_{H_{1}}\left(m_{2} \exp t H_{1}\right) d t
$$

and therefore

$$
\begin{aligned}
e^{-\Gamma\left(T_{3} H_{1}+T_{2} H_{2}\right)} \Phi^{0}\left(m \exp \left(T_{1} H_{1}+T_{2} H_{2}\right)\right)-e^{-\Gamma\left(T_{2} H_{2}\right)} \Phi^{0}\left(m \exp T_{2} H_{2}\right) \\
\doteq \int_{0}^{T_{1}} e^{-\Gamma\left(t_{1} H_{1}+T_{2} H_{2}\right)} E^{0} \Psi_{H_{1}}\left(m \exp \left(t_{1} H_{1}+T_{2} H_{2}\right)\right) d t_{1}
\end{aligned}
$$


for $T_{1} \geqslant 0$. But it follows from Lemma 50 and the corollary of Lemma 49 that there exists a number $c \geqslant 0$ such that

$$
\left|e^{-\mathbf{r}\left(t_{1} H_{1}+T_{2} H_{2}\right)} E^{0} \Psi_{H_{1}}\left(m \exp \left(t_{1} H_{1}+T_{2} H_{2}\right)\right)\right| \leqslant c e^{-\varepsilon\left(t_{1}+T_{2}-T_{0}\right)}
$$

for $t_{1} \geqslant 0$ and $T_{2} \geqslant T_{0}$. Therefore by making $T_{1}, T_{2}$ tend to $+\infty$, we get

$$
\Theta_{H_{2}}(m)=\lim _{T_{1}, T_{2} \rightarrow+\infty} e^{-\mathbf{r}\left(T_{1} H_{1}+T_{2} H_{2}\right)} \Phi^{0}\left(m \exp \left(T_{1} H_{2}+T_{2} H_{2}\right)\right)
$$

Since the right side is symmetrical in $H_{1}, H_{2}$, we conclude that $\Theta_{H_{1}}(m)=\Theta_{H_{2}}(m)$.

Hence we may now write $\Theta$ instead of $\Theta_{H}$.

Lемм А 56. $\Theta(m \exp H)=e^{\Gamma(H)} \Theta(m) \quad(m \in M, H \in \mathfrak{l})$.

Since $\Omega$ is open in $\mathfrak{l}$, every $H \in \mathfrak{l}$ can be written in the form $H=\Sigma_{1 \leqslant i \leqslant q} t_{i} H_{i}\left(t_{i} \in \mathbf{R}\right.$, $H_{i} \in \Omega$ ). Our assertion therefore follows from Lemma 55.

LEMMA 57. For any $v \in \mathfrak{M}$, we can choose a number $c^{0}(v) \geqslant 0$ such that

$$
\left|e^{-t \Gamma(H)} \Phi^{0}(m \exp t H ; v)-\Theta(m ; v)\right| \leqslant c^{0}(v) \Xi_{1}(m)(1+\sigma(m))^{r_{1}} e^{-2 \varepsilon t}
$$

for $m \in M^{+}, t \geqslant 0$ and $H \in \Omega$.

$$
\text { Since } \quad \Theta(m ; v)-e^{-T \Gamma(H)} \Phi^{0}(m \exp T H ; v)=\int_{T}^{\infty} e^{-t \Gamma(H)} E^{0} \Psi_{H}(m \exp t H ; v) d t
$$

our assertion follows immediately from Lemmas 50 and 51.

CoROLLARY. For any $v \in \mathfrak{M}$, there exists a number $c(v) \geqslant 0$ such that

$$
|\Phi(m \exp t H ; v)-\Theta(m \exp t H ; v)| \leqslant c(v) \Xi_{1}(m)(1+\sigma(m))^{r_{1}} e^{-\varepsilon t}
$$

for $m \in M^{+}, H \in \Omega$ and $t \geqslant 0$.

For,

$|\Phi(m \exp t H ; v)-\Theta(m \exp t H ; v)|$

$\leqslant\left|\Phi^{+}(m \exp t H ; v)\right|+\left|\Phi^{-}(m \exp t H ; v)\right|+\left|e^{t \Gamma(H)} E^{0}\right|\left|e^{-t \Gamma(H)} \Phi^{0}(m \exp t H ; v)-\Theta(m ; v)\right|$, and so our assertion follows immediately from Lemmas 50, 52, 53 and 57.

LEMMA 58. Let $k_{1}, k_{2} \in K_{1}, m \in M$ and $\zeta \in \oiint_{1}$. Then

$$
\Theta\left(k_{1} m k_{2}\right)=\boldsymbol{\mu}_{1}\left(k_{1}\right) \Theta(m) \boldsymbol{\mu}_{2}\left(k_{2}\right), \Theta(m ; \zeta)=\boldsymbol{\Gamma}(\zeta) \Theta(m)
$$


Moreover, there exist numbers $c_{2}, r_{2} \geqslant 0$ such that

$$
|\Theta(m)| \leqslant c_{2} \Xi_{1}(m)(1+\sigma(m))^{r_{2}} \quad(m \in M) .
$$

Fix $H \in \Omega$. Since $\Gamma(H)$ commutes with the operations of $K$ on $\mathrm{V}$ (see $\S 27$ ), the first assertion follows from the relation

$$
\Theta(m)=\lim _{t \rightarrow+\infty} e^{-t \Gamma(H)} \Phi^{0}(m \exp t H)
$$

Moreover, $E^{0}, \boldsymbol{\Gamma}(H)$ and $\boldsymbol{\Gamma}(\zeta)$ commute with each other and

$$
\Phi(m \exp t H ; \zeta)=\boldsymbol{\Gamma}(\zeta) \Phi(m \exp t H)+\Psi_{\zeta}(m \exp t H)
$$

from Corollary 1 of Lemma 46. Hence

$$
\Theta(m ; \zeta)=\lim _{t \rightarrow+\infty} e^{-t \mathbf{\Gamma}(H)} \Phi^{0}(m \exp t H ; \zeta)=\Gamma(\zeta) \Theta(m)+\lim _{t \rightarrow+\infty} e^{-t \Gamma(H)} E^{0} \Psi_{\zeta}(m \exp t H)
$$

But from Lemma 50 and the corollary of Lemma 49, the limit on the right is zero. Therefor $\zeta \Theta=\boldsymbol{\Gamma}(\zeta) \Theta$.

Now put $v=1$ and $t=0$ in Lemma 57. Then we conclude from the corollary of Lemma 48 that there exists a number $c_{1} \geqslant 0$ such that

$$
|\Theta(m)| \leqslant c_{1} \Xi_{\mathbf{1}}(m)(\mathbf{l}+\sigma(m))^{r_{\mathbf{1}}} \quad\left(m \in M^{+}\right) .
$$

On the other hand, we can obviously choose a number $v_{0} \geqslant 0$ such that

$$
|\alpha(\log h)| \leqslant \nu_{0} \sigma(h) \quad\left(\alpha \in \Sigma_{2}, h \in A_{\mathfrak{p}}\right)
$$

Put $y=\max \left(1, v_{0} / 4 \varepsilon\right)$. Then it is clear (see the proof of Lemma 54) that $\mathrm{m} \exp t H \in M^{+}$ $(m \in M, H \in \Omega)$ provided $t \geqslant \nu \sigma(m)$. Now fix $m \in M$ and put $t_{0}=\nu \sigma(m)$ and $m_{0}=m \exp t_{0} H_{0}$. Then $m_{0} \in M^{+}$and

$$
\Theta(m)=e^{-t_{0} \Gamma\left(H_{\bullet}\right)} \Theta\left(m_{0}\right)
$$

Therefore

$$
|\Theta(m)| \leqslant c_{1}\left|e^{-t_{0} \Gamma\left(H_{0}\right)} E^{0}\right| \Xi_{1}\left(m_{0}\right)\left(1+\sigma\left(m_{0}\right)\right)^{r_{1}} .
$$

But $\Xi_{1}\left(m_{0}\right)=\Xi_{1}(m), \sigma\left(m_{0}\right) \leqslant \sigma(m)+t_{0}=(\nu+1) \sigma(m)$ and $\boldsymbol{\Gamma}\left(H_{0}\right) E^{0}$ has only pure imaginary eigenvalues. Therefore (see the proof of Lemma 50), the last statement of the lemma follows immediately.

For any linear function $\lambda$ on $\mathfrak{l}_{c}$, let $V(\lambda)$ denote the subspace of all $v \in V$ such that

$$
(\boldsymbol{\Gamma}(H)-\lambda(H))^{N} v=0 \quad\left(H \in \mathfrak{l}_{c}\right),
$$

where $N=\operatorname{dim} \mathbf{V}$. Let ${ }^{\circ} \mathbf{V}$ denote the sum 


$$
\sum_{\Re=0} \mathbf{V}(\lambda)
$$

where $\lambda$ runs over those linear functions which take only pure imaginary values on $\mathfrak{l}$.

LEMMA 59. $\Theta(m) \in^{0} \boldsymbol{V} \quad(m \in M)$.

Since $\Theta(m \exp t H)=e^{t \Gamma(H)} \Theta(m)(H \in \Omega, t \in \mathbf{R})$, this is obvious from the last statement of Lemma 58.

\section{\$30. Application of the induction hypothesis to $\theta$}

We recall (see $\S 7$ ) that $\mathfrak{p}$ is a Hilbert space under the norm $\|X\|(X \in \mathfrak{p})$. Put $\mathfrak{a}_{2}=\mathfrak{l}$ and let $a_{1}$ be the orthogonal complement of $\mathfrak{a}_{2}$ in $\mathfrak{a}_{\mathfrak{p}}$. It is clear that $\Sigma_{1}$ is the set of all positive roots of $\left(\mathfrak{m}, \mathfrak{a}_{\mathfrak{p}}\right)$ and $\mathfrak{l}$ is the set of those $H \in \mathfrak{a}_{\mathfrak{p}}$ where $\alpha(H)=0\left(\alpha \in \Sigma_{\mathfrak{l}}\right)$. Hence $\mathfrak{a}_{1} \subset[\mathfrak{m}, \mathfrak{m}]$. Let $\mathfrak{a}_{1}{ }^{+}$be the set of all $H \in \mathfrak{a}_{1}$ where $\alpha(H) \geqslant 0\left(\alpha \in \Sigma_{1}\right)$. Put $A_{1}{ }^{+}=\exp \mathfrak{a}_{1}{ }^{+}$and fix a number $N \geqslant 1$ such that if $h_{1} \in A_{1}{ }^{+}$and $t \geqslant N \sigma\left(h_{1}\right)$, then $h_{1} \exp t H \in A_{p^{+}}$for $H \in \Omega$. This is possible (see the proof of Lemma 58).

LEM M 60 . We can choose a number $c^{\prime} \geqslant 0$ such that

$$
\left|\Phi\left(h_{1} \exp t H\right)-\Theta\left(h_{1} \exp t H\right)\right| \leqslant c^{\prime} e^{-\varepsilon\left(t-N \sigma\left(h_{1}\right)\right)} \Xi_{1}\left(h_{1}\right)\left(1+\sigma\left(h_{1}\right)\right)^{r_{1}}
$$

for $h_{1} \in A_{1}^{+}, H \in \Omega$ and $t \geqslant N \sigma\left(h_{1}\right)$.

Put $h_{0}=h_{1} \exp t_{0} H$ where $t_{0}=N \sigma\left(h_{1}\right)$. Then $\Xi_{1}\left(h_{1}\right)=\Xi_{1}\left(h_{0}\right)$ and $\sigma\left(h_{0}\right) \leqslant \sigma\left(h_{1}\right)+N \sigma\left(h_{1}\right)\|H\|$ Since $\Omega$ is relatively compact, our assertion follows from the corollary of Lemma 57 .

Define $\theta_{j}(m) \in V$ by

and put $\theta=\theta_{1}$.

$$
\Theta(m)=\sum_{1 \leqslant j \leqslant p} \theta_{j}(m) \otimes \eta_{j}^{* *} \quad(m \in M)
$$

Corollary. Under the conditions of Lemma 60,

$$
\left|d\left(h_{1} \exp t H\right) \phi\left(h_{1} \exp t H\right)-\theta\left(h_{1} \exp t H\right)\right| \leqslant c^{\prime} e^{-\varepsilon\left(t-N \sigma\left(h_{1}\right)\right)} \Xi_{1}\left(h_{1}\right)\left(1+\sigma\left(h_{1}\right)\right)^{r_{1}} .
$$

This is obvious since $\eta_{1}=1$.

Let $\mathfrak{b}$ be the orthogonal complement of $H_{0}$ in $a_{\mathfrak{p}}$ and $\mathfrak{b}^{+}$the set of all $H \in \mathfrak{b}$ where $\alpha(H) \geqslant 0$ for every $\alpha \in \Sigma_{1}$. Let $E$ and $E_{i}$ denote the orthogonal projections of $\mathfrak{a}_{\mathfrak{p}}$ on $\mathfrak{b}$ and $\mathfrak{a}_{i}(i=1,2)$ respectively. Fix a number $\delta>0$ and let $U$ be the set of all $H \in S^{+}$such that

$$
\|E H\| \leqslant \delta\left\langle H_{0}, H\right\rangle \text {, }
$$

the scalar product being defined as in $\S 7$. We assume that $\delta$ is so small that $\delta N \leqslant \frac{1}{2}$, 
$E_{2} U \subset \Omega$ and $\alpha(H) \geqslant 3 \varepsilon$ for $\alpha \in \Sigma_{2}$ and $H \in U$. Clearly this is possible. Then $U$ consists of all elements of the form $c H_{0}+H_{1}$ where $H_{1} \in \mathfrak{b}^{+}, c^{2}+\left\|H_{1}\right\|^{2}=1$ and $\left\|H_{1}\right\| \leqslant \delta c(c>0)$, provided $\delta$ is chosen sufficiently small. Therefore, in particular, $\left(1+\delta^{2}\right)^{-\frac{1}{2}} \leqslant c \leqslant 1$. For any $T \geqslant 0$, let $U(T)$ denote the set of all elements $t H$ with $H \in U$ and $0 \leqslant t \leqslant T$.

Put $\varrho(H)=\frac{1}{2} \operatorname{tr}(\operatorname{ad} H)_{\mathfrak{n}}$ and $\varrho_{i}(H)=\frac{1}{2} \operatorname{tr}(\operatorname{ad} H)_{\mathfrak{n} i}\left(H \in \mathfrak{a}_{\mathfrak{p}}, i=1,2\right)$ in the notation of $\S 27$. Then $\varrho=\varrho_{1}+\varrho_{2}$ and we claim that $\varrho_{i}(H)=\varrho\left(E_{i} H\right)\left(H \in \mathfrak{a}_{\mathfrak{p}}\right)$. Since $\mathfrak{l}$ centralizes $\mathfrak{n}_{1}=$ $\mathfrak{m} \cap \mathfrak{n}, \varrho_{1}=0$ on $\mathfrak{a}_{2}=\mathfrak{l}$. Moreover $\mathfrak{m}$ normalizes $\mathfrak{n}_{2}$ and $\mathfrak{a}_{1} \subset[\mathfrak{m}, \mathfrak{m}]$. Therefore $\varrho_{2}=0$ on $\mathfrak{a}_{1}$ and clearly this implies our assertion. We also note that $d(\exp H)=e^{\varrho_{\mathfrak{g}}(H)}\left(H \in \mathfrak{a}_{\mathfrak{p}}\right)$.

LEMMA 61. There exists a number $c \geqslant 0$ with the following property. Suppose $\left\|H_{1}\right\| \leqslant \delta t$ $\left(H_{1} \in \mathfrak{b}^{+}, t \geqslant 0\right)$. Then

$$
\left|e^{\varrho\left(t H_{0}+E_{3} H_{1}\right)} \phi\left(\exp \left(t H_{0}+H_{1}\right)\right)-\theta\left(\exp \left(t H_{0}+H_{1}\right)\right)\right| \leqslant c e^{-\varepsilon t / 3} e^{-\varrho_{1}\left(H_{1}\right)} .
$$

If $\alpha \in \Sigma_{1}$, it is clear that $\alpha\left(E_{1} H_{1}\right)=\alpha\left(H_{1}\right) \geqslant 0$. Hence $E_{1} H_{1} \in \mathfrak{a}_{1}{ }^{+}$. Moreover $N\left\|E_{1} H_{1}\right\| \leqslant$ $N\left\|H_{1}\right\| \leqslant N \delta t \leqslant t / 2$. Therefore it follows without difficulty from the corollary of Lemma 60 that the left side is majorized by

$$
c^{\prime} e^{-s t / 2} \Xi_{1}\left(h_{1}\right)\left(1+\sigma\left(h_{1}\right)\right)^{r_{1}},
$$

where $h_{1}=\exp E_{1} H_{1}$. Therefore our assertion follows by applying [4 (j), Theorem 3] to $\Xi_{1}$ and observing that $\sigma\left(h_{1}\right)=\left\|E_{1} H_{1}\right\| \leqslant \delta t$.

As in $\S 7$, put

$$
D(h)=\prod_{\alpha \in \Sigma}\left\{e^{\alpha(\log h)}-e^{-\alpha(\log h)}\right\}^{m_{\alpha}} \quad\left(h \in A_{\mathfrak{p}}\right)
$$

where $m_{\alpha}$ is the multiplicity of $\alpha$. Then $D(h) \geqslant 0$ for $h \in A_{\mathfrak{p}}^{+}$. Put

$$
F_{U}(T)=\int_{\log h \in U(T)}|\phi(h)|^{2} D(h) d h \quad(T \geqslant 0)
$$

where $d h$ is the Haar measure on $A$. Also let us recall that $\phi \neq 0$ and it is analytic (see [4 (q), Lemma 33]).

Lемма 62. There exists a unique integer $v \geqslant 0$ with the following property. We can select numbers $a, b(0<a \leqslant b<\infty)$ such that

$$
a T^{v} \leqslant F_{U}(T) \leqslant b T^{v}
$$

for all $T \geqslant 1 . \nu=0$ if and only if $\theta=0$.

The uniqueness of $v$ is obvious from its definition. So we have only to verify its existence. 
Let $U_{0}(T)$ denote the subset of all $H \in \mathfrak{a}_{\mathfrak{p}}$ of the form $H=t H_{0}+H_{1}$ where $0 \leqslant t \leqslant T$, $H_{1} \in \mathfrak{b}^{+}$and $\left\|H_{1}\right\| \leqslant \delta t$. Then

$$
U(T) \subset U_{0}(T) \subset U\left(T\left(1+\delta^{2}\right)^{\frac{1}{2}}\right)
$$

Put

$$
F_{0}(T)=\int_{\log h \in U_{0}(T)}|\phi(h)|^{2} D(h) d h
$$

Then

$$
F_{0}\left(T\left(1+\delta^{2}\right)^{-\frac{1}{2}}\right) \leqslant F(T) \leqslant F_{0}(T),
$$

where $F=F_{U}$. Therefore it would be enough to prove the existence of an integer $v \geqslant 0$ such that

$$
0<\liminf _{T \rightarrow \infty} T^{-v} F_{0}(T) \leqslant \limsup _{T \rightarrow \infty} T^{-v} F_{0}(T)<\infty
$$

For any $c \geqslant 0$, let $\mathfrak{b}^{+}(c)$ denote the set of all $H \in \mathfrak{b}^{+}$with $\|H\| \leqslant c$. Then if $\psi(H)=$ $\phi(\exp H)\left(H \in \mathfrak{a}_{\mathfrak{p}}\right)$, we have

$$
F_{0}(T)=\int_{0}^{T} d t \int_{\mathfrak{b}^{+}(\delta t)}\left|e^{\varrho\left(t H_{\mathbf{0}}+E_{2} H_{1}\right)} \psi\left(t H_{0}+H_{1}\right)\right|^{2} D_{1}\left(H_{1}\right) \prod_{\alpha \in \Sigma_{2}}\left(1-e^{-2 \alpha\left(t H_{0}+H_{1}\right)}\right)^{m_{\alpha}} d H_{1},
$$

where

$$
D_{1}\left(H_{1}\right)=\prod_{\alpha \in \Sigma_{1}}\left(e^{\alpha\left(H_{1}\right)}-e^{-\alpha\left(H_{1}\right)}\right)^{m_{\llcorner}} \quad\left(H_{1} \in \mathfrak{b}\right)
$$

and $d H_{1}$ is the (suitably normalized) Euclidean measure on $\mathfrak{b}$. Choose $T_{0} \geqslant 0$ so large that $2 \alpha\left(t H_{0}+H_{1}\right) \geqslant \log 2$ for $\alpha \in \Sigma_{2}, H_{1} \in \mathfrak{b}^{+}(\delta t)$ and $t \geqslant T_{0}$, and put

$$
J(T)=\int_{T_{0}}^{T} d t \int_{\mathfrak{b}^{+}(\delta t)}\left|e^{\varrho\left(t H_{0}+E_{\mathbf{q}} H_{1}\right)} \psi\left(t H_{0}+H_{1}\right)\right|^{2} D_{1}\left(H_{1}\right) d H_{1}
$$

for $T \geqslant T_{0}$. Then if $q=\operatorname{dim} \mathfrak{n}_{2}$, it is clear that

$$
2^{-q} J(T) \leqslant F_{0}(T)-F_{0}\left(T_{0}\right) \leqslant J(T) \quad\left(T \geqslant T_{0}\right)
$$

Also put $\psi_{\infty}(H)=\theta(\exp H)\left(H \in \mathfrak{a}_{\mathfrak{p}}\right)$ and

$$
J_{\infty}(T)=\int_{T_{0}}^{T} d t \int_{\mathfrak{b}^{+}(\delta t)}\left|\psi_{\infty}\left(t H_{0}+H_{1}\right)\right|^{2} D_{1}\left(H_{1}\right) d H_{1}
$$

Then, by the triangle inequality, we have

$$
\left|J(T)^{\frac{1}{2}}-J_{\infty}(T)^{\frac{1}{2}}\right|^{2} \leqslant \int_{T_{0}}^{T} d t \int_{\mathfrak{b}^{+}(\delta t)}\left|e^{\varrho\left(t H_{0}+E_{2} H_{1}\right)} \psi\left(t H_{0}+H_{1}\right)-\psi_{\infty}\left(t H_{0}+H_{1}\right)\right|^{2} D_{1}\left(H_{1}\right) d H_{1} .
$$

Now apply Lemma 61 and observe that 


$$
e^{-2 \varrho_{1}\left(H_{1}\right)} D_{1}\left(H_{1}\right) \leqslant 1 \quad\left(H_{1} \in \mathfrak{b}^{+}\right) .
$$

Then it follows easily that

$$
\left|J(T)^{\frac{1}{2}}-J_{\infty}(T)^{\frac{1}{2}}\right| \leqslant c \quad\left(T \geqslant T_{0}\right),
$$

where $c$ is a fixed positive number.

Now first suppose $\theta=0$. Then $\psi_{\infty}=0$ and therefore $J_{\infty}(T)=0$. Hence $J\left(T() \leqslant c^{2}\right.$ for all $T \geqslant T_{0}$ and it is clear that we can take $\nu=0$.

So let us now suppose that $\theta \neq 0$. Then it is obvious from Lemma 58 that all the assumptions of Lemma 42 are fulfilled if we replace $(G, K, \phi)$ by $\left(M, K_{1}, \theta\right)$. Since $\operatorname{dim} \mathfrak{m}<\operatorname{dim} \mathfrak{g}$

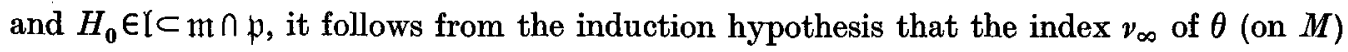
is positive. Hence it follows from Lemma 44 and [4 (d), Lemma 38] that

$$
0<\liminf _{T \rightarrow \infty} T^{-v_{\infty}} J_{\infty}(T) \leqslant \limsup _{T \rightarrow \infty} T^{-y} \infty J_{\infty}(T)<\infty
$$

Since $\nu_{\infty} \geqslant 1$, it is clear that similar inequalities hold if we replace $J_{\infty}(T)$ by $J(T)$. Hence we can take $\nu=\nu_{\infty}$.

LqmMA 63. Define $v$ as in Lemma 62. Then for any $\varepsilon_{1}>0$, we can choose $T_{0}>0$ and $\delta_{1}>0$ such that

for $T_{0} \leqslant T_{1} \leqslant T_{2} \leqslant\left(1+\delta_{1}\right) T_{1}$

$$
T_{1}^{-v}\left\{F_{U}\left(T_{2}\right)-F_{U}\left(T_{1}\right)\right\} \leqslant \varepsilon_{1}
$$

Put $F=F_{U}$ and let $0 \leqslant T_{1} \leqslant T_{2}$. Then

$$
F\left(T_{2}\right)-F\left(T_{1}\right) \leqslant \int_{\mathfrak{\alpha}\left(T_{1}, T_{2}\right)}\left|e^{\varrho\left(t H_{0}+E_{2} H_{1}\right)} \psi\left(t H_{0}+H_{1}\right)\right|^{2} D_{1}\left(H_{1}\right) d t d H_{1},
$$

where $\mathfrak{a}\left(T_{1}, T_{2}\right)$ is the set of all points in $\mathfrak{a}_{\mathfrak{p}}$ of the form $t H_{0}+H_{1}$ with $t \geqslant 0, H_{1} \in \mathfrak{b}^{+}(\delta t)$ and $T_{1}^{2} \leqslant t^{2}+\left\|H_{1}\right\|^{2} \leqslant T_{2}^{2}$. Therefore

$$
\left\{F\left(T_{2}\right)-F\left(T_{1}\right)\right\}^{\frac{1}{2}} \leqslant I_{1}\left(T_{1}, T_{2}\right)^{\frac{1}{2}}+I_{2}\left(T_{1}, T_{2}\right)^{\frac{1}{3}},
$$

where $\quad I_{1}\left(T_{1}, T_{2}\right)=\int_{\mathfrak{a}\left(T_{1}, T_{2}\right)}\left|\psi_{\infty}\left(t H_{0}+H_{1}\right)\right|^{2} D_{1}\left(H_{1}\right) d t d H_{1}$

and

$$
I_{2}\left(T_{1}, T_{2}\right)=\int_{\mathfrak{a}\left(T_{1}, T_{2}\right)}\left|e^{\varrho\left(t H_{\mathfrak{0}}+E_{2} H_{1}\right)} \psi\left(t H_{0}+H_{1}\right)-\psi_{\infty}\left(t H_{0}+H_{1}\right)\right|^{2} D_{1}\left(H_{1}\right) d t d H_{1}
$$

If we apply Lemma 61 and observe, as before, that $e^{-2 \varrho_{1}\left(H_{1}\right)} D_{1}\left(H_{1}\right) \leqslant 1$ for $H_{1} \in \mathfrak{b}^{+}$, it follows easily that 


$$
I_{2}\left(T_{1}, T_{2}\right) \leqslant c_{2} e^{-\varepsilon^{\prime} T_{1}} \quad\left(T_{2} \geqslant T_{1} \geqslant 0,\right.
$$

where $c_{2}$ is a fixed positive number and $\varepsilon^{\prime}=\left(1+\delta^{2}\right)^{-\frac{1}{2}} \cdot \varepsilon / 2$.

Now first suppose $\theta=0$. Then $\nu=0$ and $I_{1}\left(T_{1}, T_{2}\right)=0$. Hence

$$
F\left(T_{2}\right)-F\left(T_{1}\right) \leqslant I_{2}\left(T_{1}, T_{2}\right) \leqslant c_{2} e^{-\epsilon^{\prime} T_{1}}
$$

and the statement of Lemma 63 follows immediately. So let us assume that $\theta \neq 0$. Then $\nu=v_{\infty} \geqslant 1$, as we have seen above, and therefore the required assertion is a consequence of Lemma 45.

\section{§ 31. Completion of the proofs of Lemmas 42 and 43}

We shall now finish the proofs of Lemmas 42 and 43 . For any open subset $U$ of $S^{+}$ define

$$
F_{U}(T)=\int_{\log h \in U(T)}|\phi(h)|^{2} D(h) d h \quad(T \geqslant 0)
$$

where $U(T)$ is the set of all elements in $\mathfrak{a}_{\mathfrak{p}}{ }^{+}$of the form $t H(0 \leqslant t \leqslant T, H \in U)$. Since $S^{+}$is compact, we can choose open sets $U_{i}$ in $S^{+}$and integers $\nu_{i} \geqslant 0(1 \leqslant i \leqslant q)$ such that $S^{+}=$ $U_{1 \leqslant i \leqslant q} U_{i}$ and the statements of Lemmas 62 and 63 hold for $\left(U_{i}, v_{i}\right)$ in place of $(U, v)$. Put $F_{i}=F_{U_{i}}$ and $\nu=\max _{i} v_{i}$. It is clear (see [4 (d), Lemma 38] that

$$
\max _{i} F_{i}(T) \leqslant\|\phi\|_{T}^{2} \leqslant \sum_{1 \leqslant i \leqslant q} F_{i}(T)
$$

if the measure $d h$ is suitably normalized. So it is obvious that

$$
0<\liminf _{T \rightarrow \infty} T^{-v / 2}\|\phi\|_{T} \leqslant \limsup _{T \rightarrow \infty} T^{-v / 2}\|\phi\|_{T}<\infty .
$$

Moreover, $\quad\|\phi\|_{T_{2}}{ }^{2}-\|\phi\|_{T_{1}}{ }^{2} \leqslant \sum_{1 \leqslant i \leqslant q}\left\{F_{i}\left(T_{2}\right)-F_{i}\left(T_{1}\right)\right\} \quad\left(0 \leqslant T_{1} \leqslant T_{2}\right)$.

Therefore the last assertion of Lemma 42 follows immediately from Lemma 63 .

Now we come to the proof of Lemma 43. First assume that $\nu=0$ and fix $H_{0} \in S^{+}$. Then $v_{i}=0(1 \leqslant i \leqslant q)$ in the above proof. We may suppose that $H_{0} \in U_{1}$. Define $U$ as in $\S 30$ for $H_{0}$ and let $A_{\mathfrak{p}}(U)$ denote the set of all $h \in A_{\mathfrak{p}}$ of the form $h=\exp t H(t \geqslant 0, H \in U)$. We may obviously assume that $U \subset U_{1}$. Then it follows from Lemma 62 that $\theta=0$ and therefore

$$
\left|e^{o(t H)} \phi(\exp t H)\right| \leqslant c e^{-\varepsilon^{\prime} t} \quad(H \in U, t \geqslant 0)
$$

from Lemma 61 where $\varepsilon^{\prime}=\left(1+\delta^{2}\right)^{-\frac{t}{2}} \cdot \varepsilon / 3$. Hence it is clear (see [4 (j), Lemma 36]) that 


$$
\sup _{h \in A_{\mathfrak{p}}(U)}|\phi(h)| \Xi(h)^{-1}(1+\sigma(h))^{r}<\infty
$$

for every $r \geqslant 0$. Since $G=K A_{\mathfrak{p}}+K$ and $S^{+}$is compact, this means that

$$
\sup _{x \in G}|\phi(x)| \Xi(x)^{-1}(1+\sigma(x))^{r}<\infty
$$

for any $r \geqslant 0$. But then it follows easily (see the proof of Lemma 48) that $\phi \in C(G) \otimes V$. Thus 1) implies 3) in Lemma 43. In view of Lemma 11 and [4 (j), Theorem 3], it is obvious that 3) implies both 1) and 2). Hence it remains to prove that 2) implies 1).

So suppose 2) holds. Fix $H_{0} \in S^{+}$and use the notation of $\S 30$. Then it follows from Lemma 61 that

$$
\lim _{t \rightarrow+\infty} \theta\left(\exp \left(t H_{0}+H_{1}\right)\right)=0 \quad\left(H_{1} \in \mathfrak{b}^{+}\right)
$$

Now fix $H_{1} \in \mathfrak{b}^{+}$and put $\left.f(t)=\theta\left(\exp \left(t H_{0}+H_{1}\right)\right)(t \in \mathbf{R}).\right)$. We have seen in $\S 29$ that

$$
\Theta\left(\exp \left(t H_{0}+H_{1}\right)\right)=e^{t \Gamma\left(H_{0}\right)} \Theta\left(\exp H_{1}\right)
$$

and all eigenvalues of $\Gamma\left(H_{0}\right) E^{0}$ are pure imaginary. Hence it is clear that

$$
f(t)=\sum_{1 \leqslant i \leqslant r} p_{i}(t) e^{(-1)^{\frac{1}{2} \lambda_{i} t}} \quad(t \in \mathbf{R}),
$$

where $\lambda_{1}, \ldots, \lambda_{r}$ are distinct real numbers and $p_{i}$ are polynomial functions from $\mathbf{C}$ to $V$. Since $f(t) \rightarrow 0$ as $t \rightarrow+\infty$, we conclude (see $[4(\mathrm{j}), \S 15]$ ) that $f=0$. Since $\mathfrak{b}^{+}+\mathbf{R} H_{0}$ is open in $a_{p}$ and $\theta$ is analytic (see [4(q), Lemma 33]), it follows that $\theta=0$ and therefore $v=0$ in Lemma 62. This being true for every $H_{0} \in S^{+}$, we conclude (see the proof of Lemma 42 given above) that the index of $\phi$ is zero. This shows that 2) implies 1) and so the proof of Lemma 43 is now complete.

\section{Part III. Applications to harmonic analysis on $G$}

\section{\$ 32. Lemma 64 and its consequences}

Let $\mathfrak{a}=\theta(\mathfrak{a})$ be a Cartan subalgebra of $\mathfrak{g}$ and $A$ the corresponding Cartan subgroup of $G$. For $f \in \mathcal{C}(G)$, define $F_{f} \in \mathcal{C}\left(A^{\prime}(I)\right)$ as in $\S 18$. Let $J$ be the algebra of all invariants of $W(\mathfrak{g} / \mathfrak{a})$ in $\mathfrak{A}=\mathfrak{S}\left(\mathfrak{a}_{\mathfrak{c}}\right)$. Then we have a canonical isomorphism $\gamma$ of 8 onto $\mathbf{J}$ (see [4 (e), Lemma 19]). Moreover, since $C_{c}{ }^{\infty}(G)$ is dense in $\mathrm{C}(G)$, it follows (see $\S 18$ and [4 (o), $\left.\S 22\right]$ ) that

$$
F_{z f}=\gamma(z) F_{f} \quad(z \in \Re, f \in \mathrm{C}(G))
$$




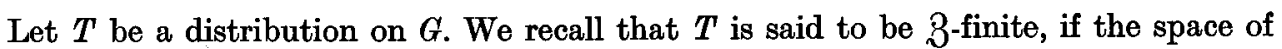
distributions of the form $z T(z \in \beta)$ has finite dimension. In particular, we can speak of a locally summable function being 8 -finite.

Lемма 64. Let $f$ be a 8-finite function in $\mathcal{C}(G)$. Then $F_{f}=0$ unless $\mathfrak{a} \cap \mathfrak{p}=\{0\}$.

Put $\mathfrak{a}_{1}=\mathfrak{a} \cap \mathfrak{f}, \mathfrak{a}_{2}=\mathfrak{a} \cap \mathfrak{p}, A_{1}=A \cap K$ and $A_{2}=\exp \mathfrak{a}_{2}$ and suppose that $\mathfrak{a}_{2} \neq\{0\}$. Fix a point $a \in A^{\prime}(I)$ and let $a=a_{1} a_{2}\left(a_{i} \in A_{i}, i=1,2\right)$. Select an open and connected neighborhood $\mathfrak{a}_{1}^{0}$ of zero in $\mathfrak{a}_{1}$ such that

$$
a_{1} \exp \mathfrak{a}_{1}{ }^{0} \cdot A_{2} \subset A^{\prime}(I) .
$$

This is clearly possible. Put $\mathfrak{a}^{0}=\mathfrak{a}_{1}{ }^{0}+\mathfrak{a}_{2}$ and $A^{0}=a_{1} \exp \mathfrak{a}^{0}$.

Let $\mathfrak{U}$ be the set of all $u \in B$ such that $u f=0$. Then $\mathfrak{U}$ is an ideal in 8 of finite codimension. Since $\mathfrak{A}$ is a finite module over $J$, it follows that $\mathfrak{B}=\mathfrak{A} \gamma(\mathfrak{U})$ has finite codimension in $\mathfrak{A}$. Moreover $\gamma(u) F_{f}=F_{u f}=0(u \in \mathfrak{U})$ and therefore $v F_{f}=0$ for $v \in \mathfrak{B}$. Since $\mathfrak{a}^{0}$ is connected, we conclude from $[4(\mathrm{e}), \mathrm{p} .131]$ that

$$
F_{f}\left(a_{1} \exp H\right)=\sum_{1 \leqslant i \leqslant r} p_{i}(H) e^{\lambda_{\mathbf{i}}(H)} \quad\left(H \in \mathfrak{a}^{0}\right),
$$

where $\lambda_{i}$ are linear functions and $p_{i}$ plynomial functions on $\mathfrak{a}_{c}(1 \leqslant i \leqslant r)$. Put

$$
g(H)=F_{f}\left(a_{1} \exp H\right) \quad\left(H \in \mathfrak{a}_{2}\right)
$$

Since $F_{f} \in \mathrm{C}\left(A^{\prime}(I)\right)$, it is clear that

$$
\sup _{\mathfrak{a}_{\mathbf{a}}}|g(H)|(1+\|H\|)^{m}<\infty
$$

for any $m \geqslant 0$. Since $\mathfrak{a}_{2} \neq\{0\}$, we conclude from $[4(\mathrm{j}), \S 15]$ that $g=0$. This shows that $F_{f}=0$ on $a_{1} A_{2}$ and therefore $F_{f}(a)=0$.

Corollary 1. Suppose $f \neq 0$ in the above lemma. Then $\operatorname{rank} G=\operatorname{rank} K$.

For let us otherwise assume that rank $G>\operatorname{rank} K$. Choose a so that it is fundamental in $g$. Then $a \cap \mathfrak{p} \neq\{0\}$ and therefore $F_{f}=0$. But then it follows from Lemma 38 that $f(1)=0$. Now fix $x \in G$ and put $f_{x}=r(x) f$ in the notation of $\S 10$. Then $f_{x}$ is also a 8 -finite function in $C(G)$ and $f(x)=f_{x}(1)=0$, from the above proof. This shows that $f=0$, giving a contradiction.

COROLLARY 2. Suppose $\operatorname{rank} G=\operatorname{rank} K$ and $f$ is a 3-finite function in $\mathrm{C}(G)$. Then in the notation of Theorem 8.

$$
c f(1)=(-1)^{q} \sum_{\lambda \in L} \varpi(\lambda) \Theta_{\lambda}(f)
$$


It is clear from Lemma 64 that $\phi_{f, i}=0(2 \leqslant i \leqslant r)$ in Theorem 8. Hence our assertion is obvious.

An element $\gamma$ of $G$ is called elliptic if it is contained in some compact Cartan subgroup.

COROLLARY 3. Suppose $\gamma$ is a semisimple element of $G$ which is not elliptic. Then if $f$ is a B-finite function in $\mathrm{C}(G)$, we can conclude that

in the notation of Lemma 28.

$$
\int_{G / G_{\gamma}} f\left(\gamma^{\bar{x}}\right) d \dot{x}=0
$$

Define $z$ and $\mathfrak{h}$ as in Theorem 6 . By replacing $\gamma$ by $\gamma^{y}$ for some $y \in G$, we may assume that $\theta(\mathfrak{h})=\mathfrak{h}$. Let $A$ be the Cartan subgroup of $G$ corresponding to $\mathfrak{h}$. Then $\gamma \in A$. Since $\gamma$

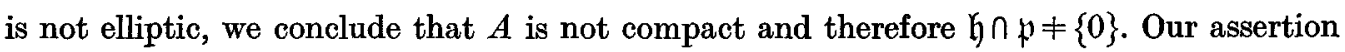
now follows from Lemmas 28 and 64 .

\section{§ 33. Proof of a conjecture of Selberg}

Let $f$ be a measurable function on $G$. We say that $\left({ }^{1}\right) f$ is $K$-finite, if the left and right translates of $f$, under $K$, span a finite-dimensional space.

LEмM А 65. Let $f$ be a function in $C^{\infty}(G) \cap L_{2}(G)$ which is K-finite as well as 8-finite. Then there exist numbers $c, r \geqslant 0$ such that

$$
|f(x)| \leqslant c \Xi(x)(1+\sigma(x))^{r} \quad(x \in G) .
$$

We shall give a proof of this lemma in $\S 38$.

COROLLARY 1. Let $f$ be a function in $L_{2}(G)$ which is both $K$-finite and 8-finite. Then $f \in C(G)$.

We regard $\mathfrak{H}=L_{2}(K \times K)$ as a Hilbert space in the usual way and define a unitary double representation $\mu^{0}=\left(\mu_{1}, \mu_{2}\right)$ of $K$ on $\mathfrak{g}$ as follows (cf. $\S 12$ ). If $u \in \mathfrak{g}$ and $k \in K$, then the functions $u_{1}=\mu_{1}(k) u$ and $u_{2}=u \mu_{2}(k)$ are given by

$$
u_{1}\left(k_{1}, k_{2}\right)=u_{1}\left(k^{-1} k, k_{2}\right), u_{2}\left(k_{1}, k_{2}\right)=u\left(k_{1}, k_{2} k^{-1}\right) \quad\left(k_{1}, k_{2} \in K\right) .
$$

It follows from [4 (q), Lemma 33] that $f$ is analytic. For any $x \in G$, let $\phi(x)$ denote the function

(1) We do not distinguish between two measurable functions which differ only on a set of measure zero. 


$$
\left(k_{1}, k_{2}\right) \rightarrow f\left(k_{1}^{-1} x k_{2}^{-1}\right) \quad\left(k_{1}, k_{2} \in K\right)
$$

in $\mathfrak{G}$. Then it is clear that $\phi\left(k_{1} x k_{2}\right)=\mu_{1}\left(k_{1}\right) \phi(x) \mu_{2}\left(k_{2}\right)\left(k_{1}, k_{2} \in K\right)$. Let $V$ be the subspace of $\mathfrak{H}$ spanned by $\phi(x)$ for all $x \in G$. Then $V$ is stable under $\mu^{0}$ and $\operatorname{dim} V<\infty$ since $f$ is $K$-finite. Let $\mu$ denote the restriction of $\mu^{0}$ on $V$. Then $\phi$ is a $C^{\infty} \mu$-spherical function from $G$ to $V$ and it is clear from Lemma 65 that Lemmas 42 and 43 are applicable to $\phi$, provided $f \neq 0$. Since $f \in L_{2}(G)$, we conclude that the index of $\phi$ is zero and therefore $\phi \in C(G) \otimes V$ from Lemma 43. Obviously this implies that $f \in C(G)$.

Coroldary 2. Suppose $f \neq 0$ in Corollary 1. Then $\operatorname{rank} G=\operatorname{rank} K$.

This follows immediately from Corollary 1 of Lemma 64.

If we combine Corollary 3 of Lemma 64 with Corollary 1 of Lemma 65, we get the following theorem.

THEOREM 11. Suppose $\gamma$ is a semisimple element of $G$, which is not elliptic, and $f$ a function in $L_{2}(G)$, which is both $K$-finite and 3-finite. Then $f \in \mathcal{C}(G)$ and, in the notation of Lemma 28, the integral

exists and its value is zero.

$$
\int_{G / G_{\gamma}} f\left(\gamma^{\bar{x}}\right) d \bar{x}
$$

This theorem represents, essentially, a conjecture of Selberg $[9, \mathrm{p} .70]$. I understand that R. P. Langlands had obtained a similar but somewhat weaker result, a few years ago.

\section{§ 34. The behaviour of certain eigenfunctions at infinity}

We now return to the notation of $\S 27$. Extend $a_{\mathfrak{p}}$ to a Cartan subalgebra $\mathfrak{a}$ of $\mathfrak{g}$. Define $\varpi=\varpi^{\mathfrak{a}}, W=W(\mathfrak{g} / \mathfrak{a})$ and $W_{1}=W(\mathfrak{m} / \mathfrak{a})$ as usual (see $[4(\mathrm{p}), \S 12]$ ) and, for a given linear function $\lambda$ on $\mathfrak{a}_{e}$, put

$$
\chi_{\lambda}(z)=\chi_{\lambda}{ }^{a}\left(p_{z}\right) \quad(z \in 8)
$$

in the notation of $[4(p), \S 12]$ and $[4(0), \S 14]$. Let $\mathfrak{U}_{\lambda}$ denote the kernel of $\chi_{\lambda}$ in 8 and put $\mathfrak{U}_{1 \lambda}=8_{1} \mu_{0}\left(\mathfrak{U}_{\lambda}\right), B_{1 \lambda}{ }^{*}=8_{1} / \mathfrak{U}_{1 \lambda}$. (Here $\mu_{0}=\mu_{\mathrm{g} / \mathrm{m}}$ as in $\S 27$.) Let $\sigma_{\lambda}$ denote the natural representation of $B_{1}$ on $81 \lambda^{*}$.

Let $r=\left[W: W_{1}\right]$ and select elements $s_{1}=1, s_{2}, \ldots, s_{\tau}$ in $W$ such that $W=\mathrm{U}_{1 \leqslant i \leqslant r} W_{1} s_{i}$. Consider the subalgebras $J$ and $J_{1}$ of all invariants of $W$ and $W_{1}$ respectively in $\subseteq\left(a_{c}\right)$. Then we have the canonical isomorphisms $\gamma: B \rightarrow J$ and $\gamma_{1}: \bigcap_{1} \rightarrow J_{1}$ (see [4(o), § 12]) and

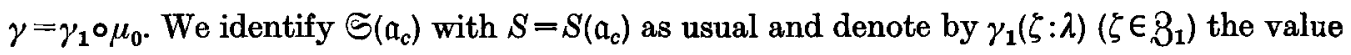
at $\lambda$ of the element $\gamma_{1}(\zeta) \in S$. 
LeMma 66. $\operatorname{dim} 8_{1 \lambda}{ }^{*}=r$ and, if $\varpi(\lambda) \neq 0$, we can choose a base $\left(v_{1}, \ldots, v_{r}\right)$ for $8_{1} \lambda^{*}$ such that

$$
\sigma_{\lambda}(\zeta) v_{i}=\gamma_{1}\left(\zeta: s_{i} \lambda\right) v_{i} \quad\left(\zeta \in B_{1}, 1 \leqslant i \leqslant r\right) .
$$

Since $W$ and $W_{1}$ are both generated by reflexions, the results of $[4(\mathrm{j}), \S 3]$ are applicable. Therefore by taking into account the isomorphisms $\gamma$ and $\gamma_{1}$, our assertions follow immediately from Lemmas 13 and 15 of [4 (j)].

Let $\mu$ and $V$ have the same meaning as in Lemma 42.

LEM M A 67. Let $\lambda$ be a linear function on $\mathfrak{a}_{c}$ and $\phi$ a $C^{\infty} \mu$-spherical function from $G$ to $V$. Suppose the following conditions are fulfilled:

1) $\operatorname{rank} G=\operatorname{rank} K$.

2) $\lambda$ takes only real values on $\mathfrak{a} \cap \mathfrak{p}+(-1)^{\mathfrak{t}} \mathfrak{a} \cap \mathfrak{f}$ and $\varpi(\lambda) \neq 0$.

3) $z \phi=\chi_{\lambda}(z) \phi \quad(z \in 3)$.

4) There exist numbers $c, s \geqslant 0$ such that

Then $\phi \in \mathcal{C}(G) \otimes V$.

$$
|\phi(x)| \leqslant c \Xi(x)(1+\sigma(x))^{s} \quad(x \in G) .
$$

We may obviously assume that $\phi \neq 0$ and $G$ is not compact so that $\mathfrak{a}_{\mathfrak{p}} \neq\{0\}$. Then, in view of Lemma 43 , it would be enough to verify that

$$
\lim _{t \rightarrow+\infty} e^{t e(H)} \phi(h \exp t H)=0
$$

for $H \in S^{+}$and $h \in A_{\mathfrak{p}^{+}}$. For any $H_{0} \in S^{+}$, let $\mathfrak{m}_{H_{0}}$ denote the centralizer of $H_{0}$ in $\mathrm{g}$. Suppose the above condition does not hold. Then we can choose $H_{0} \in S^{+}$such that:

1) For some $h \in A_{\mathfrak{p}^{+}}, e^{t_{\ell\left(H 0_{0}\right)}} \phi\left(h \exp t H_{0}\right)$ does not tend to zero as $t \rightarrow+\infty$.

2) $\operatorname{dim} \mathfrak{m}_{H_{0}}$ is minimum possible consistent with condition 1 ).

Put $\mathfrak{m}=\mathfrak{m}_{H_{0}}, \mathfrak{m}_{1}=\mathfrak{m} \cap \mathfrak{f}+[\mathfrak{m}, \mathfrak{m}] \cap \mathfrak{p}$ and let $\mathfrak{l}$ be the centralizer of $\mathfrak{m}$ in $\mathfrak{p}$. Then $\mathfrak{n t}_{1} \cap \mathfrak{p}$ is the orthogonal complement of $\mathfrak{l}$ in $\mathfrak{m} \cap \mathfrak{p}$. Let $M_{1}$ be the analytic subgroup of $G$ corresponding to $m_{1}$. We now use the notation of $\$ \S 27-30$ for this particular $H_{0}$. (Note that $\mathfrak{c}_{\mathfrak{p}}=\{0\}$ in the present case since rank $\mathfrak{g}=\operatorname{rank}$.) Define $\Theta(m)$ and $\theta(m)(m \in M)$ as in $\S \S 29,30$. We know from Lemma 66 that the representation $\Gamma$ of $\bigotimes_{1}$ is semisimple. Moreover it is clear from condition 2) of Lemma 67 that $s_{i} \lambda$ takes only real values on $\mathfrak{a}_{\mathfrak{p}}$ and therefore also on $\mathfrak{l}$. Hence we conclude from Lemma 59 that

$$
\Theta(m \exp H)=\Theta(m) \quad(m \in M, H \in \mathfrak{l}) .
$$

This implies, in particular, that 


$$
\theta(m \exp H)=\theta(m) \quad(m \in M, H \in \mathfrak{l})
$$

Hence it follows from Lemma 61 and the definition of $H_{0}$, that $\theta \neq 0$.

Fix an element $H \neq 0$ in $\mathfrak{a}_{1}{ }^{+}$. Then if $h \in A_{1}{ }^{+}$, we claim that

$$
e^{t \operatorname{o}_{\mathbf{1}}(H)} \theta(h \exp t H) \rightarrow 0
$$

as $t \rightarrow+\infty$. Put $H_{1}=c_{1}\left(H_{0}+c H\right)$, where $c$ is a small positive number and $c_{1}=\left\|H_{0}+c H\right\|^{-1}$. Then $H_{1} \in S^{+}$and it is obvious that

$$
\operatorname{dim} \mathfrak{m}_{H_{1}}<\operatorname{dim} \mathfrak{m}_{H_{0}} \text {. }
$$

Hence we conclude from definition of $H_{0}$ that

$$
e^{t \mathfrak{t e}\left(H_{1}\right)} \phi\left(h_{0} \exp t H_{1}\right) \rightarrow 0 \quad\left(h_{0} \in A_{\mathfrak{p}}{ }^{+}\right)
$$

as $t \rightarrow+\infty$. Define $U$ as in $\S 30$ and let $U_{0}$ denote the interior of $U$ in $S^{+}$. Then, by choosing $c$ sufficiently small, we can assume that $H_{1} \in U_{0}$ and therefore

$$
\lim _{t \rightarrow+\infty}\left|e^{t Q\left(H_{1}\right)} \phi\left(h \exp t H_{1}\right)-e^{\varrho_{1}\left(t H_{1}\right)} \theta\left(h \exp t H_{1}\right)\right|=0 \quad\left(h \in A_{1}^{+}\right)
$$

from Lemma 61. Fix $h \in A_{1}{ }^{+}$. Since $\alpha\left(H_{1}\right)>0 \quad\left(\alpha \in \Sigma_{2}\right)$, we can choose $t_{0} \geqslant 0$ such that $h_{0}=h \exp t_{0} H_{1} \in A_{\mathfrak{p}}+$. Hence it follows from what we have seen above that

$$
e^{t e\left(H_{1}\right)} \phi\left(h \exp t H_{1}\right) \rightarrow 0
$$

as $t \rightarrow+\infty$. Put $c_{2}=c_{1} c$. Then $\varrho_{1}\left(H_{1}\right)=c_{2} \varrho_{1}(H)$ and $H_{1}=c_{1} H_{0}+c_{2} H$. Therefore since $H_{0} \in \mathfrak{l}$, we conclude that

and this proves our assertion.

$$
e^{t \varrho_{1}(H)} \theta(h \exp t H) \rightarrow 0
$$

Let $\theta_{1}$ denote the restriction of $\theta$ on $M_{1}$. It is clear that $\theta_{1} \div 0$ and we conclude from Lemmas 43 and 58 that $\theta_{1} \in \mathcal{C}\left(M_{1}\right) \otimes V$. But then rank $\mathfrak{m}_{1}=\operatorname{rank}\left(\mathfrak{m}_{1} \cap \mathfrak{l}\right)$ from Corollary 1 of Lemma 64.

Fix a Cartan subalgebra $\mathfrak{c}$ of $\mathfrak{m}_{\mathfrak{1}} \cap \mathfrak{f}$. Then $\mathfrak{h}=\mathfrak{l}+\mathfrak{c}$ is a Cartan subalgebra of $\mathfrak{m}$ and therefore also of $\mathfrak{g}$. Since rank $\mathfrak{g}=\operatorname{rank} \mathfrak{l}$ and $H_{0} \in \mathfrak{l}, \mathfrak{h}$ cannot be fundamental in $\mathfrak{g}$. Hence there exists a root $\beta$ of $(\mathfrak{g}, \mathfrak{h})$ such that $\left({ }^{1}\right) H_{\beta} \in \mathfrak{h} \cap \mathfrak{p}=\left\{\right.$ (see [4 (g), Lemma 33]). Let $M_{\mathrm{c}}$ denote the (connected) complex adjoint group of $\mathfrak{m}_{c}$. We can choose $y \in M_{c}$ such that $\mathfrak{h}_{c}{ }^{y}=\mathfrak{a}_{c}$. Then $\alpha=\beta^{y}$ is a root of $(\mathfrak{g}, \mathfrak{a})$ and $H_{\alpha}=\left(H_{\beta}\right)^{y}=H_{\beta} \in \mathfrak{l}$.

Now we know that $\Theta \neq 0$ and by Lemma $59 \Theta(m) \in^{0} \mathrm{~V}(m \in M)$. Therefore it follows from Lemma 66 and the definition of $\Gamma$, that there exists an element $s \in W$ such that $s \lambda=0$ on $\mathfrak{l}$. But then $s \lambda\left(H_{\alpha}\right)=0$ and therefore $\varpi(\lambda)=0$, contrary to our hypothesis. This proves the lemma.

(1) Here $H_{B}$ has the usual meaning (see $[4(\mathrm{n}), \S 4]$ ). 


\section{\$35. Eigenfunctions of $\beta$ in $C(G)$}

Let us now assume that $\operatorname{rank} G=\operatorname{rank} K$ and use the notation of $\S 20$. Let $L^{\prime}$ be the set of all $\lambda \in L$ where $\varpi(\lambda) \neq 0$. We denote by $\chi_{\lambda}\left(\lambda \in L^{\prime}\right)$ the corresponding homomorphism (see [4 (p), § 29]) of 8 into $\mathbf{C}$ so that $z \Theta_{\lambda}=\chi_{\lambda}(z) \Theta_{\lambda}(z \in Z)$. Consider the space $\mathcal{C}_{\lambda}(G)$ of all functions $f \in \mathcal{C}(G)$ such that $z f=\chi_{\lambda}(z) f(z \in 3)$. Let $\mathfrak{S}_{\lambda}$ denote the closure of $\mathcal{C}_{\lambda}(G)$ in $L_{2}(G)$ and $\mathfrak{S}$ the smallest closed subspace of $L_{2}(G)$ containing $\bigcup_{\lambda \in L} \cdot \mathfrak{y}_{\lambda}$.

It is obvious from the definition of $\Theta_{\lambda}$ (see [4 (p), Theorem 3]) that

$$
\Theta_{\lambda}\left(x^{-1}\right)=\operatorname{conj} \Theta_{\lambda}(x)=(-1)^{m} \Theta_{-\lambda}(x) \quad\left(\lambda \in L^{\prime}, x \in G^{\prime}\right),
$$

where $m=\frac{1}{2}$ (dim $\mathfrak{g}-$ rank $\mathfrak{g}$ ). Hence it follows that

$$
\chi_{\lambda}\left(z^{*}\right)=\operatorname{conj} \chi_{\lambda}(\eta(z))=\chi-\lambda(z) \quad(z \in Z),
$$

where $z^{*}$ denotes the adjoint of the differential operator $z$ and $\eta$ the conjugation of $\mathfrak{g}_{c}$ with respect to $\mathrm{g}$.

Lемма 68. Let $f$ be any eigenfunction of 3 in $\mathcal{C}(G)$. Then $f \in C_{\lambda}(G)$ for some $\lambda \in L^{\prime}$.

We may obviously suppose that $f \neq 0$. Let $\chi$ be the homomorphism of 8 into $\mathbf{C}$ such that $z f=\chi(z) f(z \in 3)$. We have to show that $\chi=\chi_{\lambda}$ for some $\lambda \in L^{\prime}$. Suppose this is false. Fix $\lambda \in L$ and consider $\Theta_{\lambda}(f)$. Then

$$
\chi(z) \Theta_{\lambda}(f)=\Theta_{\lambda}(z f)=\chi_{\lambda}\left(z^{*}\right) \Theta_{\lambda}(f)=\chi-\lambda(z) \Theta_{\lambda}(f) \quad(z \in 8) .
$$

Since $\chi \neq \chi_{-\lambda}$, we conclude that $\Theta_{\lambda}(f)=0$. In view of Corollary 2 of Lemma 64, this implies that $f(1)=0$.

Now fix $x \in G$ and put $f_{x}=r(x) f$ in the notation of $\S 10$. Then the above proof is applicable to $f_{x}$ and therefore $f(x)=f_{x}(1)=0$. This shows that $f=0$ and so we get a contradiction. Hence the lemma.

COROLLARY. Let $\phi$ be an element in $L_{2}(G)$ which is an eigendistribution of 3. Then $\phi \in \mathfrak{H}_{\lambda}$ for some $\lambda \in L^{\prime}$.

We may again assume that $\phi \neq 0$. Let $l$ and $r$ respectively denote the left- and rightregular representations of $G$ on $L_{2}(G)$ and $v$ the usual norm on $L_{2}(G)$. For $\alpha, \beta \in C(K)$, define

$$
\alpha * \phi * \beta=\int_{K \times K} \alpha\left(k_{1}\right) \beta\left(k_{2}\right) l\left(k_{1}\right) r\left(k_{2}^{-1}\right) \phi d k_{1} d k_{2}
$$

as usual. Let $\mathcal{L}$ denote the space of all $K$-finite functions in $C(K)$. Since $\mathcal{L}$ is dense in $C(K)$ 6-662900. Acta mathematica. 116. Imprimé lo 10 juin 1966. 
in the norm $|\alpha|_{\infty}=\sup |\alpha|(\alpha \in C(K))$, it follows easily (see $\S 3$ ) that, for any $\varepsilon>0$, we can choose $\alpha, \beta \in \mathcal{L}$ such that

$$
\nu(\phi-\alpha * \phi * \beta)<\varepsilon .
$$

Put $\psi=\alpha * \phi * \beta$ and suppose $\varepsilon<\nu(\phi)$. Then it is clear that $\psi \neq 0$ and $z \psi=\chi(z) \psi(z \in \Re)$. Hence we conclude from Lemma 68 and Corollary 1 of Lemma 65 that $\psi \in C_{\lambda}(G)$ for some $\lambda \in L^{\prime}$. Therefore, in particular, $\chi=\chi_{\lambda}$. Since the space $C_{\lambda}(G)$ depends only on $\chi_{\lambda}$ (and not on $\lambda)$, this shows that $\phi \in \mathrm{Cl}\left(\mathrm{C}_{\lambda}(G)\right)=\mathfrak{S}_{\lambda}$.

\section{§ 36. The role of the distributions $\boldsymbol{\theta}_{\lambda}$ in the harmonic analysis on $G$}

For any $\mathfrak{D} \in \mathcal{E}_{K}$, let $\Theta_{\lambda, \mathfrak{b}}\left(\lambda \in L^{\prime}\right)$ denote the corresponding Fourier component of $\Theta_{\lambda}$ (see [4(q), § 17]).

THEOREM 12. $\Theta_{\lambda, \delta} \in \mathcal{C}_{\lambda}(G)$ for $\lambda \in L^{\prime}$ and $\delta \in \mathcal{E}_{K}$.

This follows from Theorem 9 and Lemma 67 (see also the proof of Corollary 1 of Lemma 65).

Coroliary 1. $\mathcal{C}_{\lambda}(G) \neq\{0\}$ for $\lambda \in L^{\prime}$.

Since $\Theta_{\lambda} \neq 0$, we conclude from Lemma 9 that $\Theta_{\lambda, \delta} \neq 0$ for some $\mathfrak{\delta} \in \mathcal{E}_{K}$. This implies our assertion.

Fix $\lambda_{0} \in L^{\prime}$ and let $L\left(\lambda_{0}\right)$ denote the set of all $\lambda \in L$ of the form $\lambda=s \lambda_{0}(s \in W=W(\mathfrak{g} / \mathfrak{b})$ in the notation of [4(p)]). Let $E_{\lambda_{\theta}}$ denote the orthogonal projection of $L_{2}(G)$ on $\mathfrak{F}_{\lambda_{0}}$ and define

as usual.

$$
(f, g)=\int_{G}(\operatorname{conj} f) g d x \quad\left(f, g \in L_{2}(G)\right)
$$

Let $\mathcal{L}(G)$ denote the space of all $K$-finite functions in $C_{c}^{\infty}(G)$.

Corollary 2. Let $\gamma \in \mathcal{L}(G)$ and $\lambda_{0} \in L^{\prime}$. Then $E_{\lambda_{0}} \gamma \in \mathcal{C}_{\lambda_{0}}(G)$ and

for $\lambda \in L^{\prime}$

$$
\Theta_{\lambda}\left(E_{\lambda_{0}} \gamma\right)=\left\{\begin{array}{cl}
\Theta_{\lambda}(\gamma), & \text { if } \lambda \in L\left(-\lambda_{0}\right), \\
0 & \text { otherwise, }
\end{array}\right.
$$

It is obvious that $E_{\lambda_{0}}$ commutes with the translations of $G$ and therefore $f=E_{\lambda_{0}} \gamma$ is $K$-finite. Hence we conclude from Corollary 1 of Lemma 65 that $f \in \mathcal{C}_{\lambda_{0}}(G)$. Therefore (see the proof of Lemma 68$), \Theta_{\lambda}(f)=0\left(\lambda \in L^{\prime}\right)$ unless $\lambda \in L\left(-\lambda_{0}\right)$. Now fix $\lambda \in L\left(-\lambda_{0}\right)$. Then conj $\Theta_{\lambda, \delta} \in \mathcal{C}_{\lambda_{0}}(G)$ from Theorem 12 and therefore 


$$
\Theta_{\lambda, \mathrm{b}}(\gamma)=\left(\operatorname{conj} \Theta_{\lambda, \mathrm{b}}, \gamma\right)=\left(\operatorname{conj} \Theta_{\lambda, \mathfrak{b}}, E_{\lambda_{0}} \gamma\right)=\Theta_{\lambda_{1} \mathrm{\delta}}(f) \quad\left(\mathfrak{D} \in \mathcal{E}_{K}\right)
$$

from the corollary of Theorem 9 . Therefore, since $\gamma$ and $f$ are both $K$-finite, we have

$$
\Theta_{\lambda}(\gamma)=\sum_{\mathfrak{d}} \Theta_{\lambda, \delta}(\gamma)=\sum_{\mathfrak{d}} \Theta_{\lambda, \delta}(f)=\Theta_{\lambda}(f)
$$

L.тмм 69. Fix $\lambda_{0} \in L^{\prime}$ and define $c$ and $q$ as in Theorem 8. Then

$$
(-1)^{q} \sum_{\lambda \in L\left(-\lambda_{0}\right)} \varpi(\lambda) \Theta_{\lambda}(\ddot{\alpha} * f)=c\left(\alpha, E_{\lambda_{0}} f\right)
$$

for $\alpha \in C_{c}^{\infty}(G)$ and $f \in \mathrm{C}(G)$. Here $\alpha(x)=\operatorname{conj} \alpha\left(x^{-1}\right)(x \in G)$.

Since $\alpha * f \in \mathcal{C}(G)$ (see $\S 10$ ), the left side is defined. Fix $\alpha_{0}, \beta \in \mathcal{L}(G)$ and put $g=E_{\lambda_{0}} \beta$. Then $\bar{\alpha}_{0} * g=E_{\lambda_{0}}\left(\ddot{\alpha}_{0} * \beta\right)$. Now apply Corollary 2 of Lemma 64 to $\tilde{\alpha}_{0} * g$, taking into account Corollary 2 of Theorem 12 with $\gamma=\tilde{\alpha}_{0} * \beta$. Then we get

$$
c\left(\alpha_{0}, E_{\lambda_{0}} \beta\right)=(-1)^{q} \sum_{\lambda \in L\left(-\lambda_{0}\right)} \varpi(\lambda) \Theta_{\lambda}\left(\check{\alpha}_{0} * \beta\right) .
$$

On the other hand, $\mathcal{L}(G)$ is dense both in $C_{c}{ }^{\infty}(G)$ and $\mathcal{C}(G)$, by Lemmas 9,16 and 19 . Moreover, convergence in either one of these spaces implies convergence in $L_{2}(G)$ (see Lemma 11). Finally, if $\alpha_{0}$ and $\beta$ are two variable elements of $\mathcal{L}(G)$, which converge to $\alpha$ and $f$ in $C_{c}^{\infty}(G)$ and $\mathcal{C}(G)$ respectively, then it is obvious from $\S 10$ that $\tilde{\alpha}_{0} * \beta$ tends to $\tilde{\alpha} * f$ in $\mathcal{C}(G)$. Therefore the statement of Lemma 69 now follows immediately.

Define the representation $r$ of $G$ on $C(G)$ as in $\S 10$.

Corollary 1. Let $f \in \mathcal{C}(G)$. Then $E_{\lambda_{0}} f$ is a continuous function on $G$ given by

$$
E_{\lambda_{0}} f(x)=c^{-1}(-1)^{q} \sum_{\lambda \in L\left(-\lambda_{0}\right)} \varpi(\lambda) \Theta_{\lambda}(r(x) f) \quad(x \in G)
$$

It is obvious that the right side is continuous in $x$ and the equality follows from Lemma 69, if we observe that $\Theta_{\lambda}(\tilde{\alpha} * f)=\Theta_{\lambda}(f * \bar{\alpha})\left(\alpha \in C_{c}{ }^{\infty}(G)\right)$, in view of the invariance: of $\Theta_{\lambda}$.

Let $E$ denote the orthogonal projection of $L_{2}(G)$ on $\mathfrak{S}$.

CorolLaRY 2. $c(\alpha, E f)=(-1)^{\alpha} \sum_{\lambda \in L} \varpi(\lambda) \Theta_{\lambda}(\tilde{\alpha} * f)$ for $\alpha \in C_{c}{ }^{\infty}(G)$ and $f \in \mathcal{C}(G)$.

This is obvious from Lemma 69.

Corollary 3. For $f \in \mathrm{C}(G)$, put

$$
f^{\natural}(x)=c^{-1}(-1)^{Q} \sum_{\lambda \in L} \varpi(\lambda) \Theta_{\lambda}(r(x) f) \quad(x \in G) .
$$

Then $f^{\natural}$ is a continuous function on $G$ and $f^{\natural}=E f$. 
Put

$$
T(f)=c^{-1}(-1)^{q} \sum_{\lambda \in L} \varpi(\lambda) \Theta_{\lambda}(f) \quad(f \in \mathcal{C}(G))
$$

We have seen in $\S 20$ that $T$ is a tempered distribution. Hence it is obvious that $f^{\natural}$ is continuous. The rest follows from Corollary 2 , if we take into account the fact that

$$
T(\tilde{\alpha} * f)=T(f * \tilde{\alpha})=\int f^{\natural}(x) \operatorname{conj} \alpha(x) \cdot d x .
$$

Let $W(\lambda)\left(\lambda \in L^{\prime}\right)$ be the set of all $s \in W$ such that $s \lambda \in L$. Put $\left(^{1}\right)$

$$
\Theta_{\lambda}^{*}=[W(\lambda)]^{-1} \sum_{s \in W(\lambda)} \varepsilon(s) \Theta_{s \lambda} \quad\left(\lambda \in L^{\prime}\right)
$$

Corollary 4. For any $\lambda \in L^{\prime}$, the distribution $(-1)^{q} \varpi(\lambda) \Theta_{\lambda}{ }^{*}$ is of positive type.

This is obvious from Lemma 69 since $c\left(\alpha, E_{\lambda} \alpha\right) \geqslant 0$ for $\alpha \in C_{c}^{\infty}(G)$.

LEMMA 70. Fix $\mathrm{D} \in \mathcal{E}_{K}$. Then there exist only a finite number of $\lambda \in L^{\prime}$ such that $\Theta_{\lambda, \S} \neq 0$.

Let $c$ be the center and $g_{1}$ the derived algebra of $g$. Fix a quadratic form $Q$ on $g$ such that 1) $Q$ is negative-definite on $c, 2) \mathfrak{c}$ and $g_{1}$ are mutually orthogonal under $Q$ and 3 ) $Q(X)=\operatorname{tr}(\operatorname{ad} X)^{2}$ for $X \in g_{1}$. Then $Q$ is negative-definite on $\mathrm{f}$, positive-definite on $p$ and it is invariant under $G$. Moreover, $\mathfrak{l}$ and $\mathfrak{p}$ are orthogonal under $Q$. Fix bases $\left(Y_{1}, \ldots, Y_{p}\right)$ and $\left(Z_{1}, \ldots, Z_{q}\right)$ for $\mathfrak{p}$ and $\mathfrak{f}$ wich are orthonormal with respect to $Q$ and $-Q$ respectively and put

$$
\omega_{\mathfrak{p}}=Y_{1}^{2}+\ldots+Y_{p}^{2}, \omega_{\mathfrak{l}}=-\left(Z_{1}^{2}+\ldots+Z_{q}^{2}\right)
$$

in SH. Then

$$
\omega=\omega_{\mathfrak{p}}+\omega_{\mathfrak{l}} \in 8
$$

Let $\gamma$ denote the canonical isomorphism of 8 into $\left({ }^{2}\right) S\left(\mathfrak{b}_{c}\right)=\mathfrak{S}\left(\mathfrak{b}_{c}\right)$ (see [4 (e), Lemma 19]) and $\gamma(z: \mu)(z \in \mathcal{B}, \mu \in \mathfrak{F})$ the value of the polynomial function $\gamma(z)$ at $\mu$. Put

$$
\varrho=\frac{1}{2} \sum_{\alpha \in P} \alpha,
$$

where $P$ is the set of all positive roots of $(\mathfrak{g}, \mathfrak{b})$. Then (see [4 (e), p. 144])

$$
|\mu|^{2}=\gamma(\omega: \mu)+\frac{1}{2} \sum_{\alpha \in P} \varrho\left(H_{\alpha}\right) \quad(\mu \in \mathfrak{F})
$$

is a positive-definite quadratic form on $\mathfrak{F}$ and $\gamma(\omega: \varrho)=0$, so that

$$
\gamma(\omega: \mu)=|\mu|^{2}-|\varrho|^{2}
$$

Moreover, $\chi_{\lambda}(\omega)=\gamma(\omega: \lambda)=|\lambda|^{2}-|\varrho|^{2}$ for $\lambda \in L^{\prime}$.

(1) See the foot-note on p. 3.

(2) Here the notation is the same as in [4 (p)]. 
Let $\mathfrak{K}$ be the subalgebra of $\mathscr{S}$ generated by $\left(1, \mathfrak{F}_{c}\right)$ and $3_{K}$ the center of $\mathfrak{K}$. Then $\omega_{\mathrm{E}} \in \AA_{K}$ and

$$
\omega_{\mathbb{E}} \alpha_{\mathrm{D}}=\chi_{\mathrm{D}}\left(\omega_{\mathrm{E}}\right) \alpha_{\mathrm{D}},
$$

where $\chi_{\triangleright}\left(\omega_{\mathfrak{l}}\right)$ is a number $\geqslant 0$ (see $\S 3$ ). Therefore since $L$ is a lattice in $\mathfrak{F}$, it would be sufficient to prove the following lemma.

LE M M 71. Suppose $\lambda$ and $\delta$ are two elements in $L^{\prime}$ and $\mathcal{E}_{K}$ respectively such that $\Theta_{\lambda, \emptyset} \neq 0$. Then

$$
|\lambda|^{2} \leqslant \chi_{\mathrm{b}}\left(\omega_{\mathfrak{l}}\right)+|\varrho|^{2}
$$

We know from Theorem 12 that $f=\Theta_{\lambda, \diamond} \in C_{\lambda}(G) \subset L_{2}(G)$. Let $r$ denote the right-regular representation of $G$ on $L_{2}(G), V$ the smallest closed subspace of $L_{2}(G)$ containing $f$ which is invariant under $r$, and $\pi$ the restriction of $r$ on $V$. Since convergence in $\mathcal{C}(G)$ implies convergence in $L_{2}(G)$, it follows from Lemma 15 that $f$ is differentiable under $\pi$ and

$$
\pi(\omega) f=\chi_{\lambda}(\omega) f, \quad \pi\left(\omega_{t}\right) f=\chi_{\mathrm{D}}\left(\omega_{\mathrm{t}}\right) f .
$$

Hence

$$
\left\{|\lambda|^{2}-|\varrho|^{2}\right\}\|f\|^{2}=(f, \pi(\omega) f)=\chi_{\triangleright}\left(\omega_{\mathfrak{q}}\right)\|f\|^{2}+\left(f, \pi\left(\omega_{\mathfrak{p}}\right) f\right),
$$

where \|\| denotes the usual norm in $L_{2}(G)$. But since $\pi$ is unitary and $f$ is differentiable under $\pi$, it is obvious that

$$
\left(f, \pi\left(\omega_{\mathfrak{p}}\right) f\right)=-\sum_{1 \leqslant i \leqslant p}\left\|\pi\left(Y_{i}\right) f\right\|^{2} \leqslant 0 .
$$

Hence our assertion follows immediately from the fact that $\|f\|>0$.

Lемма 72. Let $f$ be a $K$-finite function in $\mathcal{C}(G)$. Then $E_{\lambda} f \in \mathcal{C}_{\lambda}(G)\left(\lambda \in L^{\prime}\right)$ and $E_{\lambda} f=0$ for all $\lambda \in L^{\prime}$ except a finite number. Hence $E f \in \mathcal{C}(G)$ and it is both $K$-finite and 3-finite.

Since $E_{\lambda}$ commutes with the translations of $G$, it is clear that $E_{\lambda} f$ is $K$-finite and therefore, by Corollary 1 of Lemma 65 , it lies in $\mathcal{C}_{\lambda}(G)$. Now select a finite subset $F$ of $\mathcal{E}_{K}$ such that $f=\alpha_{F} * f * \alpha_{F}$ in the notation of $\S 12$. Replacing $F$ by $F \cup F^{*}$, we may assume that $F=F^{*}$. (Recall that $\delta^{*}$ is the class in $\mathcal{E}_{K}$ contragradient to $\mathfrak{b}$.) Put

$$
f_{\lambda}(x)=\Theta_{\lambda}(r(x) f) \quad(x \in G)
$$

for $\lambda \in L^{\prime}$. Then it is obvious that

$$
f_{\lambda}(x)=\Theta_{\lambda, F}(r(x) f),
$$

where

$$
\Theta_{\lambda, F}=\sum_{\delta \in F} \Theta_{\lambda, \delta}
$$


Hence we conclude from Lemma 70 that $f_{\lambda}=0$ for all $\lambda \in L^{\prime}$ except a finite number, and therefore the assertions of the lemma follow from Corollaries 1 and 3 of Lemma 69.

Define $B^{\prime}=B \cap G^{\prime}$ as in $\S 19$.

Lемма 73. Fix $\lambda \in L^{\prime}$ and let $\Theta$ be a tempered and invariant distribution on $G$ such that $z^{*}=\chi_{\lambda}(z) \Theta(z \in 3)$. Then $\Theta_{\triangleright} \in \mathcal{C}_{\lambda}(G)$ for $\downarrow \in \mathcal{E}_{K}$. Moreover, in order to show that $\Theta=0$, it is sufficient to verify either one of the following two conditions.

1) $\Theta(f)=0$ for every $K$-finite function $f \in \mathcal{C}_{-\lambda}(G)$.

2) $\Theta=0$ pointwise $\left(^{1}\right)$ on $B^{\prime}$.

It follows from Theorem 9 and Lemma 67 that $\Theta_{\triangleright} \in \mathcal{C}_{\lambda}(G)\left(\delta \in \mathcal{E}_{R}\right)$ and therefore $f=\operatorname{conj} \Theta_{\S} \in \mathcal{C}_{-\lambda}(G)$ (see $\S 35$ ). Then

$$
\Theta_{\mathfrak{b}}(f)=\int_{G}\left|\Theta_{\mathfrak{b}}(x)\right|^{2} d x
$$

from the corollary of Theorem 9. Therefore $\Theta_{\mathfrak{b}}=0$ under condition 1). In view of Lemma 9, this implies that $\Theta=0$.

On the other hand, by Lemma 64 and the corollary of Theorem 7,2) implies 1) and so the lemma is proved.

Define $W_{G}$ as in [4 (p), Theorem 3]. Then $L$ is stable under $W_{G}$ and $\Theta_{s \lambda}=\varepsilon(s) \Theta_{\lambda}$ $\left(s \in W_{G}, \lambda \in L^{\prime}\right)$.

LEMMA 74. Let $\Theta$ be an invariant eigendistribution of 3 on $G$, which is tempered. Suppose there exists an element $\mathfrak{\delta} \in \mathcal{E}_{K}$ such that $\Theta_{\mathfrak{D}} \neq 0$ and $\Theta_{\triangleright} \in L_{2}(G)$. Then we can choose $\lambda \in L^{\prime}$ such that $z \Theta=\chi_{\lambda}(z) \Theta(z \in 3)$. Moreover, for any such $\lambda$, there exist unique complex numbers $c_{s}(s \in W(\lambda))$ such that $c_{t s}=c_{s}\left(t \in W_{G}\right)$ and

$$
\Theta=\left[W_{G}\right]^{-1} \sum_{s \in W(\lambda)} \varepsilon(s) c_{s} \Theta_{s \lambda} .
$$

The first statement follows from the corollary of Lemma 68 . Now put

$$
\Phi(b)=\Delta(b) \Theta(b) \quad\left(b \in B^{\prime}\right),
$$

where $\Delta$ has the usual meaning (see [4 (p), Theorem 3]). Then it follows from [4 (o), Lemma 31] that $\Phi$ extends to an analytic function on $B$. Moreover, we know from [4 (e), Theorem 2] that

$$
\gamma(z) \Phi=\chi_{\lambda}(z) \Phi \quad(z \in 8)
$$

(1) Here we have to make use of Theorem 2 of [4 (0)]. 
where $\gamma(z) \in \mathfrak{S}_{\left(\mathfrak{b}_{c}\right)}$ is to be regarded as a differential operator on $B$. Finally it is obvious that $\Phi\left(b^{s}\right)=\varepsilon(s) \Phi(b)$ for $s \in W_{G}$ and $b \in B$. Therefore (see [4 (f), p. 102] and [4 (p), Theorem $3]$ ), we can choose unique complex numbers $c_{s}(s \in W(\lambda))$ such that $c_{t s}=c_{s}\left(t \in W_{G}\right)$ and the distribution

$$
\Theta^{\prime}=\Theta-\left[W_{G}\right]^{-1} \sum_{s \in W(\lambda)} \varepsilon(s) c_{s} \Theta_{s \lambda}
$$

vanishes pointwise on $B^{\prime}$. It is clear (see $\S 20$ ) that $\Theta^{\prime}$ is tempered and $z \Theta^{\prime}=\chi_{\lambda}(z) \Theta^{\prime}(z \in \supsetneqq)$. Therefore $\Theta^{\prime}=0$ from Lemma 73 .

Lемма 75. Let $\delta_{0}$ denote the class of the trivial representation of $K$. Then $\Theta_{\lambda, b_{0}}=0$ for $\lambda \in L^{\prime}$.

Fix $\lambda \in L^{\prime}$ and put $\Phi_{\lambda}=\Theta_{\lambda . b_{0}}$. Then from Theorem 9 , there exist numbers $c, m \geqslant 0$ such that

$$
\left|\Phi_{\lambda}\right| \leqslant c \Xi(\mathbf{1}+\sigma)^{m}
$$

This is the analogue of [4 (q), Lemma 43]. By making use of the corollary of [4 (q), Lemma 47], we prove in the same way as in $[4(q), \S 20]$ that $\Phi_{\lambda}=0$.

\section{§ 37. The discrete series for $G$}

Let $G$ be a locally compact unimodular group satisfying the second axiom of countability. Fix a Haar measure $d x$ on $G$. By a unitary representation of $G$, we mean a representation of $G$ on a Hilbert space, which is unitary. Let $\mathcal{E}$ be the set of all equivalence classes of irreducible unitary representations of $G$.

Let $\pi$ be an irreducible unitary representation of $G$ on a Hilbert space $\mathfrak{H}$. We say that $\pi$ is square-integrable if any one of the following two mutually equivalent conditions holds (see [7, p. 640]).

(1) There exist nonzero elements $\phi$, $\psi$ in $\mathfrak{S}$ such that

$$
\int_{G}|(\phi, \pi(x) \psi)|^{2} d x<\infty
$$

(2) There exists a closed subspace $V$ of $L_{2}(G)$ stable under the right-regular representation $r$ of $G$ on $L_{2}(G)$, such that $\pi$ is equivalent to the restriction of $r$ on $V$.

It is known (see $[7$, p. 640]) that, if $\pi$ is square-integrable, there exists a number $d(\pi)>0$ such that

$$
\int_{G}|(\phi, \pi(x) \psi)|^{2} d x=d(\pi)^{-1}\|\phi\|^{2}\|\psi\|^{2} \quad(\phi, \psi \in \mathfrak{S})
$$


where $\|\phi\|$ is the norm of $\phi$ in $\mathfrak{H}$. We shall call $d(\pi)$ the formal degree of $\pi$ (see [4 (d), §3]). It is obvious that square-integrability, as well as the formal degree, are invariant under equivalence. We call a class $\omega \in \mathcal{E}$ discrete if every representation $\pi \in \omega$ is square-integrable and put $d(\omega)=d(\pi)$.

Let $\mathcal{E}_{d}$ denote the set of all discrete classes in $\mathcal{E}$. Then $\mathcal{E}_{d}$ is called the discrete series of $G$.

Now let us return to the case when $G$ and $K$ are defined as in $\S 7$. For any $w \in \mathcal{E}$, let $\Theta_{\omega}$ denote the character (see $[4(\mathrm{~b}), \S 5]$ ) and $\chi_{\omega}$ the infinitesimal character of $\omega$ so that $z \Theta_{\omega}=\chi_{\omega}(z) \Theta_{\omega}(z \in Z)$.

L IMмA 76. Let $\omega \in \mathcal{E}_{d}$. Then $\Theta_{\omega}$ is tempered and $\Theta_{\omega, \mathrm{D}} \in L_{2}(G)$ for $\delta \in \mathcal{E}_{K}$.

Fix $\pi \in \omega$ and let $\mathfrak{H g}$ be the representation space of $\pi$. We now use the notation of $\S 25$ and put

Then $\quad \Theta_{\omega}(f)=\sum_{i \in J} \int f \phi_{i} d x \quad\left(f \in C_{c}^{\infty}(G)\right)$,

the series being absolutely convergent (see [4 (b), p. 243]). Moreover, $\phi_{i}$ is analytic from [4 (q), Lemma 33]. Fix an integer $m \geqslant 0$ as in Lemma 7. Then

$$
\int f \phi_{i} d x=c(\mathfrak{D})^{-m} \int f \cdot \Omega^{m} \phi_{i} d x=c(\mathfrak{D})^{-m} \int \Omega^{m} f \cdot \phi_{i} d x \quad\left(i \in J_{\mathfrak{D}}\right),
$$

in the notation of Lemma 6. Hence, by the Schwartz inequality, we get

$$
\left|\int f \phi_{i} d x\right| \leqslant c(\delta)^{-m}\left\|\Omega^{m} f\right\| d(\pi)^{-\frac{1}{b}} \quad\left(i \in J_{\triangleright}\right),
$$

where \|\| denotes the usual norm in $L_{2}(G)$. This shows that

$$
\left|\Theta_{\omega}(f)\right| \leqslant d(\omega)^{-\frac{1}{2}}\left\|\Omega^{m} f\right\|_{\mathfrak{D} \in \mathcal{E}_{\underline{K}}} c(\mathfrak{D})^{-m} \operatorname{dim} \mathfrak{H}_{\mathfrak{b}} \quad\left(f \in C_{c}^{\infty}(G)\right) .
$$

But

$$
\sum_{\mathbb{D}} c(\mathfrak{D})^{-m} \operatorname{dim} \mathfrak{S}_{\mathfrak{D}} \leqslant N \sum_{\mathfrak{D}} c(\mathfrak{D})^{-m} d(\mathfrak{D})^{2}<\infty
$$

from Lemma 7. Since

$$
\nu(g)=\left\|\Omega^{m} g\right\| \quad(g \in \mathbb{C}(G))
$$

is a continuous seminorm on $\mathcal{C}(G)$ (see Lemma 11), we conclude that $\Theta_{\omega}$ is tempered.

Moreover, (see § 25),

and therefore $\Theta_{\omega, \mathrm{D}} \in L_{2}(G)$.

$$
\Theta_{\omega, \mathfrak{b}}=\sum_{i \in J_{\mathbb{D}}} \phi_{i} \quad\left(\delta \in \mathcal{E}_{E}\right)
$$




\section{§ 38. Proof of Lemma 65}

As before, let $r$ denote the right-regular representation of $G$ on $L_{2}(G)$.

LEM M A7. Suppose $f \neq 0$ is a 3-finite function in $L_{2}(G)$ such that the right translates of $f$, under $K$, span a finite-dimensional space. Let $V$ be the smallest closed subspace of $L_{2}(G)$ containing $f$, which is stable under $r$. Then

$$
V=\sum_{1 \leqslant i \leqslant p} U_{i}
$$

where $U_{i}$ are mutually orthogonal closed subspaces of $V$, which are invariant and irreducible under $r$.

Let $\pi$ denote the restriction of $r$ on $V$. For any finite subset $F$ of $\mathcal{E}_{K}$, define $E_{F}$ as in $\S 25$ and put $V_{F}=E_{F} V$. It is clear that $F^{\prime}$ can be so chosen that $f \in V_{F}$. Define $V^{\infty}$ as in Lemma 4. We know from [4 (q), Lemma 33] that $f$ is analytic and therefore, by Theorem 1 , $f=f * \alpha$ for some $\alpha \in C_{c}{ }^{\infty}(G)$. This shows (Lemma 2) that $f \in V^{\infty}$. Moreover, a simple argument (see $\S 8$ ) shows that $W=\pi((6)) f$ is dense in $V$. Finally we conclude from [4 (a), Theorem 1] that

$$
W=\sum_{D \in \varepsilon_{K}} E_{\triangleright} W
$$

and $W_{F}=E_{F} W$ has finite dimension. Since $W_{F}$ is dense in $V_{F}$, it follows that $W_{F}=V_{F}$.

Let $U \neq\{0\}$ be any closed subspace of $V$ stable under $\pi$. We claim that $E_{F} U \neq\{0\}$. For otherwise suppose $E_{F} U=\{0\}$. Let $U^{\prime}$ denote the orthogonal complement of $U$ in $V$. Since $\pi$ is unitary, $U^{\prime}$ is also stable under $\pi$ and $f \in V_{F} \subset U^{\prime}$. But this implies that $V \subset U^{\prime}$ and therefore $U=\{0\}$, contradicting our hypothesis.

Let $U_{i}(1 \leqslant i \leqslant p)$ be a finite set of mutually orthogonal, closed, nonzero subspaces of $\mathrm{V}$, which are stable under $\pi$. Then since $U_{i} \cap V_{F} \neq\{0\}$, we conclude that $p \leqslant \operatorname{dim} V_{F}<\infty$. Therefore the required result follows immediately by assuming that $p$ has the largest possible value.

Now we come to the proof of Lemma 65. We may assume that $f \neq 0$. Define $V$ and $U_{i}$ $(1 \leqslant i \leqslant p)$ as in Lemma 77 and put $f_{i}=E_{i} f$, where $E_{i}$ is the orthogonal projection of $V$ on $U_{i}$. It is obvious that $f_{i}$ is 8 -finite as well as $K$-finite. Hence by [ 4 (q), Lemma 33], it is analytic. Moreover, $f=f_{1}+\ldots+f_{p}$. Therefore it would be sufficient to prove Lemma 65 for each $f_{i}$. Thus we may assume that $V$ is irreducible under $\pi$ so that $\pi$ is square-integrable. By Theorem 1, there exists an element $\alpha \in C_{c}{ }^{\infty}(G)$ such that $f=\alpha * f$. Since $f$ is $K$-finite, we can obviously assume that $\alpha$ is also $K$-finite. But then

$$
f(x)=(\tilde{\alpha}, \pi(x) f)=(E \ddot{\alpha}, \pi(x) f) \quad(x \in G)
$$

where $\tilde{\alpha}$ is defined as in Lemma 69 and $E$ is the orthogonal projection of $L_{2}(G)$ on $V$. The required inequality is now an immediate consequence of Theorem 10 and Lemma 76. 


\section{§ 39. The existence of the discrete series}

Henceforward we assume, for convenience, that $G$ is acceptable.

THEOREM 13. $G$ has a discrete series if and only if $\operatorname{rank} G=\operatorname{rank} K$.

Suppose $\mathcal{E}_{d} \neq \varnothing$. Fix $\omega \in \mathcal{E}_{d}$ and choose $\mathfrak{D} \in \mathcal{E}_{K}$ such that $f=\Theta_{\omega, \emptyset} \neq 0$. Then it follows from Lemma 76 and Corollary 2 of Lemma 65 that rank $G=\operatorname{rank} K$.

Conversely suppose $\operatorname{rank} G=\operatorname{rank} K$. Fix $\lambda \in L^{\prime}$ and choose $\delta \in \mathcal{E}_{K}$ such that $\Theta_{\lambda, \delta} \neq 0$. Then by Theorem 12, the function $f=\Theta_{\lambda .0}$ satisfies the hypotheses of Lemma 77. Let $\pi_{i}$ denote the restriction of $r$ on $U_{i}(1 \leqslant i \leqslant p)$, in the notation of Lemma 77. Then $\pi_{i}$ is squareintegrable and therefore $\mathcal{E}_{d} \neq \varnothing$.

\section{\$ 40. The characters of the discrete series}

In view of Theorem 13, we shall now assume that $\operatorname{rank} G=\operatorname{rank} K$ and use the notation of $\S \S 36,37$.

For any $\lambda \in L^{\prime}$, let $\mathcal{E}_{d}(\lambda)$ denote the set of all $\omega \in \mathcal{E}_{d}$ such that $\chi_{\omega}=\chi_{\lambda}$. The following result is an immediate consequence of Lemmas 74 and 76.

L匹MмA 78. $\mathcal{E}_{d}=\bigcup_{\lambda \in L^{\prime}} \cdot \mathcal{E}_{d}(\lambda)$. Moreover, for any $\omega \in \mathcal{E}_{d}(\lambda)\left(\lambda \in L^{\prime}\right)$, there exist unique complex numbers $c_{s}(\omega)(s \in W(\lambda))$ such that $c_{t s}(\omega)=c_{s}(\omega)\left(t \in W_{G}\right)$ and

$$
\Theta_{\omega}=\left[W_{G}\right]^{-1} \sum_{s \in W(\lambda)} \varepsilon(s) c_{s}(\omega) \Theta_{s \lambda}
$$

For any $\omega \in \mathcal{E}$, define the analytic function $\Phi_{\omega}$ on $B$ (see $\S 36$ ) by

$$
\Phi_{\omega}(b)=\Delta(b) \Theta_{\omega}(b) \quad\left(b \in B^{\prime}\right)
$$

and put

$$
F_{f}(b)=\Delta(b) \int_{G} f\left(b^{x}\right) d x \quad\left(b \in B^{\prime}\right)
$$

for $f \in \mathrm{C}(G)$ as in $\S 18$, Put $G_{B}=\left(B^{\prime}\right)^{G}$ and let $d b$ denote the normalized Haar measure on $B$. Then $G_{B}$ is open in $G$ and (see Lemma 91)

$$
\int_{G} \alpha(x) d x=(-1)^{m}\left[W_{G}\right]^{-1} \int_{B} \Delta F_{\alpha} d b \quad\left(\alpha \in C_{c}^{\infty}\left(G_{B}\right)\right),
$$

where $m=\frac{1}{2} \operatorname{dim} G / B$.

Lемма 79. Let $f$ be a B-finite function in $\mathrm{C}(G)$. Then

$$
\Theta_{\omega}(f)=(-1)^{m}\left[W_{G}\right]^{-1} \int_{B} F_{f} \Phi_{\omega} d b \quad\left(\omega \in \mathcal{E}_{d}\right)
$$


This follows immediately from Lemmas 64 and 76 and the corollary of Theorem 7 .

LEMMA 80. Let $\pi$ be a square-integrable representation of $G$ on $\mathfrak{Y}$ and $\omega$ its class in $\mathcal{E}_{d}$. Fix two K-finite elements $\phi, \psi \in \mathfrak{H}$ and put

$$
f(x)=(\phi, \pi(x) \psi) \quad(x \in G)
$$

Then

$$
F_{f}=d(\omega)^{-1}(\phi, \varphi) \Phi_{\omega}
$$

on $B^{\prime}$.

First observe that $f \in \mathrm{C}(G)$ from Corollary 1 of Lemma 65 and therefore $F_{f}$ is defined. Now fix $\alpha \in C_{c}^{\infty}(G)$ and consider the operator

$$
\pi(\alpha)=\int \alpha(x) \pi(x) d x
$$

Then $\Theta_{\omega}(\alpha)=\operatorname{tr} \pi(\alpha)$ and the argument of $[4(d), 576]$ shows that

$$
\int_{G} d x \int_{G} \alpha(y) f\left(y^{x}\right) d y=\int_{G}\left(\phi, \pi(x) \pi(\alpha) \pi\left(x^{-1}\right) \psi\right) d x=d(\omega)^{-1}(\phi, \psi) \Theta_{\omega}(\alpha) .
$$

Now suppose $\alpha \in C_{c}^{\infty}\left(G_{B}\right)$. Then we claim that

$$
\int\left|\alpha(y) f\left(y^{x}\right)\right| d x d y<\infty
$$

For we can choose $\alpha_{0} \in O_{c}^{\infty}\left(G_{B}\right)$ such that $\alpha_{0} \geqslant|\alpha|$. Then

$$
\int\left|\alpha(y) f\left(y^{x}\right)\right| d x d y \leqslant \int \alpha_{0}(y)\left|f\left(y^{x}\right)\right| d x d y=\left[W_{G}\right]^{-1} \int_{B}\left|F_{\alpha_{0}}(b)\right|\left\{|\Delta(b)| \int_{G}\left|f\left(b^{y}\right)\right| d y\right\} d b .
$$

Since $\left|F_{\alpha_{0}}\right|$ is bounded on $B^{\prime}$ (Lemma 26), our assertion follows from Theorem 5 .

Therefore we conclude from Fubini's theorem that

$$
d(\omega)^{-1}(\phi, \psi) \Theta_{\omega}(\alpha)=\int \alpha(y) d y \int f\left(y^{x}\right) d x \quad\left(\alpha \in C_{c}^{\infty}\left(G_{B}\right)\right)
$$

But it is clear that

$$
\int \alpha(y) d y \int f\left(y^{x}\right) d x=(-1)^{m}\left[W_{G}\right]^{-1} \int_{B} F_{\alpha} F_{f} d b
$$

and

$$
\Theta_{\omega}(\alpha)=\int \alpha \Theta_{\omega} d x=(-1)^{m}\left[W_{G}\right]^{-1} \int_{B} F_{\alpha} \Phi_{\omega} d b
$$


This shows that

$$
\int F_{\alpha} F_{f} d b=d(\omega)^{-1}(\phi, \psi) \int F_{\alpha} \Phi_{\omega} d b \quad\left(\alpha \in C_{c}{ }^{\infty}\left(G_{B}\right)\right)
$$

On the other hand, it is easy to verify (see $[4(0), \S 20]$ ) that there exists a $C^{\infty}$ function $u$ on $G_{B}$ such that

$$
\Delta(b) u\left(b^{x}\right)=F_{f}(b)-d(\omega)^{-1}(\phi, \psi) \Phi_{\omega}(b) \quad\left(b \in B^{\prime}, x \in G\right)
$$

Then if follows from the above result that

$$
\int \alpha u d x=0 \quad\left(\alpha \in C_{c}^{\infty}\left(G_{B}\right)\right)
$$

and therefore $u=0$. This implies the assertion of the lemma.

For any $\omega \in \mathcal{E}_{d}$, we define a subspace $\mathfrak{H}_{\omega}$ of $L_{2}(G)$ as follows. Fix $\pi \in \omega$ and let $U$ be the representation space of $\pi$. Then $\mathfrak{S}_{\omega}$ is the smallest closed subspace of $L_{2}(G)$ containing all functions $f$ of the form

$$
f(x)=(\phi, \pi(x) \psi) \quad(x \in G)
$$

where $\phi, \psi \in U$. It is clear that this definition is independent of the particular choice of $\pi$ and $\mathfrak{H}_{\omega}$ is stable under both left and right translations of $G$. Put $\mathcal{C}_{\omega}(G)=\mathfrak{S}_{\omega} \cap \mathcal{C}(G)$. Then it follows from Lemma 11 that $C_{\omega}(G)$ is closed in $C(G)$.

THEOREM 14. $\mathcal{C}_{\omega}(G)$ is dense in $\mathfrak{g}_{\omega}$ and

for $f \in \mathcal{C}_{\omega}(G)$ and $\omega \in \mathcal{E}_{d}$

$$
F_{f}=d(\omega)^{-1} f(1) \Phi_{\omega}
$$

Choose an orthonormal base $\psi_{i}(i \in J)$ for $U$ as in $\S 25$ so that $\psi_{i}\left(i \in J_{\mathfrak{b}}\right)$ is a base for $U_{\mathfrak{\delta}}\left(\delta \in \mathcal{E}_{K}\right)$ and put

$$
f_{t j}(x)=\left(\psi_{i}, \pi(x) \psi_{j}\right) \quad(x \in G)
$$

Then it follows from Corollary 1 of Lemma 65 that $f_{i j} \in \mathcal{C}_{\omega}(G)$. This shows that $\mathcal{C}_{\omega}(G)$ is dense in $\mathfrak{H}_{\omega}$.

Let $V$ be the set of all $f \in C_{\omega}(G)$ such that

$$
F_{f}=d(\omega)^{-1} f(1) \Phi_{\omega}
$$

Then it is clear that $V$ is a closed subspace $\left(^{1}\right)$ of $C_{w}(G)$. Hence it would be enough to show that $V$ is dense in $C_{\omega}(G)$.

(1) The topology of $\mathrm{C}_{\omega}(G)$ is the one inherited from $\mathrm{C}_{(G) \text {. }}$ 
Fix $f_{0} \in \mathrm{C}_{\omega}(G)$. Then in view of Lemma 16, it would be enough to prove that $f=\alpha_{D_{1}} * f_{0} * \alpha_{\delta_{3}} \in V$ for $\delta_{1}, \delta_{2} \in \mathcal{E}_{K}$. Since $J_{\searrow}$ is a finite set for every $\delta \in \mathcal{E}_{K}$, it is clear that $f$ is a finite linear combination of $f_{i j}(i, j \in J)$ and therefore $f \in V$ from Lemma 80. This proves the theorem.

Remark. The above proof shows that $f_{i j}(i, j \in J)$ span a dense subspace of $\mathcal{C}_{\omega}(G)$. Moreover, $f_{i l}(1)=1$ for $i \in J$.

For any $w \in \mathcal{E}$, let $\omega^{*}$ denote the class contragredient to $\omega$. It is clear that $\Theta_{\omega *}=\operatorname{conj} \Theta_{\omega}$ as functions on $G$, and $\omega^{*}$ is discrete whenever $\omega$ is discrete.

LEMMA 81. Let $\omega, \omega^{\prime}$ be two elements in $\mathcal{E}_{d}$. Then

for $f \in \mathrm{C}_{\omega},(G)$

$$
\Theta_{\omega}(f)=\left\{\begin{array}{cl}
d(\omega)^{-1} f(1) & \text { if } \omega^{\prime}=\omega^{*}, \\
0 & \text { otherwise, }
\end{array}\right.
$$

We keep to the above notation. Then it follows easily from Lemma 19 that

$$
\Theta_{\omega}(f)=\sum_{f} \int f f_{i i} d x=\sum_{i}\left(\operatorname{conj} f_{i i}, f\right)
$$

for any $K$-finite function $f$ in $\mathcal{C}_{\omega^{\prime}}(G)$. Now conj $f_{i i} \in \mathfrak{W}_{\omega *}$ and if $\omega^{*} \neq \omega^{\prime}$, we conclude from the Schur orthogonality relations [4(d), Theorem 1] that $\mathfrak{S}_{\omega *}$ is orthogonal to $\mathfrak{S}_{\omega^{*}}$ and therefore $\Theta_{\omega}(f)=0$. Since $K$-finite functions are dense in $\mathcal{C}_{\omega^{\prime}}(G)$ by Lemma 16, we get the required assertion in this case.

Now suppose $\omega^{*}=\omega^{\prime}$ and $f=\operatorname{conj} f_{i j}(i, j \in J)$. Then it follows again from the Schur orthogonality relations that

$$
\Theta_{\omega}(f)=d(\omega)^{-1} f(1)
$$

But we have seen above that conj $f_{i j}(i, j \in J)$ span a dense subspace of $C_{\omega *}(G)$ and so the assertion of the lemma is now obvious.

As before, let $d b$ denote the normalized Haar measure of $B$.

Corollary 1.

$$
\int_{B}\left(\operatorname{conj} \Phi_{\omega}\right) \Phi_{\omega^{\prime}} d b=\left\{\begin{array}{c}
{\left[W_{G}\right] \text { if } \omega=\omega^{\prime}} \\
0 \text { otherwise }
\end{array}\right.
$$

Fix $f \in \mathcal{C}_{\omega}(G)$ such that $f(1) \neq 0$ (see the remark after Theorem 14) and put $g=$ conj $f$. Then

$$
\Theta_{\omega^{\prime}}(g)=(-1)^{m}\left[W_{G}\right]^{-1} \int_{B} F_{D} \Phi_{\omega^{\prime}} d b
$$




$$
F_{g}=(-1)^{m} \operatorname{conj} F_{f}
$$

and therefore our assertion follows from Theorem 14 and Lemma 81.

Define the number $c>0$ by the relation (see Lemma 38)

$$
F_{f}(1 ; \omega)=(-1)^{a} c f(1) \quad(f \in \mathcal{C}(G))
$$

Then $c$ has the same value as in Theorem 8 (see $[4(q), \S 15])$. Fix $\lambda \in L^{\prime}$ and for any $\omega \in \mathcal{E}_{d}(\lambda)$, define $c_{s}(\omega)(s \in W(\lambda))$ as in Lemma 78. Then

$$
\Theta_{\omega}=\sum_{s \in W \backslash \backslash W(\lambda)} \varepsilon(s) c_{s}(\omega) \Theta_{s \lambda}
$$

where the sum is over a complete system of representatives.

Corollary 2. Let $\omega \in \mathcal{E}_{d}(\lambda)$. Then

$$
d(\omega)=(-1)^{q} c^{-1} \approx(\lambda) \sum_{s \in W(\lambda)} c_{s}(\omega)
$$

and

$$
\sum_{s \in W(T W(\lambda)}\left|c_{s}(\omega)\right|^{2}=1
$$

We know from Theorem 14 that

$$
(-1)^{q} c f(1)=F_{f}(1 ; \varpi)=d(\omega)^{-1} f(1) \Phi_{\omega}(1 ; \varpi) \quad\left(f \in \mathcal{C}_{\omega}(G)\right)
$$

and the first relation is an immediate consequence of this fact. The second follows by putting $\omega^{\prime}=\omega$ in Corollary 1 above.

Let $\mathfrak{I}(\lambda)\left(\lambda \in L^{\prime}\right)$ be the space of all tempered and invariant distributions $\Theta$ on $G$ such that $z \Theta=\chi_{\lambda}(z) \Theta(z \in \Omega)$.

THEOREM 15. Fix $\lambda \in L^{\prime}$. Then $\Theta_{\omega}\left(\omega \in \mathcal{E}_{d}(\lambda)\right)$ form a base for $\mathfrak{T}(\lambda)$ over $\mathbf{C}$ and

$$
\left[\mathcal{E}_{d}(\lambda)\right]=\operatorname{dim} \mathfrak{T}(\lambda)=[W(\lambda)]\left[W_{G}\right]^{-1}
$$

Moreover,

$$
\sum_{\omega \in \varepsilon_{d}(\lambda)} d(\omega) \Theta_{\omega}=(-1)^{q} c^{-1} \sum_{s \in W(\lambda)} \varpi(s \lambda) \Theta_{s \lambda}
$$

where $c$ and $q$ have the same meaning as in Theorem 8.

We know from Lemma 76 that $\Theta_{\omega}\left(\omega \in \mathcal{E}_{d}(\lambda)\right)$ lie in $\mathfrak{T}(\lambda)$ and from Lemma 81 that they are linearly independent. Now fix $\Theta \in \mathfrak{T}(\lambda)$. We have to show that $\Theta$ is a linear combination of $\Theta_{\omega}\left(\omega \in \mathcal{E}_{\alpha}(\lambda)\right)$. Define analytic functions $\Phi$ and $\Phi_{0}$ on $B$ as follows.

$$
\Phi(b)=\Delta(b) \Theta(b) \quad\left(b \in B^{\prime}\right)
$$


and

$$
\Phi_{0}=\Phi-\sum_{\omega \in \mathcal{E}_{d}(\lambda)} c(\omega) \Phi_{\omega}
$$

where

$$
c(\omega)=\left[W_{G}\right]^{-1} \int_{B} \Phi \operatorname{conj} \Phi_{\omega} d b
$$

Then it is clear from Corollary 1 of Lemma 81 that $\Phi_{0}$ is orthogonal to $\Phi_{\omega}\left(\omega \in \mathcal{E}_{d}(\lambda)\right)$ in $L_{2}(B)$. Put

$$
\Theta_{0}=\Theta-\sum_{\omega \in \varepsilon_{d}(\lambda)} c(\omega) \Theta_{\omega}
$$

We claim that $\Theta_{0}=0$. In view of Lemma 73, it would be enough to verify that $\Theta_{0}(f)=0$ for any $K$-finite function $f \in \mathcal{C}_{-\lambda}(G)$. We may obviously assume that $f \neq 0$. Define $V$ and $U_{i}(1 \leqslant i \leqslant p)$ as in Lemma 77 and let $E_{i}$ denote the orthogonal projection of $V$ on $U_{i}$. Put $f_{i}=E_{i} f(1 \leqslant i \leqslant p)$. Then it follows from Corollary 1 of Lemma 65 that $f_{i} \in \mathcal{C}_{-\lambda}(G)$. Therefore since

$$
\Theta_{0}(f)=\sum_{1 \leqslant i \leqslant q} \Theta_{0}\left(f_{i}\right)
$$

it would be enough to consider the case when $V$ is irreducible under $r$.

Let $\pi$ denote the restriction of $r$ on $V$ and $\omega$ the class in $\mathcal{E}_{d}$ such that $\pi \in \omega^{*}$. Then $\omega \in \mathcal{E}_{d}(\lambda)$ and, as we have seen in $\S 38$, there exists an element $\alpha \in C_{c}{ }^{\infty}(G)$ such that

$$
f(x)=(\alpha, \pi(x) f)=(E \alpha, \pi(x) f) \quad(x \in G) .
$$

(Here $E$ denotes the orthogonal projection of $L_{2}(G)$ on $V$.) This shows that $f \in C_{\omega^{*}}(G)$. On the other hand, it follows from Lemma 64 and the corollary of Theorem 7, that

$$
\Theta_{0}(f)=(-1)^{m}\left[W_{G}\right]^{-1} \int_{B} F_{f} \Phi_{0} d b
$$

Therefore we conclude from Theorem 14 and the definition of $\Phi_{0}$ that $\Theta_{0}(f)=0$.

Let $s_{1}, s_{2}, \ldots, s_{p}$ be a complete set of representatives of $W_{G} \backslash W(\lambda)$ in $W(\lambda)$. Then by Lemma 74, the distributions $\Theta_{s_{i} \lambda}(1 \leqslant i \leqslant \lambda)$ also form a base for $\mathfrak{I}(\lambda)$ and therefore

$$
[W(\lambda)]\left[W_{G}\right]^{-1}=p=\operatorname{dim} \mathfrak{I}(\lambda)=\left[\mathcal{E}_{d}(\lambda)\right]
$$

Now put

$$
\Theta=\sum_{\omega \in \mathcal{\varepsilon}_{d}(\lambda)} d(\omega) \Theta_{\omega}-(-1)^{q} c^{-1} \sum_{s \in W(\lambda)} \varpi(s \lambda) \Theta_{s \lambda} .
$$

We have to show that $\Theta=0$. In view of Lemma 73, it would be enough to verify that $\Theta(f)=0$ for any $K$-finite function $f \in \mathcal{C}_{\rightarrow \lambda}(G)$. By the argument given above, we are reduced to the case when $f \in C_{\omega^{*}}(G)$ for some $\omega \in \mathcal{E}_{d}(\lambda)$. But then $\Theta(f)=0$ from Lemma 81 and Corollary 1 of Lemma 69. This completes the proof of Theorem 15. 


\section{\$ 41. Explicit determination of these characters}

Put $\varepsilon(\lambda)=\operatorname{sign} \varpi(\lambda)$ for $\lambda \in L^{\prime}$.

THEOREM 16. $\left({ }^{1}\right)$ For any $\lambda \in L^{\prime}$, there exists a unique element $\omega(\lambda) \in \mathcal{E}_{d}$ such that $\Theta_{\omega(\lambda)}=(-1)^{q} \varepsilon(\lambda) \Theta_{\lambda}$. The mapping $\lambda \rightarrow \omega(\lambda)$ of $L^{\prime}$ into $\mathcal{E}_{d}$ is surjective and

$$
d(\omega(\lambda))=c^{-1}\left[W_{G}\right]|\varpi(\lambda)|
$$

in the notation of Theorem 15. Finally $\omega\left(\lambda_{1}\right)=\omega\left(\lambda_{2}\right)\left(\lambda_{1}, \lambda_{2} \in L^{\prime}\right)$, if and only if $\lambda_{1}, \lambda_{2}$ are conjugate under $W_{G}$.

We begin by proving the surjectivity first. Fix $\omega \in \mathcal{E}_{d}$. Then by Lemma $78, \Phi_{\omega}$ is a finite linear combination of the characters of $B$. Introduce an order on $\mathfrak{F}$ and let $\lambda$ be the highest element in $L$ such that

$$
c_{0}=\int_{B} \Phi_{\omega} \operatorname{conj} \xi_{\lambda} d b \neq 0 .
$$

(As before, $\xi_{\lambda}$ has the same meaning as in $[4(\mathrm{p}), \S 24]$.) Then $\lambda \in L^{\prime}$.

$\operatorname{LgMMA} 82 . \Theta_{\omega}=(-1)^{q} \varepsilon(\lambda) \Theta_{\lambda}$.

For the proof of this lemma, we may, by going over to a finite covering group of $G$, assume that $K$ is also acceptable (see $[4(0), \S 18]$ ). Let $P$ be the set of all positive roots of $(\mathfrak{g}, \mathfrak{b})$ and $P_{0}, P_{+}$respectively the sets of all compact and singular roots in $P$ (see [4(n), § 4]). Put

$$
\varrho=\frac{1}{2} \sum_{\alpha \in P} \alpha, \quad \varrho_{0}=\frac{1}{2} \sum_{\alpha \in P_{0}} \alpha, \quad \varrho_{+}=\frac{1}{2} \sum_{\alpha \in P_{+}} \alpha
$$

Then $\varrho, \varrho_{0}, \varrho_{+}$are all in $L$ and $\varrho=\varrho_{0}+\varrho_{+}$. Hence we can define two analytic functions $\Delta_{0}$ and $\Delta_{+}$as follows.

$$
\Delta_{0}(\exp H)=\prod_{\alpha \in P_{0}}\left(e^{\alpha(H) / 2}-e^{-\alpha(H) / 2}\right), \quad \Delta_{+}(\exp H)=\prod_{\alpha \in P_{+}}\left(e^{\alpha(H) / 2}-e^{-\alpha(H) / 2}\right) \quad(H \in \mathfrak{b}),
$$

so that $\Delta=\Delta_{0} \Delta_{+}$. It is clear that

for $s \in W_{G}$.

$$
\Delta_{0}\left(b^{s}\right)=\varepsilon(s) \Delta_{0}(b), \Delta_{+}\left(b^{s}\right)=\Delta_{+}(b) \quad(b \in B)
$$

Let $d b$ and $d k$ denote the normalized Haar measures on $B$ and $K$ respectively. Fix a function $\alpha \in C_{c}{ }^{\infty}(G)$ such that $\alpha$ is invariant under right translations by $K$ and

$$
\int \alpha(x) d x=1
$$

(1) Cf. [4 (c), p. 40] and [4(d), Theorem 4]. 
For any $\beta \in C_{c}^{\infty}\left(B^{\prime}\right)$, we can define $f_{\beta} \in C_{c}^{\infty}\left(G_{B}\right)$ (see $[4(0), \S 20]$ ) by

$$
f_{\beta}\left(b^{x}\right)=\alpha(x) \Delta(b)^{-1} \sum_{s \in W_{G}} \varepsilon(s) \beta\left(b^{s}\right) \quad\left(x \in G, b \in B^{\prime}\right) .
$$

Similarly define $g_{\beta} \in C^{\infty}(K)$ by

$$
g_{\beta}\left(b^{k}\right)=\Delta_{+}(b) \Delta_{0}(b)^{-1} \sum_{s \in W_{a}} \varepsilon(s) \beta\left(b^{s}\right) \quad\left(k \in K, b \in B^{\prime}\right) .
$$

Fix $\pi \in \omega$ and let $U$ be the representation space of $\pi$. Define $E_{b}$ and $U_{\mathfrak{b}}\left(\triangleright \in \mathcal{E}_{R}\right)$ as usual and put

$$
\phi_{\mathrm{b}}\left(x_{\mathfrak{l}}\right)=\operatorname{tr}\left(E_{\mathrm{b}} \pi(x) E_{\mathrm{b}}\right) \quad(x \in G)
$$

Then $\phi_{\mathfrak{D}}(k)=n(\mathfrak{D}) \chi_{\mathfrak{D}}(k)(k \in K)$, where $n(\mathfrak{D})$ is a nonnegative integer and $\chi_{\mathfrak{D}}$ is the character of $\mathfrak{D}$. Moreover, as we have seen in $\S 25$, there exists an integer $N \geqslant 1$ such that $n(\delta) \leqslant N d(\delta)\left(\delta \in \mathcal{E}_{E}\right)$.

Put $m=\frac{1}{2} \operatorname{dim} G / B, m_{0}=\frac{1}{2} \operatorname{dim} K / B$ and

$$
T_{\beta}=\int_{K} g_{\beta}(k) \pi(k) d k, \quad \pi\left(f_{\beta}\right)=\int_{G} f_{\beta}(x) \pi(x) d x \quad\left(\beta \in C_{c}^{\infty}\left(B^{\prime}\right)\right) .
$$

Then $m=m_{0}+q$ and it is clear that

$$
\begin{aligned}
\pi\left(f_{\beta}\right) & =(-1)^{m}\left[W_{G}\right]^{-1} \int_{B} \Delta(b) \sum_{s \in W_{G}} \varepsilon(s) \beta\left(b^{s}\right) d b \int_{G} \alpha(x) \pi\left(b^{x}\right) d x \\
& =(-1)^{m} \int_{B} \Delta(b) \beta(b) d b \int_{G} \alpha(x) \pi\left(b^{x}\right) d x
\end{aligned}
$$

since $\alpha(x k)=\alpha(x) \quad(k \in K)$. Similarly

$$
T_{\beta}=(-1)^{m_{0}}\left[W_{G}\right]^{-1} \int_{B} \Delta_{0}(b)^{2} g_{\beta}(b) d b \int_{E} \pi\left(b^{k}\right) d k=(-1)^{m_{0}} \int_{B} \Delta(b) \beta(b) d b \int_{E} \pi\left(b^{k}\right) d k
$$

Therefore if follows that

$$
\pi\left(f_{\beta}\right)=(-1)^{q} \int_{G} \alpha(x) \pi(x) T_{\beta} \pi\left(x^{-1}\right) d x
$$

Now, by [4 (c), Lemma 24], the operator $T_{\beta}$ is summable and therefore it follows easily that

$$
\Theta_{\omega}\left(f_{\beta}\right)=\operatorname{tr} \pi\left(f_{\beta}\right)=(-1)^{q} \operatorname{tr} T_{\beta}=(-1)^{q} \sum_{\delta \in \mathcal{E}_{\boldsymbol{K}}} n(\delta) \int_{\boldsymbol{K}} g_{\beta}(k) \chi_{\delta}(k) d k
$$

On the other hand, $\quad \Theta_{\omega}\left(f_{\beta}\right)=\int \Theta_{\omega} f_{\beta} d x=(-1)^{m} \int_{B} \Phi_{\omega} \beta d b$.

7-662900. Acta mathematica. 116. Imprimé le 14 juin 1966. 
Therefore we have obtained the following result.

Lemma 83. $P$ ut $\eta_{\delta}(b)=\Delta_{0}(b) \chi_{\triangleright}(b)\left(\delta \in \mathcal{E}_{K}, b \in B\right)$. Then

$$
\int_{B} \Phi_{\omega} \beta d b=\sum_{\delta \in \varepsilon_{R}} n(\mathfrak{D}) \int_{B} \Delta_{+} \beta \eta_{\mathfrak{D}} d b
$$

for $\beta \in C_{c}^{\infty}\left(B^{\prime}\right)$, the series being absolutely convergent.

Define $\Re$ and $\Omega$ as in the proof of Lemma 5 and let $\bigcap_{K}$ denote the center of $\Re$. Then $\Omega \in B_{K}$. Let $u$ be the image of $\Omega$ in $S\left(\mathfrak{b}_{c}\right)$ under the canonical isomorphism (see [4 (e), Lemma 19]) of $Z_{K}$ into $\subseteq\left(\mathfrak{b}_{c}\right)$. Then it follows from [4(e), Theorem 2] that

$$
u \eta_{\mathrm{o}}=c(\delta) \eta_{\mathrm{o}} \quad\left(\delta \in \mathcal{E}_{E}\right)
$$

in the notation of Lemma 6 . Hence

$$
\int \beta \eta_{\triangleright} d b=c(\delta)^{-p} \int u^{* p} \beta \cdot \eta_{\triangleright} d b \quad\left(\beta \in C^{\infty}(B)\right),
$$

where $u^{*}$ is the adjoint of the differential operator $u$ and $p$ any positive integer. It follows from Weyl's formula for $\chi_{b}$ that $\left|\eta_{b}\right| \leqslant\left[W_{G}\right]$. Therefore

$$
\sum_{\mathfrak{D} \in \mathcal{\varepsilon}_{K}} n(\mathfrak{D})\left|\int \beta \eta_{\mathfrak{\delta}} d b\right| \leqslant N\left[W_{G}\right] \sup \left|u^{* p} \beta\right| \sum_{\mathfrak{D} \in \mathcal{E}_{K}} c(\mathfrak{D})^{-p} d(\mathfrak{D}),
$$

and so we conclude from Lemma 7 that there exists a distribution $S_{0}$ on $B$ such that

$$
S_{\mathbf{0}}(\beta)=\sum_{D \in \varepsilon_{B}} n(\delta) \int_{B} \beta \eta_{\triangleright} d b \quad\left(\beta \in C^{\infty}(B)\right)
$$

Put

$$
S=\Phi_{\omega}-\Delta_{+} S_{0}
$$

Then it follows from Lemma 83 that $S=0$ on $B^{\prime}$. Therefore since $B$ is compact, we can choose (see $[4(\mathrm{~m})$, Lemma $2 \mathrm{l}]$ ) an integer $p \geqslant 0$ such that $\Delta^{p} S=0$. This means that

$$
\int \Phi_{\omega} \Delta^{p} \beta d b=\sum_{\delta \in \mathcal{E}_{\boldsymbol{K}}} n(\mathfrak{D}) \int \beta \Delta^{p} \Delta_{+} \eta_{\triangleright} d b
$$

for $\beta \in C^{\infty}(B)$. Now put $\beta=\operatorname{conj} \xi_{\lambda+p e}$. Then it is clear that the left side is equal to $c_{0}$. Moreover, we know from Weyl's formula that $\Delta^{p} \Delta_{+} \eta_{\mathrm{o}}$ is a finite linear combination of characters of $B$ with coefficients in $\mathbf{Z}$. Therefore, since $n(\delta)$ is an integer, we conclude that $c_{0} \in \mathbf{Z}$. Since $c_{0} \neq 0$, this shows that $\left|c_{0}\right| \geqslant 1$. 
On the other hand, let $s_{1}=1, s_{2}, \ldots, s_{r}$ be a complete set of representatives in $W(\lambda)$ for $W_{G} \backslash W(\lambda)$. Then, by Lemma 78 there exist unique complex numbers $c_{\mathfrak{i}}$ such that

$$
\Theta_{\omega}=\sum_{1 \leqslant i \leqslant r} \varepsilon\left(s_{i}\right) c_{i} \Theta_{s_{i} \lambda}
$$

It is obvious that $c_{1}=c_{0}$ and we know from Corollary 2 of Lemma 81 that

$$
\sum_{1 \leqslant i \leqslant r}\left|c_{i}\right|^{2}=1
$$

Therefore $c_{1}=c_{0}= \pm 1$ and $c_{i}=0$ for $i \geqslant 2$. This shows that

$$
\Theta_{\omega}=c_{0} \Theta_{\lambda}
$$

By Theorem 15, there exist exactly $r$ distinct elements $\omega_{1}=\omega, \omega_{2}, \ldots, \omega_{r}$ in $\mathcal{E}_{d}(\lambda)$. For each $i$, we can, by the above proof, choose $s_{i} \in W(\lambda)\left(s_{1}=1\right)$ and a number $c_{i}= \pm 1$ such that

$$
\Theta_{\omega_{i}}=c_{i} \Theta_{s_{i} \lambda} \quad(1 \leqslant i \leqslant r)
$$

Then it follows from the linear independence of $\Theta_{\omega_{i}}$ (Lemma 81) that $s_{1}, s_{2}, \ldots, s_{r}$ form a complete system of representatives of $W_{G} \backslash W(\lambda)$. Therefore

$$
\sum_{i} d\left(\omega_{i}\right) \Theta_{\omega_{i}}=(-1)^{a} c^{-1}\left[W_{G}\right] \sum_{i} c_{i} \varpi\left(s_{i} \lambda\right) \Theta_{\omega_{i}}
$$

from Theorem 15 and this shows that

$$
d\left(\omega_{i}\right)=(-1)^{q} c^{-1}\left[W_{G}\right]|\varpi(\lambda)| c_{i} \varepsilon\left(s_{i} \lambda\right) \quad(1 \leqslant i \leqslant r) .
$$

But $d\left(\omega_{i}\right)>0$ and so we conclude that $c_{i}=(-1)^{\alpha} \varepsilon\left(s_{i} \lambda\right)$ and

$$
d\left(\omega_{i}\right)=c^{-1}\left[W_{G}\right]|\varpi(\lambda)|
$$

Hence in particular $c_{0}=c_{1}=(-1)^{q} \varepsilon(\lambda)$ and this proves Lemma 82 .

We now come to Theorem 16 . Since a class $\omega \in \mathcal{E}$ is completely determined by its character (see $\left[4(b)\right.$, p. 250]), the uniqueness of $\omega(\lambda)\left(\lambda \in L^{\prime}\right)$ is obvious. Moreover since $\Theta_{s \lambda}=$ $\varepsilon(s) \Theta_{\lambda}\left(s \in W_{G}\right)$ from the definition of $\Theta_{\lambda}$ (see [4 (p), Theorem 3]), it follows from the linear independence of the characters, that $\omega\left(\lambda_{1}\right)=\omega\left(\lambda_{2}\right)\left(\lambda_{1}, \lambda_{2} \in L^{\prime}\right)$ if and only if $\lambda_{1}, \lambda_{2}$ are conjugate under $W_{G}$. Now fix $\lambda \in L^{\prime}$ and let $r=\operatorname{dim} \mathfrak{T}(\lambda)$. Then by Theorem 15 , there are exactly $r$ distinct elements $\omega_{1}, \omega_{2}, \ldots, \omega_{r}$ in $\mathcal{E}_{d}(\lambda)$. Moreover, from the above proof, we can choose a complete set of representatives $\left(s_{1}, s_{2}, \ldots, s_{r}\right)$ for $W_{G} \backslash W(\lambda)$ such that

$$
\Theta_{\omega_{i}}=(-1)^{q} \varepsilon\left(s_{i} \lambda\right) \Theta_{s_{i} \lambda} \quad(1 \leqslant i \leqslant r) .
$$


We may assume that $s_{1} \in W_{G}$ and therefore

$$
\Theta_{\omega_{1}}=(-1)^{a} \varepsilon\left(s_{1} \lambda\right) \Theta_{s_{1} \lambda}=(-1)^{q} \varepsilon(\lambda) \Theta_{\lambda}
$$

This shows that $\omega_{1}=\omega(\lambda)$. We have already seen that $d\left(\omega_{1}\right)=c^{-1}\left[W_{G}\right]|\omega(\lambda)|$ and therefore the proof of Theorem 16 is now complete.

Theorem 16 shows that

$$
c^{-1}(-1)^{q} \sum_{\lambda \in L} \varpi(\lambda) \Theta_{\lambda}=\sum_{\infty \in \varepsilon_{d}} d(\omega) \Theta_{\omega}
$$

and we know (see $[4(\mathrm{~d}), \S 5]$ ) that this distribution represents the contribution of the discrete series to the Plancherel formula of $G$.

\section{Part IV. Some inequalities and their consequences}

\section{\$ 42. Proof of the inequalities}

Let us use the notation of $\S 14$ and put $\mathfrak{a}_{2}=\mathfrak{h} \cap \mathfrak{p}, \mathfrak{n}_{2}=\mathfrak{n}_{\mathfrak{h}}, \mathfrak{a}_{1}=\mathfrak{a} \cap[\mathfrak{m}, \mathfrak{m}], \mathfrak{l}_{1}=\mathfrak{l} \cap \mathfrak{m}$, $\mathfrak{n}_{1}=\mathfrak{n} \cap \mathfrak{m}$ and $\mathfrak{m}_{1}=\mathfrak{l}_{1}+\mathfrak{a}_{1}+\mathfrak{n}_{1}$. We denote the analytic subgroup of $G$ corresponding to a subalgebra of $g$ by the corresponding capital latin letter e.g. $A_{2}$ and $N$ correspond to $\mathfrak{a}_{2}$ and $\mathfrak{n}$ respectively. Then $G=K A N$ and $M_{1}=K_{1} A_{1} N_{1}$ are the Iwasawa decompositions of $\mathrm{G}$ and $M_{1}$ respectively. For any $x \in G$, let $\varkappa(x)$ and $H(x)$ denote the unique elements $k \in K$ and $H \in \mathfrak{a}$ respectively, such that $x=k \exp H \cdot n(n \in N)$. Let $H_{i}(x)$ denote the component of $H(x)$ in $\mathfrak{a}_{i}(i=1,2)$ so that $H(x)=H_{1}(x)+H_{2}(x)$.

We fix orders in the duals of the real vector spaces $\mathfrak{a}_{2}, \mathfrak{a}$ and $\mathfrak{h}^{*}=\mathfrak{h} \cap \mathfrak{p}+(-1)^{\frac{1}{2}} \mathfrak{h} \cap \mathfrak{l}$ and assume that they are compatible for the pairs $\left(\mathfrak{a}, \mathfrak{a}_{2}\right)$ and $\left(\mathfrak{h}^{*}, \mathfrak{a}_{2}\right)$. Let $P$ denote the set of positive roots of $(\mathfrak{g}, \mathfrak{h})$ and $\Sigma$ the set of positive roots of $(\mathfrak{g}, \mathfrak{a})$. Let $P_{1}$ and $\Sigma_{1}$ be the sets of those elements in $P$ and $\Sigma$ respectively, whose restrictions on $\mathfrak{a}_{2}$ are zero. We denote by $P_{2}$ and $\Sigma_{2}$ the complements of $P_{1}$ and $\Sigma_{1}$ in $P$ and $\Sigma$ respectively.

Put $M_{1 \mathfrak{p}}=\exp \left(\mathrm{m}_{1} \cap \mathfrak{p}\right)$ so that $G=K M_{1 \mathfrak{p}} A_{2} N_{2}$ (see [4 (g), Lemma 11]). Fix $x \in G$ and let $x=k \operatorname{man}\left(k \in K, m \in M_{1 p}, a \in A_{2}, n \in N_{2}\right)$. Then $k, m, a, n$ are uniquely determined. Put $\mu(x)=m$. Since $M_{1}$ and $A_{2}$ commute, it is easy to verify that

$$
\varkappa(x)=k x(m), \quad H_{2}(x)=\log a, \quad H_{1}(x)=H(m) .
$$

Define $\varrho$ and $\Xi_{1}=\Xi_{M}$ as in $\S 14$. Since $\mathfrak{m}$ normalizes $\mathfrak{n}_{2}$, it is clear that $\operatorname{tr}(\operatorname{ad} X)_{\mathfrak{H}_{2}}=0$ for $X \in[\mathfrak{m}, \mathfrak{m}]$. Therefore since $\mathfrak{n}=\mathfrak{n}_{1}+\mathfrak{n}_{2}$, we conclude that

$$
\varrho(H)=\frac{1}{2} \operatorname{tr}(a d H)_{\mathfrak{n}_{1}} \quad\left(H \in \mathfrak{a}_{1}\right) .
$$


Lемма 84. Let $d_{1} k$ denote the normalized Haar measure on $K_{1}$. Then

$$
\int_{K_{1}} e^{-\varrho(H(x k))} d_{1} k=e^{-\varrho\left(H_{2}(x)\right)} \Xi_{1}(\mu(x)) \quad(x \in G)
$$

Since $K_{1}$ normalizes $N_{2}$, it is easy to see that

$$
H(x k)=H_{1}(x k)+H_{2}(x k)=H(\mu(x k))+H_{2}(x)=H(\mu(x) k)+H_{2}(x) \quad\left(k \in K_{1}\right) .
$$

Hence our assertion follows from the fact (see [4 (q), Lemma 31]) that

$$
\Xi_{1}(m)=\int_{K_{1}} e^{-\varrho(H(m k))} d_{1} k \quad\left(m \in M_{1}\right)
$$

As usual let $d k$ denote the normalized Haar measure on $K$. Then the following result is an immediate consequence of Lemma 84 and [4 (q), Lemma 31].

Corollary.

$$
\int_{K} e^{-\varrho\left(H_{2}(x k)\right)} \Xi_{1}(\mu(x k)) d k=\Xi(x) \quad(x \in G)
$$

Put $\vec{N}=\theta(N), \bar{N}_{i}=\theta\left(N_{i}\right)(i=1,2)$ and

$$
\beta(H)=\inf _{\alpha \in \Sigma_{\mathbf{z}}} \alpha(H) \quad(H \in \mathfrak{a})
$$

Lемма 85. $\varrho\left(H_{2}(\bar{n})\right) \geqslant 0$ and $\varrho(H(\bar{n})) \geqslant 0$ for $\bar{n} \in \bar{N}$. Moreover, if $a \in A_{2}$ and $\beta(\log a) \geqslant 0$, we have

and

$$
\exp \varrho\left(H_{2}\left(\bar{n}^{a}\right)\right) \leqslant 1+\exp \left\{-\frac{1}{2} \beta(\log a)+\varrho\left(H_{2}(\bar{n})\right)\right\} \quad(\bar{n} \in \bar{N})
$$$$
\exp \varrho\left(H\left(\bar{n}^{a}\right)\right) \leqslant 1+\exp \{-\beta(\log a)+\varrho(H(\bar{n}))\} \quad\left(\bar{n} \in \bar{N}_{2}\right) \text {. }
$$

Let $Z$ be the center of $G$. Then, for the purpose of this lemma, we can obviously replace $G$ by $G / Z$. Hence we may agree to subscribe to the assumptions and conventions of $[4(\mathrm{j})$, p. 244].

For any linear function $\lambda$ on $\mathfrak{h}_{c}$, define $H_{\lambda} \in \mathfrak{h}_{c}$ as usual by the condition

$$
\operatorname{tr}\left(\operatorname{ad} H \operatorname{ad} H_{\lambda}\right)=\lambda(H) \quad\left(H \in \mathfrak{h}_{c}\right) .
$$

Also put $\left\langle\lambda_{1}, \lambda_{2}\right\rangle=\lambda_{1}\left(H_{\lambda_{2}}\right)$ for two such functions $\lambda_{1}, \lambda_{2}$. Let $J_{P}$ denote the set of all $\lambda$ such that $2\langle\lambda, \alpha\rangle\left\langle\langle\alpha, \alpha\rangle\right.$ is a nonnegative integer for every $\alpha \in P$. Then for every $\lambda \in J_{P}$, we have an irreducible representation $\pi_{\lambda}$ of $G$ on a finite-dimensional (complex) Hilbert space $V_{\lambda}$ with the highest weight $\lambda$ (with respect to $\mathfrak{h}$ ). We denote the corresponding representation of $\mathbb{B S}$ also by $\pi_{\lambda}$. Let $v_{\lambda}$ denote a unit vector in $V_{\lambda}$ belonging to the highest weight $\lambda$. 
Lемма 86. Fix $\lambda \in J_{P}$ and let $U$ be the subspace consisting of all $v \in V_{\lambda}$ such that $\pi_{\lambda}(H) v=\lambda(H) v$ for all $H \in \mathfrak{a}_{2}$. Then $U$ is invariant and irreducible under $\pi_{\lambda}(\mathrm{m})$. Moreover, $\operatorname{dim} U=1$ if and only if $\langle\lambda, \alpha\rangle=0$ for $\alpha \in P_{1}$.

We write $V$ and $\pi$ for $V_{\lambda}$ and $\pi_{\lambda}$ respectively. It is clear that

$$
\mathfrak{g}=\theta\left(\mathfrak{n}_{2}\right)+\mathfrak{m}+\mathfrak{n}_{2}
$$

and therefore

$$
\mathfrak{G}=\theta\left(\mathfrak{N}_{2}\right) \mathfrak{M N}_{2} \text {, }
$$

where $\left(^{1}\right) \mathfrak{M}=\subseteq\left(\mathfrak{m}_{c}\right)$ and $\mathfrak{R}_{2}=\subseteq\left(\left(\mathfrak{n}_{2}\right)_{c}\right)$.

For any $\alpha \in P$ define $X_{\alpha}, X_{-\alpha}$ as in [4 (n), § 4] and put

$$
\mathfrak{l}_{\alpha}=\mathbf{C} H_{\alpha}+\mathbf{C X} X_{\alpha}+\mathbf{C X} X_{-\alpha} .
$$

Fix $u \neq 0$ in $U$. Then if $\alpha \in P_{2}$, it is clear that

$$
\pi\left(H X_{\alpha}\right) u=(\lambda(H)+\alpha(H)) \pi\left(X_{\alpha}\right) u \quad\left(H \in \mathfrak{a}_{2}\right) .
$$

Since $\lambda$ is the highest weight of $\pi$, we conclude from the definition of our order, that $\pi\left(X_{\alpha}\right) u=0$. Hence

$$
V=\pi(\mathfrak{S}) u=\pi\left(\theta\left(\mathfrak{N}_{2}\right) \mathfrak{M}\right) u
$$

But then it is obvious from the definition of $U$ that $U=\pi(\mathfrak{M}) u$. This proves that $U$ is invariant and irreducible under $\pi(\mathrm{m})$.

Now fix $\alpha \in P$ and observe that $\pi\left(X_{\alpha}\right) v_{\lambda}=0$. Hence by considering the subalgebra $\mathfrak{l}_{\alpha}$, it follows (see [4(m), Lemma 25]) that $\pi\left(X_{-\alpha}\right) v_{\lambda}=0$ if and only if $\langle\lambda, \alpha\rangle=0$. On the other hand, $\mathfrak{m}^{\prime}=[\mathfrak{m}, \mathfrak{m}]$ is clearly generated, as a Lie algebra, by $\theta\left(\mathfrak{n}_{1}\right)+\mathfrak{n}_{1}$. Hence $\pi\left(\mathfrak{m}^{\prime}\right) v_{\lambda}=$ $\{0\}$ if and only if $\langle\lambda, \alpha\rangle=0$ for all $\alpha \in P_{1}$. Since $U$ is irreducible under $\pi(\mathfrak{m})$, the second assertion of the lemma is now obvious

LEMмA 87. Fix $\Lambda \in J_{P}$. Then $\Lambda\left(H_{2}(\bar{n})\right) \geqslant 0$ and

$$
\exp \Lambda\left(H_{2}\left(\bar{n}^{a}\right)\right) \leqslant 1+\exp \left\{-\frac{1}{2} \beta(\log a)+\Lambda\left(H_{2}(\bar{n})\right)\right\}
$$

for $\bar{n} \in \bar{N}$ and $a \in A_{2}$ provided $\beta(\log a) \geqslant 0$.

Put $\lambda=\Lambda-\theta \Lambda$ so that

$$
\langle\lambda, \alpha\rangle=\langle\Lambda, \alpha\rangle-\langle\Lambda, \theta \alpha\rangle \quad(\alpha \in P)
$$

Obviously this is zero if $\alpha \in P_{1}$. On the other hand, $-\theta \alpha \in P_{2}$ whenever $\alpha \in P_{2}$ and therefore,

(1) We use here the notation of [4 (m), p. 280]. 
since (1) $|\theta \alpha|^{2}=|\alpha|^{2}$, it follows that $\lambda \in J_{P}$. Put $\pi=\pi_{\lambda}, V=V_{\lambda}, v=v_{\lambda}$ and observe that $\lambda=2 \Lambda$ on $\mathfrak{h} \cap \mathfrak{p}=\mathfrak{a}_{2}$ and $\lambda=0$ on $\mathfrak{h} \cap \mathfrak{f}$. Hence $\operatorname{dim} U=1$ and $\pi\left(\mathfrak{m}^{\prime}\right) U=\{0\}$ from Lemma 86 . On the other hand, it is easy to see that $m_{1} \cap \mathfrak{p} \subset \mathfrak{m}^{\prime}$ so that $\pi(m) v=v$ for $m \in M_{1 \mathfrak{p}}$. Therefore it is obvious that

$$
|\pi(x) v|=e^{\lambda\left(H_{2}(x)\right)}=e^{2 \Lambda\left(H_{2}(x)\right)} \quad(x \in G) .
$$

Let $E$ denote the orthogonal projection of $V$ on $U$. Then if $X \in \theta\left(\mathfrak{n}_{2}\right)$, it is obvious that $E \pi\left(X^{r}\right) v=0$ for $r \geqslant 1$. Moreover, $\theta\left(\mathfrak{n}_{1}\right) \subset \mathfrak{m}^{\prime}$ and therefore $\pi\left(\theta\left(\mathfrak{n}_{1}\right)\right) v=\{0\}$. On the other hand, $\mathbb{n}_{2}$ is an ideal in $\mathfrak{n}$ and therefore $\bar{N}=\bar{N}_{2} \bar{N}_{1}$. Hence $E \pi(\bar{n}) v=v$ and this shows that $|\pi(\bar{n}) v| \geqslant|v|=1(\bar{n} \in \bar{N})$. Hence

$$
\Lambda\left(H_{2}(\bar{n})\right) \geqslant 0 \quad(\bar{n} \in \bar{N}) .
$$

Put $E^{\prime}=1-E$ and let $\|T\|$ denote the Hilbert-Schmidt norm of a linear transformation $T$ in $V$. Since $U=\mathbf{C} v$, it is clear that

$$
\exp 4 \Lambda\left(H_{2}\left(\bar{n}^{a}\right)\right)=\left\|\pi\left(\bar{n}^{a}\right) E\right\|^{2}=\left\|E \pi\left(\bar{n}^{a}\right) E\right\|^{2}+\left\|E^{\prime} \pi\left(\bar{n}^{a}\right) E\right\|^{2}=1+\left\|E^{\prime} \pi\left(\bar{n}^{a}\right) E\right\|^{2}
$$

since $E \pi\left(\bar{n}^{a}\right) E=E$ as we saw above. On the other hand, we have seen during the proof of Lemma 86 that

$$
V=\pi\left(\theta\left(\mathfrak{R}_{2}\right) \mathfrak{M}\right) v=\pi\left(\theta\left(\mathfrak{\Re}_{2}\right)\right) v
$$

Therefore every weight of $\pi$, other than $\lambda$, is of the form $\lambda-\sigma$ with

$$
\sigma=\sum_{1 \leqslant i \leqslant r} \alpha_{i}
$$

$\alpha_{i} \in P_{2}$ and $r \geqslant 1$. Let $\lambda=\lambda_{0}>\lambda_{1}>\ldots \lambda_{p}$ be all the weights of $\pi$ and $V_{i}$ the subspace of $V$ consisting of all vectors belonging to the weight $\lambda_{i}(0 \leqslant i \leqslant p)$. Since $\mathfrak{h}=\theta(\mathfrak{h}), V$ is the orthogonal sum of $V_{i}(0 \leqslant i \leqslant p)$. Put $\sigma_{i}=\lambda-\lambda_{i}$ and let $E_{i}$ denote the orthogonal projection of $V$ on $V_{i}$. Then it is clear that $E^{\prime}=E_{1}+\ldots+E_{p}$ and therefore

$$
E^{\prime} \pi\left(\bar{n}^{a}\right) E=\sum_{1 \leqslant i \leqslant p} e^{-\sigma_{i}(\log a)} E_{i} \pi(\bar{n}) E .
$$

On the other hand, since $\beta(\log a) \geqslant 0$, it is obvious that

Therefore

$$
\sigma_{i}(\log a) \geqslant \inf _{\alpha \in P_{2}} \alpha(\log a)=\beta(\log a) .
$$

$$
\begin{aligned}
\left\|E^{\prime} \pi\left(\bar{n}^{a}\right) E\right\|^{2} & \leqslant e^{-2 \beta(\log a)} \sum_{1 \leqslant i \leqslant p}\left\|E_{i} \pi(\bar{n}) E\right\|^{2} \\
& \leqslant e^{-2 \beta(\log a)}|\pi(\bar{n}) v|^{2}=\exp \left\{-2 \beta(\log a)+4 \Lambda\left(H_{2}(\bar{n})\right)\right\},
\end{aligned}
$$

and the assertion of the lemma is now obvious.

(1) As usual, $|\alpha|^{2}=\langle\alpha, \alpha\rangle$. 
Let $\pi$ be an irreducible finite-dimensional representation of $G$ on $V$ and $\Lambda$ the highest weight of $\pi$ with respect to a. Let $U$ be the subspace of those vectors $u \in V$ for which $\pi(H) u=\Lambda(H) u\left(H \in \mathfrak{a}_{2}\right)$. We denote by $E$ the orthogonal projection of $V$ on $U$.

Lемм A 88. Fix $a \in A_{2}$ such that $\beta(\log a) \geqslant 0$. Then

$$
\exp \Lambda\left(H\left(\bar{n}^{a}\right)\right) \leqslant 1+\exp \{-\beta(\log a)+\Lambda(H(\bar{n}))\}
$$

and

$$
\left\|\pi\left(\bar{n}^{a}\right) E\right\| \leqslant\|E\|+e^{-\beta(\log a)}\|\pi(\bar{n}) E\|
$$

for $\bar{n} \in \bar{N}_{2}$. Moreover, $\Lambda(H(\bar{n})) \geqslant 0$ for $\bar{n} \in \bar{N}$.

Let $\Lambda=\Lambda_{1}>\Lambda_{2}>\ldots>\Lambda_{p}$ be all the weights of $\pi$ with respect to a. It follows from the definition of our orders that there exists an integer $q \geqslant 1$ such that $\sigma_{i}=\Lambda-\Lambda_{i}$ is zero on $\mathfrak{a}_{2}$ for $i \leqslant q$ while $\sigma_{i} \neq 0$ on $\mathfrak{a}_{2}$ for $i>q$. Let $E_{i}$ denote the orthogonal projection of $V$ on the space $V_{i}$ consisting of all vectors belonging to the weight $\Lambda_{i}$. Then $E=E_{1}+\ldots+E_{q}$ and

$$
E^{\prime}=1-E=\sum_{a<i \leqslant p} E_{i}
$$

Fix $i>q$. Then it is clear (see the proof of Lemma 86) that

$$
\sigma_{i}=\sum_{\alpha \in \Sigma} r(\alpha) \alpha
$$

where $r(\alpha)$ are nonnegative integers and $r(\alpha) \geqslant 1$ for some $\alpha \in \Sigma_{2}$. Hence if $v$ is a unit vector in $V_{1}$, it is clear that

$$
\left|\pi\left(\bar{n}^{a}\right) v\right|^{2}=1+\left|E^{\prime} \pi\left(\bar{n}^{\alpha}\right) v\right|^{2} \leqslant 1+e^{-2 \beta(\log a)}|\pi(\bar{n}) v|^{2} \quad\left(\bar{n} \in \bar{N}_{2}\right)
$$

and from this the first inequality follows immediately. Moreover,

$$
\begin{aligned}
\left\|\pi\left(\bar{n}^{a}\right) E\right\|^{2}=\|E\|^{2}+\left\|E^{\prime} \pi\left(\bar{n}^{a}\right) E\right\|^{2} & \leqslant\|E\|^{2}+e^{-2 \beta(\log a)}\left\|E^{\prime} \pi(\bar{n}) E\right\|^{2} \\
& \leqslant\|E\|^{2}+e^{-2 \beta(\log a)}\|\pi(\bar{n}) E\|^{2} \quad\left(\bar{n} \in \bar{N}_{2}\right)
\end{aligned}
$$

and this gives the second inequality. The last statement of Lemma 88 has already been proved in [4 (j), Lemma 43].

Now if we take $\Lambda=\frac{1}{2} \Sigma_{\alpha \in P} \alpha$ in Lemma 87, and choose $\pi$, in Lemma 88, such that its highest weight, with respect to $\mathfrak{a}$, is $\varrho$, then we get Lemma 85 immediately. $\left(^{1}\right)$

(1) The second inequality of Lemma 88 has been proved for later applications. 


\section{\$ 43. Applications of the above inequalities}

Define the number $d \geqslant 0$ as in $\S 14$ and observe that $\varrho(H(\bar{n})) \geqslant 0$ for $\bar{n} \in \bar{N}$ from Lemma 85. Let $d_{i} \bar{n}$ denote the Haar measure on $\bar{N}_{i}(i=1,2)$.

LeMMA 89. $\left.{ }^{1}\right)$ For any $\varepsilon>0$,

$$
\int_{\bar{N}_{3}} e^{-\varrho(H(\bar{n}))}\{1+\varrho(H(\bar{n}))\}^{-(d+\varepsilon)} d_{2} \bar{n} \leqslant \infty .
$$

We can choose $c>0$ (see [4 (j), Theorem 3]) such that

$$
e^{\varrho(\log a)} \Xi(a) \leqslant c(1+\sigma(a))^{d} \quad(a \in A) .
$$

On the other hand, by [4 (j), Cor. 2, p. 289],

$$
e^{\varrho(\log a)} \Xi(a)=\int_{\bar{N}} \exp \left\{-\varrho\left(H\left(\bar{n}^{a}\right)\right)-\varrho(H(\bar{n}))\right\} d \bar{n} \quad(a \in A),
$$

where $d \tilde{n}$ is the (suitably normalized) Haar measure on $\bar{N}$. We may assume that $d \bar{n}=$ $d_{2} \bar{n}_{2} \cdot d_{1} \bar{n}_{1}$ for $\bar{n}=\bar{n}_{2} \bar{n}_{1}\left(\bar{n}_{i} \in \bar{N}_{i}, i=1,2\right)$. Now

$$
\bar{n}=\bar{n}_{2} \bar{n}_{1} \in \bar{n}_{2} k^{-1} \exp H\left(\bar{n}_{1}\right) \cdot N_{1},
$$

where $k=\varkappa\left(\bar{n}_{1}\right)^{-1} \in K_{1}$. Since $M$ normalizes $\theta\left(\mathfrak{n}_{2}\right)$, we get

$$
H(\bar{n})=H\left(\bar{n}_{2}^{k}\right)+H\left(\bar{n}_{1}\right)
$$

We may normalize $d_{1} \bar{n}_{1}$ in such a way (see [4 (j), Lemma 44]) that

$$
\int_{\bar{N}_{2}} e^{-2 \varrho\left(H\left(\bar{n}_{1}\right)\right)} d_{1} \bar{n}_{1}=1
$$

Then, since $d_{2} \bar{n}_{2}^{k} / d_{2} \bar{n}_{2}=1\left(k \in K_{1}\right)$ and $A_{2}$ commutes with $\bar{N}_{1}$, we conclude that

$$
e^{\varrho(\log a)} \Xi(a)=\int_{\bar{N}_{2}} \exp \left\{-\varrho\left(H\left(\bar{n}^{a}\right)\right)-\varrho(H(\bar{n}))\right\} d_{2} \bar{n} \leqslant c(\mathbf{1}+\sigma(a))^{d} \quad\left(a \in A_{2}\right) .
$$

Put $a_{t}=\exp t H(t \in \mathbf{R})$ where $H$ is an element in $\mathfrak{a}_{2}$ such that

$$
b=\beta(H)>0 .
$$

Then if $a=a_{t}(t \geqslant 0)$, it follows from Lemma 85 that

and therefore

$$
\exp \varrho\left(H\left(\bar{n}^{a}\right)\right) \leqslant 1+\exp \{\varrho(H(\bar{n}))-b t\} \quad\left(\bar{n} \in \bar{N}_{2}\right)
$$

(i) Cf. [4 (j), Lemma 45]. 


$$
\int_{\bar{N}_{2}} e^{-\varrho(H(\bar{n}))}\left\{1+e^{\varrho(H(\bar{n}))-b t}\right\}^{-1} d_{2} \bar{n} \leqslant c\left(1+\sigma\left(a_{t}\right)\right)^{d} \leqslant c^{\prime}(1+t)^{d}
$$

for $t \geqslant 0$. Here $c^{\prime}$ is a positive constant independent of $t$. Let $\bar{N}_{2, r}$ denote the set of all $\bar{n} \in \bar{N}_{2}$ with $\varrho(H(\bar{n})) \leqslant 2^{r}$ and put $t=2^{r} b^{-1}$. Then

for $\bar{n} \in \bar{N}_{2, r}$ and therefore

$$
e^{\varrho(H(\bar{n}))-b t} \leqslant 1
$$

$$
\int_{\bar{N}_{2, r}} e^{-\varrho(H(\bar{n}))} d_{2} \bar{n} \leqslant 2 c^{\prime}\left(1+b^{-1} 2^{r}\right)^{d} \leqslant c_{1} 2^{r d} \quad(r \geqslant 0),
$$

where $c_{1}$ is a positive number independent of $r$. Let $\bar{N}_{2}(r)$ denote the complement of $\bar{N}_{2, r-1}$ in $\bar{N}_{2, r}(r \geqslant 1)$. Then

$$
\int_{\bar{N}_{2}(r)} e^{-\varrho(H(\bar{n}))}\{1+\varrho(H(\bar{n}))\}^{-(d+\varepsilon)} d_{2} \bar{n} \leqslant c_{1} 2^{r d-(r-1)(d+\varepsilon)}=c_{1} 2^{d-(r-1) \varepsilon} \quad(r \geqslant 1) .
$$

Since $\bar{N}_{2.0}$ is compact [4 (j), Lemma 40], we get the required result from the convergence of the series $\sum_{r \geqslant 0} 2^{-r \varepsilon}$.

LEMмA 90. Put $\bar{n}=\theta\left(n^{-1}\right)$ for $n \in N$. Then there exists a number $c \geqslant 1$ such that

and

$$
1+\max (\sigma(h), \varrho(H(\bar{n}))) \leqslant c(1+\sigma(h n))
$$

$$
\Xi(h n) \leqslant c(1+\sigma(h n))^{d} \exp \{-\varrho(\log h)-\varrho(H(\bar{n}))\}
$$

for $h \in A$ and $n \in N$.

It is clear that, for the proof of this lemma, we may assume, as in $\S 42$, that the conditions of $[4(\mathrm{j})$, p. 244] hold. Fix an irreducible finite-dimensional representation $\pi$ of $G$ with the highest weight $\varrho$ with respect to $\mathfrak{a}$. Define $A^{+}$as in $\S 14$. Then $G=K A^{+} K$ and therefore $h n=k_{1} h^{\prime} k_{2}\left(k_{1}, k_{2} \in K ; h^{\prime} \in A^{+}\right)$. Then

$$
\bar{n} h=\theta(h n)^{-1}=k_{2}^{-1} h^{\prime} k_{1}^{-1}
$$

and therefore $\|\pi(h n)\|=\left\|\pi\left(h^{\prime}\right)\right\|=\|\pi(\bar{n} h)\|$. Hence if $p$ is the degree of $\pi$, we get

$$
e^{2 \varrho\left(\log h^{\prime}\right)} \leqslant\left\|\pi\left(h^{\prime}\right)\right\|^{2} \leqslant p e^{2 \varrho\left(\log h^{\prime}\right)} .
$$

Let $\mathfrak{w}$ be the Weyl group of $\mathfrak{g}$ with respect to $\mathfrak{a}$ (see [4 (j), p. 249]). Fix $s \in \mathfrak{w}$ and choose $k \in K$ such that $\operatorname{Ad}(k) H=s H$ for all $H \in \mathfrak{a}$. Then (see $[1, \S 7.4]) \bar{n}^{k}=\bar{n}_{1} n_{2}$ where

$$
\bar{n}_{1} \in \bar{N} \cap \bar{N}^{k}, \quad n_{2} \in N \cap \bar{N}^{k} .
$$


Hence

$$
\| \pi\left(h^{\prime}\|=\| \pi\left(\bar{n}^{k} h^{s}\right)\|=\| \pi\left(\bar{n}_{1} h^{s} n_{2}^{\prime}\right) \|,\right.
$$

where $n_{2}^{\prime}=\left(h^{s}\right)^{-1} n_{2} h^{s}$. Therefore

$$
\left\|\pi\left(h^{\prime}\right)\right\|^{2} \geqslant\left|\pi\left(\tilde{n}_{1} h^{s}\right) \psi\right|^{2} \geqslant e^{2 \varrho\left(\log h^{s}\right)}
$$

where $\psi$ is a unit vector belonging to the highest weight $\varrho$. This shows that

$$
\varrho\left(\log h^{\prime}\right)+\frac{1}{2} \log p \geqslant \max _{s \in \mathfrak{w}} \varrho\left(\log h^{s}\right) \geqslant|\varrho(\log h)|
$$

(see [4 (j), p. 281]). On the other hand, we can obviously choose $c_{1}, c_{2}>0$ such that

$$
c_{1} \sigma\left(h_{1}\right) \leqslant \varrho\left(\log h_{1}\right) \leqslant c_{2} \sigma\left(h_{1}\right) \quad\left(h_{1} \in A^{+}\right) .
$$

Then

$$
c_{2} \sigma\left(h^{\prime}\right)+\frac{1}{2} \log p \geqslant \max _{s \in \mathfrak{w}} \varrho\left(\log h^{s}\right) \geqslant c_{1} \sigma(h) .
$$

Since $\sigma\left(h^{\prime}\right)=\sigma(h n)$, this shows that we can choose $c_{3}>0$ such that

$$
c_{3}(1+\sigma(h n)) \geqslant 1+\sigma(h) \quad(h \in A, n \in N) .
$$

Moreover, we know [4 (k), Lemma 42] that

$$
\varrho\left(\log h^{\prime}\right) \geqslant \varrho(\log h)+\varrho(H(\bar{n})) .
$$

Since

$$
\varrho\left(\log h^{\prime}\right)+\frac{1}{2} \log p \geqslant-\varrho(\log h)
$$

by our result above, we conclude that

$$
2 \varrho\left(\log h^{\prime}\right)+\frac{1}{2} \log p \geqslant \varrho(H(\bar{n})) .
$$

Hence we can choose $c_{4}>0$ such that

$$
1+\varrho(H(\bar{n})) \leqslant c_{4}(1+\sigma(h n)) \quad(h \in A, n \in N) .
$$

Now select $c_{5}>0$ such that

Then

$$
\Xi\left(h_{1}\right) \leqslant c_{5} e^{-\varrho\left(\log h_{1}\right)}\left(1+\sigma\left(h_{1}\right)\right)^{d} \quad\left(h_{1} \in A^{+}\right) .
$$

$\Xi(h n)=\Xi\left(h^{\prime}\right) \leqslant c_{5} e^{-\varrho\left(\log h^{\prime}\right)}\left(1+\sigma\left(h^{\prime}\right)\right)^{d} \leqslant c_{5}(1+\sigma(h n))^{d} \exp \{-\varrho(\log h)-\varrho(H(\bar{n}))\}$.

This proves Lemma 90.

Put $\varrho_{i}(H)=\operatorname{tr}(\operatorname{ad} H)_{\mathfrak{n}_{i}}(H \in \mathfrak{a}, i=1,2)$ so that $\varrho=\varrho_{1}+\varrho_{2}$. 
COROLLARY 1. Suppose $r_{1}, r_{2}$ are two numbers $\geqslant 0$ and $r=r_{1}+r_{2}$. Then

$e^{\varrho_{2}(\log h)} \Xi(h n)(1+\sigma(h n))^{-(r+d)} \leqslant c^{r+1} e^{-\varrho_{1}(\log h)}(1+\sigma(h))^{-r_{1}} e^{-\varrho(H(\bar{n}))}(1+\varrho(H(\bar{n})))^{-r_{z}}$

for $h \in A$ and $n \in N$.

For, in the above notation, we have

$$
\Xi(h n)(1+\sigma(h n))^{-(r+d)}=\Xi\left(h^{\prime}\right)\left(1+\sigma\left(h^{\prime}\right)\right)^{-(r+d)} \leqslant c e^{-\underline{g\left(\log h^{\prime}\right)}}\left(\mathbf{1}+\sigma\left(h^{\prime}\right)\right)^{-\gamma} .
$$

But

$$
\left(1+\sigma\left(h^{\prime}\right)\right)^{-r} \leqslant c^{r}(1+\sigma(h))^{-r_{1}}(1+\varrho(H(\bar{n})))^{-r_{2}}
$$

and

$$
\varrho\left(\log h^{\prime}\right) \geqslant \varrho(\log h)+\varrho(H(\bar{n})) .
$$

Hence our assertion is obvious.

Let $d_{2} n$ denote the Haar measure on $N_{2}$.

COROLLARY 2. Let $\Omega$ be a compact set in $G$. Then if $r>2 d$, the integral

converges uniformly for $x \in \Omega$.

$$
\int_{N_{3}} \Xi(x n)(1+\sigma(x n))^{-r} d_{2} n
$$

Let $x=k_{0} h n_{0}\left(k_{0} \in K, h \in A, n_{0} \in N\right)$. Then $\Xi(x n)=\Xi\left(h n_{0} n\right)$ and $\sigma(x n)=\sigma\left(h n_{0} n\right)$. Now let $\bar{n}_{0}=\bar{n}_{2} \bar{n}_{1}$ where $\bar{n}_{i} \in \bar{N}_{i}(i=1,2)$. Then $h$ and $\bar{n}_{i}$ remain bounded $\left(^{1}\right)$ and

$$
H\left(\theta\left(n_{0} n\right)^{-1}\right)=H\left(\bar{n} \bar{n}_{2} \bar{n}_{1}\right)=H\left(\bar{n} \bar{n}_{2} k^{-1}\right)+H\left(\bar{n}_{1}\right)=H\left(\left(\bar{n} \bar{n}_{2}\right)^{k}\right)+H\left(\bar{n}_{1}\right) \quad\left(n \in N_{2}\right)
$$

where $k=\varkappa\left(\bar{n}_{1}\right)^{-1} \in K_{1}$. Fix a compact set $U$ in $\bar{N}_{2}$ such that $\bar{n}_{2}$ stays within $U$. Since $r-d>d$, we can, by Lemma 89, choose, for a given $\varepsilon>0$, a compact set $V_{0}$ in $\bar{N}_{2}$ such that

$$
\int_{c_{V_{0}}} e^{-\varrho(H(\bar{n})}\{1+\varrho(H(\bar{n}))\}^{-r+d} d_{2} \bar{n} \leqslant \varepsilon,
$$

where ${ }^{c} V_{0}$ denotes the complement of $V_{0}$ in $\bar{N}_{2}$. Put $V=V_{0}{ }^{K_{1}} U^{-1}$. Then if $\bar{n} \in{ }^{c} V$, it is clear that $\left(\bar{n} \bar{n}_{2}\right)^{k} \in \in^{c} V_{0}$. Moreover, since $h$ remains bounded, we can choose $c_{1}$ such that $c^{r+1} e^{-\varrho(\log h)} \leqslant c_{1}$. Then we conclude from Corollary 1 above that

$$
\Xi(h n)(1+\sigma(h n))^{-r} \leqslant c_{1} e^{-\varrho(H(\bar{n}))}(1+\varrho(H(\bar{n})))^{-r+d} \quad\left(n \in \bar{N}_{2}\right) .
$$

We may obviously assume that $d_{2} n=d_{2} \vec{n}$ under the mapping $n \rightarrow \bar{n}$. Therefore since $\varrho\left(H\left(\bar{n}_{1}\right)\right) \geqslant 0$ by Lemma 85 , it is clear that

(1) This means that they stay within compact sets as $x$ varies in $\Omega$. 


$$
\int_{c_{V}} \Xi\left(h n_{0} n\right)\left(1+\sigma\left(h n_{0} n\right)\right)^{-r} d_{2} n \leqslant c_{1} \int_{c_{V_{0}}} e^{-\varrho(H(\bar{n}))}\{1+\varrho(H(\bar{n}))\}^{-r+d} d_{2} \bar{n} \leqslant c_{1} \varepsilon
$$

This proves our assertion.

\section{§ 44. Proof of Lemma 21}

We now come to the proof of Lemma 21. Put $A_{1}+=\exp a_{1}{ }^{+}, \mathfrak{a}_{1}+$ being the set of all $H \in \mathfrak{a}_{1}$ where $\alpha(H) \geqslant 0\left(\alpha \in \Sigma_{1}\right)$. Then $M_{1}=K_{1} A_{1}{ }^{+} K_{1}$ and $M=M_{1} A_{2}=K_{1}\left(A_{1}{ }^{+} A_{2}\right) K_{1}$. Therefore it is obviously enough to consider the case when $m=h=h_{1} h_{2}\left(h_{1} \in A_{1}{ }^{+}, h_{2} \in A_{2}\right)$. Put $r_{1}=r^{\prime}$ and $r_{2}=d+r-r^{\prime}$. Then it follows from Lemma 89 and Corollary 1 of Lemma 90 that

$$
e^{\varrho_{2}(\log h)} \int_{N_{2}} \Xi(h n)(1+\sigma(h n))^{-(r+2 d)} d_{2} n \leqslant c_{1} e^{-\varrho_{1}(\log h)}(1+\sigma(h))^{-r},
$$

where $c_{1}$ is a positive number independent of $h$. Since

$$
e^{\left.{\underline{O_{1}}}_{1} \log h\right)} \Xi_{1}(h)=e^{\varrho_{1}\left(\log h_{1}\right)} \Xi_{1}\left(h_{1}\right) \geqslant 1
$$

from [4 (j), Lemma 36], the first statement of Lemma 21 is now obvious. The second is an immediate consequence of Lemma 90 and the relation $M=K_{1} A K_{1}$.

\section{\$ 45. Appendix}

We now use the notation of $\S \S 27,28$. Put $\varrho_{i}(H)=\frac{1}{2} \operatorname{tr}(\operatorname{ad} H)_{\mathfrak{H} i}\left(H \in \mathfrak{a}_{\mathfrak{p}}, i=1,2\right)$ so that $\varrho=\varrho_{1}+\varrho_{2}$. Let $M_{1}$ and $A_{2}$ be the analytic subgroups of $G$ corresponding to $\mathrm{m}_{1}=$ $\mathfrak{i}+\mathfrak{p} \cap[\mathfrak{m}, \mathfrak{m}]$ and $\mathfrak{a}_{2}=\mathfrak{l}$ respectively. Then $M=M_{1} A_{2}$ and $d(m a)=e^{\varrho(\log a)}\left(m \in M_{1}, a \in A_{2}\right)$. Hence it follows without difficulty that

$$
d^{-1} X \circ d=X^{\prime} \quad(X \in \mathfrak{m})
$$

where $X \rightarrow X^{\prime}$ is the isomorphism of $\mathfrak{m}$ into $\mathfrak{M}$ given by $H^{\prime}=H+\varrho(H), \quad Y^{\prime}=Y \quad(H \in \mathfrak{l}$, $\left.Y \in \mathfrak{M}_{1}\right)$. This gives rise to an automorphism $v \rightarrow v^{\prime}$ of $\mathfrak{M}$ which preserves $\mathbb{Z}_{1}$.

Now let $H \in \mathfrak{a}_{\mathfrak{p}}+$. Then

$$
d(\exp H) \Xi(\exp H)=e^{\varrho_{\mathbf{s}}(H)} \Xi(H)=e^{-\varrho_{1}(H)} e^{\varrho(H)} \Xi(H) .
$$

The assertion of Lemma 47 now follows immediately if we apply [4 (j), Theorem 3] and observe [4 (j), Lemma 36] that

$$
1 \leqslant e^{\varrho_{1}(H)} \Xi_{1}(\exp H)
$$


Since $\mathfrak{g}=\theta\left(\mathfrak{n}_{2}\right)+\mathfrak{m}+\mathfrak{n}_{2}$, it is clear that

$$
\left(\mathfrak{S}=\theta\left(\mathfrak{N}_{2}\right) \mathfrak{M N}_{2},\right.
$$

where $\mathfrak{R}_{2}=\subseteq\left(\mathfrak{n}_{2 c}\right)$. We know (see the proof of the corollary of Lemma 13 of $[4(q)]$ ) that

$$
z-\mu_{0}(z)^{\prime} \in\left(S_{1} \mathfrak{n}_{2} \quad(z \in 囚)\right.
$$

Put $u=z-\mu_{0}(z)^{\prime}$. Then $u$ commutes with $\mathfrak{l}$ and since

$$
\mathfrak{G}=\mathfrak{M N R}_{\mathbf{2}}+\theta\left(\mathfrak{n}_{2}\right) \mathfrak{S},
$$

it is obvious that $u \in \theta\left(\mathfrak{n}_{2}\right)\left(S \mathfrak{H}_{2}\right.$. This is the result needed in $\S 28$.

Now suppose $g$ and $G$ are defined as in $\S 7$. Let $A$ be a Cartan subgroup of $G, A_{0}$ the center of $A$ and $\tilde{A}$ the normalizer of $A$ in $G$. Put $W_{A}=\tilde{A} / A_{0}$. Then $A_{0}$ is open in $A$ and $W_{A}$ is a finite group (see $[4(0), \S 20]$ ). We denote by $x \rightarrow x^{*}$ the natural projection of $G$ on $G^{*}=G / A_{0}$.

Let $\mathfrak{h}$ be the Lie algebra of $A$ and $d x$, $d a$ the Haar measures on $G$ and $A$ respectively. Put $G_{A}=\left(A^{\prime}\right)^{G}$ as usual (see $[4(0), \S 20]$ ).

LEMMA 91. Let $d x^{*}$ be the invariant measure on $G^{*}$ such that

$$
\int_{G} f(x) d x=\int_{G^{*}} d x^{*} \int_{A_{0}} f(x a) d a \quad\left(f \in C_{c}(G)\right) .
$$

Then

$$
\int_{G_{A}} f(x) d x=\left[W_{A}\right]^{-1} \int_{A} \nu(a) d a \int_{G^{*}} f\left(a^{x^{*}}\right) d x^{*} \quad\left(f \in C_{c}\left(G_{A}\right)\right)
$$

in the notation of $[4(0), \S 22]$, where

$$
v(a)=\left|\operatorname{det}\left(\operatorname{Ad}\left(a^{-1}\right)-1\right)_{\mathbb{B} / 4}\right| \text {. }
$$

Let $\phi$ denote the mapping $\left(x^{*}, a\right) \rightarrow a^{* *}$ of $G^{*} \times A^{\prime}$ onto $G_{A}$. Then we know (see [4 (o), $\S 20])$ that $\phi$ is regular and $\phi^{-1}(x)\left(x \in G_{A}\right)$ contains exactly $\left[W_{A}\right]$ points in $G^{*} \times A^{\prime}$. Hence our result follows from a simple computation which gives the functional determinant of this mapping.

\section{References}

[1]. Borel, A. \& Harish-Chandra, Arithmetic subgroups of algebraic groups. Ann. of Math., 75 (1962), 485-535.

[2]. Bruhat, F., Sur les représentations induites des groupes de Lie. Bull. Soc. Math. France, 84 (1956), 97-205.

[3]. Gindikin, S. G. \& Karpelevič, F. I., Plancherel measure of Riemannian symmetric spaces of nonpositive curvature. Soviet Math., 3 (1962), 962-965. 
[4]. Harish-Chandra, (a) Representations of a semisimple Lie group on a Banach space, I. Trans. Amer. Math. Soc., 75 (1953), 185-243.

(b) Representations of semisimple Lie groups, III. Trans. Amer. Math. Soc., 76 (1954), 234-253.

(c) Representations of semisimple Lie groups, V. Amer. J. Math., 78 (1956), 1-41.

(d) Representations of semisimple Lie groups, VI. Amer. J. Math., 78 (1956), $564-628$.

(e) The characters of semisimple Lie groups. Trans. Amer. Math. Soc.., 83 (1956), 98-163.

(f) Differential operators on a semisimple Lie algebra. Amer. J. Math., 79 (1957), 87-120.

(g) Fourier transforms on a semisimple Lie algebra, I. Amer. J. Math., 79 (1957), 193-257.

(h) Fourier transforms on a semisimple Lie algebra, II. Amer. J. Math., 79 (1957), 653-686.

(i) A formula for semisimple Lie groups. Amer. J. Math., 79 (1957), 733-760.

(j) Spherical functions on a semisimple Lie group, I. Amer. J. Math., 80 (1958), 241-310.

(k) Spherical functions on a semisimple Lie group, II. Amer. J. Math., 80 (1958), $553-613$.

(l) Invariant eigendistributions on semisimple Lie groups. Bull. Amer. Math. Soc., 69 (1963), 117-123.

(m) Invariant distributions on Lie algebras. Amer. J. Math., 86 (1964), 271-309.

(n) Some results on an invariant integral on a semisimple Lie algebra. Ann. of Math., 80 (1964), 551-593.

(o) Invariant eigendistributions on a semisimple Lie group. Trans. Amer. Math. Soc., 119 (1965), 457-508.

(p) Discrete series for semisimple Lie groups, I. Acta Math., 113 (1965), 241-318.

(q) Two theorems on semisimple Lie groups. Ann. of Math., 83 (1966), 74-128.

[5]. Helgason, S., (a) Differential geometry and symmetric spaces. Academic Press, Now York, 1962.

(b) Fundamental solutions of invariant differential operators on symmetric spaces. Amer. J. Math., 86 (1964), 565-601.

[6]. Langlands, R. P., The dimension of spaces of automorphic forms. Amer. J. Math., 85 (1963), 99-125.

[7]. Mackey, G. W., Infinite-dimensional group representations. Bull. Amer. Math. Soc., 69 (1963), 628-686.

[8]. Segal, I. E., Hypermaximality of certain operators on Lie groups. Proc. Amer. Math. Soc., 3 (1952), 13-15.

[9]. Selbera, A., Harmonic analysis and discontinuous groups in weakly symmetric Riemannian spaces with applications to Dirichlet series. J. Indian Math. Soc., 20 (1956), 47-87.

[10]. WEYL, H., Theorie der Darstellung kontinuierlicher halbeinfacher Gruppen durch lineare Transformationen, III. Math. Z., 24 (1926), 377-395.

Received October 22, 1965 